THE D. H. HILL LIBD,ARY NORTH CROLINA STATE COLLEGE

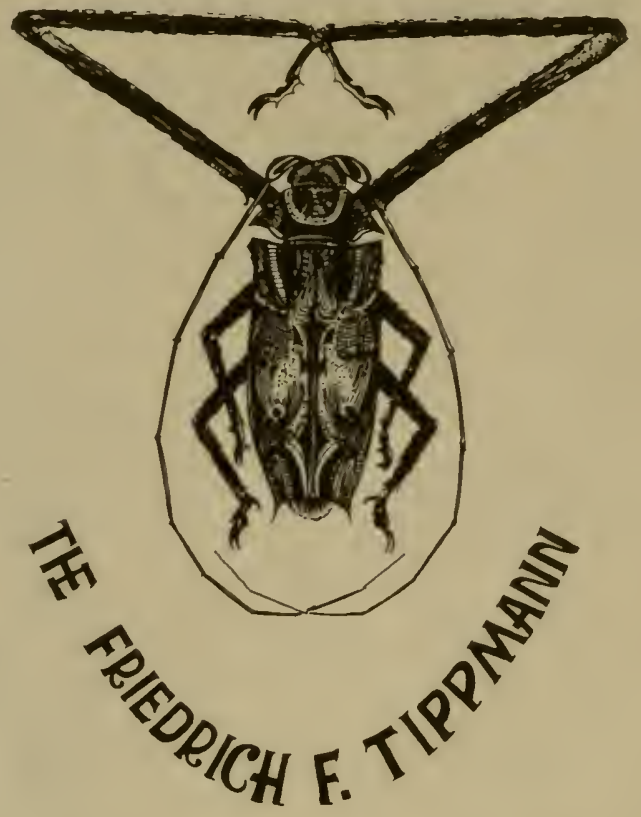

ENTOMOLOGICAL COLLECTION 


\section{8}

This book may be kept out TWO WEEKS ONLY, and is subject to a fine of FIVE CENTS a day thereafter. It is due on the day indicated below:

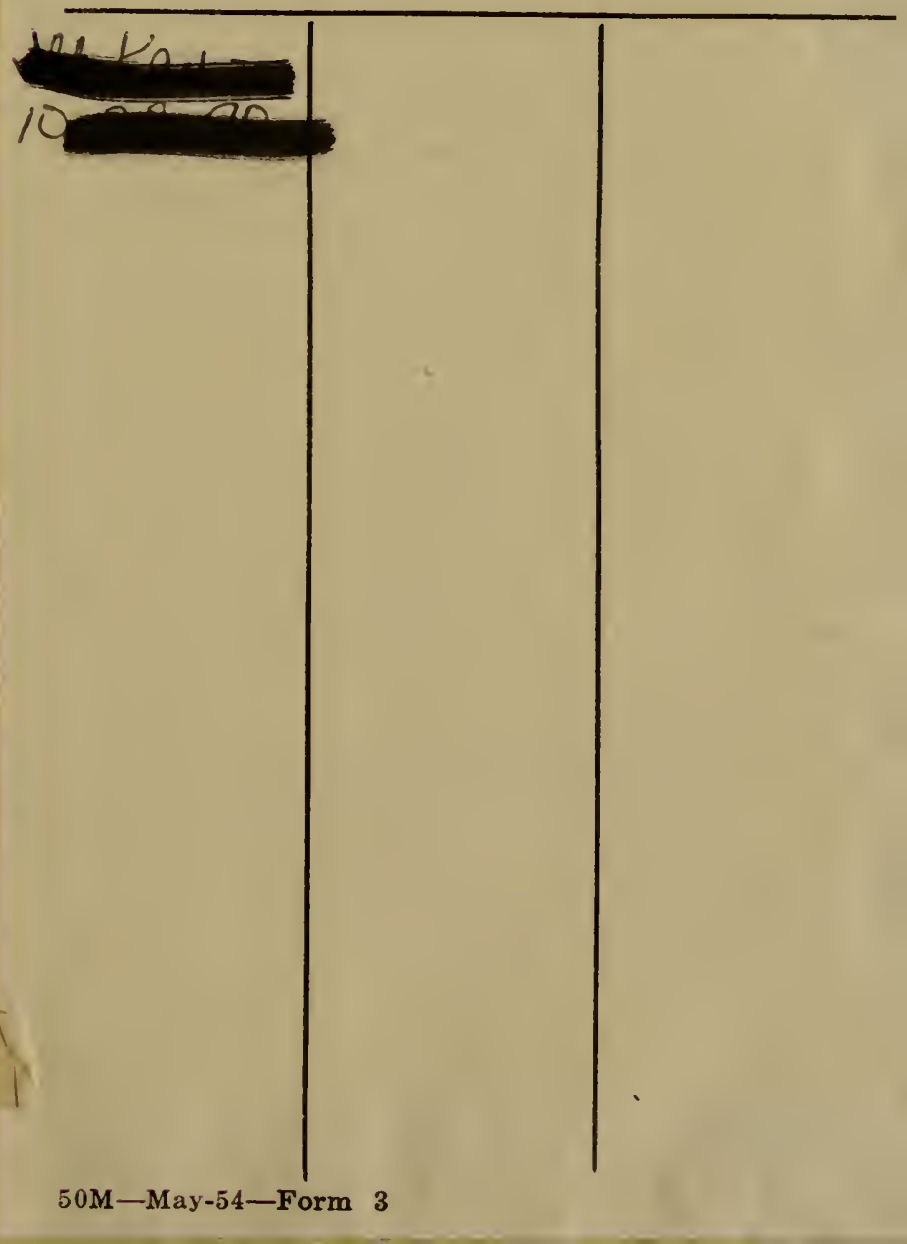





\title{
(j) $\mathfrak{x} \| \mathfrak{d} \mathfrak{v} \mathfrak{i}$
}

\section{Der

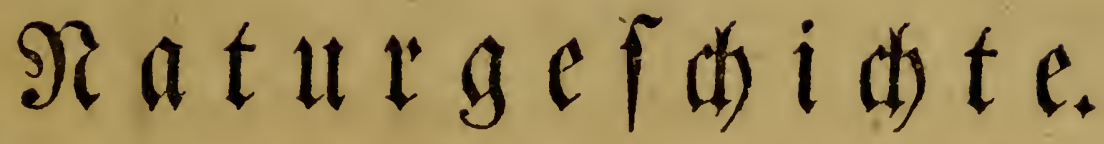

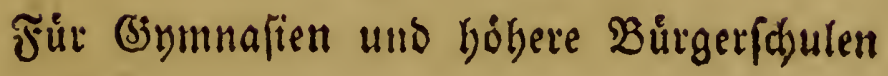

\author{
entworfen
}

บon

Dr. Şerm. V̧ur $\mathfrak{r}$ eifter,

Regree Det Raturgefdidte ain Siolnifhen Real:Ģymnaffum und Privatoogenten an Der Univerfitit zu Berlin.

Dritte verbelferte $\mathfrak{a}$ ufrage.

$$
\begin{aligned}
& \text { Berlin, } \\
& b \text { e (5. } \Re \text { e } i \text { m } \text { r. } \\
& 1836 .
\end{aligned}
$$




\section{r \\ Mี i 6 แ 4 แ' 98}

119

3)

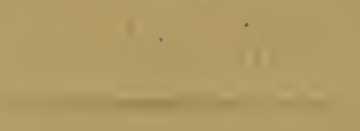

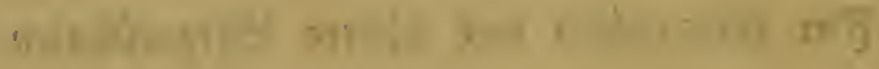

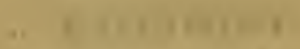

$-$

$$
\text { is } 1
$$

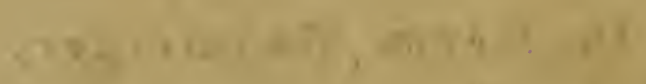

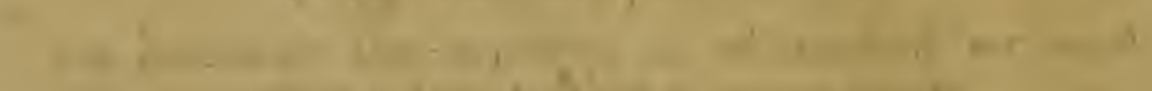

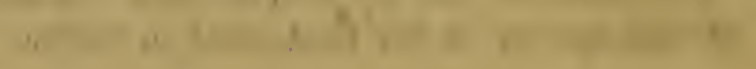

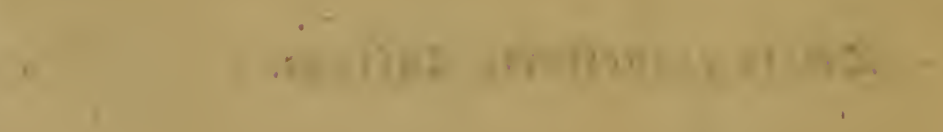

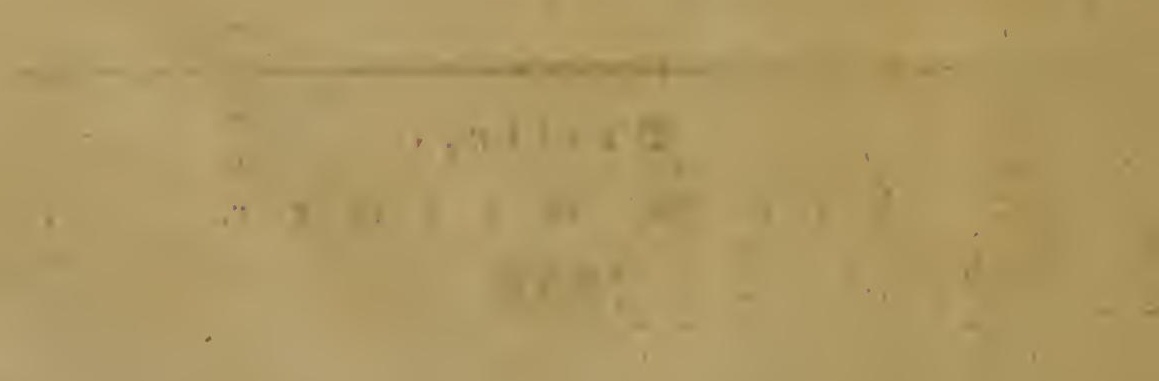




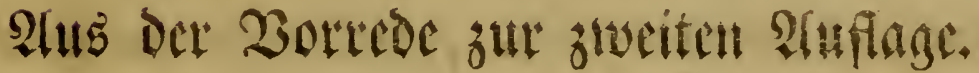

Den fabuellen 2rofag ber erften 2ruffage meines (brundriffes (fite erfdien im Sflober 1833) babe idf fủr einen gůltigen Beweiß feiner Braudgharét, il Der ifm cinmal gegebenen (Seftalt, getyalten, und on ber bei biefer neuen 2fusgabe nux bas geandoert, was

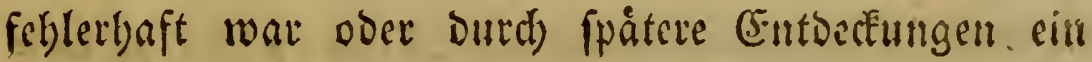
veråndertez 2nnfegn erlitt. So dirften denn die weinngleid) für ben Humfang bes (Jönzen mur getingen Derionderungen bod) wejentlid) ju feiner Servefierung beigetragen baben. -

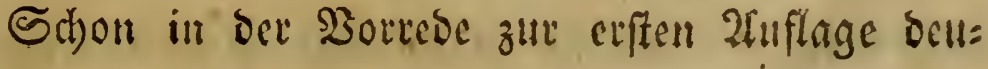

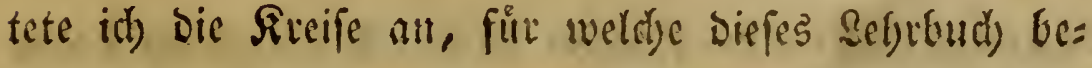

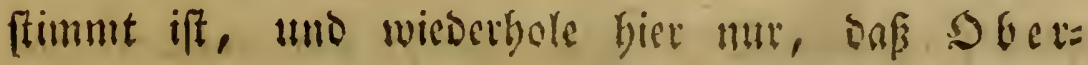


quatta und bie beiden coetus bon sertia, ober wenn, wie bei einigen (jommajien, aud) $S_{e}$ Eunda in zwei Soetus getbeilt ift, nocb beffer beibe Tertia unb Unter= SeEunda die Flaffen find, in weldse bie Siaturgefdidbte, oer 2luffaffung Des vor= liegenden Refrbudbes- nad.), paffend verwiefen rerben múpte. Nan woilde bei folder 2Unlage bes Unter= rid)tes in ber unterften Flaffe am zweckmáßigften mit Der 300 logie Geginnen, und in Dem einen Şalb= jabr bie Růtegrattbiere, in dem anderen bie übrigen (5tuppen burdhnebmen. Darauf folgte it bit nách= flen Glaffe bie Botani£, aud jåbrig, im Winter Die Serminologie ober der allgemeine Ibeil, im Som= mer ber fpeziclle, verbunben mit Excurfionen. F̌̉ Die britte Klaffe bliebe bann bie Mineralogie, wel= dbe fich recht gut in einem balben Sabre vortragent låpt, wobei del seflet nod) 3eit genug bebålt, die Sxyftallographie ctroas weitlänfiger, als roie fie bier gegeferi wuite, ourdjunelmen, etwa in ocm Umfange, wie idf fie in meinem Sondruch-oer Raturgefdidfe (berlin 1350. 3.) gegetien bate,

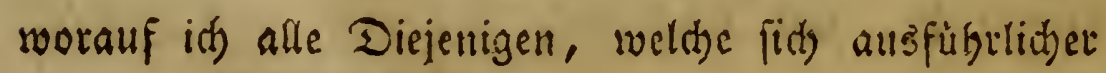


úber nur angedeutete (Begenftánte untertid)ten wollen,

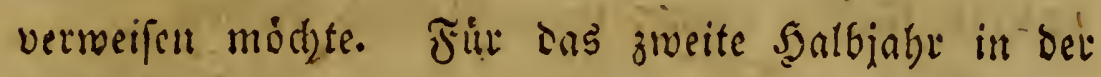
Dritten Flaffe dúrfte ein bunbiget Surfus bet (5) ed: logie, weldben fich freilid) ber Sebret nad den yol: Gambenen Suulfsmitteln, unter benen fith be $\mathfrak{l}_{\mathfrak{a}} \mathfrak{b} \mathfrak{e}$ be (3) eognofie, überiegt von S. v. Ded)en (Zertin 1832. 8.) als bas brauchbarfe auszeidnct, felbft entwerfen mufte, am geeignetfen frin; doch Eounte er alud) mit einem repetitorifthen Bortrage bet früber burdsentommenen Discipliten ausgefullt werden; be: Fonders geborte bieber bann die Iarfellung des $n a=$ tưliden Eyftems ber Pllanzen, für weldje ebenfalls mein Sandoud ber Paturgefdidte als $21 n=$ leitung dienen formte. Derjelbe (biang bes llntertid): tez leibet úbrigens nut) auf Birgerfdulen 'feine 2rn: wentung; auch für bicfe witroe id eine Reifyenfolge set Disciplinen in bet angegebenen Dronnng als bie zwedmåkigfte vorfhlagen. (5s bat autb) in Der Dar: ftellung des (siegenftandes eime foldhe Stufenfolge des Unterrid)tes mir vorgefducbt, woraus fidi benn auch eine megr wiffenfdaftlidje șaltung fur bie allgemei:

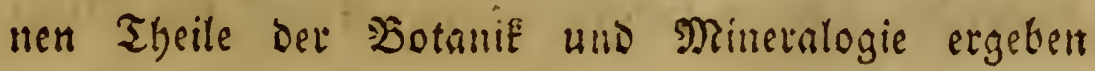


muste; legtere namentlid) durfte einem Sobuler ber unteren- Slaffen immer unberftändic) Gleiben.

Da in ber Raturgefdichte alles auf 2 fufdomung allommt, fo berfåme ber Sebrer ja nicht, dic be: rủbrten Segenftånbe ben Schulern, fo viel es fid) thun lápt, felbft vorzulegen. Spráparate werden wobl die wenigften (Sdulen befifen; aud) reidjen gute 2tbbil= sungen bin, ja find in vielen Faallen, befonders bei nieberen Thieren, vorzuzieben; ba fie fdon die d)arat= teriftifden Meremable ganz befonders bervorbeben. Leiber feblt es noch immer an einer zroedémápigen Sammlung fưr den Bedarf eines Sdúlers, daber id) fhon in Der Borrede zur erften 2Uuflage cinen zoolo:

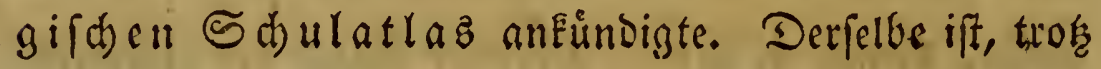
nandber Sillderniffe, fo weit gesieben, dap bas erfte

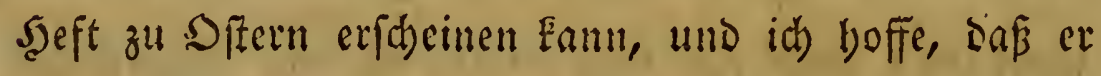
fich ourd Sraudbarkeit und Woblfeilbeit, wie siefer

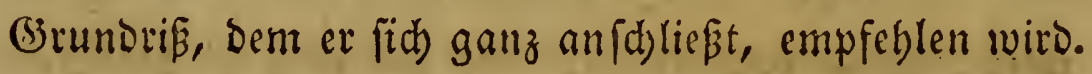
Ger iff übrigens fo eingeridjtet, Daß er felbit bem Stu= Direnden nod) Mittel jur 2CHfflårung bietet, mitbin Den Bedürniffen cines (S)nmnafialvortrages vollémmen entpridat. Beim Stubium Der Sotanif fino 2rbbils 
Eungen weniget notbig. Seidjt fann ber Selgret die cigentbunlityen Formen, weltye in ber Ierminologie

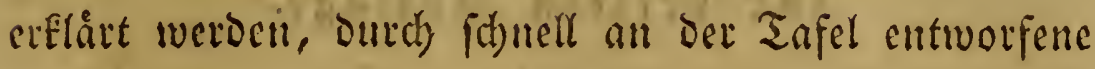
Umriffe eilåutern, und bicje 2frt ber Derdeutlidung

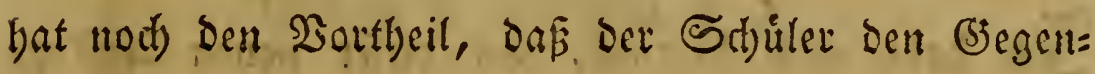
ftand gleid)fam vor feinen 2rugen entfteben fiebt. Sa babe diefe Metbode nidbt blop in Der Botanie, fon=

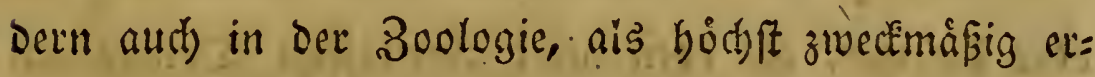
fannt, aud) barnuf gebalten, baß bie Sóbler alle von mite vorgezeidgneten Figuren fogleid in einem be: fonbern Sefte copiren uno wågreno ber Repetition als Fingerzeige benusen. Freilid geborrt bazu cine fid)ete Şand und treue PGantafie; allein obne diefe Fanu cin Siaturforider iberbaupt nithts anfangen, und baber barf ids fie, alz nothrwendige (sigenidaften jebes Saturbiftorifers, wobl bei den Rebretn ber Pgys fisgraphie vorausfergen. 


\section{$23 \cup \mathfrak{P C D}$ \\ zur oritten $\mathfrak{F u f l a g e . ~}$}

Fin babe nidb bemúft, bei biejer neuen 2suflage nur foldse Berbeffertungett anzubringen, weldse burd) Den Fortfdritt ber Wiffenfdaft notfwendig rurben, ober Die mir, als eben nicht úberflúffige 'Detailanga: ben, befonders fúr bie genauere Unterfferibung von Woidhtigêeit forienen; alles Hebrige ift geblieben. Der in Der Borreve zut zreiten 2ruflage angebentete 2ft: las ift inzwifhen fálon erfobienen, wenigftens. 3 şefte Deffelten; ex Eann von Der Berlagshandung, fo wie aud) burd) alle úbrigen Buchbandlungen Deutid)= lands, bezogen werden. Scine 2fusfúfrung wito ifn 2rlen am beften empfeblen.

Berlin im 2 luguft 1836. 


\section{E $\mathfrak{i} \mathfrak{n} \mathfrak{l} \mathfrak{e} \mathfrak{i} \mathfrak{t} \mathfrak{u} \mathfrak{n} \mathfrak{g}$.}

§. 1. Die $\mathfrak{R}$ aturgefdichte hanbelt von ben Formen,

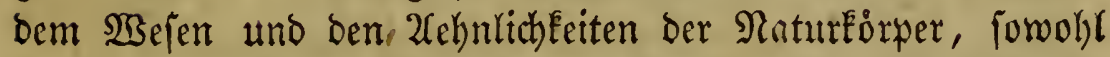
bem auseren ats auch bem inneren $B$ nute nach. Sie bejd)reibt biefelben uno ftellt fie, ihrer \$erwandtichaft gemáz, in gróßere uno Eleinere. Sirippen ( Rlaffen, Sronungen, Familien, (3at= tungen uno 2(rten) - ju[ammen.

§. 2. Die Raturfirper jerfallen ifrer Beftalt und in= neren (Eimridhtung nach in zivei grope (S)upwen.

Die einen befteben auterertich wie innertich aus veridsies benen aheilen, beren jeber eine gewiffe sanz beftimmte Set= rid)tung hat, wermitterft melcher biefe Raturfirper fich erhals

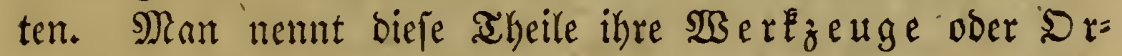
gane, und bic Saturforper barnach organif d e.

Die anderen beftehen entweder gar nicht aus verfochiebes nen İheilen, oder aber wenn fie alb verfdiesenen Beftand= theilen beftehen, fo hat boch keiner berferben eime serrichtung

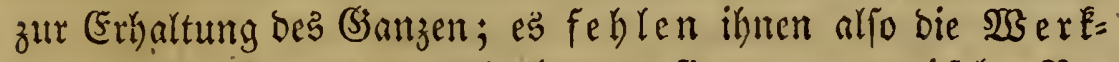
zeuge ober Srgane, weshalb man fie anorganifhe $\mathfrak{N a}=$ turforper genaunt hat. Soldyer 2 frt fino alle Mineralien, bie Steine, Metalle u. bgl. m.

§. 3. Die organiften :aturforper theilen fith wieder. in zwei grope (Gruppen, nehmlith:

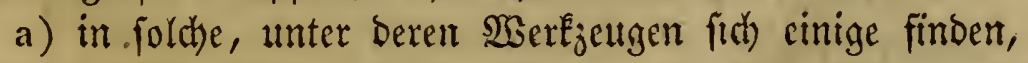
welche ben Raturtortper ganj ober jutm afheil in $\mathbf{B e w e g u n g}$

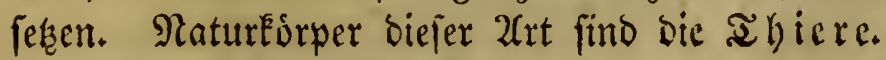

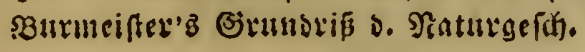


b) in jolthe, eenen bie WBerfyeuge futr Bewegung, mit= bin auch bie Fábigkeit fich bewegen zu fonmen, mangetn; bieje nennt man $\mathfrak{D}$ flanzen.

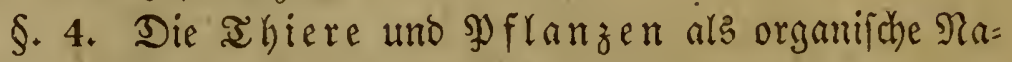
turferwer nennt man auch lebendig, und falsecibt alfo bei= Den $\mathfrak{e} \in \mathfrak{b} \mathfrak{e n}$ zut.

23as ift aber bas $\mathfrak{E} e$ ben?

Das acben ift $\mathfrak{x}$ hatigkeit aus eigener Siraft, eine Ihaitigkeit alfo, bie Eeimen Sirtmo von auken erbilt, fondern bie fich jelbit antreibt, bie leoiglich inrer felbe weigen thátig ift.

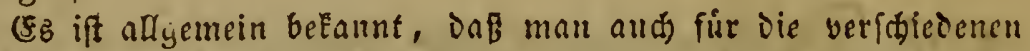
Beránderungen Der leblufen giaterie gerwiffe firáfte als Urfaden bc: tractetet, und daf wir ble Sirdfte, welche Die Merúnderung oder Die Thátigkeit Derferben úberbaupt veranlab̧ten, nathmeifen Eönnen als ausgegangen von anieren Daterien oder bervorgerufen Durdi Deren Sirafte. Sein leblofer Sirper theilt fith von fetbft, fondern erfit in Jolge einer von aufen einroilkenden Siraft, Bein leblofer Ssörper be:

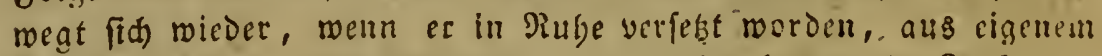
2lutriebe; Eeiner rubet, fo lange er nod eine bemegende Siraft bat, menn iGn nidft eine andere siraft feftbält. - Tlber die lebend: gen Siorper thun Dies allez alls freiem 2ntriebe; Die prange faugt

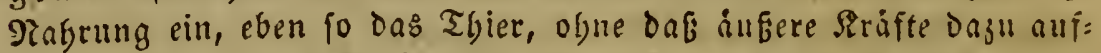
foriern; jene treibt Sabuflinge, 3reige aus freiem 2ntriebe, Diffe

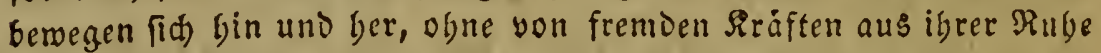
gefturt worden gu fein u. f. w. Disfes fteie szandeln alfo ift icr wefentlide Unteridied der lebendigen und leblofen fiórper.

§. 5. Nach ber angegebenen \$erichiedenbeit ber Natur=

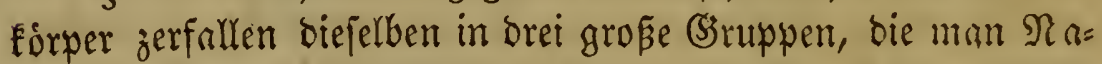
turreiche nennt, und eben fo theilt fict) bie Naturgerchichte in brei grope 2rbtheilungen, welche fint:

a) Die Naturgeichichte ber 'ahtere, oder bie Soologie, welche vom agherreich handelt.

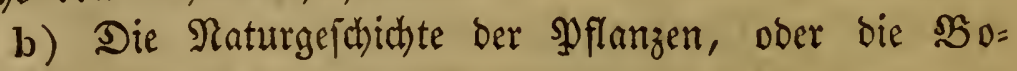
tanik.

c) Die Naturgefchichte ber anorganijchen, leblofen $\mathfrak{N a}$ turfórper, ober bie Mineralogie. 


\section{Erfer $\mathfrak{A} b$ fditt. \\ $3 \mathfrak{D} \mathfrak{o} \mathfrak{l} \mathfrak{v} \mathfrak{g} \mathfrak{i} \mathfrak{e}$.}

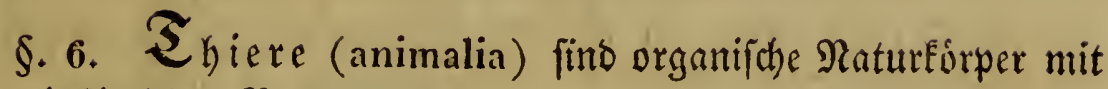

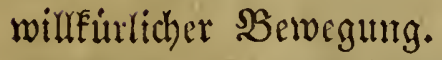

Unter einct willférliden Bewegung verffeben wir cine folde

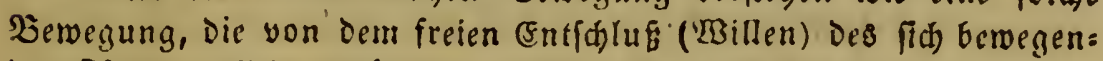
Den fistpers allein abbángt, uno Reiner anderen ?necgung von auben bedarf. Das Dermigen, Den Ort, wo fith Der RaturEirper befindet,

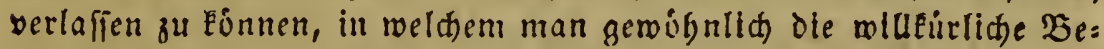
megung überbaupt ausgebríct glaubt, eommt nidt allen Thieren zu, es feblt Den feftgerbadjenen goolnpen, Diufdeln uno manden parafiten.

§. 7. Die Thicre, als organiiche Maturkórper, befteben aus mekreren Siganen ober $\mathfrak{W S e r k}_{j}$ eugen, welche zufammen ifren $\mathfrak{e}$ eib bilben.

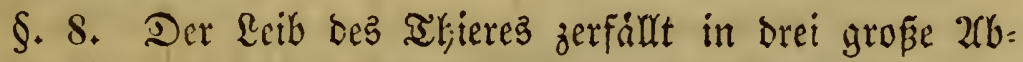
fdituitte, nehnilid) in ben $\mathfrak{l}$ opf (caput), ben $\mathfrak{R}$ umpf (trun-

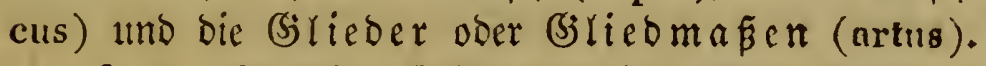

§. 9. Der Sopf Fommt nicht allen ahieren ju, ná mentlicl) fehlt er den Mufdeln, den Seefternen, Me= oufen, Jolypen uno Snfufionzthieren, die man bes= harb auch forfloje a aliere (animalia acephala) nennen kinnte.

\$. 10. Der $\Re$ umpf ift allen ahieren ohne 2 fubnahme eigen; cinige baben nidbts weiter als ben Sumpf, bieje mennt man wohl $\mathfrak{B a u d h}$ thiere (animalia gastrodea oder ga-

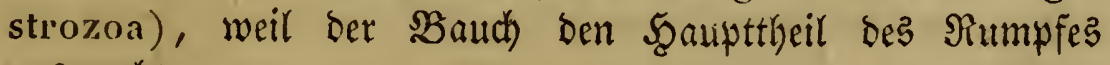
ausmacht.

§. 11. Die (5jlieber feblen ebuftalls vicèn arbieren,

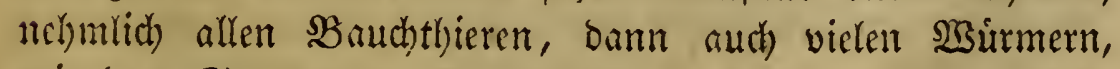
wie bem Spulwurn, Siegenwurm u. a. m.

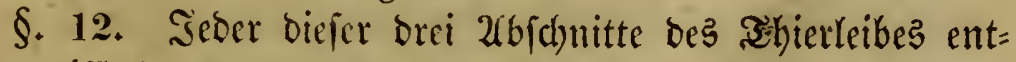
hâlt gewiffe Srgate, welche nur an oder in ifm vorfommen.

\section{H. HILL LIBRARY1*}

North Carolina Siate College 


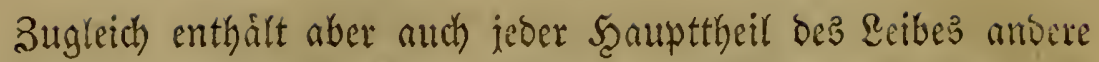
Srgane, bie fich in allen oreien wieberfinden, biefe fint:

a) Die Merven (neuri), weise Fisten, bie fich wie bie. 3weige cines Baumes von einem SJauptitamm aus verbrei= ten und mit ihren álEerften (Enden zu allen alloeren Sorga= nen jich hinbegeben. SGre Serrichtung ift' bie (5mp fin= bung, welche baher aud huberall moglich ift. Sie ents ipringen entrlich alfe aus dem (3) ebirn (cerebrum), bas im Sopf liegt, und als bie şurgel Der Nerben angepelen weroen fann.

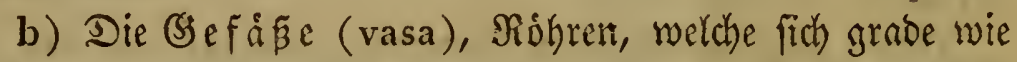
bie Rerven zmeigformig werbreiten und ju alen anoeren $D_{P}=$ ganen begeber. Shre Serrichtung befteht Darin, Den Rarl)=

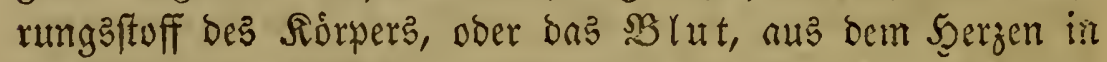
alke Srgane zu fübren, Domit biefe aus ihm ihre Mathrumg fhopfen, und ben uibrig gebliebenen Seft wieder zum Seerzea

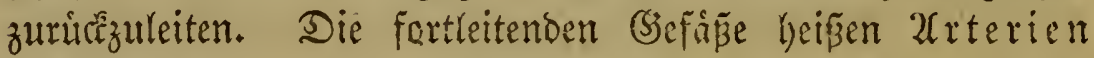

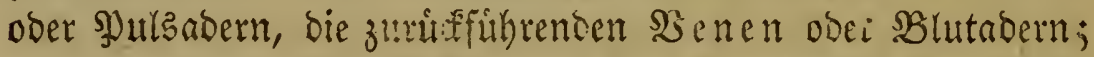
erftere verrathen fich leitht burch ifire \$Bewegung oder \$)ulz: fchlag, baber fie auch Schlagabern genannt werben.

c) Die $\mathfrak{M}$ ủkeln (musculi), bidé, 'rumbe, oder flache, breite, geftreifte Srgane, bie aus lauter feinen Fafern beftec her, weldhe alle warallet nteben cinnomer, ober aud wohl in melyreren fich Ereuzenden Schichten itber einander liegen. Shyre Serridtung befteht Darin, bie Sigane, an melchen fie fith bes finven, in Bemegung zu jetzen, baker fie ats bie cigenttichen Bewegungsorgane ber åhiere zu betrachten fint.

Swifhen ben Mluskeln liegt cine aus vielen Şajen uno

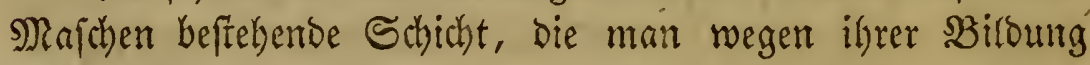
3eltgewebe nennt, und in weldyer fith bas Fett anjam=

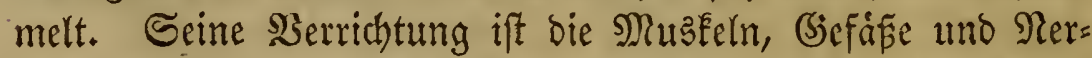
ven cinzulyullen, bamit fie von aupen geichulazt fint. SBeide, Mubketn und Zellgewebe mit bem Fett, bilden bas Fleifch Der athiere.

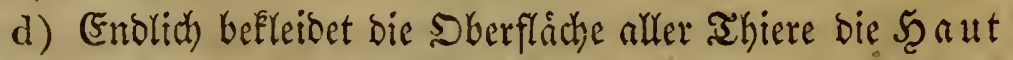
(cutis), welche als eine ichitgende Scitlle zu betrachten ift, un= 
ter ber bie Srgane, wie.unter einem enganjabliesendoen Schleier, verftectit liegen.

§. 13. Die Sorgane, welthe fich nur in Den einzelnen Scauptabjchnitten bes Reibes befinden, laffen fich am beften nach biefen Scauptabtheilungen betrachten.

§. 14. Der Fopf enthalt bie Sinneswertzeuge,

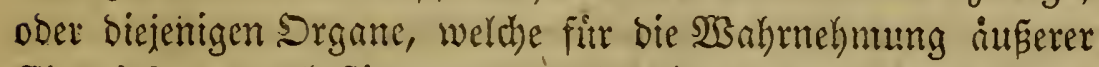
Findricte ganj beftimnter 2fit berechnet find. Esz giebt be= ren vier.

a) Das $\mathcal{A}$ u ge (oculus), ober bas Sorgan, vermittelit mel= d)es wir feljen. (Fj liegt immer an ber vorderen Seite bes Sopfes, utub ift in ben meiften Fâten boppelt, fehr felten ein=

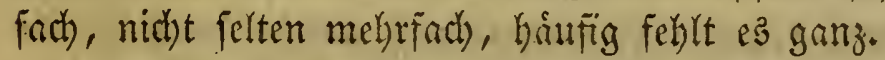

b) Das $\ \mathfrak{h r}$ (auris), oder bas Drgan, vermittelft mel= dhes wir ben Schall und bie $\mathfrak{x}$ ine, welche andere Rorper von fich geben, wahrnelymen, riegt immer an ber Seite des Ropfez, uno ift ftets, wo es fich auch finden mag, boppelt; febr vie= len ahieren fehlt das Sorgan bes (jeborrs vollentmen, andere horen, obwohl bas \$hr noch nicht mit \$Beftimntheit bei ifnen entoceft morber.

c) Die Nafe (nasus) als (J)eruchsorgan nimmt bie Eindricie walle, welche gewiffe flitedtige ober veroantpfende Materien erregen; fie findet fich immer am Sorbertheile bez Sopfes unter ten 2fugen, uno befteht in ber Regel aus ei=: ner Doppelten Scokle, in welcher fich cime zarte mit Merwen verfehene Scaut verbreitet. Sehr viclen ahferen fehlt auch bie Rafe ganj.

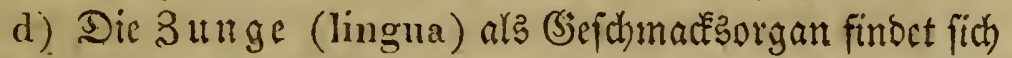
im Muthe unto beftelyt aus einent fleijhigen, auth wohl von

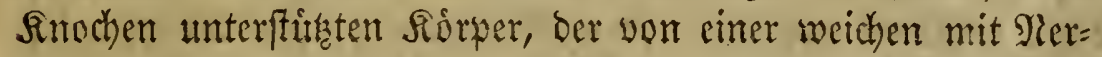
wen verfehenen Scaut itberjogen ift. Das a

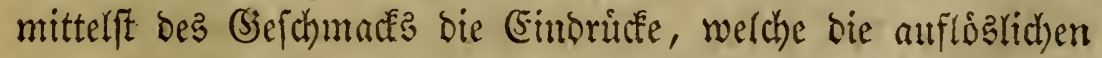
Sorper alf bie Nerwen Der Sunge macthen, balber nicht alle Dinge ichnedflear fint. Die, Sumge ift allgemeiner verturcitet als bie Slafe, aber nicht bei allen abieren, bie eine sunge biben. Dient fie zum Sdymedent. 


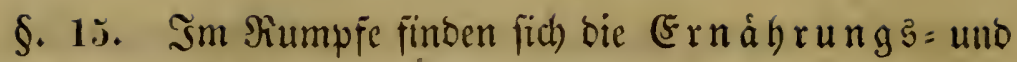
Fortpflanjungsorgane ber abiere.

I. Die Ernabrungsorgane baben ben 3medf, die= jenigen Stoffe, weiche bas Thlier ju feiner Erhaltung beonrf, ihm jujufithren uno juzuberciten. Dicjen 3weck erreichen fie

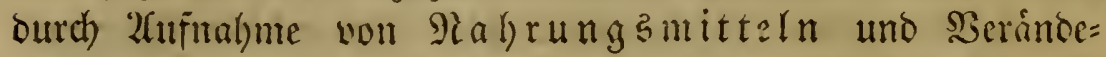
rung berfelben in bie ernifhrende Subftanj ober bas $\mathfrak{B} \mathfrak{I} u t$.

Xufgenommen werben bie Nafyrungsmittel burd) ben Muno (os), ber allen Thferen cigen ift, in ben $\mathfrak{R}$ agen

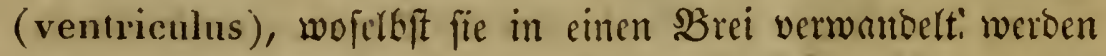
uno nun in Detr Darm hubergeben. Wus bem Darm fau=

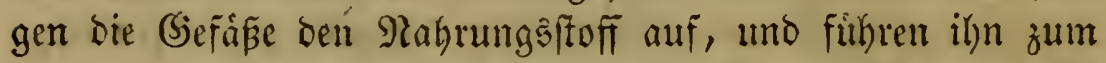
Şerzen (cor), einem musfuilofen Sade, weldher burch Schei= bewánde in fanmmern uno \$orbofe getheilt miro, bie mit ein= ander in SSerbinoung ftehen. Son bier aus foum er in bie $\mathfrak{L}$ unge (pulmo), banit er burch) bice, vermittelfit ber ein= geathmeten $\mathbb{R} u f t$, eine Sierinderutig (Sicinigung) erleibe, bie ihn zur (Ernábrumg oer Srgane geichiff́t macht. Siun ift ber $\mathfrak{R a b}=$ rungsfftoff wal)res $\mathfrak{B} \mathfrak{l}$ ut (sanguis), bas baun entweder jum Şerjen juriuffelhrt umb aus ithm in alle Theile bes Siorper?. vermittefft ber 2 rterien oder ) ulzabern geleitet wiro, oder

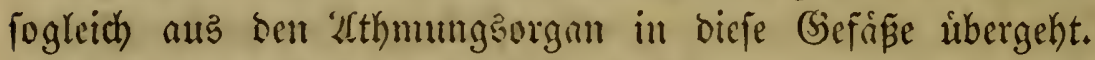
Der aheil bes \$Blutes, welden die Srgane ibrig gelaflen ha= ben, Kehrt mit bem meu aus bem Darm aufgefogenen Saft.

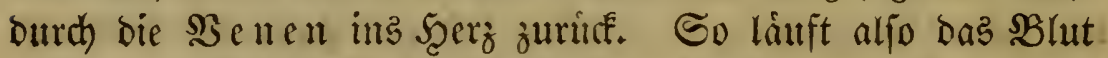
auf einer Sreistann im Siorper umber, welche \$ewegung beshalb $\mathfrak{A}$ reisla

§. 16. Die Dignate, welche wir fo chen fenmen gelernt baben, liegen fo im Siumpf, bas bie \&unge ben oberften

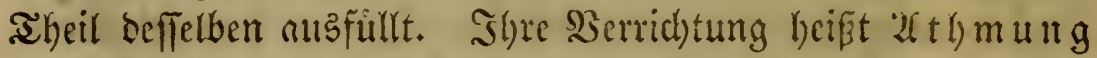

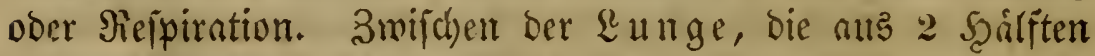
befteht, liegt bas Scerj. Siefer ganje Roum, welchen beide einnelymen, heist Brufterfen (thorax); bei ben Sauge=.

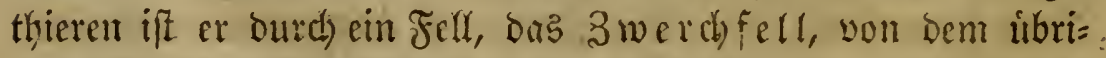
gen afyeil Des Siumpfes getremut. (Silcich unter bem smerch=

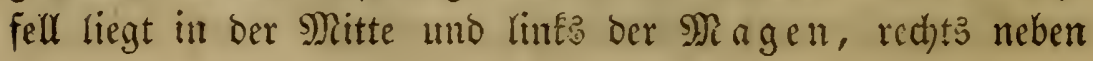


it)m bie $\mathfrak{a} \mathfrak{e b e r}$ (hepar), von welther bie (3) alle (bilis) ab= gejonbert wiro, cine grimlichgerbe bittere Frlinfigkeit, bie in ben

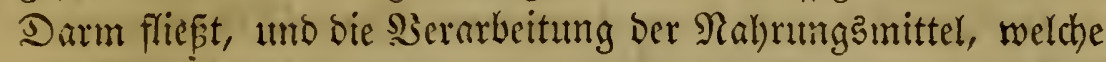
wir \$̧erbau ung (digestio) nennen, mit bewirken bilft. Der

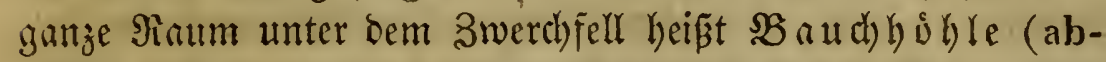
domen ober venter), uno enthâlt mutr nod) ben $\mathcal{D}$ arm, ber wieber auts bem Diunbarm (ilium) und Diff barm. (colon) befteht, welcher lefectere fich in ben 2ffter muindet. 2uth finden fich in biefer Scible noch bie Nieren (renes), zwei

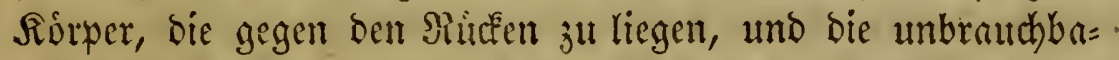

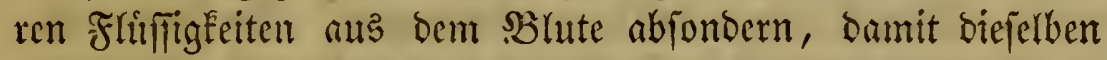
als Sבarn ober Urin wieder abfließen fonnen. -

§. 17. II. Die శoortpflanzungzorgane find Samale ober Sicfe, in welchen Frlinfigfeiten abgefondert werben: Beim Wsibchen bilden fich in ihnen bie (Fier (ova), aus benen bie

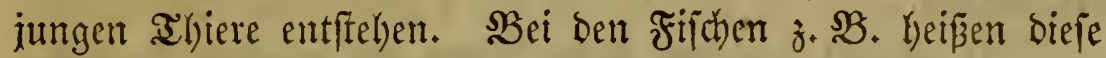
Sacte dee Meibchens Rogen, des Mánnthens Milch. Die allermeiften Ibiere entftehen nus (Fiern, weldhe bie Siscibchen legen; einige, wie bie Sångethiere, gebăren lebenbige Sungen;, bie von Der Nutter emókrt, gefíugt, werben. -

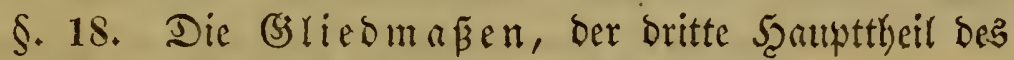

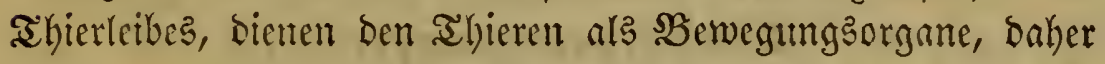

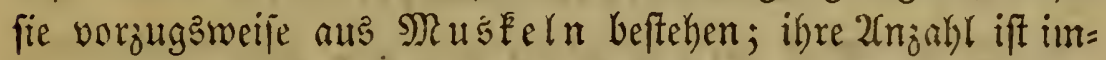
mer eine $\mathfrak{g}^{\mathrm{r}} \mathrm{ab}$, auch fitzen fie ftets cinander gegenitbor, fom= metrifh an beiben Seiten bes \&eibes, und haben panrweis

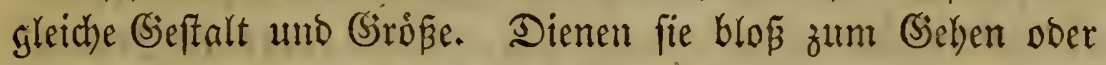

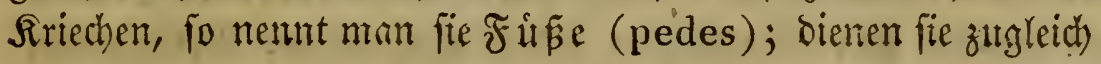
zum Ergreifen ober Fefthalten, fo nemt man fie genteinig= lich $\mathfrak{A}$ rme (brachia). Dienen fie blós zum Ergreifen uno Fefthalten Der Siahrumg, fo heisen fie Riefer (mandibulae ober maxillae). Die Riefer ftehen in Der Rabe bes sundez

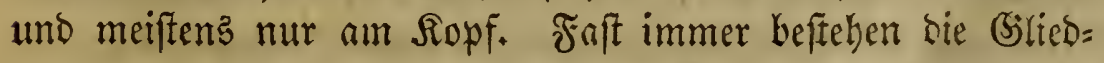
mẫen ats binter einander liegenden Gjliebern, bie jich ein= zeln gegen einander bemegen fónnen. .

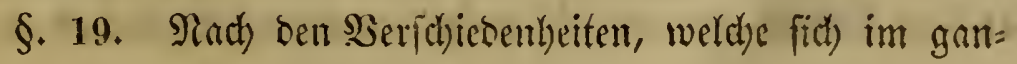


Erfter 2rbfonitt. Boologie.

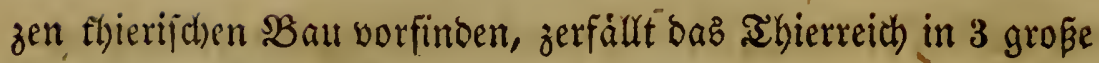
Siruppen. Diefe find:

1. Die $\mathfrak{B}$ a uth th iere, Gastrozoa. Shr $\mathfrak{e}$ eib iff ein bloper

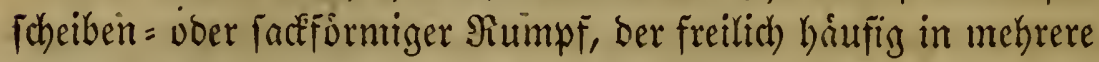
Strablen auslduft, aber niemals mit wabren (Glieomazen verie: Gen ift. 'Manche von ihnen baben cinen אopf', sie imeiften nic'st.

2. Die Grieberthiere, Arthrozoa. Shr \&eib befteht aus vielen, hinter einamber liegenden, gleichen ober ungleichen Ringen. Die meiften baben einen Sopf und wabre (S)led: maß̧en, anderen fehlen beide.

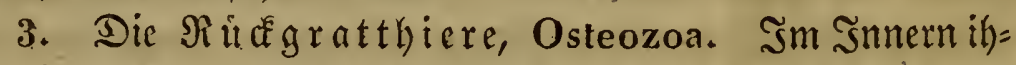
reß Sorpers findet fich ein (Berifft harter Fiorper, bie man Sinochen nemnt, uno an welchem Gierift doer. Skelet alle ibbrigen alyeile befeftigt find. W(Ke Gaben einen beutlichen Sippf, und bie allerneiften vier mehr oder weniger ausgebildete (Stliebmafen.

W3ir wollen mit ber SBetrachtung ber leşteren (Sruppe, weir fie bie befannteften und grioften aldiere enthalt, ben $2 \mathfrak{n}=$ fang machen.

\section{Erfte Scauptgruppe. \\ $\mathfrak{R} \dot{\mathfrak{u}} \mathfrak{d} \mathfrak{B} \mathfrak{i} \mathfrak{a} \mathbf{t} \mathfrak{t} \mathfrak{b}$ i e $\mathfrak{r}$ e.}

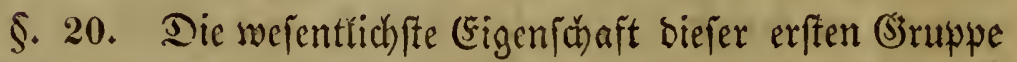

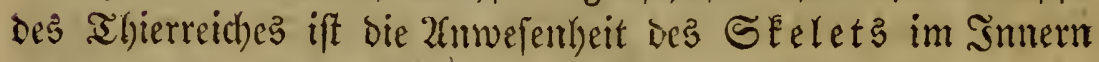
ber bierber gehorigen abiere, Daber wir mit ber Betrachtung deffelben beginnen.

Das Skelet befteht aus Sinochen, bie in ben Gselen=

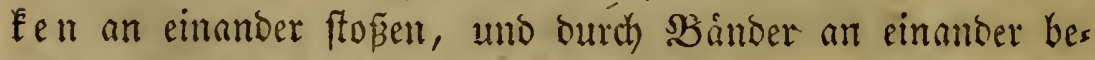
feftigt find. STeder $\mathfrak{A}$ nochen (or) ift eigentlid) ein jelliger Sorper, in beffen Bethen fich phosplyorjaure Salferde abgefert hat. Siele baben im snnern einen hohlen Raum, ber mit einer fettigen Materie, Dem Mark (medulla), angefillut ift. 2hn ben Enden, ba wo dic gegen einander beweglichen Ino= 
d)en. fich berühren, find biefelben mit eimer weid)en, faferigen, weis̄en Subftunz itberzogen; , beren Sberflache fehr glatt iff, uno bie ben Slamen $\mathfrak{A}$ no r pel (cartilago) fingrt; manche Sino= d)en finto auch burch folche Subftan

§. 21. Das Sfelet jerfillt, wie ber gaitje 2 eib, in brei

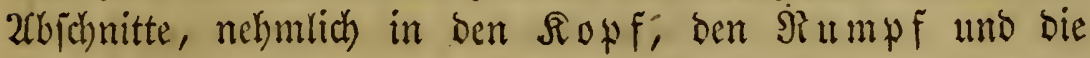
(3) rieoma вen.

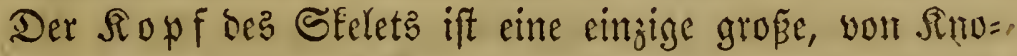

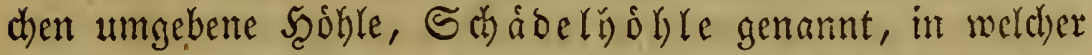
oas (jichirn liegt, uno an welcher bie bubrigen Sinod)en befe= ftigt fint. Sie bilden andere Şublen, bie fid an vorbern Iheil des Schabels befinden uno bie Simmesorgane in fict) aufuebmen; es find: bie 2(ugenhoblen, in weld)en bie

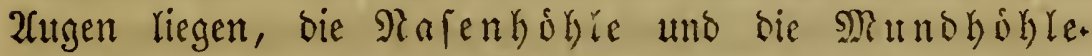
Diefe Yefgtere umgeben bie Siefer, jwei hufeifenformige fino= dhen, beren jeber alls 2 Scilften befteht, bie vorn an cinmoer

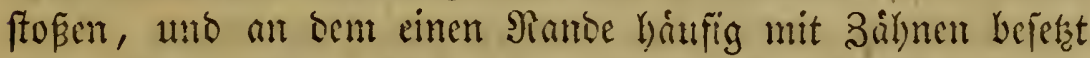
fint. Sn ber Siegel Eann nur ber Unterfiefer bewegt mer= ben; dit aber auch beibe.

§. 22. Der $\mathfrak{R} u m p$ f bes Seletets befteht nus vielen ein= jelmen Sinochen, bie fich unter 3 sibbrifen bringen laffen,

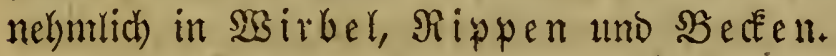

Die $\mathfrak{i s i r b e l}$ (vertebrae) finto fleine aus cinem faft wuirfelformigen Sortper utmo einem von biejem ausgehenden Bogen keftekende Sinochen, bie burch Sinorperjubftam fo an

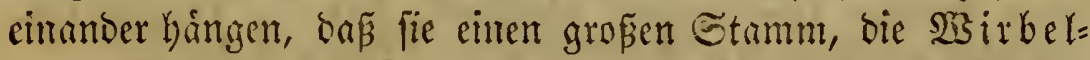

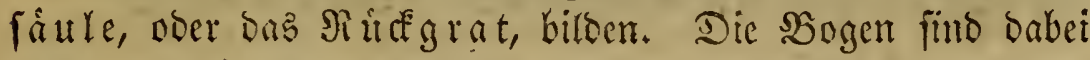

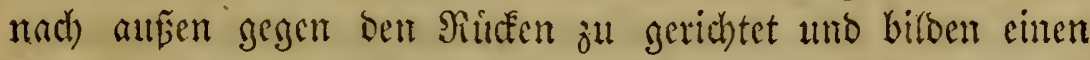

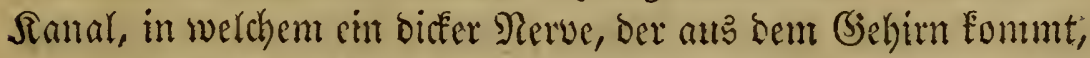

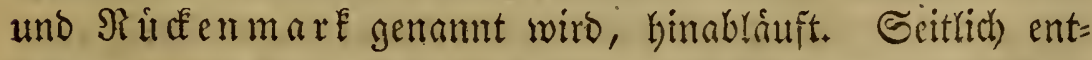

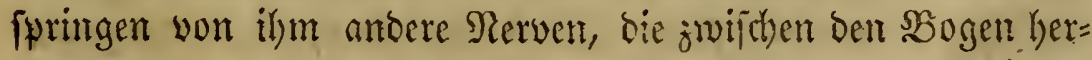

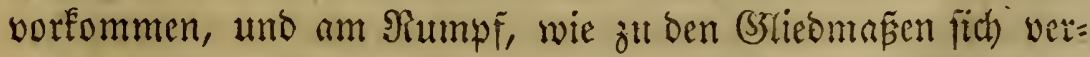
breiten. Som Sogogen entipringen nod) fwişe Snod)enfortfâse, ciner grabe nach außen unt oben, Der Dornfortfas, zwei andere nach ber Seite hin, einer an jerer Ecite, bie $\mathbb{Q u e r}=$

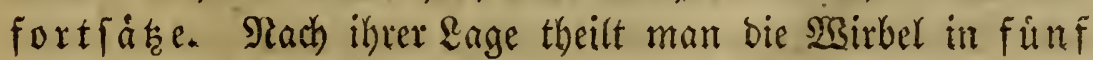


(5ruppen. Şalswirbel beisen bie exften gleich binter bem Siopf, bie Eeine ßippen tragen; nach ihnen folgen bie mit Ripwen verjehenen $\mathfrak{B}$ ruftwirber, bann bie ebenfalls rippen= lofer, aber unter allen am ftáréften gebauten \&endenwir=

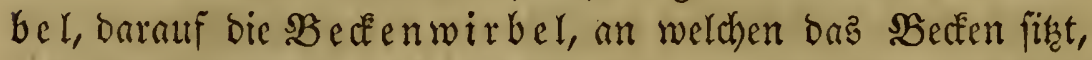
uno die meiftens zut einem Rnochan, bem Şeiligenbein, verwachjen; endict) bie $S_{d y}$ wanzwirbel, bie fleinften ron allen, weldye ben Sdwwanz ber gefdwainzten alfiere bilben.

Die zweite Scruptform ber Snodhen bes Rumpfes find bie Ripyen (coslae), bümne, fhmale, halbfreisformige Jino= chen, welche mit bem einen (Enbe an je zwei Mirbel fto jen, unt bier burch) Binner in einer (Gelenfung befeftigt find; Das

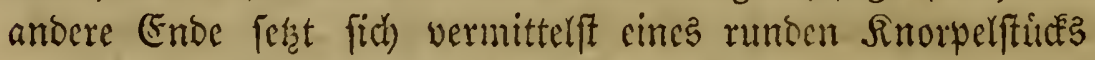
an eimen runben ober flacher, fchmalen Intochen, Der vorn auf ber $B$ ruft liegt, uno baher $\mathfrak{B}$ ruftbein (sternum) heišt. Diefes mit ben Sippen amb Rieffenwirbeln bitbet aljo ben Shruftenften, in weldem Eungen und Scers liegen.

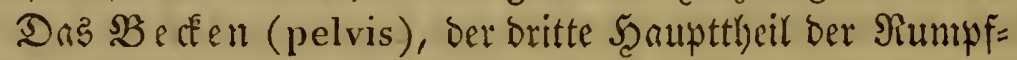
Enochen, bitbet 'bas untere (Ende bes গumpfes, uno befteht aus mefyreren flacken, cine geráumige, aber nur jum sheil

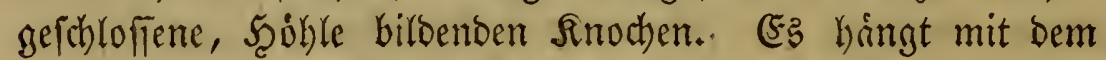
breiten Darmbein jebereits am Sceiligenbein, und bient.

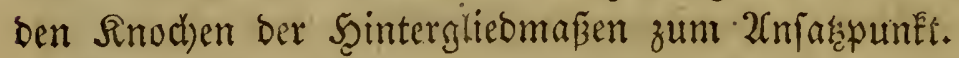

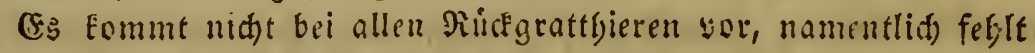
es allen Jifhen, Den Edjlangen, und iff felbje nodj bei Den Sáuge= trjiercn mitunter unvollfommen entwiefelt. 2ulle Diefe Sbicte baben

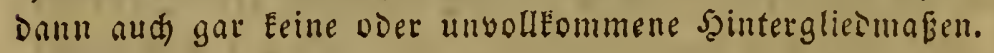

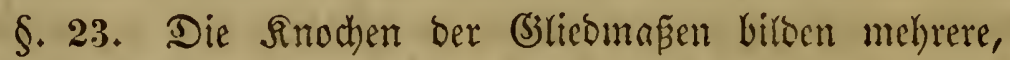
hinter cinander lirgento Neilyen, umo eticheinen als ziemlich. enge unt fefte Riobuen, bie an beiden Enden Keutenformig ver= bickt und aígeruttoet find. Dieje mit Snorpel íberzogenen

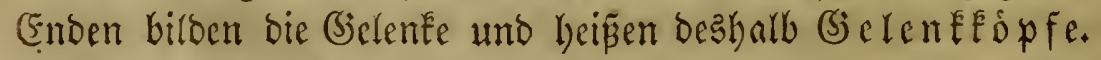

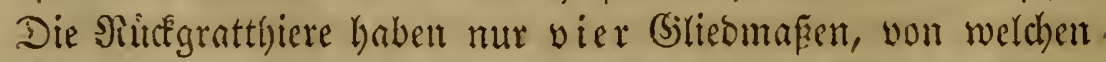
Das erfte \$laar am vorderen, bas zweite in ber Sirget am bin= teren Enbe bes Siumpfes befeftigt ift. Das vorore sjanr, beim Nenfohen $2 \mathrm{rm}$ genannt, baingt an cinem flad)en brei= 


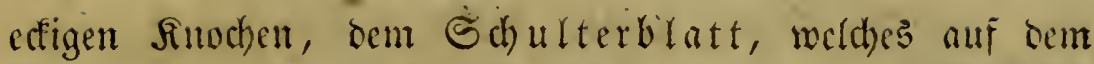

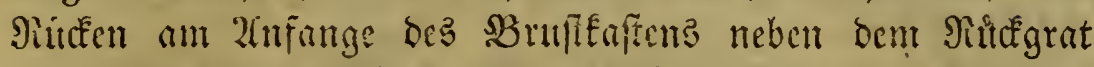
liegt, uno bei benjenigen aldieren, bie ihle vordern (sitieoma=

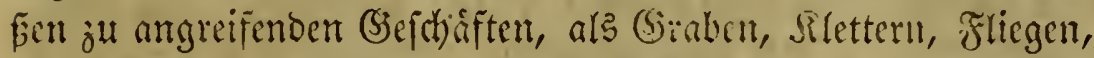
Edjwinmen gebrauchen, burch einen binmen $S=$ formig ge=

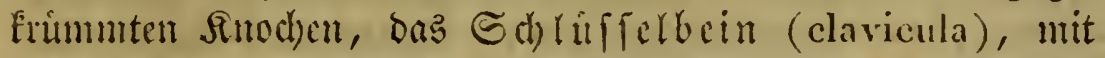

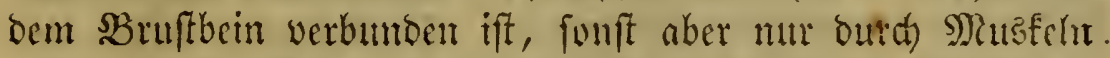
mit bem SBrufteften zujammenhangt. 2in bicfem Echuter= blatt bangt Der grofie $\mathfrak{D}$ berarmeno chen, und an biefent zwei Elcinere, bimtere, bie Speid)e uno (Elle, meldye sin Unterarm bilden. SESO $\$$ berarm uno Unterarm julammenfto=

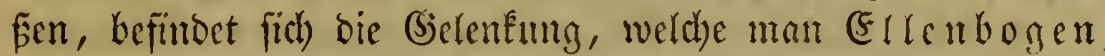
nemt. 2uff bie Sinochen bes Unterarms forgen mebrere (hoich)=

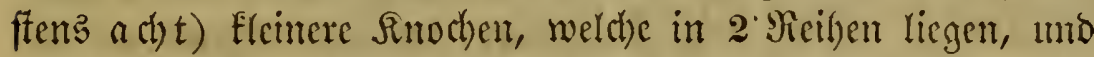
das Scandwurjelgelent bitoen, daher man fie Scand= wurjelfnochen nennt. Utr bieje fto als bie 5zam ober ber Fú 3eken hat, aljo 1, 2, 3, 4 ober 5 ; alle bingen unter fich Dutrd) Mutuleln jufammen, uno bil=

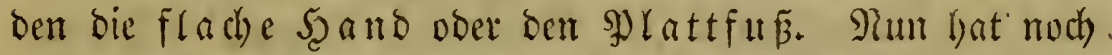
jebe Bebe soer jeder Finger orei finter einander liegente $\mathfrak{A n} 0=$ djen, mit 2(thnalme der grofen Zehe oder des Dau= mens, bem cin bstied weniger eigen ift, als ben übrigen,

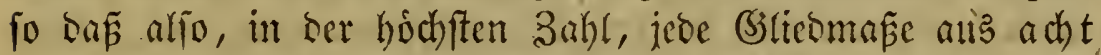
binter einander liegenden Snod)enreihen jufammengejergt ift, mithin eigentlich) 7 (Sielentungen haben mus.

Bei ben Scintergliedmnfen, gewóbnlich Beine ober Scin=

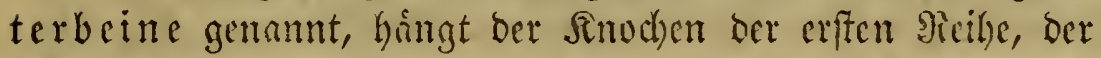
Sberf(b)enerfnoden, im Schiftgelent am Becten; auf

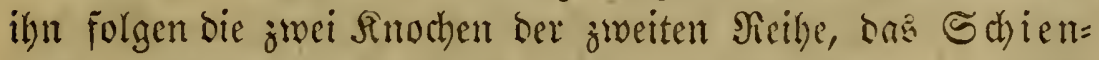
bein und das ฏ feifenbein, weldye im Sniegelent an ifn graingen. 2fuf biefem (solent liegt moch cin rumber Snochen,

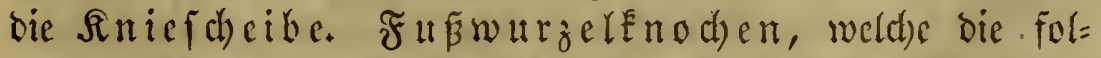

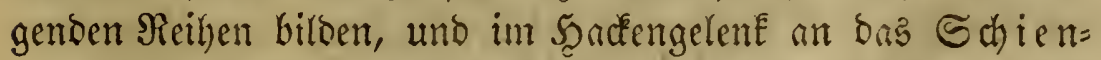

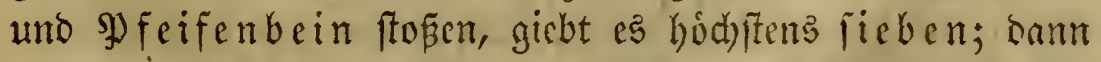
folgen io viele Sinochen, als seben am gur befintich

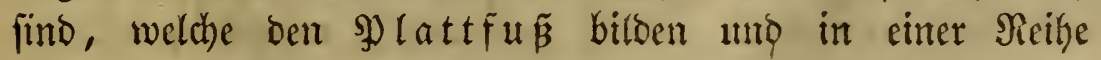


neben cinamber liegen; endlich die 3 Reihen ber 3eken= fnod)

Don Der eben gegebenen befdreibung Der Glieomafen meidtt igr Bau bei vielen Riuckgrattfieren fegr ab. Die Fifhe baben meder Deutlidec Gelente, nod Deutlide getrennte Sinoden in Den cinzeluen Gelcneen, ibre 3eben fino gliederlos uno Durd Şaut gur Flofie ver:

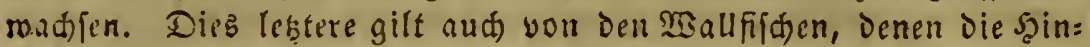
terglicomagen nodj Daju beftándig feglen. Die Sdilangen baben gar feine Brieomafen, uno manden (Fidedffen feblen theits die vorderen, theile die binteren. Bei den ziógeln bilden Dié yorderen Die J̦higel, Die bintercn Die beine. In Diefen finden fich niemals Jufmurzelfng: den, Die PlattfufEnoden fimb in eiren, Den $\mathfrak{E}$ auf (tarsus), ber: wadjen, und biefer figat an ben Gdienbein unmittelbar.

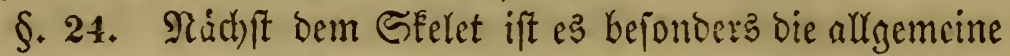
Form, welthe bie Riuctigratthiere von ben ubrigen untericheibet. Slyr Sopf if immer bentlich fichtbar; an ifm nehmen wir in ben allermeiften Fiullen bie angegebenen 4 Sinnezorgane wahr. Bzlinde situfgratthiere finden fich unter ben Fiichen (Dek Sd)leimanl, Myxine), Den 2 mphibien (Caecilia; ber $5 l(m$,

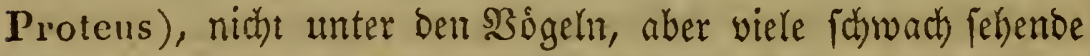
unter ben Sángethieren (ber Maulwurf, Talpa; bie SBlind= maus, Spaląx tr. a. m.). Das SWhr ift nod) fehr verfedt bei Dent Fijd)en; ben 2tmphibien fehlt ber (Gehórgang, Daker bas

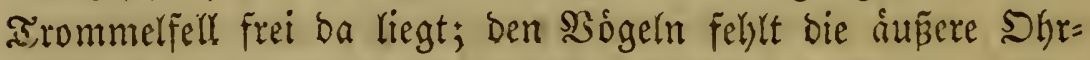
muichet, bie alio nur bei ben Sintgethieten vorkommt. Die

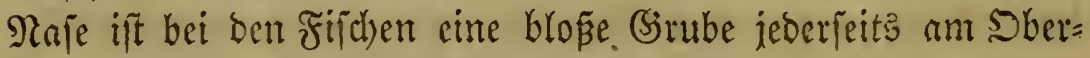
Ficfer, bei den dibrigen eine in Den Mtunt führende Syoble. 2fud) bie Zunge bient wohl nur bei ben Singethieren jum

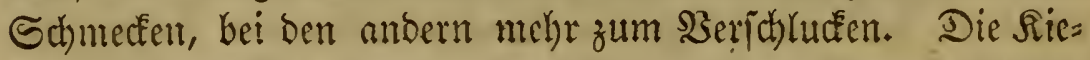

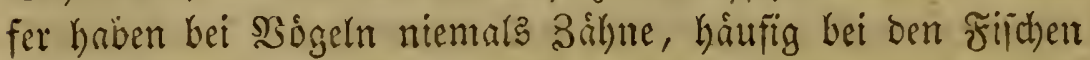
(nidht bei ben Sarnfen) uno 2imphibien (nid)t bei ben Schild = froten), in Der Regel bei ben Sáugetbieren (nitht bei ben ist)=

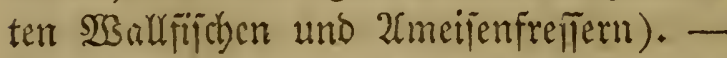

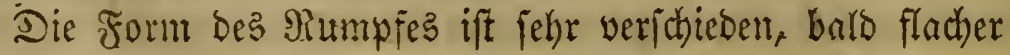
ober ichmaler und boher, wie bei ben Fiijhen; bald lätglidher runber, wie bei ben 2(mphibien; barlo fleiner umo fahnförmig, wie bei ben \$oogeln; endich) am grop̧ten und ftireffen, unt

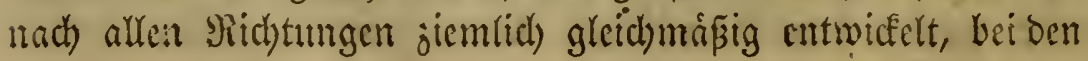


Siugetbieren. Unter fainen inneren Sorganen find bejonters bie jut 2ethmung dienenden am veridtiedenartigften. Die Fiiche

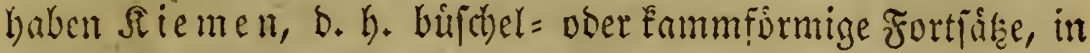

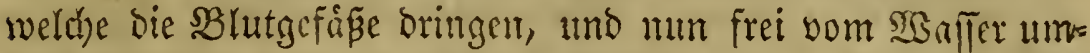

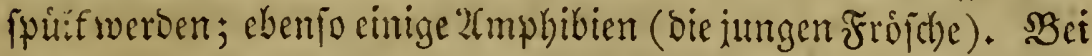

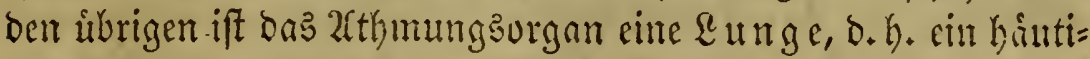
ger Sack, Der inwendig mehrere in Serbintung ftelende Bethen hat, an welchen fich bie Bhutgefáfe verbreiten. Sn biefen Sack

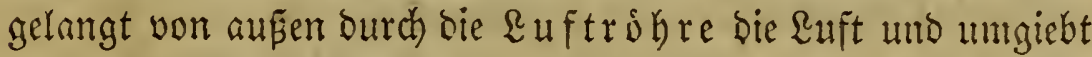

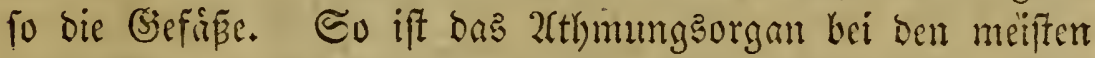

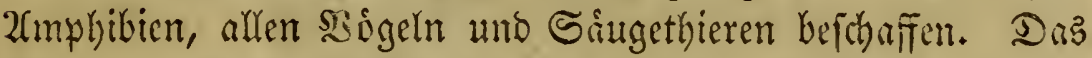

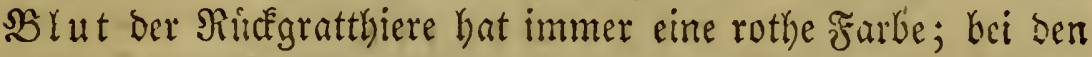

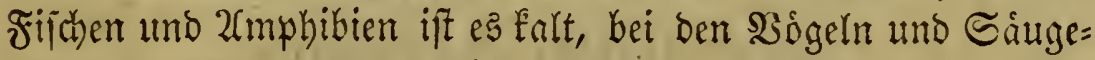
thieren warm, uno hat biet cine Şize von $28-32^{\circ}$. Ser Darmenal jeigt bei ben verfotedenen Ritefgratthieren be= fonbers in ber \&ange grope Serichiedentheit, den Eirrjeften ha =

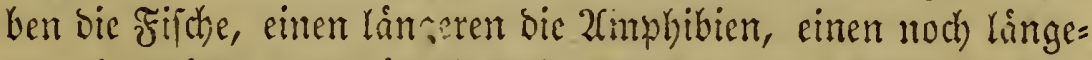
rent bie Siogel, ben langften bie Saitgetbiere. Wuth nach ber

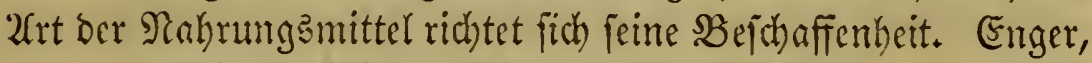
aber fefter, ift er bei ben Fileifldfreffern; leinger, weiter, aber

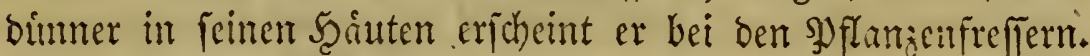
Seine Lange ift oft fehr bedeutend, haufify bas Dreifact)e ber

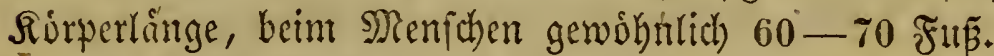

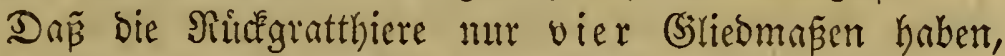
wutbe fchon bemerft; Daf aber biejelben báufitg fehlen und ouch in ilyer Geftalt fehr verichicoen fimo, ift ebenfalls ge= pagt worben.

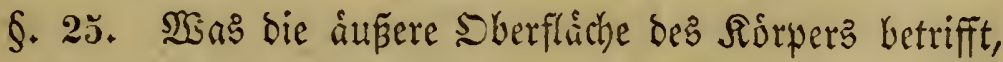
fo ift biefe felten ganz nact, fondern in ber $\Re$ Regel mit baarigen oder fnochernen Gebilben belleidet. Bei ben Fifthen und 2(m= phibien find e's Echuppen ober Echilder, bei ben Sojgeln immer Federn, bei ben Siugetbieren meiftenz Şaare, feltener Schuppen oder Schilder, aber nie Federn.

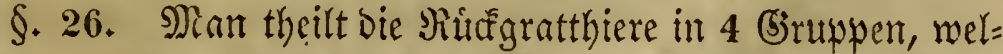
che Slaffen genannt werben, fie fino allgemein befamt und untericheiben fich am leidteften in folgenden Meremablen: 
a) Sällgethiere. Sie haben Bitzen, welche Wild abfondern zur (sinilibung Der Süngen.

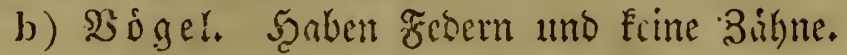

c) 2 mplibien. Sint naft doer haben Schuppen uno Shilder, aber niemals Floffen, fondern entweder féme

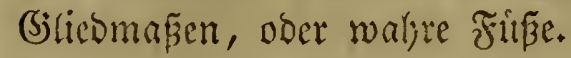

d) Fif he. Scaben Riemen, Schuppen, Schilber ober

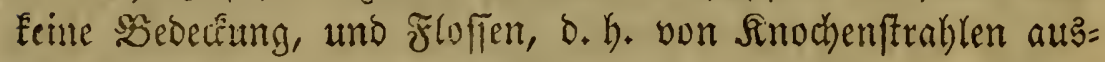
gefpante Şautlappen, als Gslicomaß̄en.

\section{E $\mathfrak{i} \mathfrak{f} \mathfrak{e}$ S $\mathfrak{i}$ a ffe. \\ Siugethiere, Mammalia.}

§. 27. 2Uußer Der bemerften (Figenthumlichéeit unter= icheiden fich bie Saugethiere noch in anderen (Figenfohaften won Den úbrigen Sronungen.

So bat ihr Sopf nur cinen beweglichen Unteritefer, Der obere hängt feft mit bem Schibel zufammen. Die 3ábne fint in ben Jiefer eingebohrt, eingekeilt wic man's nennt,

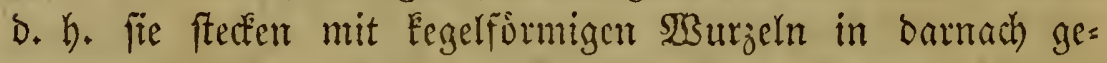
formten (Siruben. Wuch untericheibet man nad) ber Form ber.

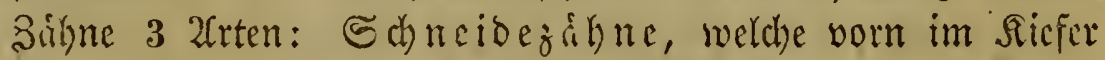
fitzen, uno eine breite, meiselformige sieftalt baben; $\mathfrak{B}$ a d zábne ober $\mathfrak{M}$ abljabne, bie ganz hinten im Siefer fte= dfen, mebr wierectig gebauet find, uno oben eine. breite oder in Backen außlaufente Fliche barbieten; und $(5)=$ ooer 21 to

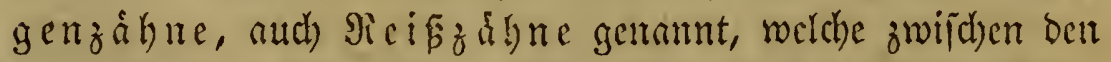

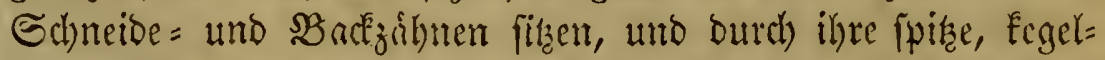
formige (Sieftalt fid) aujzecichnen. Sebe: 3alyn jeigt 2 Scaupt= theile, bie Srone, welche aus bent אiefer hervorragt uno

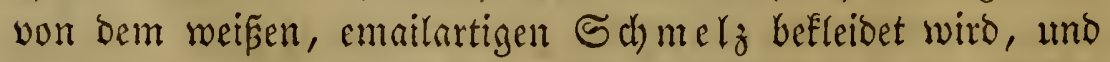

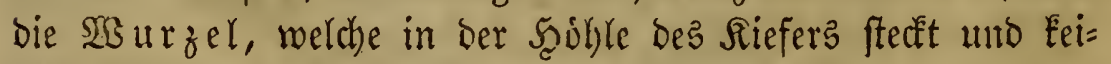
nen Schmelz hat. SBei mandien Badilyen bringt audh ber Schmelz ins Snnere bes 3ahnes cin uno bildet hier Reiften; folde 3álne werben $\{$ ch) melz faltige, bie uibrigen mit Sdhmelz 


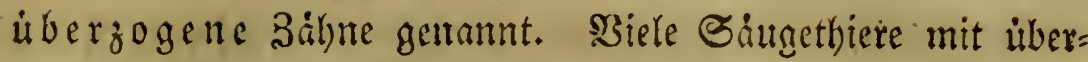
jugenen Bähnen wechjeln bieferben (fbithten) nath einiger Beit; bie mit fchmelzfaltigen Bilgnen gewwhnlich nur bie vorbe=

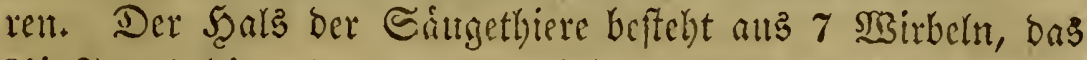

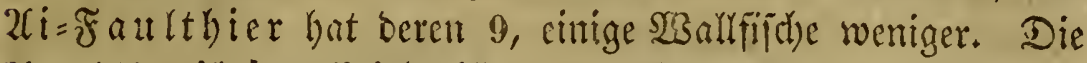

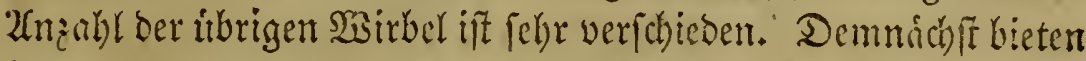
befonbers bie Bahl ber Zehen und ihrer Snudhen Berfchiebenthei= ten bar; eitrjelig ift bas \$JFerd, zweijehig bie Ninber, breigehig bas Nazhorn, vierjelig bas Echwein, füm= zehig bie meiften, $j^{*} \mathfrak{B}$. bie Fleberminule, meiften 2 fifen und

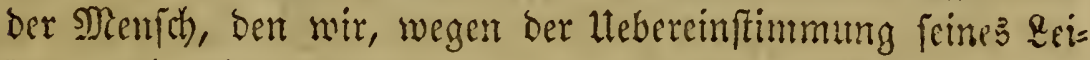
bes, audh mit jut ben Singethieren reci)men. Stebt von ben

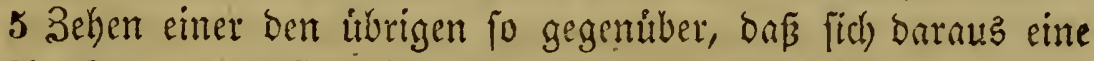
2fit Bange zum Ergreifen uno Fefthalten bildet, fo nennt man

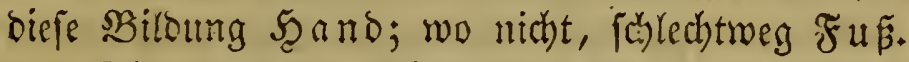

Die Scaut ber Såugetbiere ift felten nadt, mie bei ben WBalfifichen, meiftens mit Scaaren bedecft, nicht feiten aber auth) mit Schildern, wie beim 2rmabill, ober Schuppen, wie beim Schuppenthier. Sebes Scaar if ein horniger Fraben,

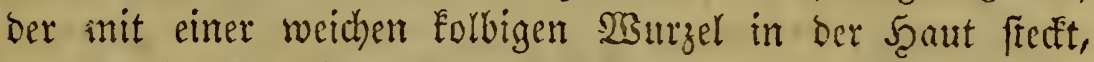
uno burch bicje feine Mabrung einfargt, fo lange bas Jcaar waidyft. SBei einigen Saugethieren wachjen bie Şare beftinte big, bei andern fallen fie im Frubjathr umb Scerbjt aus unt werben von neuen erjetzt; bies nemt man $\mathfrak{r}$ auben. Die Rippen, Naje uno Fusfoblen fino haufig von bicient Scaars kleibe frei., Die Zlugen haben zmei fie bedecfente Szantfal=

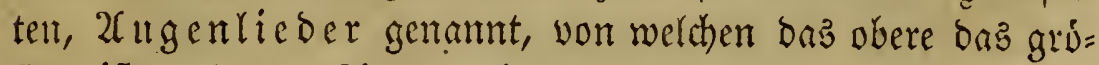
Fere ift. 2(n Den \&ippen jeigt fich meiffens ein Sbart ftákerer

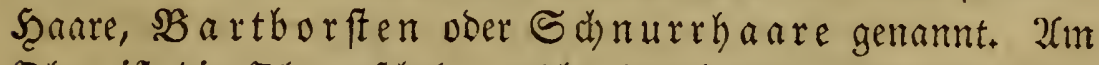

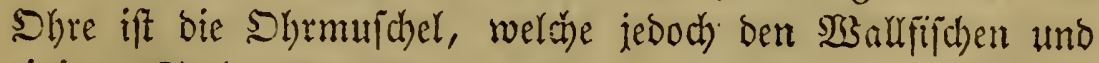
einigen Seehumben fehlt. -

Die Spitze ber Behen ift bei allen Saugethieren mit eincr lornigen şlatte ober Scafen bedeç't, weld)en man $\mathfrak{R} a g e l$ (unguis) nennt. Riegt berierbe flach auf ber obern Seite ber Sebe, fo beipt er sুlattnagel (lamna), biegt er fich itker das Ende fort, fo mennt man ifn Sratle (falcula), beflei= 
Det er bas Ende wie ein Schuh, fo heip̈t er Şuf ober Sil a ue (ungula).

Bom inneren $\mathfrak{B}$ au ber Săugethiere muś als (Figentritm=

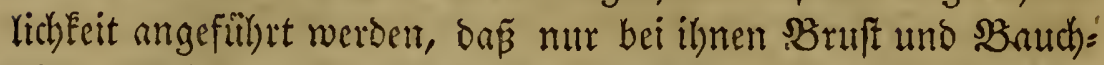
holle dutch einen flachen Muślel (bas 3 merd) fell) vollfom= men getrennt find, bei ben librigen Nitcfgrattbieren aber mekr oder weniger zufammenhaingen. $2(m$ obern Ende ber $\mathfrak{a} u f t=$

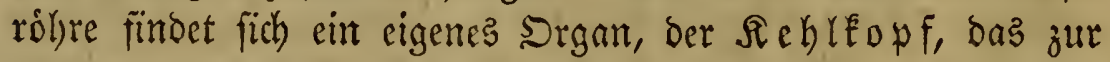
Servorbringung ber Stimme behitflich iff. Sonft finben fich

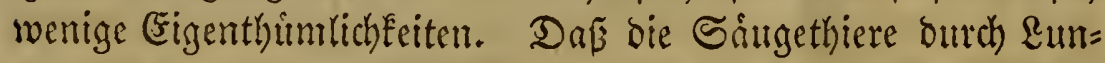
geir athmen, ba $\tilde{\beta}$ bas rothe etwa $30^{\circ}$ warme 3 hut aus ber Entrige erft wieder ins Şerz, welches aus 2 Sammern uno 2 Burbofen befteht, zuriekefebrt, ehe ez in ben Surper fich ver= breitet, uno bar fie lebendige sungen gebiren, welche bie

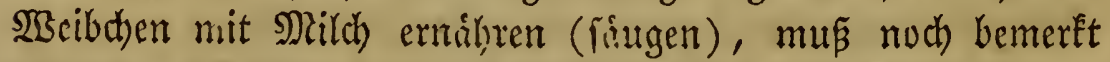
werben. Die Srgnne, weldbe bieje Mild) bereiten, uno 3itgen ober (5) uter heisen, liegen bald mebr an ber Bruft (2ffen), bald am untern (Ende Dez \$auďes (Nimber).

Man Eennt gegenwårtig etwa 1000 verid)iedene 2(rten von Sittigethieren, bie wieber in mehrere Scanptgruppen fich brimgen laffen, zunad)ft nach der sBitbung ber 3ehen in 3, wie folgt:

\section{A. Nagelfaugethiere. Shre Behen Erfte Dronung.}

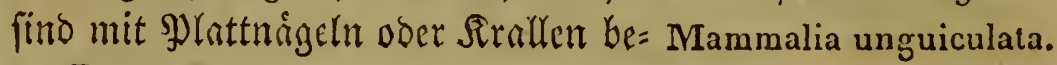
swaffnet.

a. Mrit allen brei 3abnarten.

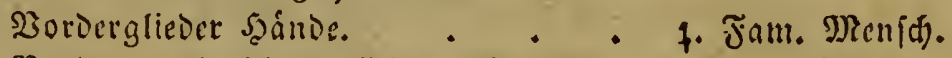

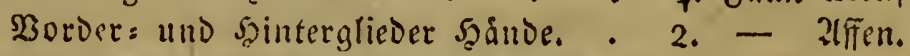

2orderfúpe und Beben Durtil eine Flug:

baut verbundert. . . . 3. - Jredermáufe. Sorder = und Şinterglieder Fúfe.

Shne einen Sack un Die Biter. 4. - Paubtgiere.

Mit einem ভack, un Die 3ikęen. 5. - Bentelthicte.

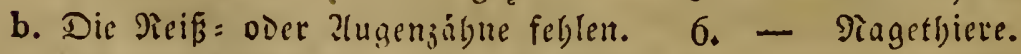

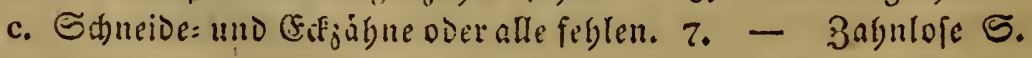

B. Scuffátugethiere. Shre 3eken= 3weite Sronung. fpisen find von Scufen bef́leibet.

a. Cine 3ebe an jedem Fuf. . . 8. Fam. Dferde. 


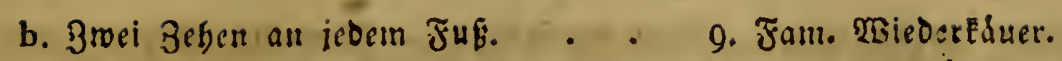

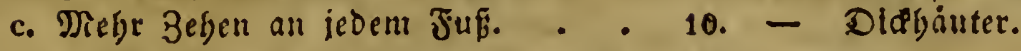

C. Floffenfáugethiere. Shre Be= Dritte Dronung. bet find ourch eine Sdrwimmlyaut Mammalia pinnata. berwadjjen.

a. Mit 4 foffenfirmigen Füßzen. : - 11. Fam. Seegunde.

b. Mit 2 floffenformigen Jißfen. . . 12. - Walffifde.

\section{Erfte Familie. 3weilinder, Bimana.}

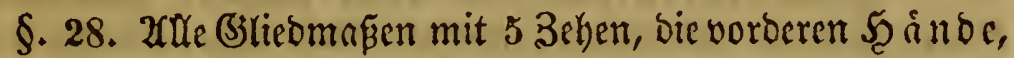

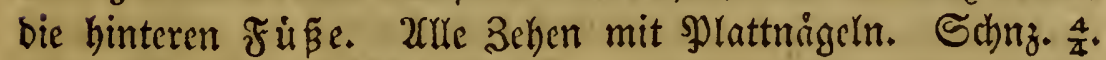
(Fif;. $\frac{1,1}{1,1}$. Badf. $\frac{5,5}{5,5}$ ).

Slerber Die einzige (5attung Des Menfden (Homo), welde von

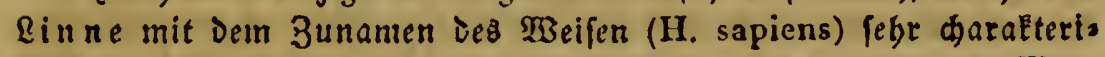
frifd) befeidnet wurde, Denn ble $\mathfrak{B}$ er $n$ unfe lftes, melde den Den: fiben von allen sbieren volftandig unterfdeibet, wabrend pein Rob: per mit Dem ber Thiere vielfad úbereinftimmt. -

Der Tenid wird fegr unbebolfen geboren, erbalt am Ende

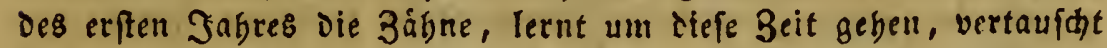
im adten Jabre feine 3 álune mit neuen (fdidgtet) und erbalt in adtzebnten bis zmantigften Jabre feine Irkten 4 Wadgabne (Tois: beitgaigne). Jm 24ften Sabre ift er erwahien und gat nun bie Sobe you $5^{\prime}$ und $\dot{5}-7^{\prime \prime}$; Eleinere und grifiere Denface fino un: gemignlla. Er gebt allefn yon allen Thieren aufredt, bedient fich alleir Der Spradje und gat allein eine bernúnftige Seele. Er ges Deibt in allen 3onen, und zerfallt nad feiner angebornen serfdie: Denbeit in 5 șauptftanme.

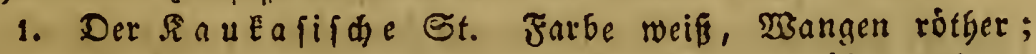
Stiru hod, fanft gewilbt; Raje giemlid fentred)t, máßig, fpikger; Sinn zurúdgezogen; Şaare weid, gelodt; Zart ftare. - Ve:

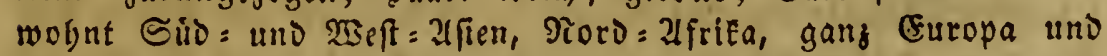
alle you bort aus bevollerten Biegenden Der Eroe.

2. Der Jiongolifhe Et. Zarbe gelb; 2lugen fidmarj: braun, Elein, fbief nad oben gegogen; Waangen Gervorragend;

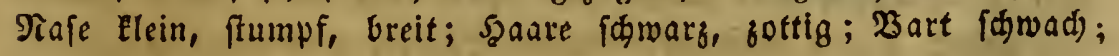

*) Durd Dicfe Formel bezcidnet man im Surgen Die Zabl ocr

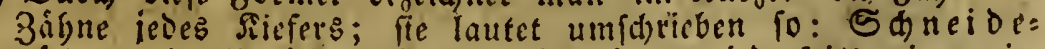

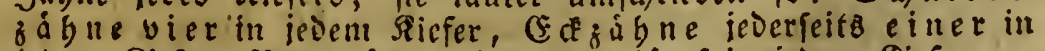

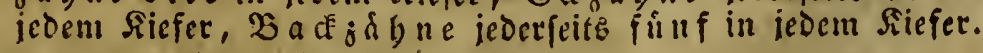

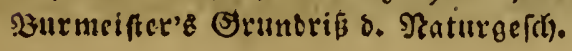




\section{Erfter 26b/dnitt. 20ologie. 1. Rüçgeattbiere.}

Siefer gurudfegogen; Sinn yorftegeno. - Bewognt Mittel : und Dit = 2lifen bis gegen Niord slmerifa bin.

3. Der $\mathfrak{x}$ merik a nifhe St. Farbe rothbraun; Befidts:

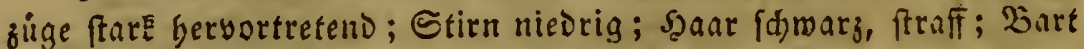

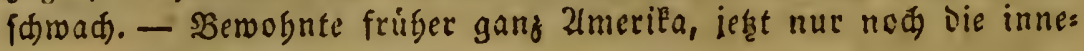
ren unEultivirten B̈egenden.

4. Der $\mathfrak{R a}$ a layifale Et. Farbe braun, balo beller, balo

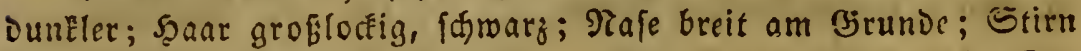

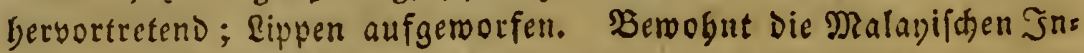
feln und 2tuftralien.

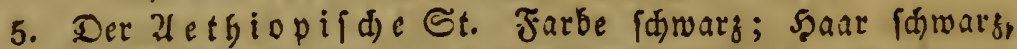
Fraus, wollig; Stirn flad), geneigt; Fafe Elein, ftumpf; Sippent ftarE aufgeworfen; Der fiopf nad Dem Edeitel wie jufanmenge: Drudt; ßiefer bervorrageno. - Bemognt Mittel = uno Sird: Xlfs

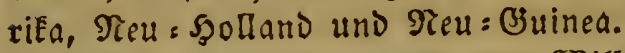

Man nimut all, Dá 1000 Millionen Renfden auf Dér Ere De ivobnen, und Daf́ alle 33 Jahre eine Beneration ausfterbe, wábrend etra $\frac{\pi}{8}$ mebr geboren werven. Dies giebt für jecie MRi= nute 63 Todesfálle und 70 Geburten. Die Rafrung Des Mens fohen ift gemilat theils aus Dem Pfanjen=, theils aus Dem Thiers reid. Siufernbewogner und grordländer effen megr Jielíd; Die bes wogner Der beigen Zone megr Pfanjenftoffe, bejonders Frủmte. -

3meite Familie. Sierlyander, Quadrumana.

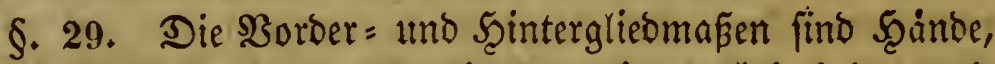
beibe meiftenz fúnfzebig uno mit झुlattnageln, bei einigen mit

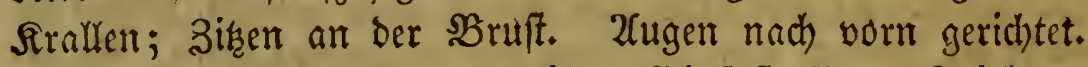

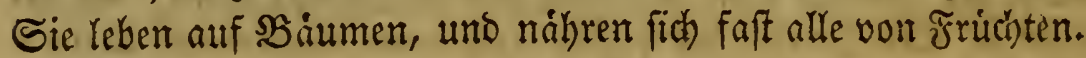

Die 2 ffen, welche in biefe Familie gehoren, find in ihs rem Rórperbau bem Menjoyen am ähnlichften; bodh untericheis ben fich bie meiften fiton Durch ben langen Sthwanz, und alle burch bie Bebaarung bes Rorpers. Sie leben nur in ber beis

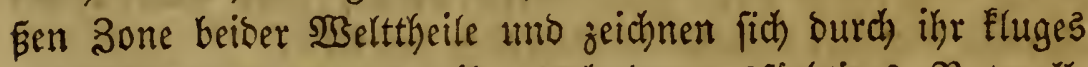
und liftiges Betragen, igr nachal)mungsfítchtiges Raturell,

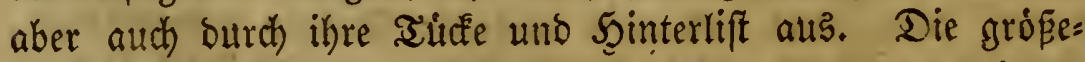
ren 2rrten haben auferorbentiiche Stärfe, uno merben einjel= nen Menifhen gefákrlich; fie vertheibigen fich ourd $\mathfrak{T e r f e n}$ mit barten Fritchten, Schlagen mit Snitteln, uno viele auth ourch sBeisen. SBefonders thum bies bie burch ein gropeses

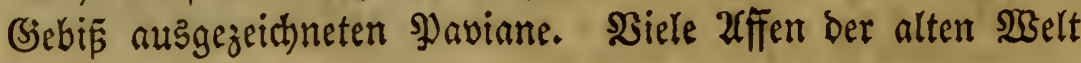


baben ein nactes Serä́a uno Infiben an ber innern Seite ber $\mathfrak{B a n g e n}$ (Bactentaj(hen), beibes findet fich bei benen ber neten $\mathfrak{B}$ elt nicht.

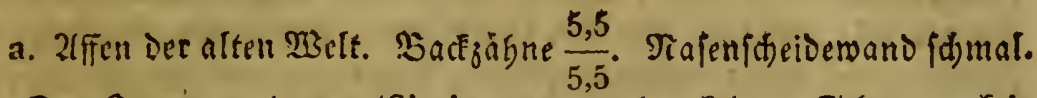

Der Draing ut ang (Simia satyrus) bat Eeinen Etrmanz, Keis

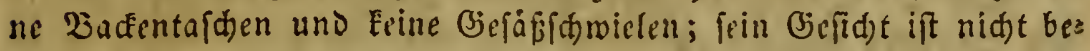
baart, uno Die Saaare am Sorderarm ftefen rúchwart?. Er lebt auf Sorneo, mito bis 5 lyod und bat cine Eaftanienbraune Jarbe; jung befirge er ein leidtes Dem Des Menfden nidt unábulides Oes

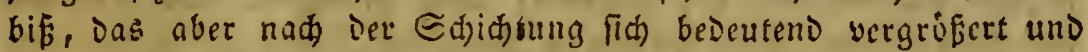
pavianzartig wiro. Der alte 3iffe beifft bann \$1 ongo. -

Der (ji $660 n$ (Hylobates lar) bat ebenfalls feinen Edman uno Eeine Daffentafbun, aber Elcine Befábiddmielen und fegre lange

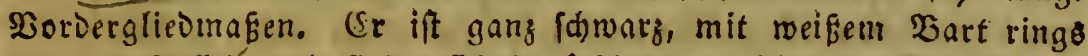

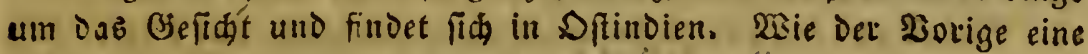
Eeltengeit in Sammlungen.

Der gemeine Pavian oder Mandrill (Cynocephalus Maimon) bat einen nur fefre furgen Edmans, grofe breite befáf: fdmielen, Backentafden, ein ftarE entwičeltes bebif mit grofen Eafjágnen. Geine Jarbe ift grünbraun, mit wriflibem Siunbart, feine 2zangen fino gefurdit tho blau, feine Nafe roth. Er twiro bis $5 \frac{1}{2}$ Juß Gáufig yor; er ift willo uno unbándig.

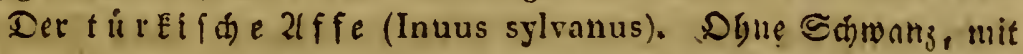

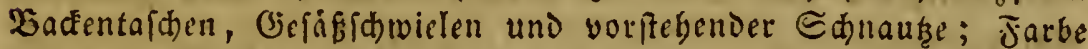

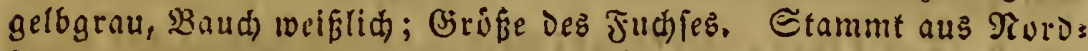

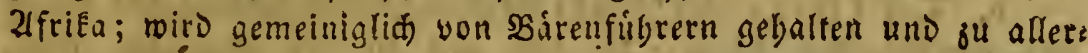
Iei șunfftúcken abgerid)tet.

Die gemeille Meereate ober Der Mogenafe (Cers

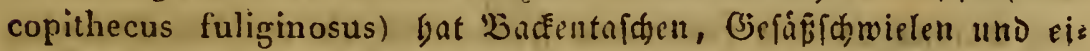

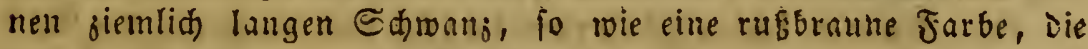

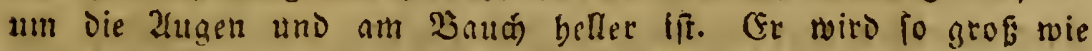

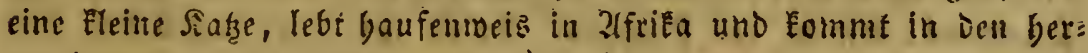
umziegenden Pienagctien am bảufiglen yor.

b. 3u Den.2lfien Der neuen SBelt, Denen arfo Badfentajalen und

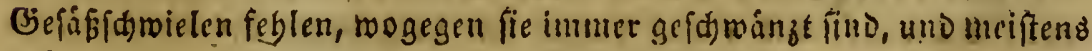

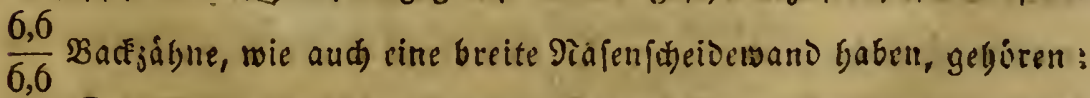

Der $\mathfrak{i}$ úllaffe (Mycetes Beelzebul). Er bat eintr féfe breiten Innterkiefer, eine Enúderne Paufe an Zungenbein, uno eis

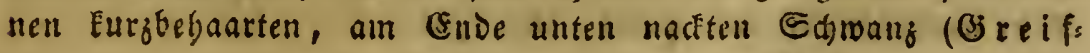




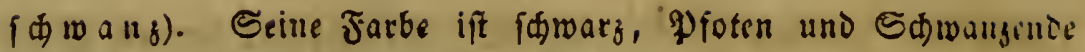
rotgbraun. . Brifife einer Rakge. Brafilien.

Der $R$ a p u gine raffe (Cebus capucinus). IRit langem, iberall

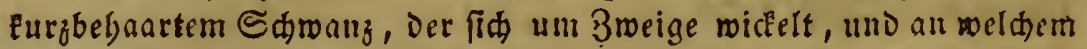

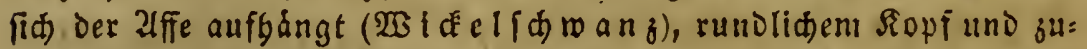

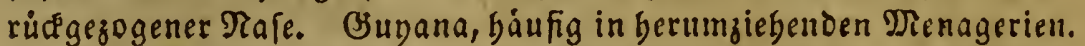

Der Seidenaffe oder Sachus (Hapale Jacchus). Şat eis nen Backgabn reniger als Die úbrigen 2lfen ber neuen Melt, nebm: lid) 5 an jeber Seite, und Rrallen an allen 3egen, mit ?lusnabme Des Daumens. Seine Farbe ift farmarigrau, an iedem Dhre ein grofier Şaarbúfhel, fein હdwang meif geringelt. So grof wie ein (Eid)

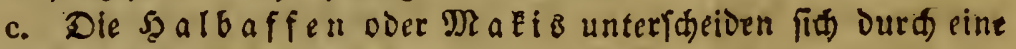
fpiţe bervorftebende Gdjnause, uno ourd Den Sirallnagel am 3eiges finger Der Sinterbảnde, wábreno alle bibrigen plattnágel fino, von Den eigentliden 2 ffen. WUle leben in ber alten $\mathfrak{B e l t , ~ 3 . ~} \mathfrak{B}$.:

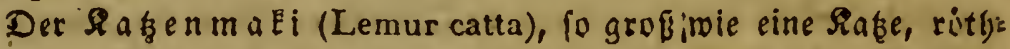
lichgrau, mit weißem Baud, und weiß und fकwarz geringeltem Echmang. 2uf Madagaskar.

\section{Dritte Framilie. Flatterer, Chiroptera.}

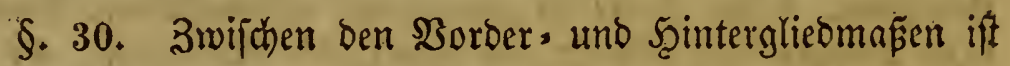
eine grope Flughaut aubgeipannt; Biken an ber $\mathfrak{B r u f t}$; (5ie-

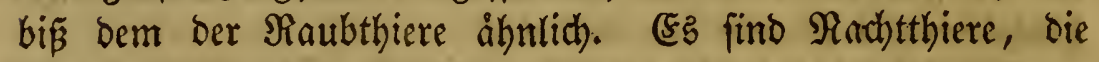
von Infeften uno Eleineren Rüfgrattbieren, auch Friethten, fici) nåbren.

a. Der fliegende $\mathbb{R}$ a $\mathrm{El}$ (Galeopithecus rufus) gat 3eben son gemógntider Range, bie alle mit in Der Flughaut fiłzen, $\frac{4}{6}$ Ed)8. Die untern gekerbt uno $\frac{5,5}{5,5}$ Back8. Cre bat bie Bróbe eir nes Soanindent, eine rothbraune Farbe, und lebt auf Den Moludén.

b. Sei Den áden Fledermáufen fino Die Befgen Der $\mathfrak{B} 0$ r. Dergliedmáfen fegr verlängert, und fecfén, mit 2lusnabme des Daumens, mit in Der Jlughaut, Die fie fpannen; 3efjen ocr Jiin= terglleder frei. Dabin:

Der fliegende 1 nd oder $\mathfrak{Z}$ ampyr (Pteropus edulis), ogne Sdyanz, mit einem Nagel am 3eigefinger Des Dorbergfied:

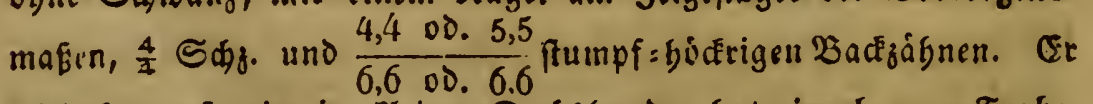
wird fo grố wie ein Eleiner Dadblyund, bat eine braune Farbe, und lebt auf den offludifden Snfeln. DaßB er Edrafenien bas Blut ausfange, ift eine Fabel, bies thut 
Die $\mathfrak{B}$ I a f $\mathrm{n}$ a fe (Phyllostoma hattatum), mit einem Eurgen

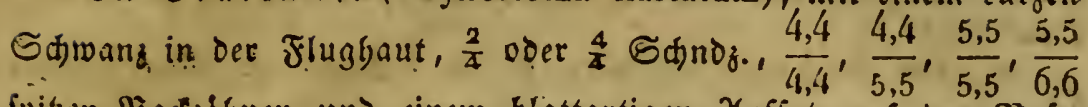

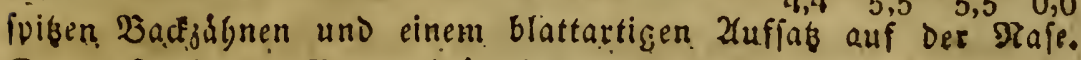
Se grof wie eine Ratte, briunlid); Yebt in Brafilien.

Die Szafe $i$ fen $\mathfrak{n}$ a fe (Rhinolophus ferrum equinum) bat el=

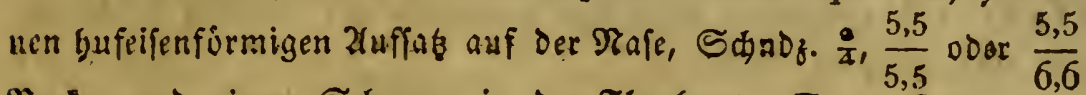

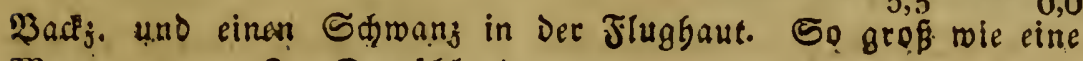
Diaus, grau. In Deutfdland.

Die gemeinen Fledermáa (Vespertiliones) baben Reis

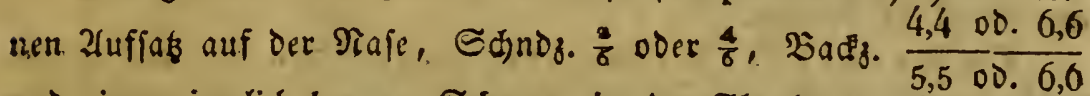

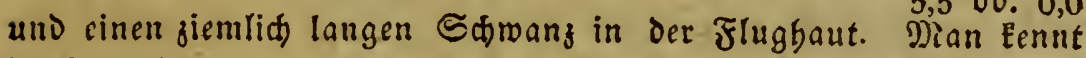
in Deutfdilano gegen ein Dukent Zrten, unter welden bie lang: ofrige F̧. (V. auritus), mit DGren yon boppelter Sapflánge, am mertwirdigien iff. Die Fledermsufe verfallen Den $2 B i n t e r$ bin: burch in einen Edhlaf (Retbargie), und verbergen fid Dann, mie aud am Tage, in aften Bebauden, Mauerlónern, goblen Báumen zc.

\section{Bierte Familie. $\mathfrak{R}$ aubthiere, Ferae.}

§. 31. Sorber = uno Şinterfúpe gleid)gebilbet, mit glei= (hen, won. Srallen bewehrten Behen in verichiebener 3aht.

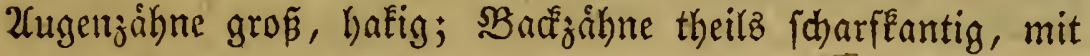

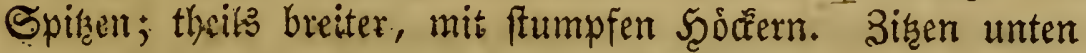

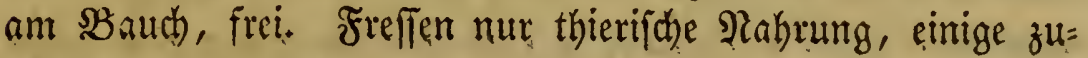
gleich) audb) Frưthte.

A. Die Fleifh) freffenoen $\Re$ aubitiere (Ferae carnivorae) baben ftets $\frac{\sigma}{q}$ Sthneibezålhme, eimen ftarken (Fd = zahn, Eeine Schlüfelbeine uno einen gropenen אörper.

a. Dix Gundartigen Raubthiere (Canis) baben untemeg=

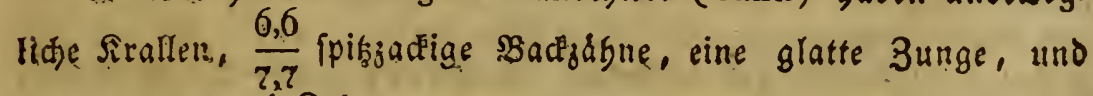
yorn 5, Ginten 4 3egen.

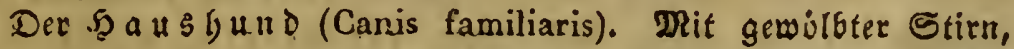
oft hángenden Obren unl aufmártz gebogenem ङømanz. Heber: all Begleiter Des Menfiden in vielfaden 2 barten.

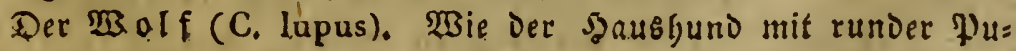
pille und gradem, Gängendem ङduman; gelblidy yon Farbe mit

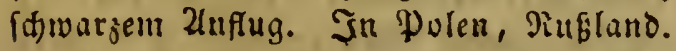

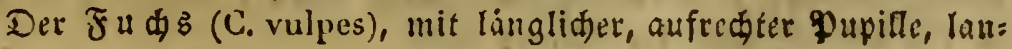




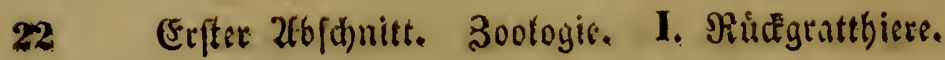

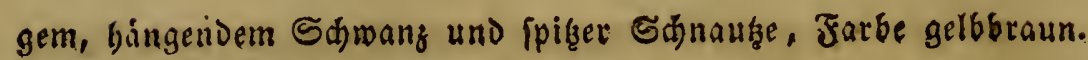
Deutidfarid.

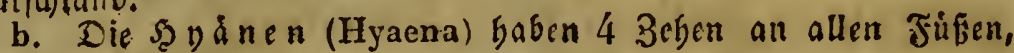
$\frac{5,5}{4,4}$ Bad̆. Eeine berweglifien Sirallen, aber eine raube 3unge. Maan Eenut 2 ?teten, Die geftreifte (H. striata) und bie $g e=$ fle de (H. crocuta); beide fino graugelb mit bunelen 3eidnun= gen uno leben in 2 ffrita.

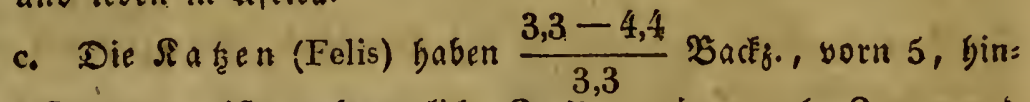
ten 4 3eben, meiftens beweglide Sirallen, eine rauge 3unge uno cine finglide Pupille.

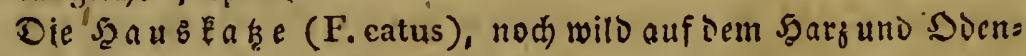
walo, Der Róme (F. leo), Tiger ( $F$. tigris), Jaguar (F, onca), Der (Suguar (F. concolor) und viele andere geburen bieger.

d. Die 3 i b ct $\mathfrak{g}$ a eine grof̧e żmeifaderige Drífentafde unter Dem 2 fter, 5 3elen an allen Jûnen und eine raube 3unge. Farbe grau mit fdmargen

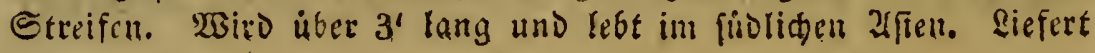
Den 3iveth.

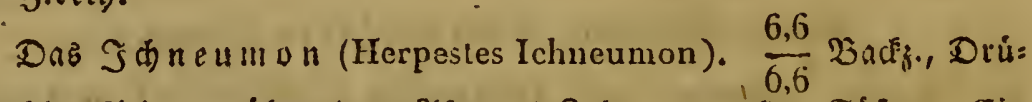

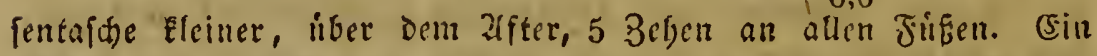

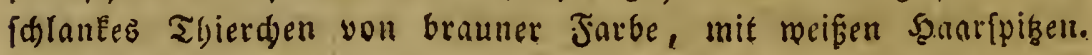
Sn Zlegupten.

e. Das Stinktgier (Mephitis putorius). Şat $\frac{-4,4}{5,5}$ Back neben Dem Ifter 2 Drifen, aus weldjen ez cine fegr ftinkente

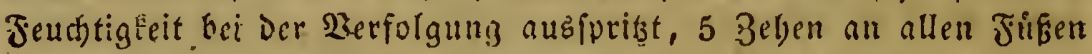

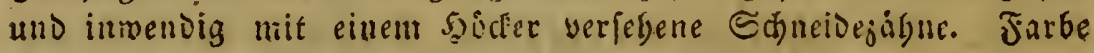

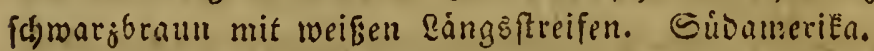

Die wiefel (Mustela) Gaben einen fegr langeftreden Sírper,

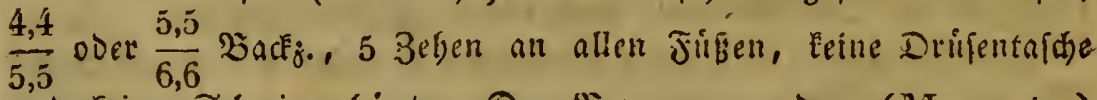

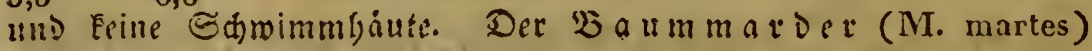

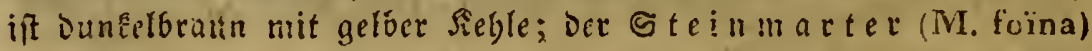
ift bellbraun mit meißer fiefle; der $\mathfrak{S} l$ ti s (M. putorius) gans fdrmarzbraun mit meiper Edhnautse; Das jeettchen (MI. furo) bell ofergelb; Der 306 er (M. Zibellina) braun, Siopf uno Gurgel

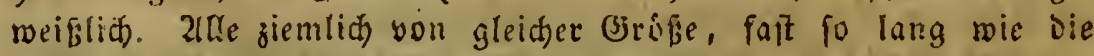
Sillse, aber writ fd)lanfer mit Eúrzeren Beinen. Der $\mathfrak{B B}_{3}$ iefel (M. vulgaris), orittel fo grofi, bellbraun, Siegle meis. Der Sgermelin

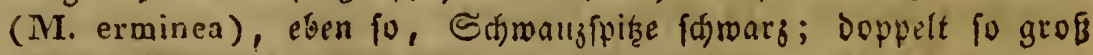
alo ber sistefer; miro in $23 i n t e r$ mei巨. 


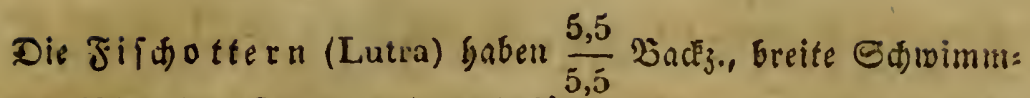

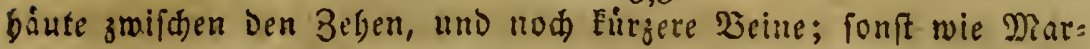
Der. Bemeine Jif dotter (L. vulgaris), wie cin tüdtiger Sia. ter, rithfidgbraun. 2(n Jifdteiden.

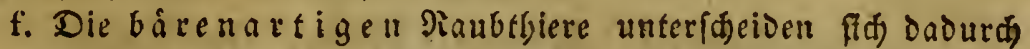
yon Den úbrigen, Daf fie mit Der ganzen na díten Gobje aufteten (Plattfuß̧gánger, plantigrada, find), mágrend bei Den übrigen die Sogle belgaart ift, und nur die Belyen Den Boben berifgren. Sgre

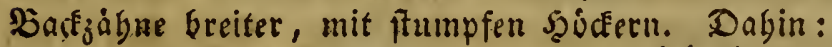

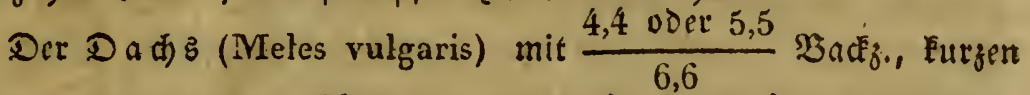
Beinen, gedrungenem Ssorper, einer-Drúfentafde úber Dem $2(f t e r$,

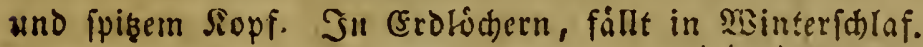

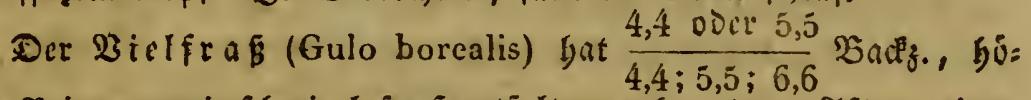
bere Beine, zmei fifmierlofe şautfalten neben Dem 21 fter, einen

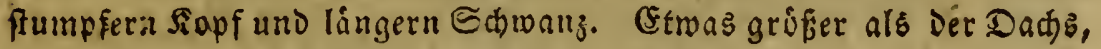
fămarzbraun, mit getlerem Rúč́en. Smr গiorden.

Die $\mathfrak{B}$ åren (Ursus) gaben $\frac{4,4-6,6}{4,4-7,7}$ Bacḱf.e einen runden Siopf

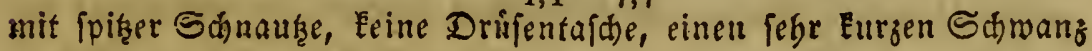
uno bobere beine. Der braune $\mathfrak{b}$. (U. arctos) \{đmarzbraun;

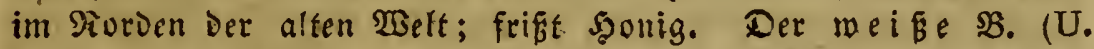

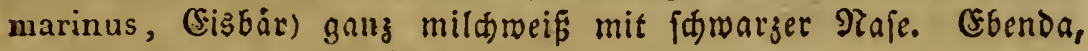
aber nur an Den אinjen.

B. Die Snieften frefienden $\Re a u b t h i e r e$ (Ferae insectivorae) fino flein, fein gebaut, mit fpifzer, hervorragen= ber Sd)nauke, fhwamender Zahl ber Schneidezáhne unb oft

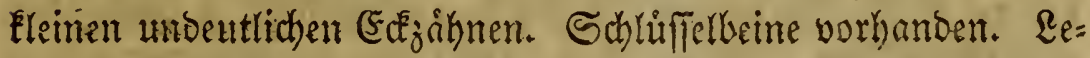
ben von Snfeften uno $\mathfrak{B}$ urmern, Gichfter: Eleinen Marufen.

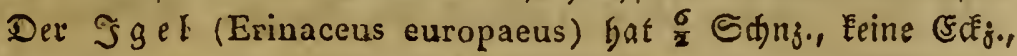
$\frac{7,7}{7,7}$ Backg, eine gelerbse Tafe uno viele oura eirander geftecte Étadgeln. Er Eann fid Eugeln. 1' lang; fríst MRáufe.

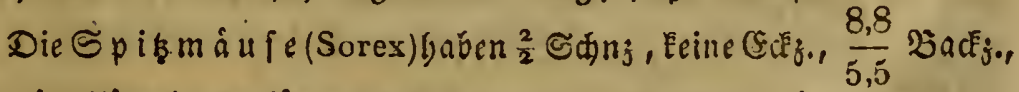

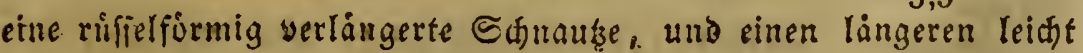

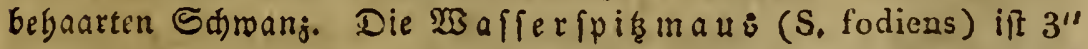
lang, fowarg, unten meis. 2lu llfern. Dic 3 wergfpitgaus (S. pygmaeus) ift $\frac{3}{4} "$ Inng $_{2}$ graubraun; das Rleinfte Sángetbier.

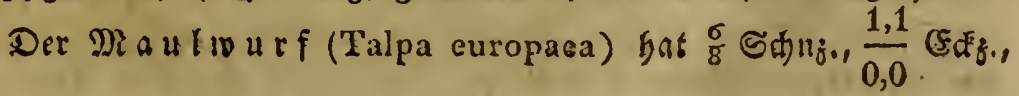




\section{Erffer abfidnitt. Soologie. I. Rütgrattbiere.}

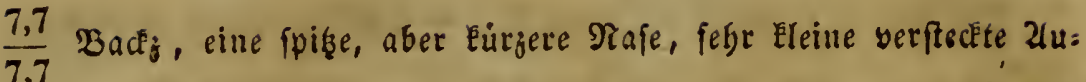

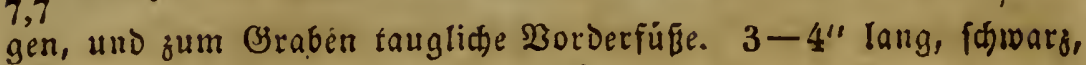
wüblt in Der Eroe. Frifft Regentwúrmer.

\section{Funfte Familie. Beuterthiere, Marsupialia.}

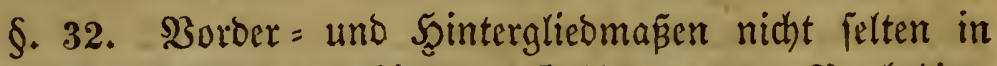

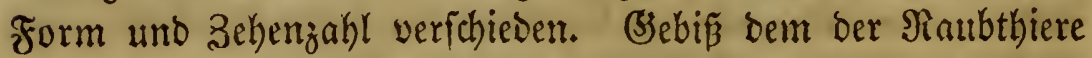

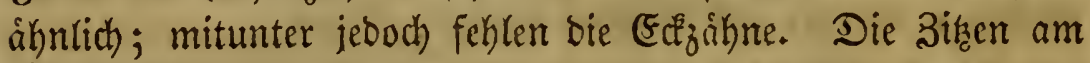
Ḃauch von einer $\mathfrak{x} a f$ che ober Scautfalte umgeben, welche bie faugenden Jungen umichließ̧t und ichuhst. Dabin:

Die $\mathfrak{B} e$ utelrat te, oas Dpoffum (Didelphys virginiana), fo

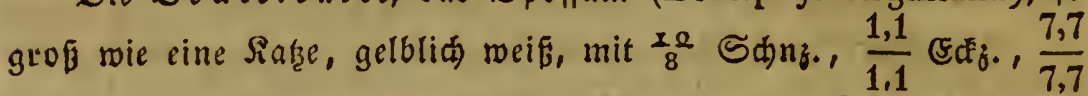

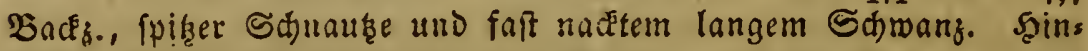
terfúfe handartig mit nagellofem Daumen. Frifft 20 igel und be= ren Eier. Trágt Die iungen im 'Beutel. 'Braftlien.

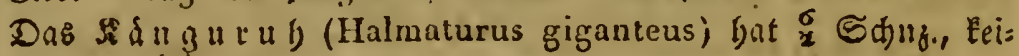

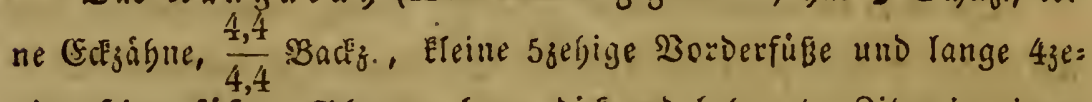

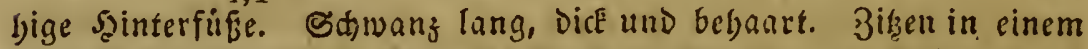
grofert Beutcl. WBird 4' hod, ift afdgrau, frift mur Siráuter und lebt in Tentoltand.

\section{Sechste Familie. Ragethiere, Glires.}

§. 32. $3 w e i$, felten oben vier, Schj. in jeobem Siefer,

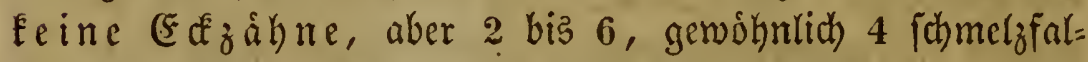

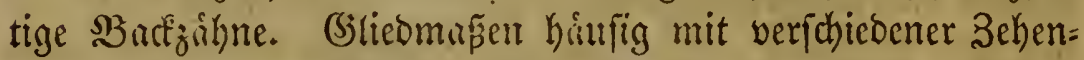
jahl, bie hinteren langer, Bižzen am \$auch. Freffen \$flan= jennabrumg.

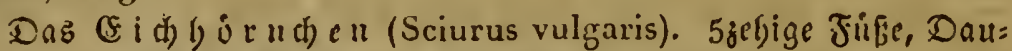

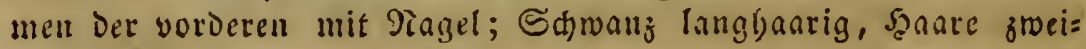

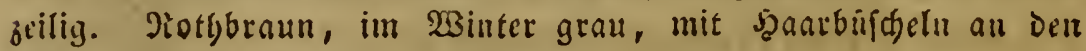
Shret.

Dor Siebenfdláfer (Myoxus glis). Ție Sorigeb, aber

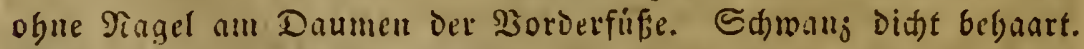
Gran, mit brannem ?lugenEranj, Brófe Der Tiatte. Sn פisálderit.

Das $\Re$ urmelthicr (Arctomys marmotta), mit Eurjen, ab: gerundeten Dhren, Eurrzcm, belaartem Echrwanz, und gelblidgrau:

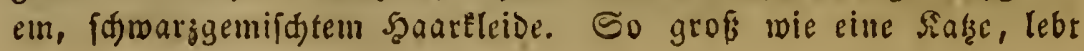




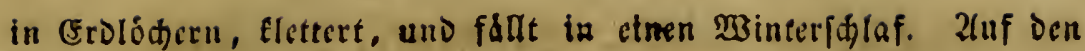
Illpen, befonders in Savouer.

Der $\mathfrak{S}$ a $\mathfrak{m}$ fter (Gricetus vulgaris), mit eurgen, naciten $\mathrm{Ob}=$

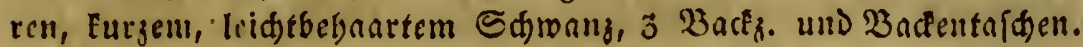
Srobe Der Fiatte, gelbbraun, Siegle und Bruft fdtwarg, gelb ein: gefufft. Thiringen, in Erolidern, fallt in Minterfalaf.

Die If \& ufeg a t tung (Mus) bat Eurze, nactite Shren, lange, reid,tbebaarte, fduppige Sdivdnze, 3 Badk., aber teine Baden: taffen. Die $\mathfrak{i s}$ an ocratte (M. decumanus), gelbgrau, Die

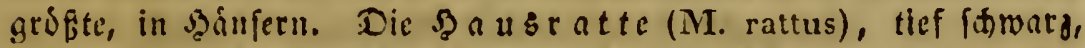
Juife fleifdfarben, nadt, Eleincr; ebenda, aber von jencr faft ver= Drángt. Dic Sg a 11 s ma u \& (M. nusculus), inhufegrau, Eaum 2" lang; gemein in Solufern. Die 2 ranomiaus (M. agrarius),

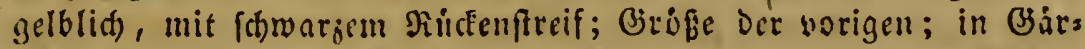
ten. Dir $\mathfrak{i B}$ a l $\mathrm{d}$ m a u : (M. sylvaticus); gelbgrau, obue Rúclen= ftreif; in Sisardorn. Itlle gemein.

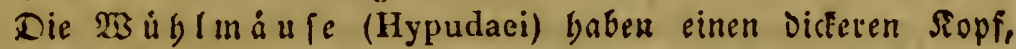
felbr Eleine Obren und einen Eúrzeren Samang. Die Jeloma us (H. arralis) ift graugelblid) uno to grof wie eine Maus; grábt Biange und Rider auf Jeldern. Die שzafferratte (H. amphi-

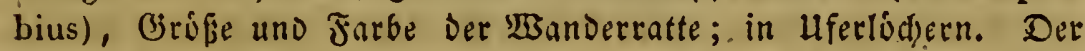
Le mu ing (H. lemmus) bat cinen febr furzen Sdmanz, gróbere Jirallen; Jarbe braun, gelb gefledt; in Sibirien; maḍt fidnut: grade $23 a n d e r u n g e n$.

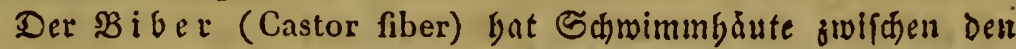
Sinterbeben und einen breiten, mit ভdjuppen bedeckten હdjwang. Jarbe gelbbraun, Range über $2^{t}$. Zlın ufer Der Jlitiffe, no er Wognungen baut, befonders in Tioro = 2tmerita.

Dns Stadelfdwein (Hystrix eristata) gat Eurze Dgren, eine raufe 3unge, viele lange Drebrunde Stachelu und vierjebige

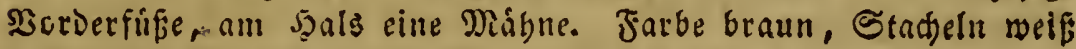
geringelt. Eird: Europa, Mord: Ifrita.

Die 5afe S.dno\}. im DberEiefer, 2 grofere sorn, 2 Eleinere Dabinter uno

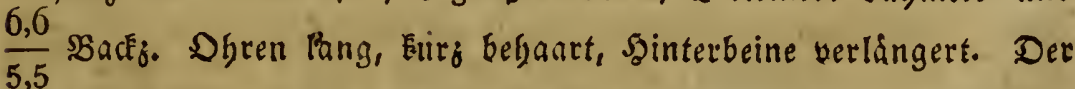
ge meine $\mathfrak{S} a$ a fe (L. timidus), Obren línger als Der $\mathfrak{s} p p$, Far:

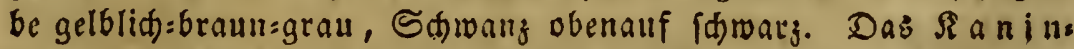
wen (L. cunirulus), Gellgrau, Ogren Eurrzer als Der Sopf. In Erolódern, bie uno ba nidt felten.

Das Meerfdreinden (Cavia cobaya) Gat eurje nadte

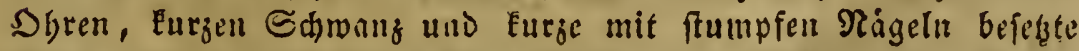

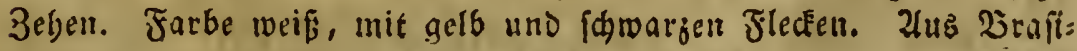
lien, mo man's faton gezáant fant. 


\section{Siebente Familie. $3 a \mathfrak{h n}$ lofe, Edentata.}

§. 34. Schneidezäbne fehlen, bäufig auch die Ectzaibne, bisweilen ferbft bie Baclzäbne. Báhne einfórmig, bafig, bis=

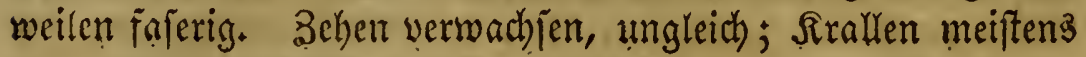
fehr groß. - Şierther:

Das Faultbier (Bradypus), mit EdF. und $\frac{4,4}{3,3}$ cnlinori= -

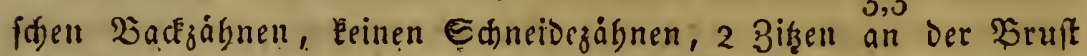

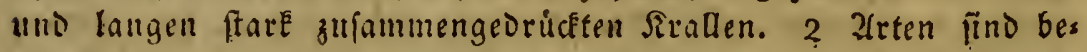
Eannt: Der ? i (B. tridactylus) mit 3 Beben an allen Juifen, 9 Şalemirbeln und obne (sckjábne; und Der $\|_{\mathfrak{n}}$ a $u$ (B. didactylus),

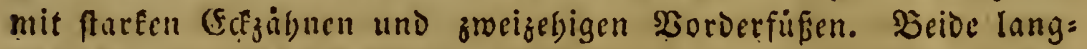
fame Fbiere, Die auf Bảumen leben.

Dab Bs irteltgier, If rmadilt (Dasypus), mit vieken $(8-25)$ cylindrifden, oben zugefdärften baacfzágnen, aber olyne Ea" und Sdneidezibue. Qeib yon einem Szornpanzer bedeckt, bins

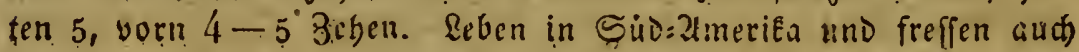
Snfetten. 3 B. Der Tat (D. tricinctus), mit Eurzem Sd wạn, und wenigen breiten Birttern.

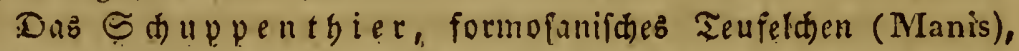
bat Eeinę 3ábne, aber brẹitc, Den ganzen Eeib bedeckende Eduppen, grwifden meld)en, uno an Baudh, fparfame Sgaare. Dian Eennt 2 2ltten, eine (MI. brachyura) mit Eurjem Sdymariz uno 5 Bebeu an allen Jifien, Die andere (M. macrura) mit langem $S_{\mathfrak{c}}$ manz und vorn 4 3eben. Beide in Dftintien.

Der 2f meifenfreffer (Myrmecophaga) bat keine 3äbna, ein Eleines Maul, eine lange murmfórmige 3 unge und lange Şaare.

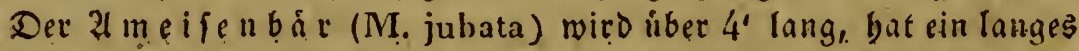
braunce, an Den Seiten belleres Şaar uno wognt in Süd=2umeriea. Frif̧́t nur zlmeisen.

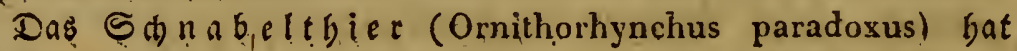
einen naikten, yon fleifdiger Saaut bekteideten Entenidhnabel, $\frac{2,2}{2,2}$ fa: ferige Backjálgne und Edjmimmbåute jmifhen den 3ehen. Es miro $1-12$ lang, ift braun, und lebt in Den Jhitifen und Eeen Nieus bollanos. Nad Ceinigen foll es Cier legen, Dods feglen ibm bie Bizen nicht.

Xchte Familie. Finfufer, Solidungula.

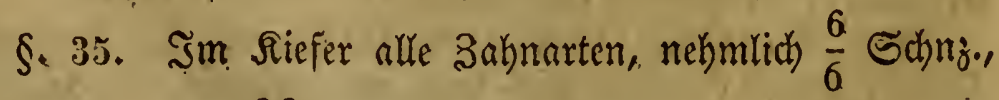

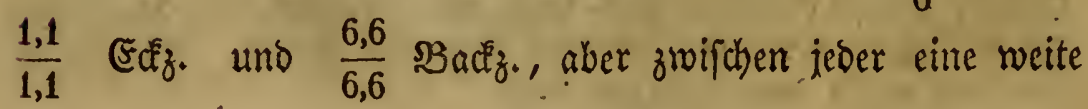


Erffe Sinffe. Einugetbiere. Eingufer. 3weibufer. 27

Lúfe, Eafjăhne fehlen mitunter. Füüe einjehig, mit gro= Ben Scufen.

Şierlyer bie cingige Battung Des $P$ ferdes (Equus), woson 6 ? lefen betannt fino, nefmlid das ge me ine $P$ ferd (E. caballus), Der (Efel (E. asinus), Der \$a a f e f f l oder Didhiggetai (E. hemionius), Das $3 \circ 6 \times a$ (E.Zebra), Das $\Omega$ u agga (E. quagga) uno Der $D$ na $g g a$ (E. Burchelii), alle leben urfprúnglid in Der al:

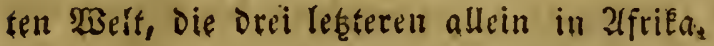

Neunte Fanilie. 3 weihufer, Bisuica, voer MBieder áa uer, Ruminantia.

§. 36. Seine Schncioejăhne in Sberfiefer, aber 6 ober gewubntich 8 im Unterfiefer, allermeiftens feine ober

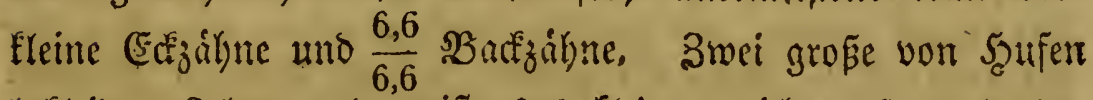
beflciocte 3eben und meiftens 2 Eleinere nid)t auftretende or= binter. (affterflauen). Die bierber geburigen abiere frefien, wie bie vorigen, mur \$flamjennalrung, bie fie ungetautet ver= fhluten, balyer bicfelbe aus bem Magen wieber hernufferigt, 'un jum zweiten Male gefnuet zu werben. Deshalb beftegt

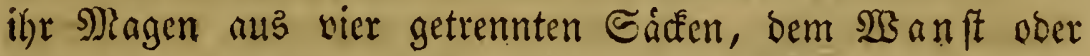
Danfen (rumen), Dem Resmagen ober Der Scaube (reticulum), bem $\$$ Parter boer $\mathfrak{B}$ uch (om sum) uno rem $\mathfrak{L} \mathfrak{a} \mathfrak{b} \mathfrak{m a g e n}$ (abomāsum). Şierher:

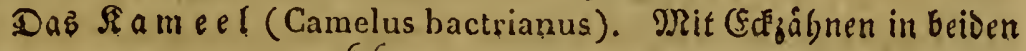

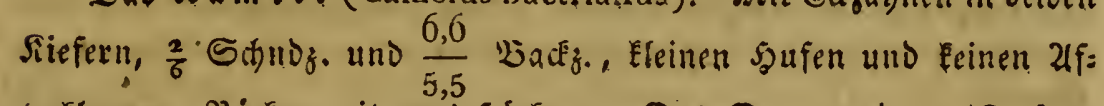

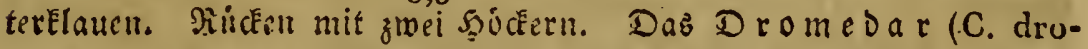
medarius) bat nur eincin saiffer. Beite nidst megr wilo, aber in

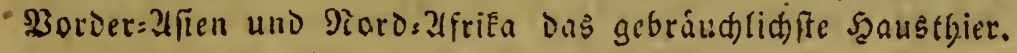

Das la a m a (Auchenia Lama). MRit eben fo vielen 3ágnen,

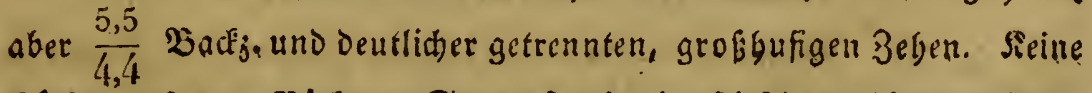

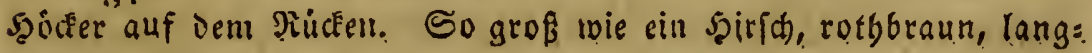
baarig. Peru.

Die B̈iraffe (Camelopardalis giraffa). DGne Ceckänne uno IlfterElauten, aber mit Eurjen yon Der \$ant EeEleideten Stirngor: nern; Sals lang, Szorderbeine lánger uno biber. Jarbe gelbfit, rotgbraun geflecḱt. Ifriéa.

Die Siridgattung (Cervus) bat bismeilen Cadf. im Dberfiefer, und beidjnet fïh Dur由 das gube, áftige Öemeil ber 
DRannch, Das alle Jabe ecneuert mird, aus. Bor bem Xuge cire lánglibe Bertiefung ( phus), rotbbraun, Dánnchen mit großem, vielgadigem Betweih. Das $\mathfrak{R}$ 占 (C, capreolus), graubraun, Daknnen mit Eleinerem, flar: biderigem Bereif). Das $\Re$ en ntgier (C. tarandus), ber Dam mbirfd (C. dorcas) und das Glentbier (C. alces) gez buren aud bierber.

Dle al ntilopen (Antilope) baben meiftens bie fiblante Be: ftalt ber Spirfde, aber bié yon Şorn ủberjogenen, nidt \&ffigen Bie. meige der Rinder, wiewogl fie barter und feiner find. Sn (Furopa nur eitu 2trt, bie çs mfe (A. rupicapra), fdmarjbraum, mit wei= fem Sopfitrid) und bakigen Đórnern. Sdameis, Tyrol.

Die Blege 1 gattung (Capra) bat eine tiefgeipaltene Dber: lippe, Eantige Sórner und einen $\mathfrak{B}$ a $x \mathfrak{t}$ am Kinn; Dabin: Die $\mathfrak{g}$ em. 3 iege (C. laircus) mit Purzen, fanftgebogenen Sornern; Der Steinbod (C. Ibex) mit gropen, fturfen, Enotigen bornern, ม. b. a.

Die 5 da afgattug (Ovis) bat nach augen geridtete, eno: tige Sjorner, aber Eeinen Bart. Dab gem. S d a a f (O. aries) bat einen Eurken Shroanj und ein weides, eraufes Wollbaar; es finbet fid ridt mebr will.

Die $\Re$ indergattung (Bos), bat runde, meiftens Doppelt ges bogene Şurner, eine braite widht bebaarte Sduaube, Purje Szaare, aber einen Sgabbufdel ain Sdyanj. Dabin: ber gemeine $D$ d) (B. taurus), mit flacher Etirn, grofer Wamme uno ciner Laifte zmi= fden ben Jjornern; ber $\mathfrak{I}$ u er o d 5 (B. Urus) , mit einer wolligen Dágne an Sgalfe, uno Dart am Finn. Der Büffel (B. bubalus) mit Eurgen fteifen Dünnen Şaaren, etwas gemolbter Etirn und tur: zen biden bürnern. Dptindien; auth in Uugarn und Stalien gas z̊̊̆́mt.

3ehnte Familie. Bielhufer, Multungula, doek Didéláter, Pachydermata.

§. 37. Mebr als zwei auftretende 3eben mit Elcineren

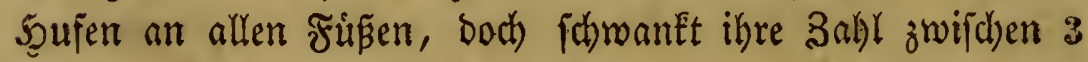
uno 5. Báhne verjobieden, háufig alle brei 2(rten, aber burdh

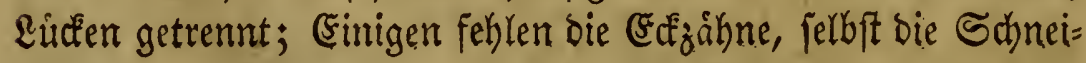
bejaikne im Unterfiefer, aber die bez SDerétiefers bann fehr großs. Dahin:

Die હ叫eingattung (Sus). Dier Zeben an allen Júfen, Die dukeren Eleiner, nad binten geriकtet und erbaben. Zllle 3agn: 
Erfte Rlaffe. Eäugethiete. Bielhufer. Flofienfübler. 29

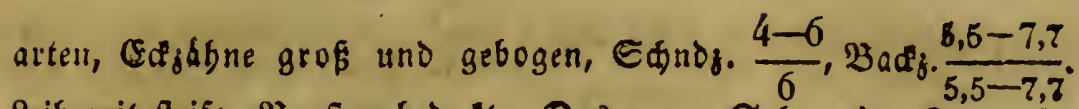
Reib mit fteifen Borfen bededt. Das gem. $\mathcal{S}_{\text {d }}$ wein (S. scrofa)

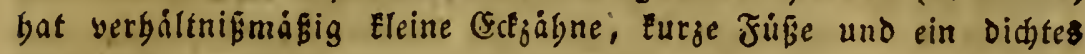

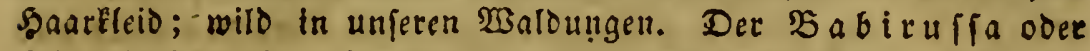

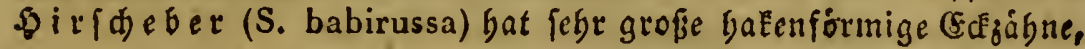

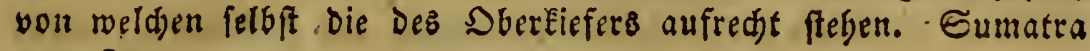
uni Sava.

Das Frufpfero (Hippopotamus amphibius) Gat 4 gleide

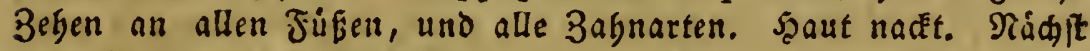
Dem Elephanten bas grógte Eandetjier. Hegypten und alfrila úber. gaupt, in J̧úfie:t. Jrift Pfanzenftoffe.

Die Masborngattung (Rhinocerus) bat 3 Beben an allen

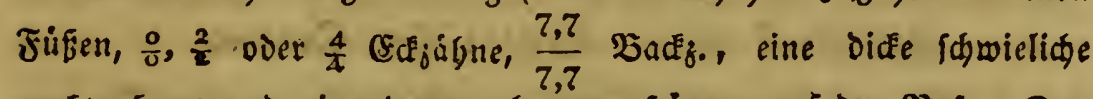
nacte Şaut und ein ober 2 gebogene şirner auf der gtafe. Das a friEa n. $\Re$. (Rh. afrieanus) bat keine Sducidesăbue uno $2 \Re a$. fentorner, Das von $\mathfrak{S}_{\mathrm{m}} \mathrm{m}$ atra (Rh. sumatrensis) ebenfallz, aber yier Sdneidezånne; die anderen beiden oftindifden 2lrten baben nur ein safenforn.

Oer Tapir (Tapirus americanus) bat yorn yier, binten

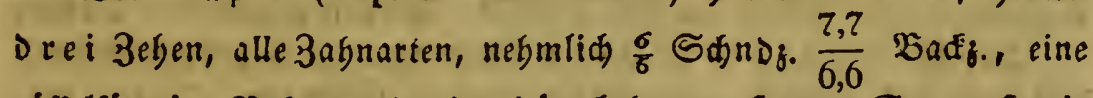

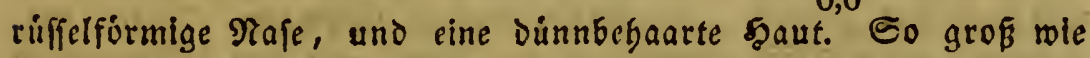

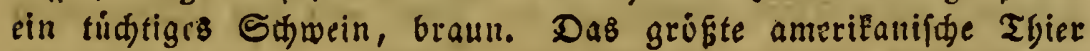
Diefer Bruppe.

Die Elephantengattuni (Elephas) Gat Eeine Edjagne,

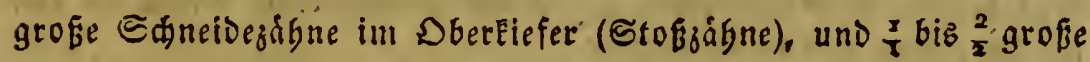

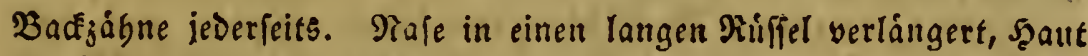
Der zarten nadft, 5 Beben an allen Jüüen. Der oft indifde e. (E. indicus) bat Eleinere Ohren uno Sacţăgane mit parallelen

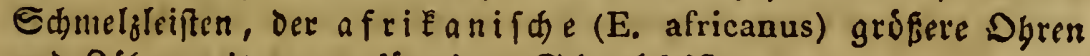
und Bágne mit rautenfórmigen Edjuclgleiften. -

\section{Elfte Familie. Floffenfú}

§. 38. F̧inf Behen an allen grusen, aber burch eine Derbe Scaut jur Flofle verwachjen. Nágel-vorbamben, Eral= lenartig. Zake 3abnarten. Seib behaart.

Die See gunde (Phocae) baben gang bas Gebíz der praubs

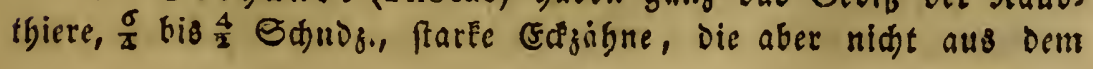


Munde bevorragen und $\frac{6,6-5,3}{6,6-5,5}$ balige Badigange. - Der ges meine S. (Ph. vitulina) ift gelogran, auf Dem Diúcéen bráunlid uno hat feine Shrmufter. Sft uni Siordfee, frift Jifde. Der Seelo we (Ph. jubata) bat eine lange Mábne um Dell Şals und fleine Shrnitifheln. Farbe getboraun. Slitjee.

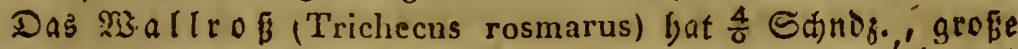

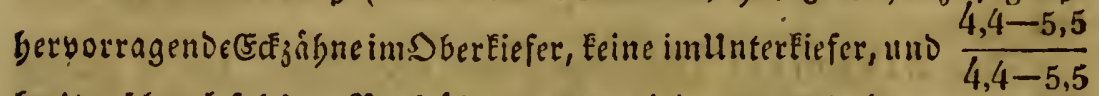

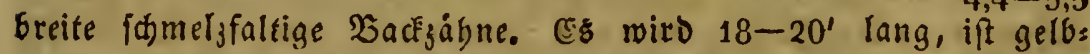
braun, lebt im (Eismeer beerdenweis uno frigt befonders Meerges wådfe. -

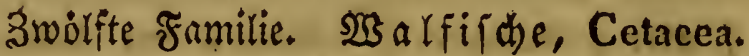

§. 39. Rörper fiīförmig, mit floflenartigen Sorbers glicomaßen und gar Éeinen hinteren. Shrmujher fehlt. Seib nadt, haailos. 2ure leben im Meere.

A. Die einen, melde man Eitenen nernt, baben nads

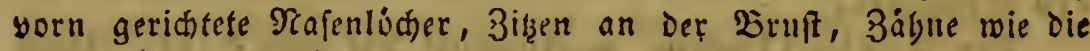

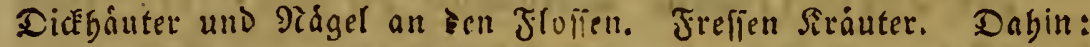

Die Seciungfer (Halicure Dugong), mit 2 grofen Edneis Degábnen und fladem, balbmondformigem ๔dimanz. 7-8' lang. Eibjee. Dies Thier faeint 'bu ber Fabel yon ben Secjungfern oder Eirenen $\mathfrak{2} e r a n t a f i$ ung gegeben buf baben.

B. Die a nderen, oder Deiphine, baben oben auf Dem

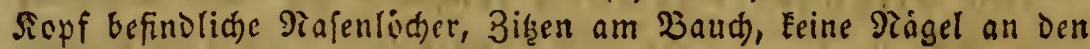
Gloffen, aber Bályne im Siefer.

Der eigentlide Delpgin (Delphinus delphis) bat viele

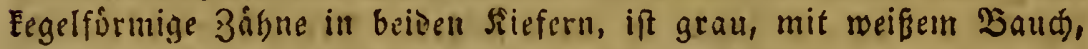
wiro 8-10' lang unt lebt an europáiften siúftert.

Der Pottfifd ('Thyseter macrocephalus). hat nur im Unters

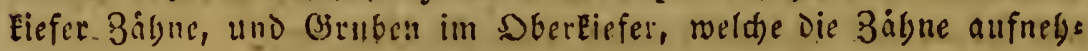
men. Scin תopf iff gref, fein Reib $50^{\prime}$ lang. Eis̀meer.

Der giat wal oder (Singernfifa) (Monodon monocerus) bat nur jwei grofie grade gomundene Etof̧zigne im Doerkiefer, von welden einer immer ausfállt. Reib getblid, grau geffectit. 18-20' lang. Plordineer.

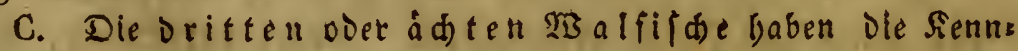
zelden Der yorigen, aber Ecine Zälne, fontern am giande gefaferte

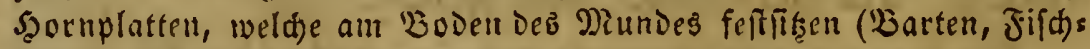
bein). Freffen, mie Die vorigen, thierifwe giabrung.

Der grónládifde 23 a Iffifd) (Balaena mysticetus) bat 


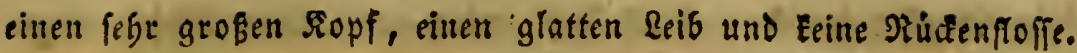
Biegen $50^{\prime}$ lang.

Des Ji in IIfi fí (Balaenoptera boops) Gat einen fämádtigern,

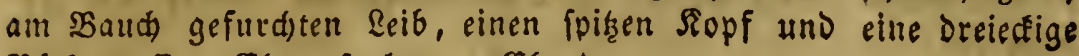
siüdenfloîe. Eben fo lang. Eegenda.

\section{3 weite silaffe. $\mathfrak{S} \dot{\mathfrak{j}} \mathfrak{g} \mathfrak{e} \mathfrak{l}, \mathbf{A} \mathbf{v}$ es.}

§. 40. Seine Sălyne in ben nackten, von Scomfubftang

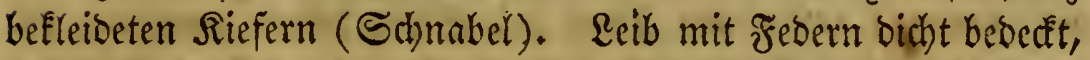
ebentio die zull Flingel umgeftalteten Soroergliebmaß̈en.

Unterweitige (Figenthimlicheeiten ber \$Jogel fino: bie Soneumatigitit ber Sinochen, ס. h. fie fint hohl, leer, uno ibre Scoblumgen fteken burch Sianále in Şerbintoung; in bieje oringt bie \&uft von ber \&unge auts. Dả Bruffbein hat ei= nen Ramm, Der nur bem Straús uno Sajuar fehlt; baz

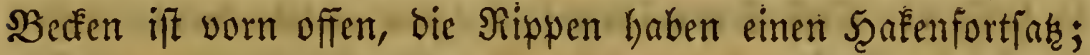

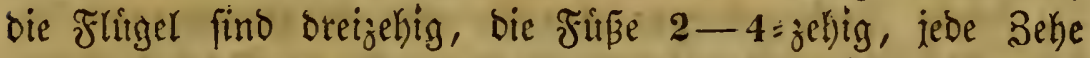

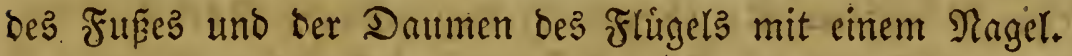

Die Febera find hornige (Sienáchle, weldhe folange fie wachjen, Durch Blutgefápe ernilgrt werbent. Şebe beftelyt aus bem. Sch aft unb ber an beiben Seiten beffelben angefingten Fahne, bas untere hohle Ente bes Sahaftes heiśt Spule, und bie barin befindliche aus Bellen beftekente faltige Scaut nennt man Seele. Sin biejer fteigen bie Blutgefá̄ie binauf. Seber Sogel hat 2 2riten von Febern; Deaffedern, welthe

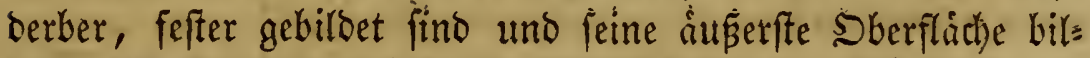
ben, und Flaumenfedern ober Daunen, zartere, weiche, wollige Febern, bie ben untern Raum gwifhen ben Decketedern ausfutlen. Die grofen Federn, welche an ben Selyen ber Sor=

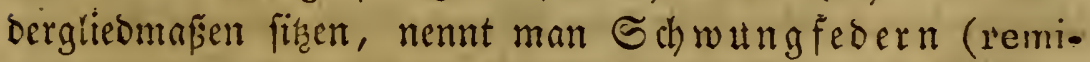

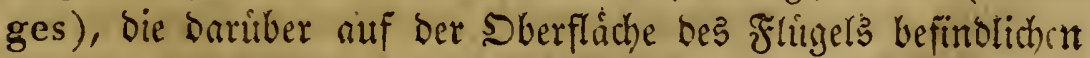
heisen ganj befonters Dedfebern (tectrices), bie gropen

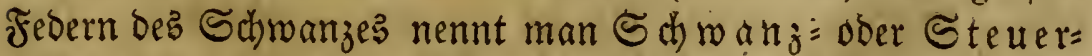
febern (rectrices). Die Federn fino ftets fettig uno laffen 


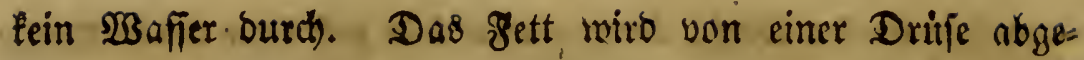
jonoert, bie oben auf bem Shwwanz liegt, unb $B$ úrzels

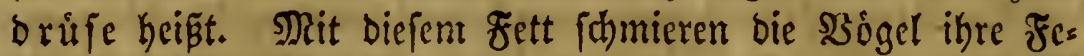
bern ein. -

Der Shnabel uno bie Füpe finto bie einzigen nadfen aheile bes \$ogels. Senen befleibet vorn eine hornige Stheide,

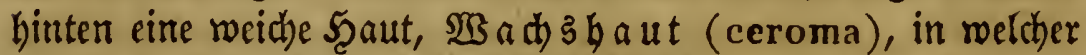
bie Najenlodber liegen. Die Fuse fino von einer mit burni= gen Stuppen befleibeten Scrut überzogen, melche bei ben

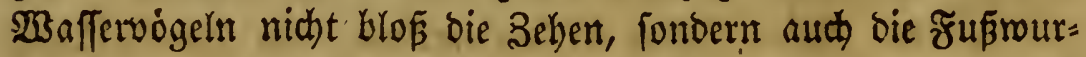
zel (tarsus) uno cinen Iheil bes Schienbeinés umgiebt; bei ben anbern bie Sehen uno Fuprwurzel allein. Jene Billoung Der Füfe bejeichnet man mit $\mathscr{W}$ a obeinen (pedes vadentes), oder, wenn bie Seine fehr lang, mit Stelzenfu仿 (pes grallarius); fehlt fold)en Furen bie Şintergche, fo pflegt man fie $\mathfrak{l} a \mathfrak{u} f \hat{u} \tilde{\beta} \mathfrak{e}$ (pedes cursorii) ju nennen. Sinb alle Sehen mit Scautlappen verjehen, ober ourd) Şaut verbunten,

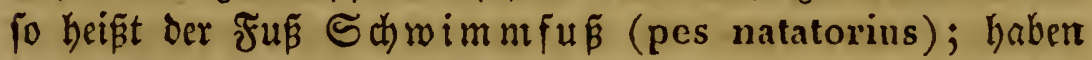
bie Seben farke gebogene firallen und cin georungenes $2 \mathfrak{n}=$ fehn, fo beift er $\mathfrak{R}$ a u b fu $\mathfrak{3}$ (p. raptorius); ift ber Fus flei= ner, zarter und bie Srallen fumpf, fo heist er (3)angfun (pes ambulatorius); fino bei biejen füben bie beiben áußer= ften Beben vermachien, fo nemt man fie $\widehat{S} d$ reitfúpe (pedes gressorii); feben 2 3eben nad vorn, 2 nach hinten, fo mennt man ifn $\mathfrak{A l}$ letterfü (pes scansorius); ift bie ál Berfte 3ehe beroglich, fo bafi fie bald nach vorn, bald nach binten gerichtet werben Eann, fo beist fie 25 enbezebe (digitus versatilis).

Son ben inneren Sorganten iff nur hinfichtrich bes Ma: genక amjufithren, baß̄ er bei allen Siorner freflenben Sobgeln mit 2 bicken balbêgelformigen Slubfeln verieken, bei ben. ubrigen bautig ift. Nor bem sagen finbet fich cin $\Omega$ ropf. Zlle Sojgel legen betanntlich hartichaalige (5ier, 'weldbe

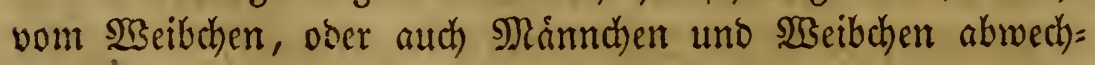
jelno, bebrutet werben, bis bie Jungen aušriechen. Inక auşgebrưtete J̃unge bleibt cann im Reft, uno wiro von ben 2 el= 
tern crmábrt, Refthoder, ober es verlápit fogleich nach ber

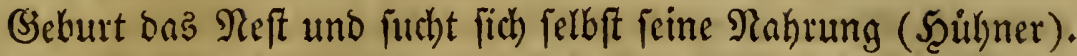

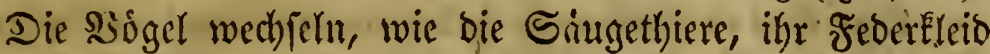
(maufern), uno jwar entweder cinmal im Scerbjt (Şerbjt: mauler), ober jweimal, int Fruifjalfr uno Syerbft. Int biefem Fall iff ibr STeib baufifig verichieden gezeid)net, bas jwifhen Frubjabr und Serbft nennt man Sommer = oder Şoch=

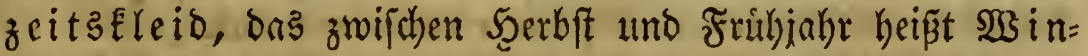
teréleid. -

Die meiffen SBogel unferer Brgent fint 3 ugvogel, o. $\mathfrak{k}$. fie verwechjeln ju gewiffen Salhreszeiten ifren 2(ufenthaltsort mit einem andern; bies gejobiegt im Scerbft nad) Der Maujer

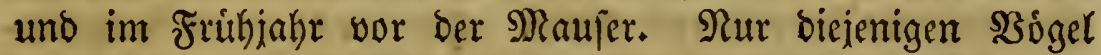
fino einheimifche, welche bei uns Refter bauen und briten.

Man Fennt gegen 5000 verfobiedene \$ojgelarten, weldhe in 3 Soromungen uno adht Familien eingetheilt werben, wie folgt: A. $\mathfrak{a} u f t u \dot{g} g \mathfrak{l}$ (Aves aëreae). Mit furzen wenigftenz bis zum $\mathfrak{L} a u f$ befieberten Fünen, bie fie im Fluge gegen bie Bruft folagen. Samgen Refthucker.

Die $\Re a u b u f g e l$, die Singuogel und dic Spechte.

B. Erovogel (Aves terrestres). Finße gewóknlid) bis jum Enuf befiedert, aber berber und plumper gebauet, siager groß uno breit. Sungen meiftens Eeine Refthoder.

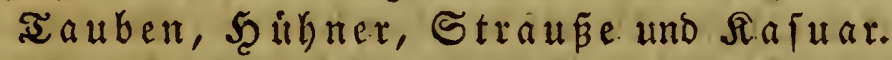

C. $\mathfrak{W}$ affervógel (Aves aquaticae). Füpe biß halb auff Schienbein befiebert, beim Fluge binten ausgeftredt. Sun= gen ber Seiften verlaffen bas Reft logleid).

a. Shne Sdyimmfípe. Sumpfoigel. .

Sranide, Reiber uno Stórde; Sthepfen, $\mathfrak{B a}$ afferhather.

b. Mit Sdhinmfuken. Schimmoigel.

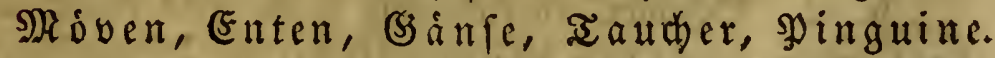

Erfte Familie. $\Re a \mathfrak{a b}$ og $g$ l, Accipitrinae.

§. 41. Schnabel side uno ftark, aber kúrzer als ber Sopf, mit horniger, übergebogener, hafkiger Spize uno brei=

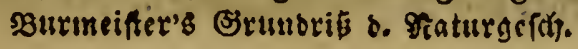




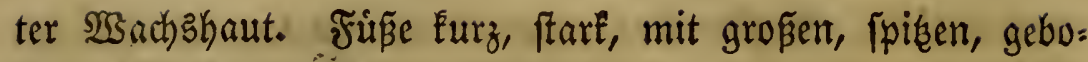
genen Rrallen. Freflen Fleifd).

Die (b) ier (Vultures) baben nadten Siopf und Şals, am Girunde ausgefdmeiften Gduabel und Eurje, Dicke Sirallen. Der gemeine Beier (V. fulvus) ift rotgbraun mit meiflichem, yon Daunen bedectem Siopf und Sals. - Der (j) eier ínig (Cathartes papa) bat Durdgebellde Tafenlodier, Şautlappen auf bem Sdna: bel, ift braun, mit weiBem siopf, uno gelb uno rotb gefäbtem Sđnabel. Eridamerifa. Die Ģeier freffen nur 2las.

Die F alEen (Falcones) baben befieberten Siopf und Şals, feits wárts ftebende 2lugen, ftarke firallen und niemals befiederte 3ehen.

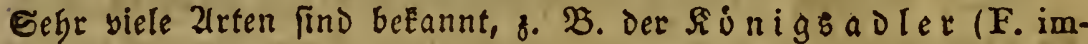

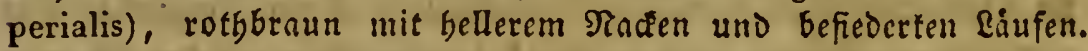
Der Seeader (F. albicilla), braun mit weiflidem fiopf und Sdrwang, und unbefiederten Laufen. Der Ş a bidat (F. palumbarius), oben Dunêtbraun, unten weiß, mit vielen feinen, fomarzen Suermellenlinien, 1 $\frac{\pi}{2}$ bod. - Der Sperber (F. nisus), ebenfo, aber nur $2_{2}^{3 \prime}$ bod. Seide mit gelben Beinen uno $23 a ф b b a u t$, aber blauem Sdnabel. - Det Thurmfale (F. tinnunculus), Ed)nabel mit einem 2ubfdnitt binter ber Spike, Farbe rothbraun mit fdwargen Duerwellen, Edman; mit fdwarger binbe von bee Epite. Brofipe Des Eperbers. - Der EdelfalE (F。 islandicus s. F. Gyrfalco), Sanabel des Borigen, 3eben lebt lang, Eajufe

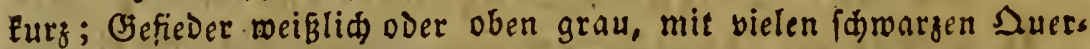
binden und Eángsfleçen, Die im Zlter abnelgmen. Norden (suropas.

Die (E)len (Striges) baben nad vorn geridtte Zlugen, Dic son einem Federkrante (Sdleier) umgeben find, Ednabel und Fuffe Eleiner, leţtere bis auf bie Beben befiedert. Der $\mathfrak{u}$ h u (Strix bubo) Gat Sgrbufdor, ift braun mit fdmargen freuzflecten uno miro $2^{\prime}$ bod. Die Sdleiereule (St. flammea) bat cinen fefr grofen Sdleier, Eeine Shrbúfhel; unten gelb, auf Dem Ritcien rotb. braun, mit vielen sängslinien abwedfelnd weiker und fdrmarges

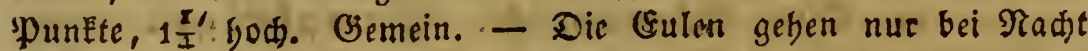
auf Dẹ Raub aus und balten fid am Tage verftect:t.

Smeite Familie. Singuigel, Passerinae.

\$.42. Schnabel allermeifters' Elein, ftets firrjer als ber Sopf, grabe, kegel= ober pfriemenformig; nur (S)angfüre, nadete Buirgeldruife und 12 Schwanzfedern. Zahe baben einte laute Stimme, bie Durch einen eigenen 2epparat am unteren Enbe ber Euftroble (unterem Serblfopf) verftärt wiro; viele fingen melo=

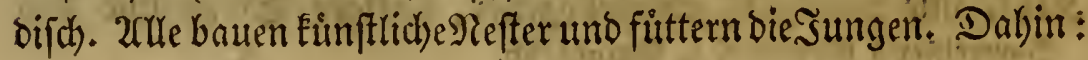




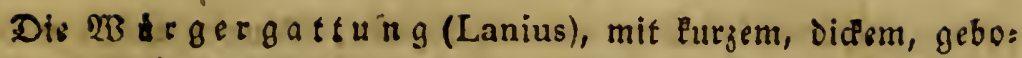
genem, mit einem Seitenjalyn vor Der Spişe verfelyenem Sdinabel. Die Irten frefien junge $\mathfrak{B o ́ g e l .}$ Der $\mathfrak{N e}$ un $\mathrm{t}$ o o t er (L. collurio) ift oben jimmtbraun, unten und am Sopf graul. Epiést Siafer an Dorner.

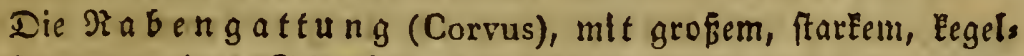

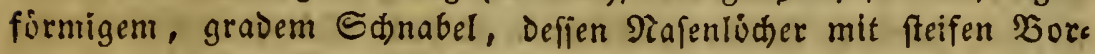

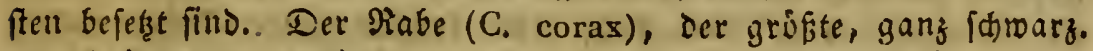
Die Firáge (C. cornix), grau, mit fatwargem Sopf, Jligeln uno Sdmang. : Die. Doble (C. monedula), fidwarb, mit Dickem, grau= lidjem fiopf. u. a. m.

Der Sta ar (Sturnus vulgaris), mit Eleinerem, zarterem Sd)nabel, Defien. Miundmintel fege ftark nad) unten gezogen ift; Befieder blaufdrwarz, meif geiprenEelt. Zuf నiegmeiden.

Die Droffe In, (Turdus) baben einen Elirgeren, graden, ete

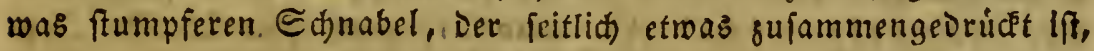

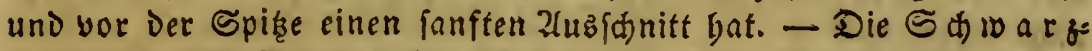

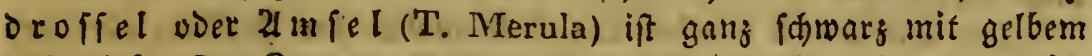

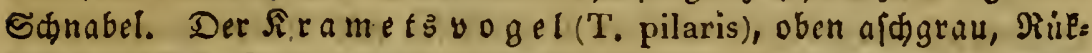

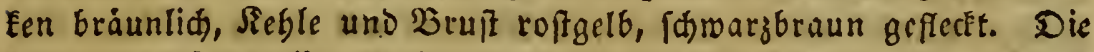
Singoroffel (T. musicus), oben divenfarben, unten gelblic mit Dreiedigen ounkelbraunen Flecken.

Die Sả $\operatorname{ger} g$ a $t$ tung (Sylvia) hat gang bie Form und bas

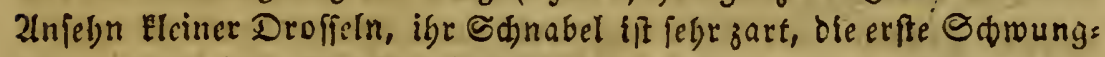
feder febr Elein. Dic $\mathfrak{R}$ a digfigll (S. luscinia), rotbbraun, am

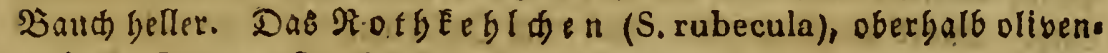
farben, Jieble roftrotb; u. y. a.

Die "'o a fit $\mathrm{e} / \mathrm{f} \mathrm{e}$ (Motacilla) bat einen etwas lángeren aber feineren, fipitzeren Shnabel uno einen viel längeren Sdwang. Die weifi $\mathfrak{b}$. (M. alba), oben grau, Stirn, baud uno de auferen

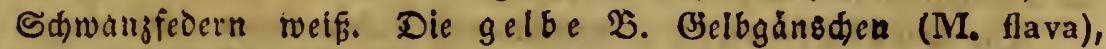
oben fommukgig grin, unten gelb, Siopf aldgrau.

Der 3 a unE unig (Troglodytes parvulus), Gat ben Éanabel Der 2origen, Dod iff.er fanft gebogen, und Der Єdwang fegr eur Geficoer braun, finmarz punEtirt.

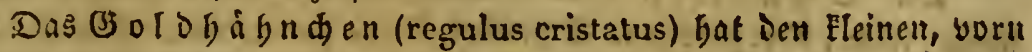
zufammengeirúdten, aber graden Sdnnabel bes Dorigen, und ein rundes yon einer Feder bededtes Nafenlod; ; Befieder olivenfarben,

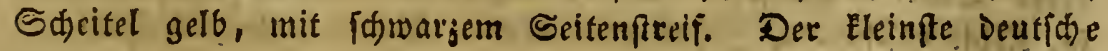
2ogel.

Die Meifengattung (Parus) Gat einten febr Eleinen, Eurrjeren, aber aud' Dideren, mebr Eegelformigen ङd)nabel uno eine mit meb)

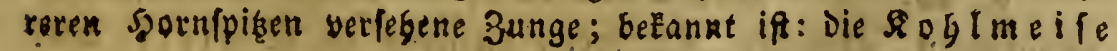


(P. major) obett fơnarg, unten gelbgrůn, Wangen weif; uno Die Beutel mé ife (P. pendulinus), roftroth, Baud gelblid, Stirn

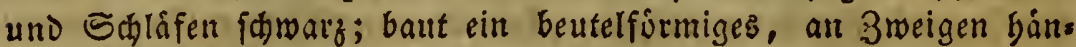
gender Seft. -

Die \&erchen (Alaudae) Gaben einen ctraz grofieren, ftökeren, Eegelformigen Sdnabel und einen felbr langen Sporn an Der \$in= terjebe; Farbe gelbgrau, 'mit braunén Fieden. Die Ş a (A. cristata) bat auf Dem fiopf eimen langen, fpigen FeDerbufd.

Die Finken (Fringillae) baben Die ftireften, dickften, bärte: ften, aber babei nidht lingften ङdhabel unter allen Singuigeln. 2lü freffen fie nur Samereien, Die Säuger und ifre Bermanden Dagegen Snfelten. Der Sper!ing (F. domestica), grau, Rile Een braun und fdmarz geflectit, Jegle bes Jiannchens idwarg. Der $\mathfrak{D} u$ ch fine (Fr. coelebs), oben Eaftanienbraun, unten beim

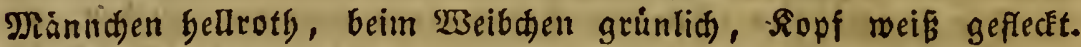
Der I a narienvogel (Fr, canaria), ganz forbefelgelb. Sebt nidit melye rild.

Die $\mathfrak{A}$ mmern (Emberizac). Sollabel Der. Finen, aber ber DberÉefer ausgebogen und Der Unterliefer mit cinem bervorfprin= genden Minkel an ber Mundecke, Baumen mit einem ŞDicker. Die

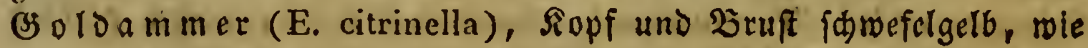
Der graue Riucéen braun geflectit.

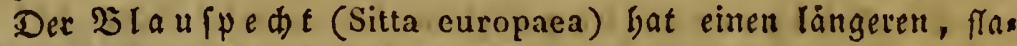
deren, graven, alm Ende abgerundeten Ednabel, und einen tút: zeren, breiten, runben Sdmanz; Jarbe oben blaugrau, unten fleifd: rotb. Şüpft an Baumftimmen nad) Snfeften.

Der $\mathfrak{B}$ a $u$ mla $\mathfrak{a} \mathfrak{f} \mathfrak{e} \mathfrak{r}$ (Certhia familiaris) bat einen buntren, gebogenen Sdnabel, lange strallen uno fteife, fpibe Sdwanzfedern, Farbe braungriu, weif gefprentelt. उeitig im Fribiabr in (Jirten, an Baumftinmen Gipfend, gutraulia.

Die'Sconiglánger (Nectarineac) baben einen nod lánges ren, feineren, fanft gebogenen Sdnabel uno eine lange, róbrige, fum Sconiglaugen tauglidhe 3unge. Dicle ?rten, alle prád)tig gold: oder exffarben, meift aus 2lfrika; gelten fur Stolibri's.

Die $S_{\text {d }}$ malben (Hirundines) baben lange Flígel, Eleine,

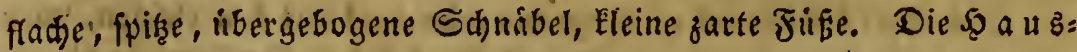

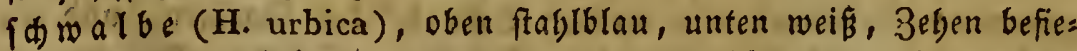

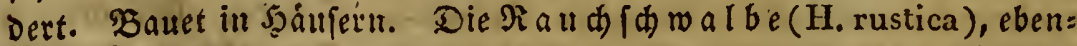
fo, Siegle roftfarben, 3cben unbefiedert. Bauet auben an Şäufern.

Dritte Framilie. Spechtooggel, Picariae.

§. 43.: Mit groß̧en ben Sopf an Lange übertreffenben Sdbnabeln, gróferen, ftórferen, meiftens zu Sdreit = ober 
Slet terfinßen umgebildeten ßBeinen und befiederter Bürzel=

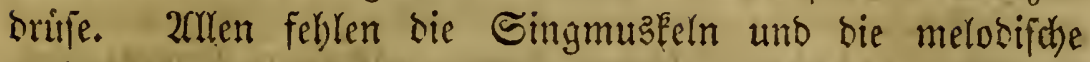
Stimme. Die wenigften B̧tieder Diefer infaltšreichen (Sruppe firo in Guropa zu Scaufe, bie meiften leben zwifthen ben Sizenoefreifen. Merfwirsig finto bejunbers folgente:

Die $\mathfrak{S}_{\text {ol }}$ ibrigattung (Trochilus), melde Die Eleinften aller

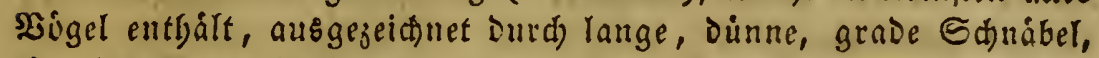
cine lange, runde Zunge, melde ibnen gum (Einfaugen Des Bli:= thentgonigs Dient, uno zicmlich lange, bismeilen yorn bornige Schmungfedern. Sie leben nur in 2 (merifa uno baben ein fdinnes glänjéndes Bsefieder; mande Ztrten miegen nur einige Bran; die cier mie Exbfer, abor lánglidj.

Der $\mathfrak{R a u e r f e g l e r ~ ( C y p s e l u s ~ a p u s ) ~ b a t ~ g a n g ~ D a s ~ 2 l n f e g n ~}$

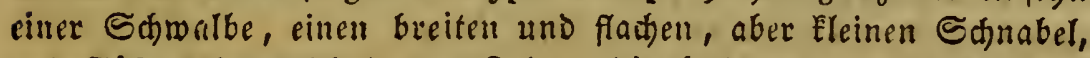

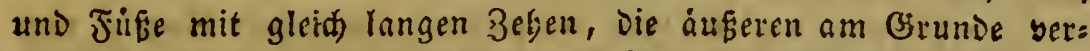

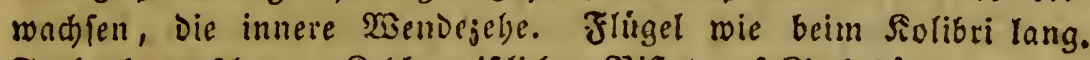

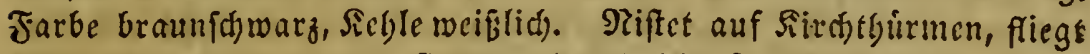
fdaarenneis mit lautem Oefderci Durd Die Etrafien.

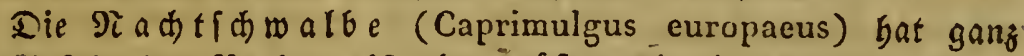
Das Itnfern Des Sorigen, ift aber gruffer, wie eine Droffel, bat, ei= nen febr brciten, aber Eurzen Ednabel, Der bis Ginter Das zluge gefpalten ift, und Durdi Şaut yerbundene Beben. Nagel Der Mit: telgebe am inneren Piande gefámmt; Gefieder Düfrer, braungrau

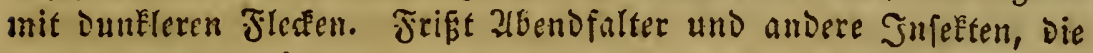
$\mathfrak{e r}$ im 3rvielidft fíngt.

Der (Eibvogel (Halcedo ispida) bat einen grofen, graden,

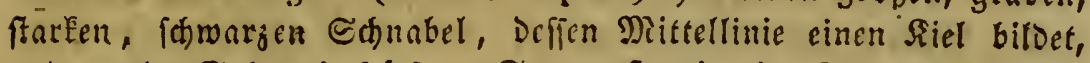
uno rothe Sditeitfífe. So grof́s wie ein Dompfaffe, oben

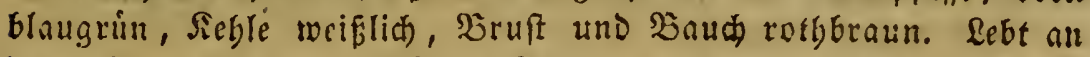
Den lifern won Een, Flififu, Teiden, wo er aud niffet.

Die IRandelEr ábe pDer Blauracfe (Coracias garrula) bat ganj Das 2lnfegn eines Erábenartigen 20 gets, einen graben an Der Epite ibergebogenen, fahmarjen Edhnabel, uno getbe ziemlid zarte

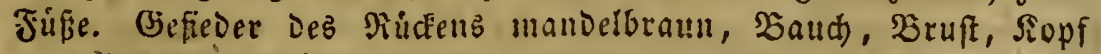

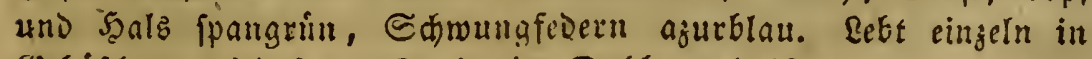
Bebiffden; mird to grof wie einte Doble, und ift wogl Der fajonfte aller beutifen Digel. Frift JufeEten.

Sie Rasbornug gel oder fialaoz (Buceros) baben grofie,

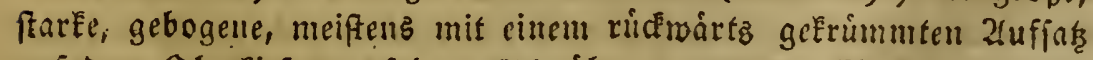
auf Dem Dberkiefer verfergene Sduáber, uno Diăe, Eloģige Sd)reit= füfer. Cie fino meiftens grofie Bögel, mit fømar

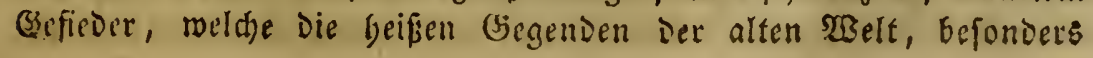




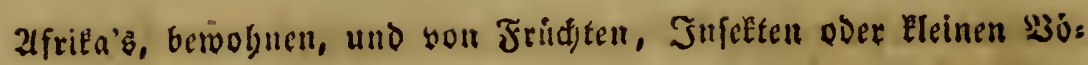
gelu leben.

Der 23 iebegouf (Upupa epops), mit einem langen, dún: nen, fanft aebogenen Sanabel, und einer aus einer Doppciten

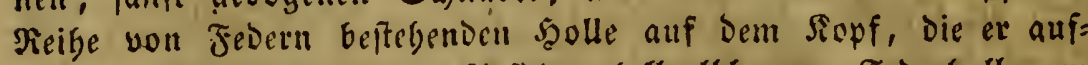
uno niederfhlagen Eann. Sisficoer bellgelbbraun, Federgolle am Ranie fdwarb. Fligel fhwarz, mit weifen Querbinden. In 2Beidengebuifden.

Der SiufuÉ (Cuculus canorus) Gat Die Grófe Der Doble, eia nen leidht gebogenen Eduabel mit ganz rundem Tafenlod, und Siletterfífe. Der Edwanz, beicnders mand)er auslándifaen 2trten,. ift lang, das Geficoer grau oder braun, mit dunflen Suerlinien.

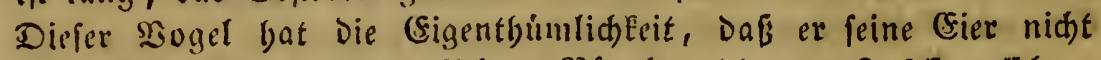
frlbit ausbrittet, fondern Eleinen 2 ögeln, Die yon SnfeEten lében,

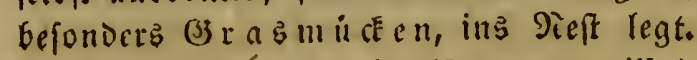

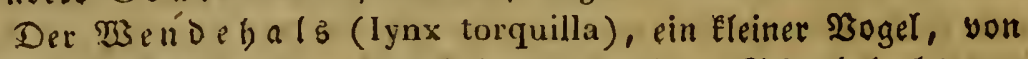
Der (srobe einer Eerde, mit fleinem, graben Sdnabel, langer, weit ausfrecfbarer 3 unge, Siletterfificu und grauem, Didbt mit Elei= nen fibwarjen Drricten befteutrm Befieder. Lebt paarweis uno niftet auf alten $B$ anmftummen.

Die Sfattung Der $S_{p e d} t e$ (Picus) zeichnet fid aus ourdh cinen grofen, graben, febr ftareen Eduabel mit lingliden Nafen: lichern; ourd) eine lange, runde, weit ausftred bare 3unge; Durd sletterfíbe und einen ffikzen, fteifen Sibranz zum Znftemmen. Die Spedte leten in Den Sisildern, und Elopfen an Die Baumftám: me, um Gnfekten auz Den Ditzen uno Spalten Derfelben Gervorzu= treiben. Der $S$ d wa $\mathrm{r}_{j}\{\mathrm{ped} t$ (P. martius) ift fo grof mie eine

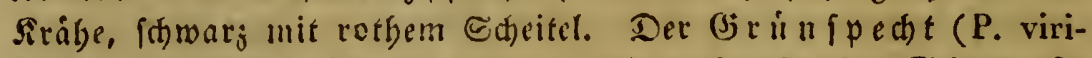
dis) ift fomuszig grun, mit formarz und meif geftreiften Sdmungfe: Dern uno rothern ङheitel.

Lie Pfefferfreffer voer $\mathfrak{T} u \mathbb{E}$ a \& (Rhamphastus) baben die grifiten Gdnabel von allen, Die wobl $\vec{j}$-4mal fo lang fino als bev Siopf, bice, brcit, funft getogen, gegen Daz Ende zufammen:

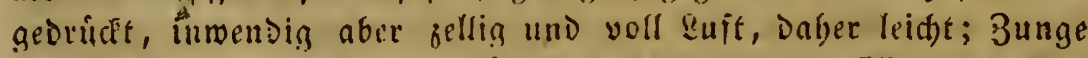
feDerformig, bornig; Ifletterfúfe. Sie liben in Brafilicn und fref:

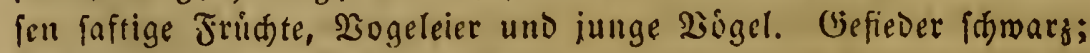
theiluciz gelb, meif over rutf).

Die Papageien (Psittaci) endid baben Eurgere aber febr

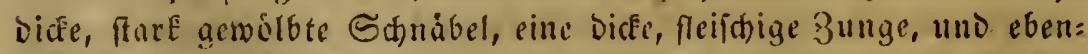
falts Silenterfífe. Sie freffen aud fleifdige Jrinte. Die $\mathfrak{K}$ a $\mathfrak{a}=$ D u s babcu befiederte Bangen und eille grofe Federbolle anf Dem Sopf, Die fie aufridten und nieverlegen tónncu, $\mathfrak{z}$. \$. Ps. sulphu-

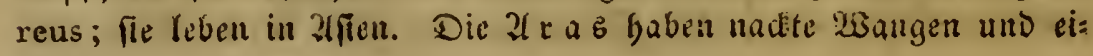




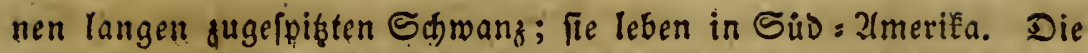

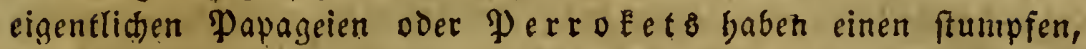
breiten Stawanz, und bald $n$ a fe, twie Der graue p. (Ps. eri-

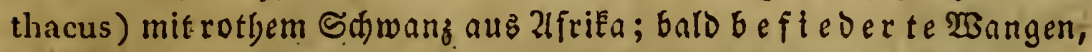
wie Der grúne p. (Ps. ochrocephalus) mit blauer Stirn und rotgem Flea am Jlügel und Sdmanz; aus : Braftitien.

\section{Sierte Fantilie. $\mathfrak{a} \mathfrak{a} \mathfrak{b} \mathfrak{E} \mathfrak{n}$, Columbinae.}

§. 44. STeine, vorn hornige, binten båutige Schnobel

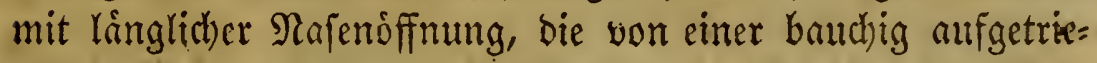
benen Schuppe bebeclt ift. Sileine, Eurze (Sangfúse mit klei= nen abgeftulsten Någeln. SBauen auf Baumen ein Eumftiofes Teft uno futtern ihre Stmgen. Freffen Rorner.

Die Battung oer $\mathfrak{T}$ a uben (Columba) ift liber bie gange Eroe verbreitet, befonders aber in Den wármeren \$limaten zu Şaule. Bei uns giebt es Drei lrten; Die grofe Rin gelta ube (C. pa-

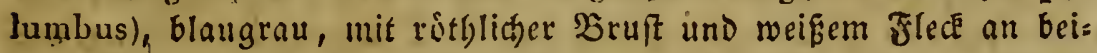

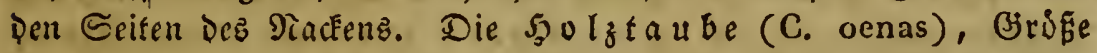

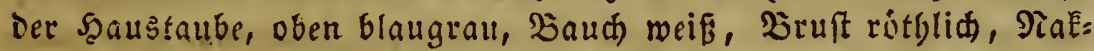
Een bronçefarben. Die $\mathfrak{T} u \mathfrak{r} \mathfrak{t} \mathfrak{l} \mathfrak{t} \mathfrak{a} \mathfrak{u} b \mathfrak{c}$ (C. turtur), feifdfarben, Sheitel und Sinterricken grau, Sdwungfedern braun, Decffedern

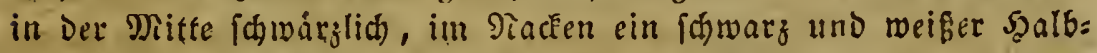

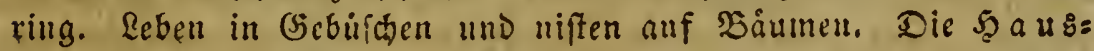
taube frammt von ciner 2lit (C. livia), Die nof wild in Eủd: Curopa mo Miord =? (FriEa yorfommt, fie if bleigrau, auf Den Jligeln mit zwei fatwargen Binden uno fabmarzen Sdmungfebern.

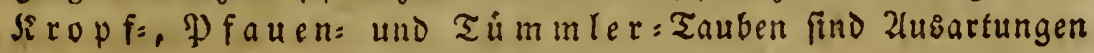
uripringlid, verffiedener, oftindifder inten.

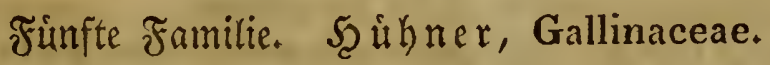

§. 45. Sthnabel Flciner, bider, libergebogen, blow hor nig. Najenlocker verftedft. Füß̈e' ber \$origen, ooer ftärker,

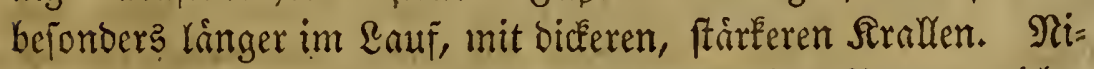
ften faft immer auf ber Erbe unb futtern ibre Sungen nicht. Freffen Fiorner.

Der $\mathfrak{Z}$ uer $\mathfrak{b}$ a $\mathfrak{n} n$ (Tetrao urogallus) bat einen naditen, war: jigen Streif über Dem 2(uge, befiederte Lauffe, aber Eeinen Sporn;

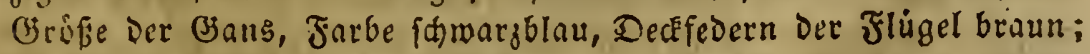
MSeibden risthlidbraun, am SBand) graulid, úberall føwar geflectet.

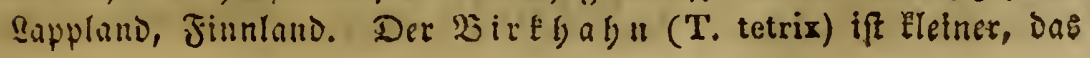




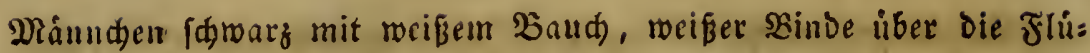
gel und gabeligem Єdwanz; Das Wiseibden beller roftroth mif gelbli= Gen JeDerrandern und tief auzgebudtetem Sdmang. 2(ud) in Deutidland.

Das $\mathfrak{A} \in$ b $\mathfrak{G}$ u $\mathfrak{h} \mathfrak{n}$ (Perdix cinerea) bat einen iberall befiederten Siopf, aber nadite Iáufe; erfte Sdjwungfeder Érizer. Seine Tarbe ift graubraun, auf Dem Múçen geiprenfelt, Sichle Des Männd)ens roitgedb, Der Daud braun. Oemein in Dentfhland. Die $\mathfrak{B}$ a $d$ ): tel (P. colurnix) iff fleiner, beller graugelb, mit Dunflerem, bell

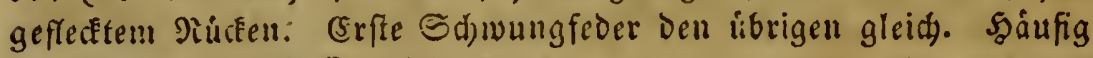
im Sommer auf Siurnfeldern.

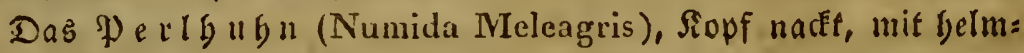
artigem 2luffas, Scautlappen am llntertiefer. Farbe bellgrau, mit

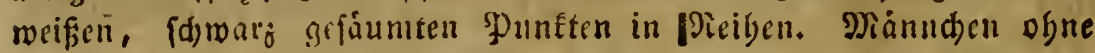
Sporn. Sivero = ?frika.

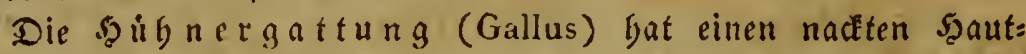
Eamm auf Dem Siopf, Şautlapwen an Der Siegle und Die Dlännden

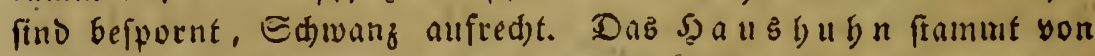
einer 3rt (G. Bankiva), Die fid nod in Dftindien milo findet, wic Die Eaftanienbraumen nit goldgelben Salsfedern verfebenen Sadgne, und wie bie graubraune mit fowárjliden Sucrgeidnungen und gelbgerinierten Şalbfoern verfebene Somne gezcichnet ift.

Dic Fa fa $11 \mathfrak{e}$ (Pliasiani) Gaben nut cinen nacken ring ums Zluge, einen langen fpitzen. S占wanz, und Das Mliunden bat Eurge Sporen. Der ge me ine 5 . (Ph. culchicus), roltfarben mit fdrárz= liden Suetfleften, Sopf metallifh griul Scente grau, braun ge: flectét. Efammt aus Dem wefflid)en Ilîten.

Der I f a u (Pavo cristatus) bat nur nadte Wangen und ei=

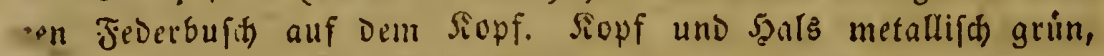
Die Burgelfedern beim Miámchen febr grofi, bronęefarbig, jebe am Ende mit cinem 2lugenflese. Yrito in Jnoien.

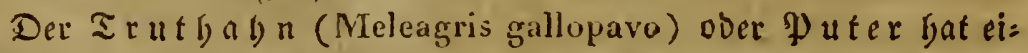
nen nacketen mit vielen Tisarzen uno einem Bipfel auf Der Stirn verfebenen Sopf und 3orich als, fo wie einen rabformig aufricht: baren Edivanz. Sifiedec braun, jede Feder yor. Dem Ende mit fdrwarzen Suetftrid). Jisinnden mit einem Sanarbrifdel an Der Sruft. Stanmt alts gioro=21merifa.

Sedjate Familie. Raufvigel, Currentes.

§. 46. Sieine zum Flicgen taugliche Flügel, Şals ver= longert; Schnubel flach, fo lang als ber Sopĩ; SBeine jebr

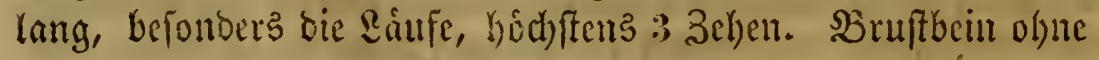




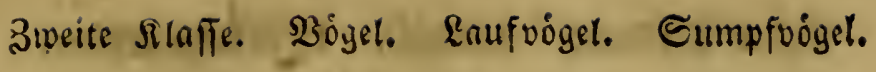

Ramm; Beefen vorn geichlofien. İungen werben nidht ge= fittert. Diefe Sjogel laufen idhell umo fahlagen babei mit Den Flitgcln. Dakjin:

Der Si a fuar (Casuarius galeatus), mit nadén Siopi uno ei:

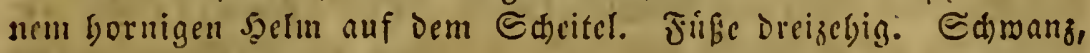
Solf uno Dberyals blau und roth, nuctt. 2uff ๔umatra, Java.

Der a meriéanifde Strauf voer $\operatorname{Tan}$ a u (Rhea ameri-

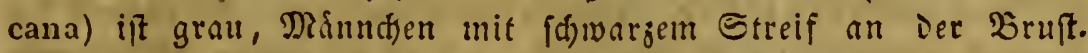

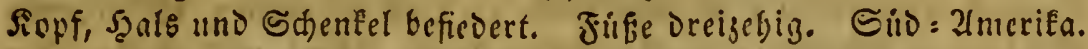

Der eigentlide $\mathcal{S} \operatorname{tra} \mathfrak{u}$ ह (Struthio camelus) bat nackften

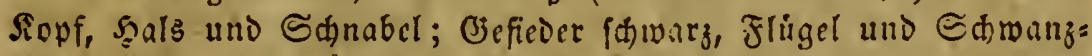
fcocru meif. 3 wei $3 c$ ben. 2f frięa. Cier wie ein Sinderkepf, von mebreren Tibeibden in cin gieft gelegt, worauf fie abmechfelnd briten.

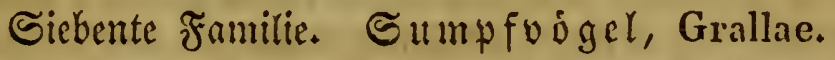

§. 47. Eange SBeine, mit balb befiedertem Echienbein uno meiftenz freien Bchen. Scaţ ebenfalls lang, Der \&ánge ber Beime entipred)eno; Sdhnabel bald firrzer bicfer, bald lánger

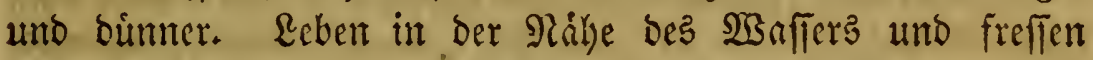
vorzugsweife thierifhe Sahrung. Futtern bie Sungen nicht.

Die $\mathfrak{T}$ appe (Otis tarda) bat einen Eurzen, zien'id breiten

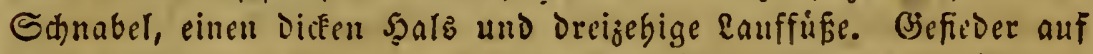
Dem Rúcken gelbbraun mit, fdwarzen Suerftreifen, im úbrigen

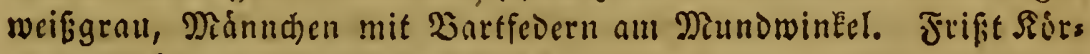
ner. Girófe Der Ganz uno Drúber.

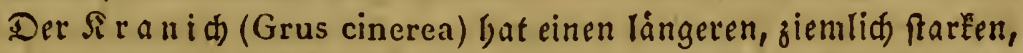

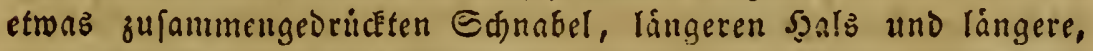
vierzegige beine, Deren Şinterzebe nur mit Der Epite Den booen

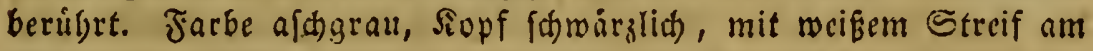

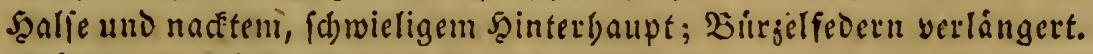
Briffer und biber als Der Etord. Rebt (d)aarenmeis, mandert wie

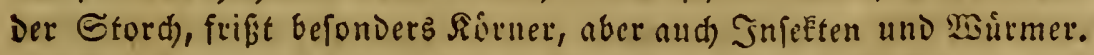

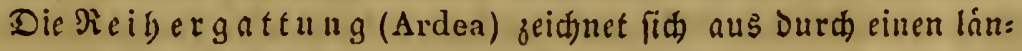
geren, fpitęeren, feineren Sd)nabei, fürzere Lảufe, aber längere, mit

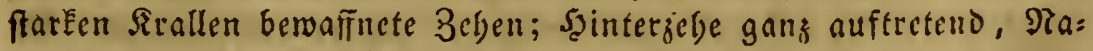
gel der mittleren am áuferen Fande gekerbt. 3mei 2lrten fino bei

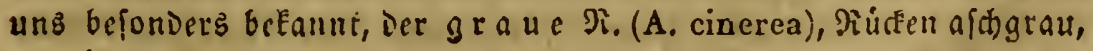

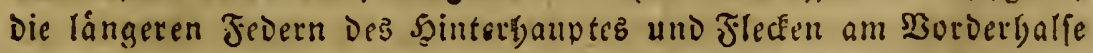

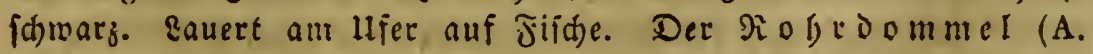

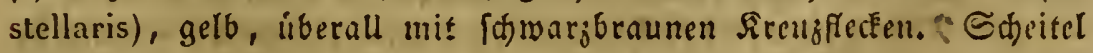

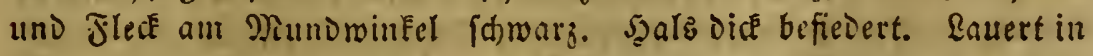
Súmpfen und frift von 3eit zul 3eit einen Dumpfen Ton aus. 
Die Stór he (Ciconiae) baben viel didere, bobere Sđndbel, längere Beine mit viel Eúrzéren 3ehen und ftumpfen Firallen. 2lu: genring, bismeilen fogar ber ganze Sopf, nackt. Der we ifie 5 t. (C. alba) bat rotben Sdnabel uno Jübe, einen fdwarzen Strid) um Das Zuge und idmarje Sdmungfedern. Der fd warze 5 t. (C.

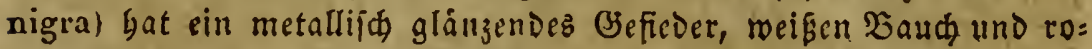
then Ghuabel uno Jifie. Beide freffen 21 mpbibien, befonders Jrifde.

Der $\mathfrak{i}$ jffelrei ber (Platalea leucorodia) hat Die Beftalt Des ఇeigerz, aber einen nach bem Ende zu breit georidéten, loffelfermig ermeiterten Єdnabel, nackte Zlugenringe, Bigel uno sieble. 2lm Sirunde Der aufieren 3ebe eine breite Szautfalte. Befieder meif, Sduabel gelblid, am Rande, wie tie Fifíp, fdwarz. Eauert am Ufer auf Jifhe uno Tisurmer. Củd = Eurapa.

Der Fla mi gen, winEelformig abwärtz gebogenen Eduabel, Den längften Şals und Die lángiten Beine. Beben Eurz, Die Drei vordern Durd $\subseteq$ dmimm: baut verbunien. Gefieder feuer: ober bläroth, Shrwungfedern \{droarz.

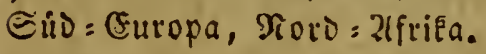

Der $\mathbb{i}$ i $b$ i (Vanellus cristatus) bat einen Eleinen Ednabel mit langer Nafengrube uno vierzebige Jübe, Deren Sainterzebe fegr Elein 1f. Siefieder $\{$ dwarz, erjfarben fdillertio, im Nadfen ein Sdjupf lángerer Seorra. Şinterbals, Druft, Baud weif. Seine roth. 2luf fumpfigen Witefen; frifit InfeEten uno Märmer.

Der $\mathfrak{S} \dot{a} \cdot b$ el $\{d) n a ́ b l e r(R e c u r v i r o s t r a$ avosetta) Gat cinen lana gen, feincn, fpiksen, aufmártsgebogenen હdnabel und Edwimmbáte

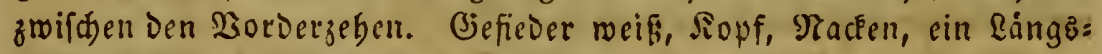
fleck auf ieiem Jiugel, Sdmungfedern, Sd)nabel uno Beine fdrwarz. Siuften Der Torblander.

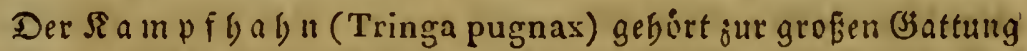
Der Stranoláufer (Tringa), welde an Dem graben, feinen; aber etroas ftumpfen, nidht febr langen, meidhen, in Der Jhitte biegfainen Sdhnabel, und Den langen vierzebigen Beinen Eenutlid ift. Er un= terfdeibet fid Durd fein bunteb, bräunlidgraure, DunEler geflectes Siefieder, und Das Mianndent befonders Durd eine grofie Salserauje und viele Eleine Warjen vor Dell Iugen. Fiffen Der Sfffee.

Der beilige $\mathfrak{J}^{6}$ is (Ibis religiosa) gehort aud) bierber. Er

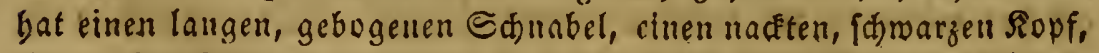
cin meifes bicfieder mit fdwarzen, bufdhigen Dirrzelfevern und nidt febr langen, fobmarzen Beinen. Siurper wie ein Şubn. Lebt in 2le: gupten und waro you Den alten 2legpptiern als gottlid verebrt. -

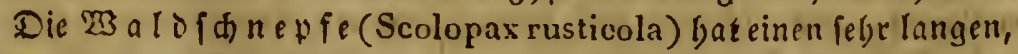
ftaken, grnden Sdnabel, grofie nad binten geridtete Zlugen, Ekirzen \$als und Eurje Beine. Befieber braun, fomarg in Die Duere ge= 


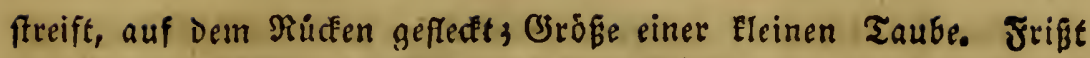
InfeÊten und Tistrmer. Bei uns nur auf Dem Buge.

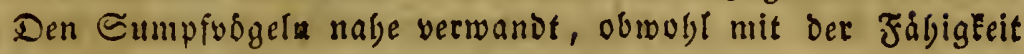
Des Sdimimmens begabt, ift Das $\mathfrak{W}$ a f fer b ub $\mathfrak{n}$ (Fulica atra), Eennt: lid) an Dem Eurzen, feitlid zufammengeorícten, fpiken Єannabel, yon weldem ans fid) eine fommielige Sant auf Die Stirn bin fortfest, an Den Eurgen Jüúpen, und Den langen, jever jeits mit meljeeren abgerun: Deten, gefrangten Rappen verfegenen Zegen. Farbe fdjwarzgrau. Sróke Des Şugns. Sdjaarenweis auf Seen in 'Binnenlande.

2(h)te Familie. Schwimmvigel, Natatores.

§. 48. Durd) hurzere, breitere Sdłnábel, einen Eurrzeren Scats uno kurzere Beine, Deren Zehen burch) Schwimmbäute verbunden, voer mit breiten, feitlichen Scautlappen verfehen find, untericheiben fich biefe sogel von ben vorigen. Sie

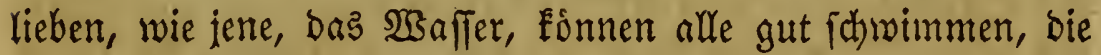
meiften auch tauchen, futtern ihre sungen nidht, bauen funft= lofe ober gar Eeine Refter und freflen theils thierijche, theits pflanjliche Nahrung.

Die Dis yengattung (Larus) bat cinen ftare fufammenges

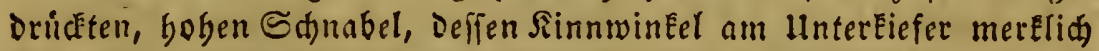
Gervortritt, und meifens vierzehige Júfpe, die Drei vorderen Durd

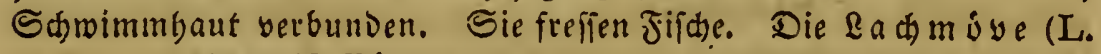
ridibundus) ift wciß́, Piúdén und Deckfebern ber Frígel bellgrau, Sispf braun, Edunbel uno Jüke rotb. Die Jungen grau gefprenfelt. Brof́pe Der firálje.

Das Zlbatros Diomedea oxulans) mit grofem, bafenfirmi: gem હannabel und róbrenfórmig bervortretenden giafenlidern. Beben burd) Sdrwimmbaut verbunden, Die gintere feglt. Befieder weif,

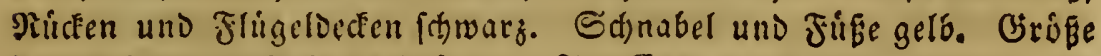

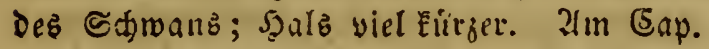

Der Jiegat toogel (Tachypetes aquila) Gat einen fdmáleren, frarfen, GaEigen Sdinabel, nackte Siefle uno Zlugenrano, balbe Edmimmbante zrwifhen allen wier Beben, befiederte Ráufe, einen ga: belfirmigen Sd dranz und fegr lange braun. Etwab griffer als eine Ente, Flingel viel lánger; fliegt an: balteno. Zmifden Den Wende Éreifen.

Der Seerabe (Carbo cormaranus) oder die Gdaarbe, bat die Siennzeiden Dę Sorigen, aber Élirzere Sđiningen, einen abgerundeten

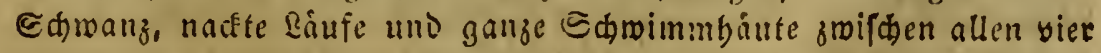

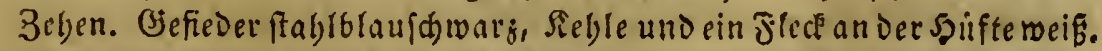
Plordifde Siuften, in risinter aud an beutfden, frifit bejonders 2lale. 


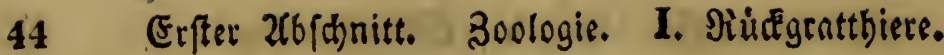

Der Pelie a (Pelecanus onorrotalus), ober Die Siropfgang, bat cincn langen, breiten Sdnabel, an Deffen Unterkiefer cin weiter báutiger Sack bángt; Epitge mit cinem Saaken beroebrt. 2ulle vier 3eben Durd Sdrwimmbăute verbunden. Sefieder feifdrotb, Echmingen fdroarj. Jiuften Des Mrittelmeeres.

Dic Gattung Der (j) ånfe (Anser) zeidnet fíd aus Dutch) ci= nen am Brunde boben, am Rande mit einer geferbten \$̧aut ver: febencn, an Der Spige mit einer Şornfaluppe befeftell Sdnabel; Tinfe vierzebig, Die orei verderen burch Schwimmbant verbunden. Die fabme ${ }^{5}$ a $n$ z ftammt von Der, wie eine ganz graue fabme grfárbten, wilden of ans (A. cinereus), Die uiberall in Deutidhlamd auf Seen und an ben Súften angetroffen mird.

Die d) wa ne (Cygni) baben einen lángeren 5yals, beeitere, fadbe Sdnabel und nackte 3ügel. Der ftumme $S_{\text {d. }}$. (C. olor) bat einen rothen, binten fdotwarjen und mit einem Şicker yerfebes men Shnabel. Der Sing do wa a (C. musicus) bat Eeinen Sojofer und eimen fdwarzen, binten gelben Ednabil. Biefieder bei beiben meip, in Der Jugenb grau.

Die Enteugattung (Anas) unterfiteidet fíd) yon Den Ed)wánell, bei gleidem ban bes Edhnabels und Der Beine, Durd) fúrjeren Sals und volleommen beficberte 3ingel. Frefien, mie bánfe unD Shwoine, am liebten Pfanjennabrung, aber aud Bewirm,

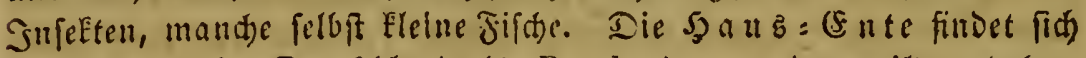
wild liberall in Deutfjland (A. Boschas); yon Den willen baben bie Diánnden (Entrid)e) ganz Die Brópe und Tarbe Der jahmen

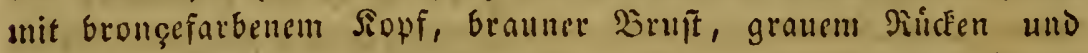
weiflidem Daut ; Die Weibden Dagegen fino rie Die grau, braun uno fdwarg gefprenfelten mit erjfarbenem Epiegel gezeidueten zagmen Beibden gefátut.

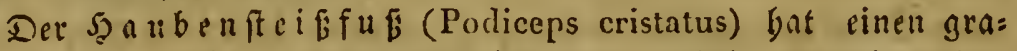
Den, fpisen, etwis zufammengesrickten Ednubel, uno vierzebige, mit Ereiten Saautlappen an ocit Beben verfebene, fratE nach binten seftellte. Jüfe. Gieficoer auf dem Diucken braungrall, Interfeite weif, um Den fiopf eine roftbraune, ftrwarggefaumte FederEraufe. Uriffe Der Ente und Driber, syale uno Bène linger, Zluf inlän= Difden Bemafiern. Frefien Sufekten und Jifde.

Der Pi

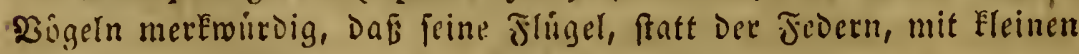
Szornfduppen befleibet fino. Eduabel gicmlid grode, fpiţig, etwas zufammengeorickt; Jilfe ganj binten, viergebig, mit Eurjen brciten Läufen uno ganjer Edwimmbaut zmifhen Den Beben. Man Eennt mebrete 2luten Diefer Battung, Die alle an Der Eublpitze Der Sonti= nente vorkommen. Sie geben aufiedt und fhroimmen mit Dem 


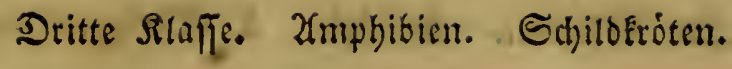

ganjen Reibe im \$isaffer, wobei bie Flügel als flofien Dienen. A.

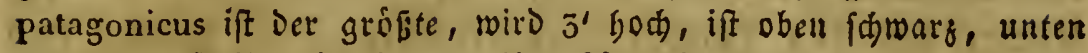
weif́, ałm Şalfe mit einem gelben Rángşftreif.

\section{Dritte $\mathfrak{I} \mathfrak{i}$ ffe. $\mathfrak{A m p h i b i e n , ~ A m p h i b i a . ~}$}

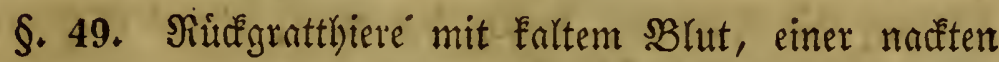
Doer von Schildern und Schuppen bedefften Scrut und wath= ren Füsen ober gar feinen Bemegungsorgnnen. Sie athmen meiftens, wenigftens in 2(rter, butech Rungen.

Der $S_{\text {Bau }}$ ber $2(m p$ bibien ift fo verichieben, Daf fich we= nig 2argemeines bariber fagen láp̈t. Das Skelet betreffent, fo fehlt allen fußlojen jugleich bis şe de n; einigen, wie ben Frofden, auch bie Rippen. Die Schildétiten haben

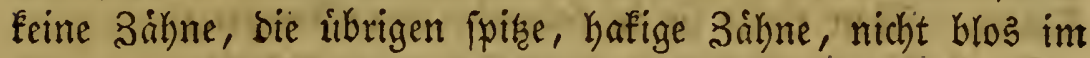
Siefer, fontorn oft auch) am Boben bes Mundes ((S) a umen=

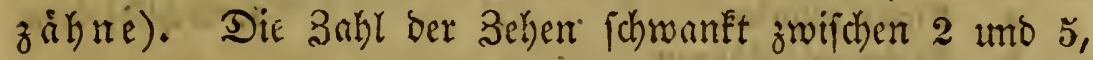
boch ift bie Yestere 3abl vortherrichend; bie im $23 a f f e r$ Yeben= ben haben Echwimmbáute zwifhen ben Behen. Den Fro=

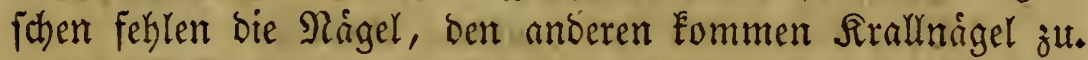
- Die ausere Bedectung ift bei Den Schilbéróten umb Jiro= kodilen aus Schildern gebilbet, bei ben uibrigen Eidechjen uno Sdhlangen finden fich Edjilder nur auf bem Sopf, fonft Schuppen, bejonders grofe, balbringformige am \$Bauth. Die

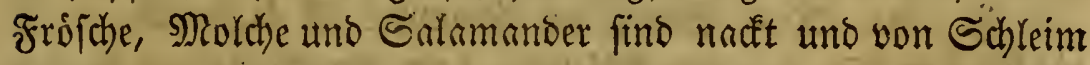
bedectet, weldjen viele Scautorijien abjundern.

Son ben inneren Srganen ift bejonbers bas 2ttymungs:

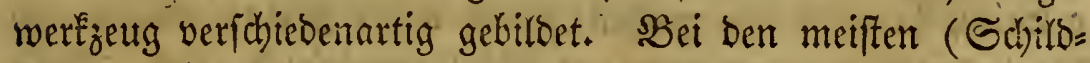
Fróten, (Fibecbfen, Echlangen) ift es eine $\mathbb{R} u n g e$, bie aber blus aus zweien groß̧en, biutigen Sacten befteht, weld)e noch baju bei ben Echlangen an (jirópe febr ungleich firto: Die nactetháatigen 2 mplibien athmen in ber Sugend, to lange

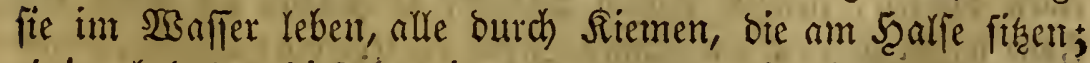
einige behalten biefelben immer, andere, wie bie Frofiche, ver= lieren fie, wenn fie bas $23 a f f e r$ verhaffen. Demnachft ift bas

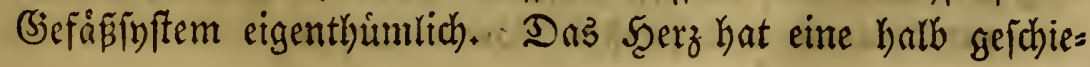




\section{Erfter Xojdhits. Foologie. I. Pitugrattbiere.}

Dene Sammer uno zwei Borbbfe *), in welche bas aus bem

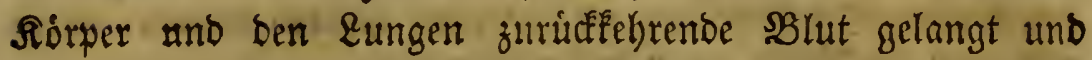
bann in bie Sammer tibergeht, von mo aus es wieber im Forper verbreitet wirb. Die Berbauungsorgane zeigen kei= ne bebeutenden 2tbweichungen, Doch fino fie fleiner, und bejon= Ders ber Darn furrjer, als bei ben \$ógeln. Dies bezieht fich auf bie Rahrung, welche bei allen, einige Schilderroten ausgenommen, thierijch ift. -

Die 2(mplibien wflanjen fich Durd) (Fier fort, bie theilz

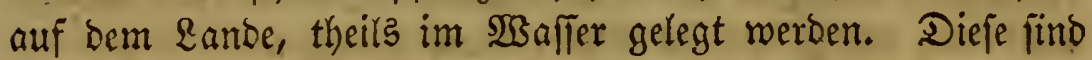
ohne Echaale. Die sungen ernábren fich felbit. Man Fennt rookl nicht mekr als 9002 (mplibien $=2$ arten, von welchen $\frac{8}{5}$

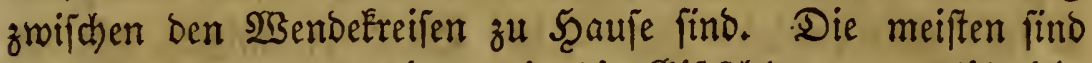
unichábliche, nur wenige, wie bie (sifitjchlangen, gefäbrliche abiere. Man theilt fie in folgende vier (3ruppen:

I. Eeib mit Sdjildern oder Sduppen bededt.

a. Siefer gahnlos. $. \because . \quad 1$. Edirberoter.

b. Silefer mit 3ábnern.

Mit 2lugenlievern, Sruftecin und vorn vers machfenen Unterkieferbogen. . 2. Eibechien. Shne 2ugentieder und 2oruftrein, Unterkies ferbogen yorn gettennt. • • 3. Edrangen.

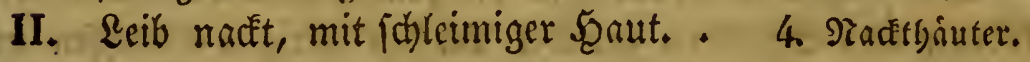

Erfte Familie. Schilbfróten, Testudinata.

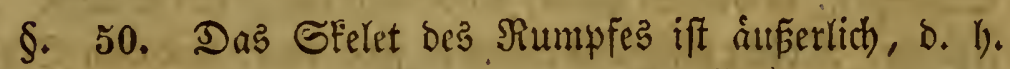

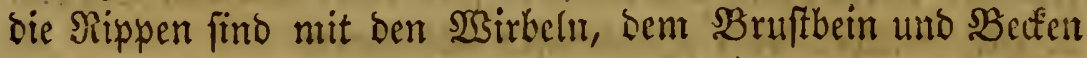
ơ einem Enochernen şanzer verwactjen, in weldhem alle (Fin= geweibe, ja felbit bie grop̃en Sinochen ber Bzeine, liegen, und

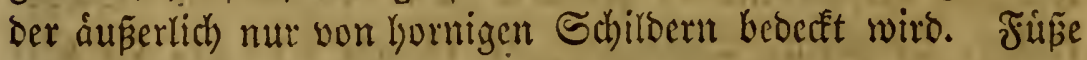
finfzehig, ftets vorkunden. Die Schildofroten freflen bejon= Ders Siriuter, bie Sceichitbfroten auth Ilfiere.

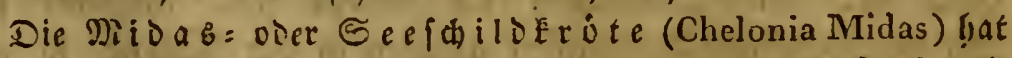
einen Ereiten, ziemlid flaigen Panjer, in welden lid Siopf und

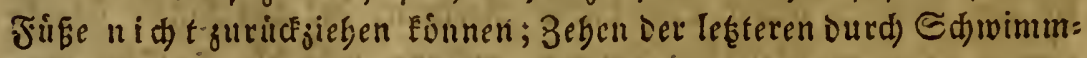

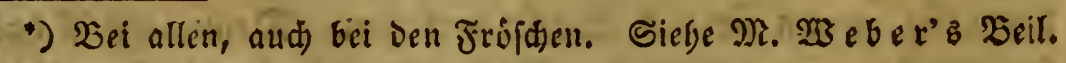
zut Znatomie und Pgyftol. כonn 1832. 8. 
baut verbunden, melft nagellos. Riscenfwild mit $z$ Reigen grofer Sornplatten unid Eleineren am Lumfange, jebe Seitenreibe mit 4 splatteri; Farbe braun und olivengrún gewellt; Fleifh grunlid. 2ltlantifder Scean; with tiber $2^{\prime}$ lang.

Die europáifhe Edyilderite (Emys europaca) bat ebenfalls einen flathen Flanzer, Der am ßande nur mentig bervors

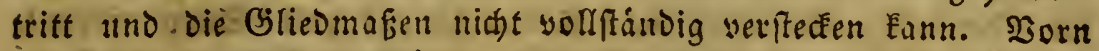

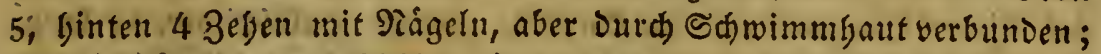
Sdmangilang. Brufffdiro bintent obne berweglide silappe. Jarbe

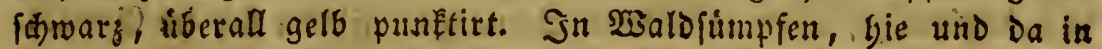
Deutichland, aud bui $\mathfrak{B}$ erlin.

Die griechifhe oder Ranofotilder íte (Testudo graeca) bat einen bogen ftark gemólbten 'Danjer, unter weldem fidh Der Fiopf uno alle B̈lieber gang verftecken Énnnen. Behen bis auf bie

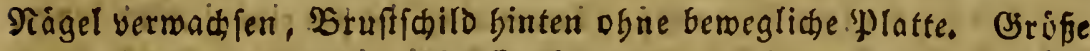
einer tudtigen Drannisfauft, Zarbe gelblid, iebes ๔aild in Der Mitte braur. Eíd = Europa und Rúftentánder des 2Rittelmeereb, in B̈ebüfden.

\section{3meite Familie. (Eibed [en, Sauria.}

§. 51. Die meiften (Eibechfen unterfheiden fich von Den Schlangen, Denen fie nake verwand fino, fith on baburd,

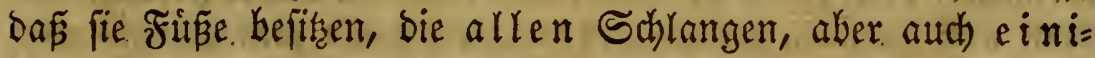
gen (Fibechjen, feblen. Utntriglidye Jennzeid)en liefert bie Bil= bung bes Jopfes, indem theils ber Sberfiefer fefter am Edja bel first, theits bie beiben Bogen bes Unterfiefers vorn verwath= jen find. Die meiffen Eibechfen haben Zutgenlieber, bie Echlangen nie. Die allermeiften Eibechjen befitien ein Bruft= bein, bie Sd)langen nie. Die (Eibechjen fino unfchádiche ahbiere, welche von Injelten und fleinen ahieren Yeben, nie-

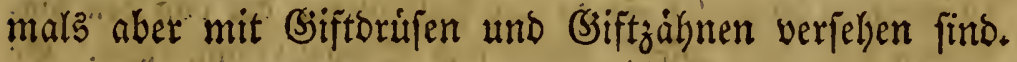

\section{A. Manzereibechjen, S. Ioricata.}

Sie haben ftatt ber Schuppen hornige, mit einem erha=

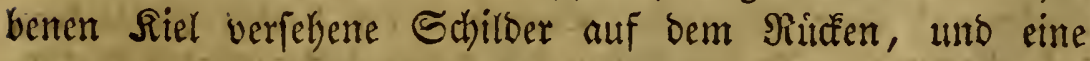
boppelte Nieife berjelben auf bent Sdhwanz.

Die Battung ber S roE odile (Crocodilus) Gat eine breite, mit Der Sichle verwadfene Zunge, eingekeilte. Zábne, eine faaltenformige Shroffnung ginter Dem 2 luge, uno vorn mit fi n $f$ freien, ginten mit vicr Durd Edfmimnibaut verbundenen Begen verfegene Jübe, Deren 
Drei innere Beben allein Ragel trager. Oer Seib if oben yon enodernen, mit eitrer erbabenen Reifte verfebenen, Sdjilbern bedect.

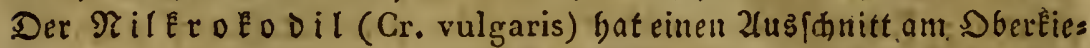
fer zur 2lufuagme Dez vierten UnterEieferzabnes, 4 : Tacken uno 6

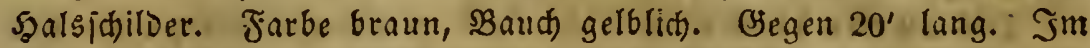
Pit. Der Si a im a n oder 2 llig a tor (Cr. lucius) Gat eine Grrube in SberEiefer, zur ?tufnabme Deffelben Zahnes, Galbe Sdyimmbaute Der Şinterfinfe, unv yier im Suadrat geftellte \$alsfhilder. 14' lang. Rord:? meriEa. Der bja vi al (Cr. gangeticus) bat eine febr lange,

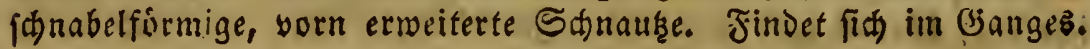

\section{B. Schuppencibechjen, S. squamata.}

Sie haben Schuppen oder eine blok marzige Şaut.

Die geme ine (Ei o c d fe (Lacerta agilis) Gat pine ziemlid) lange, tief gefpaltene 3unge, Sdilder allf Dem Sopf, einen Şalbring breiterer Sduppen an Der Feble (Şalzband), breitere, yieredige Eduppen na Baud, eine Reige von Drifen am Innenrande ber ȘinterfdenEel und ringformig geltellte, gekielte Shuppen (Wirteliduppen) auf Der Dber=

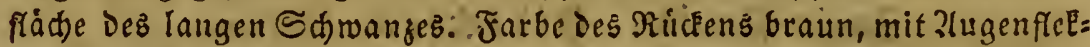
Een; am Baud gelblich ober grau, ungeflect. Bemein in bsebúfhen.

Bei dem (5 $\mathfrak{h}$ a ma le on (Chamaeleon africanus) if die 3unge ein langer, orebrunder, fleifdiger, yorn bismetlen breiter Jortfä, und Eanit nach Belieben Des Thieres aubgeftreft und guridégegogen wers Den. Diez Tlier bat grofe. Zugen, Die von ben 2lugenlieberin fo weit bedeckt find, Dafi nur Die Pupille frei bleibt; auf Dem Sheitel einen Dreifeitigen pyramidalen Fortaks. Jülße fünfzebig, zangenformig, yorn mit z wei, binten mit Drei 3eben nad augen. Shmanz lang, midelt. Reib obne Sduppen, marz̧ig. Farbe medjelt zroifden gelb, grinlid), bläulid) uno braun. Síd =2lfriéa.

Der fliegende $D$ rad e (Draco volans) bat eine Eurase, Dicke, runie 3unge; einet \{chmalen, yon beiden Seiten zufammengedruçten Reib, an weldem jederfeite ein Greiter Fallidirm, Der yon Den bin: teren Rippeir unterltukt wirb. 3eben und Saroanz lang, Dinn. Er lebt auf Báumen uno flattert. Örifífe Der gemeinen (Sidedfle. Sava.

Der $\mathfrak{e} g$ u a n (Iguana tuberculata), ebenfalls mit feitlic) $\jmath^{u}=$

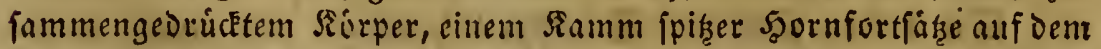
Ridén uno einer bängenden $\mathfrak{B a n m e}$ an ber Fielle; 3unge Eur b, Dick, abgerundet. 2ln Den Seiten dez Sanlies Góckerartige Gquppen.

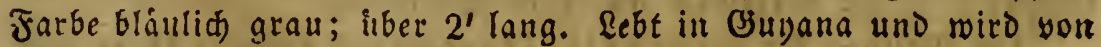
Den Cingebornen gegefien.

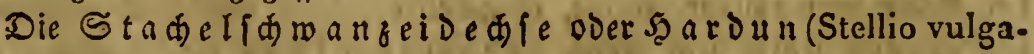
ris) Gat einen yon oben nad) unten jufammengedrúckten \&eib, Eurgen 


\section{Drttte Rtaffe. Xamphibien. Eibedjen. Shlangen.}

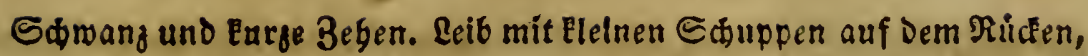
gmifden welden grófere pgramibale Sduppen fteben. Sdmanz rund, von fpligen Wirtelfauppen beElleidet. Heber 1' lang; braun.

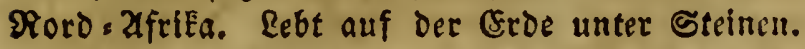

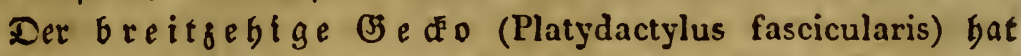
grofe Zlugen mit Eleinen Zlugenliedern, einen rundliđen $\mathfrak{s} \delta$ rper mit Eleinen ธdurppen und gang broit georidfte auf Der Soble mit Szautfalten verfefre Begen. 2luf Dem Rildén mebrere Reifen bu Drei geftelter હøuppen, Deregn mittlere pyramioal geftaltet ift.

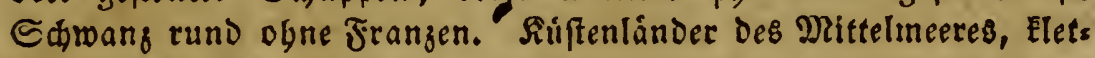

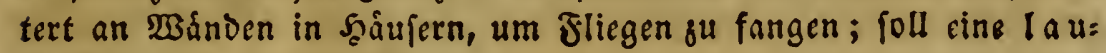
te Stimme baben (?)

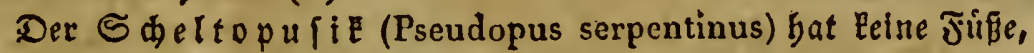
eine tiefe Jurde an beiden Eelten deg Baudes, an Derem Ende ein Forffaks, Der Die Şinterbeine andeutet. Bunge Elein, flä, nađ yorn verengt. $3^{\prime}$ Lang, braun, Sdjuppen breit, glatt, wirtelformig,

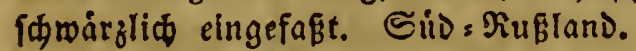

Der SEinE (Scincus officinalis), mit flacher, etwas Gervors ftebender Edfnauşe uno vier Jüífen mit breiten, gefranzten 3efen zum Braben. Zunge Elein, am Enve allzgefdnitten. Sduppen

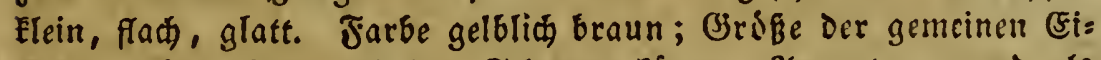
Dedfe, aber Dider uno Der Sdyang kurber. Hegypten; ward als Seeilmittel gebralldt.

Die $\mathfrak{Z}$ Iind fiflet $\mathfrak{e}$ (Anguis fragilis), mit gleider 3unge und Єduppenbiloung, ogne Baudringe (melde Den હđlangen eis gen find) und ogne Jüpe, nit Iangem, f aber Deutliden 2(ugenlievern. Siupferfarbell mit DunElerem Rindén: ftreif. Bemein bei uns in Bebúfhen auf Wisfern.

C. Singeleibechjen, S. annulata.

Sie baben fchmale, ringelformige (siurtel ftatt ber Sd)up: pen, bie burch feine Sinnen in lauter vierectige Felder ge= theilt find. -

Die Sandeidedfe (Chirotes canaliculatus) bat $y$ ier $\mathfrak{f} \mathfrak{e} \mathfrak{b}$ i:

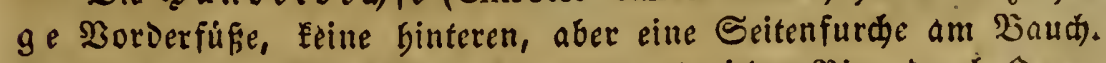

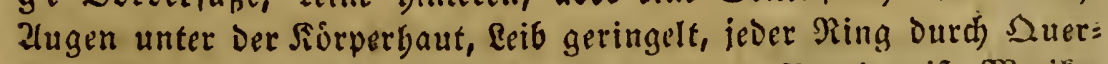
linien in viereckige Jelder getbeilt. Farbe braun, Sauá) weif. Merié.

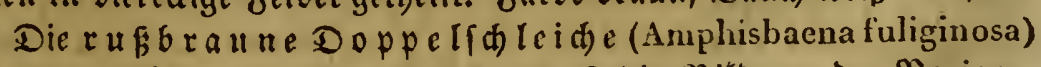
bat Eeine Jufe und Eeine Furde, fonft Die Billoung Der $\mathfrak{Z}$ origen.

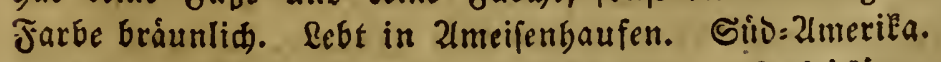

\section{Dritte Familie. Sallangen, Ophidia.}

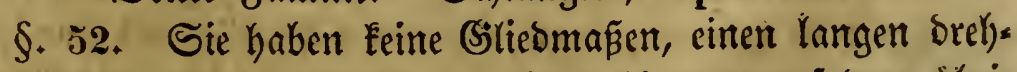
rumben $\mathfrak{L} e i b$, ber auf bem Sopf mit Sdjildern, allf bem übri=

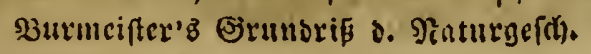


gen $\mathfrak{L}_{\text {eibe }}$ mit Schuppen, am $\mathfrak{B}$ auth meiftens mit Scalbringen bebecft ift. Die beiden Scalftin bes Unterfiefers fino vorn nicht verwadefert, fondern mur burch ein fefniges sBand ver= bunden; Daber entftebt bei gefdhloffenem șaule an ber Seble eine Furche, Sinnfurche, bie in ber Regel an jeder Seite mit zwei grosen Gchildern ( Rinnenichilbern) begringt ift. Durch bie 2umejenteit biejer Furche ober beren Schilloer, uno ourch Den Nangel ber 2ugenlieber, untericheiben fich bie Schlan= gen ficher von ben fuflofen (sibechfen. Die meiften Schlan= gen fino unfa)idolich, einige baben (siftzálne uno (Siftoruifen. -

A. Mit grofer, weiter Munooffmung (Eurystoma).

a. Slune (siftzáhne, mit fdomalem Sopf.

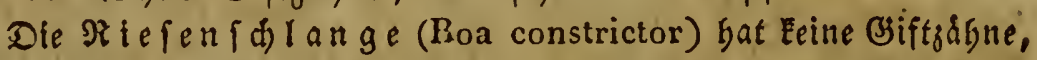
einen nidt mit Sdildern, fondern mit Sduppen bededten Siopf, Eeine Rinnenjdilder, aber etnen Sporn neben Dem 2lfter. Farbe róthlid grau, mit einem Streif úber Den Sopf und einer gezackten Ruckenbinde, auf meldier gelbe Fleden. 23 is $30^{\prime}$ lang. Brafilien.

Die $\mathfrak{R}$ ingel a t ter (Coluber natrix) bat Sdilder auf Dem Sopf und neben Der Sinnfurde, und parige હdilder unter Dem Sđwanz. 2luf Dem siukén geficlte Sduppen. Farbe Dunkel fdmar: grau, baudringe mit meifiem Seitenfecé, auf Dem Rilcken fdwarge PunEte, jederfetts am Sainterbaupt ein beller Mondflec. OSemein

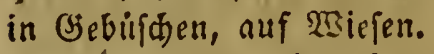

b. Mit (sififállunen uno einem nach binten breiten, oreifeitigen Ropf.

Die al a p per $\{$ lange (Crotalus horridus), mit breitem Sopf, ouf Defien Mitte eine von હdhilDern eingefafte Bertiefung; unter Dem Sdmanz unpaarige Salbringe. Sm Maule am Sberkiefer mebrere gebrgene, ftarke, Durdbobrte 3äbne (Bjiftzábne), nus weldhen beim Bif́ Das Giift bervorquillt; an Der Sdmanz̧pişe Die Silapper. Jarbe braün, mit Dunketeren beller gefaumten Diúčenfleçen. Siid: Imerifa.

Die gemeine $\mathfrak{B}$ iper (Vipera berus) Gat Shuppen allf Dem Sopf und yorn einige Sdilder, eine lánglide, fenEredte Pupille und

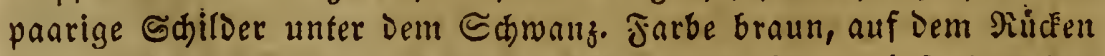
mit Doppelter, fdwarzer 3ickadebinde. In Gebuifhen, befonders Dez mittleren Deutfdlanos. Gebiert lebendige Jungen.: 2' lang.

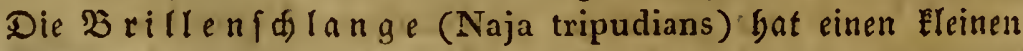
mit Sdildern bedecten Siopf, eine runde 'Pupille, und eine breite, flade, runde Ermeiterung Des 5 alfes, auf welder Die 3eidnung einer Brille ftebt. Farbe gelbbraun, 3-4' lang. Dftindien. 
Die Ma afferfiflange (Pelamys bicolor) bat હdilder alf Dem Sopf, abcr Éeine Şalbringe am Baud); ๔dhmanz feitlid bufaul: mengedrúcti, abgerumbet, wie eine Jloffe. Farbe oben braun, ant

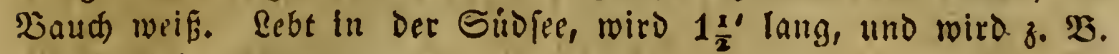
auf Drabelte gegeffen.

B. Mit Fleiner Mumboffinung (Stenostoma) und flci= nem Sopf, ber nicht breiter ift, als ber Şals. Snmmer vhne

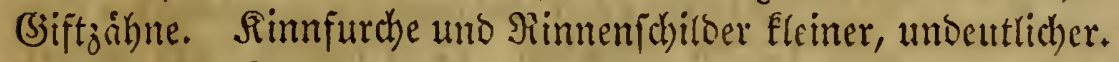

Daz 3 ló a uge (Typhlops lumbricalis) hat eine fumpfe

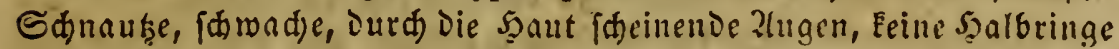

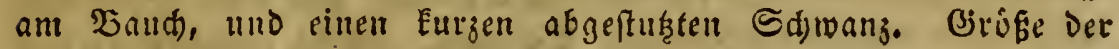

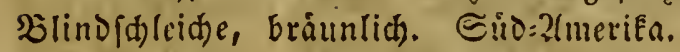

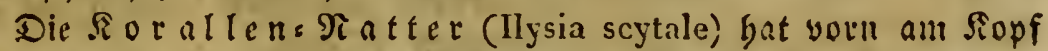
breite Sdilder und grófere Edjuppen an Baud; Jarbe Eorallroth

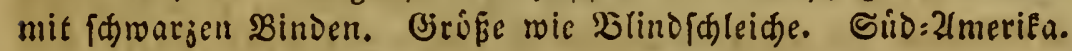

Bierte Familie. Frofbamphibien, Batrachia.

§. 53. Sie baben sine nactete von Schleim bedectete Scaut, beren Saleim von vielen Scrutorifen abgriontert wirb. Shle (Fier find olne Sd)ale und merden ins 23 nffer gelegt, wo auth bie Sungen bie erfte Beit ihres $\mathfrak{l}_{e b e n}$ wohnen. So lange

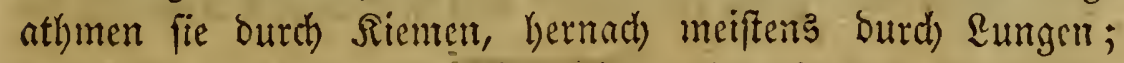

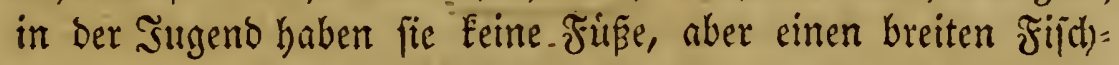
fchwang.

A. Ungefdyanjte Frofdamplibien (B. anura). Sie haben im 2lter keinen Sdhwanz, und vorn 4 binten 3ehen. Die Suntgen fint bie furgen, bicken, mit einem brei= ten Schwanz veriehenen $\mathfrak{R}$ aulquappen; fie befommen bic Scinterfitipe juerft. Faft alle baben eine laute Stimme.

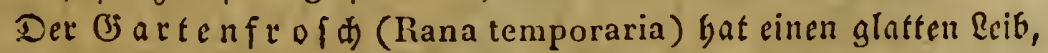
Eeine Dbrorúfen, fpişe 3eben mit Sdbwimmbauten zmilden Den Gin: teren. Farbe braut, ant Bauds gelblid. Bemein.

Der \& a u $\mathfrak{f}$ fr of aber \{deibenfórmig erweiterte 3ebenfpizen. Jarbe grabgrún, Daud) meis.

Die I $r$ i te (Bufo cinereus) Gat eine margige Szaut, grofe Dhr:

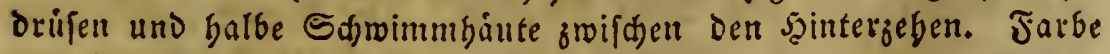
graubraun. Szat Eeine laute Stimme.

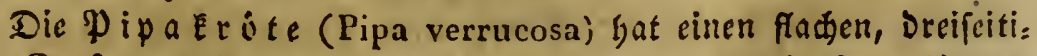
gen Siopf, uno Bordergelgen, Die in 4 Spigen aublaufen; bintere 


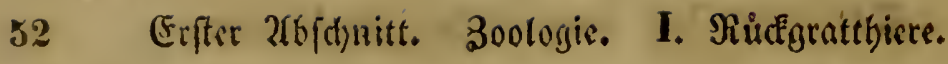

nit gauzer Sdmimmbaut. Daz M̧etbden trágt Die Jungen auf Detm Rúferer. Surinam.

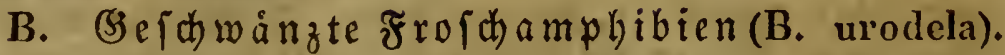
Sie haben aud im 2flter cimen langen Schrwan. Dic Jum= gen betommen zuerif bie Sorderfúßse. Manche leben beftín= big im $\mathfrak{3 a f f e r}$ uno belalten bie Siemen zeitteben3.

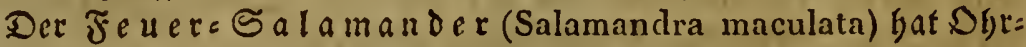

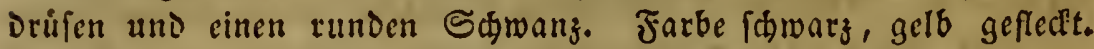

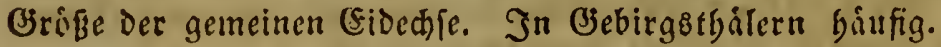

Der $\mathfrak{S}$ a $m \mathfrak{m}=\mathfrak{N}$ old (Triton cristatus) hat keine Ohroviffen uno einen zufammengeorickten Edwanj. Farbe graufdrear, ain

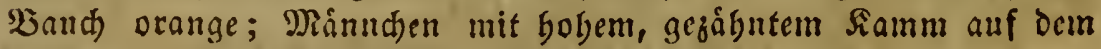
Sildere. In Teiden uno auf feudten $\mathfrak{B B i e f e n . ~}$

Der $\supseteq \mathfrak{l} \mathfrak{m}$ ('roteus anguinus) bat Eeine 2 ugen, beftåndig blei: bende Siemen am Salfe, mo vorn 3, binten 2 3eben. Farbe bell= rittlich. Ecbe in unterirbifden Geewdffern, befonders in Der 2bels: berger Brotte in Sirain.

C. Die fdrangenartigen Frofdamphibien(B. anguinea) baben Éine F́físe.

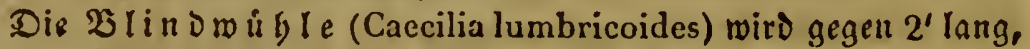
fingersbide, bat ein unter Der Szaut verftedtez $\mathcal{Z}$ (uge, und mur in Der Jugend siemen. Farbe rufbraun. Rebt in Sumpfen 2urcrizas. -

\author{
Dierte Rialfe. \\ Fif be, $P$ is c e s.
}

§. 54. Sie athmen burd Sitemen, haben einen nacten,

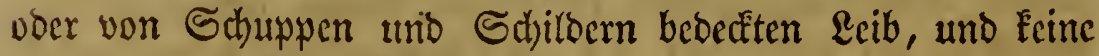
oder floffenfúrmige (slicomafen.

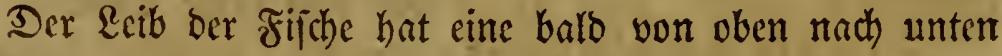

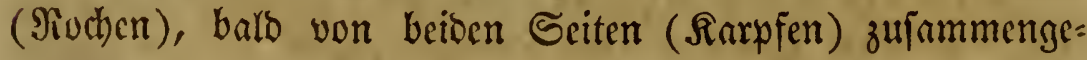

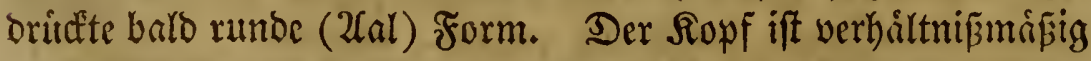
groß, uno unmittelbar mit bem ßiumpf verwachfen, fo baß ber Şalక fehlt. Der Soberficfer ift beweglich uno meiftens, wie Der Unterfiefer uno (Saumen, mit fpizen Bâhnen bewact) fen.

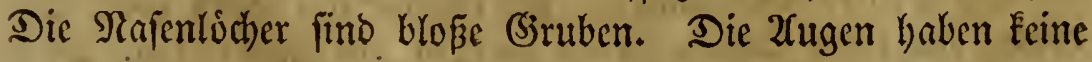

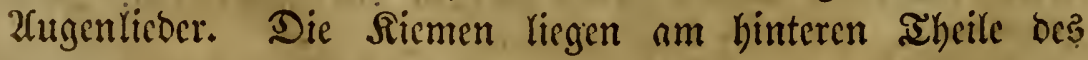
Sopfes, beftehen bei ben Einen auts Énmmfórnig aneinanber gereifeten Blittchen, bei ben 2fndern aus Buifcheln von Fis: 
oen, bie an bem Siemenbogen feftitien; nod Xnoere baben zugleich an ber åuperen Scaut feftgemad) fene Riemen. BBi jenen weroen bie Riemen von einem großen, firoot)ernen, aus 4 Stücten beftekenden $\Omega i$ e mende effel verbeft; bei biefen bit=

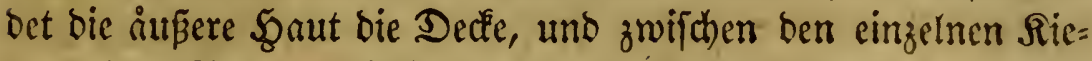
men find Spalten, bie in bie Siemenhoble führen. Die Fifche

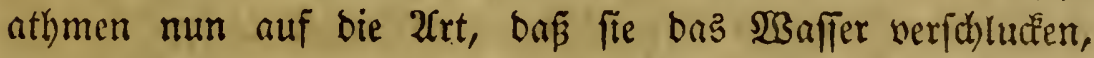
und bei geidlofiènem Drunde aus ben zwijhen ben Riemen befindlichen meiten Deffnungen, wobei ugleidh Der Siemen= Decker geoffnet miro, hervortreiber. Das Scerz licgt binten unb unten jwifchen ben Siemen, faft an ber Selde, uno beftelyt nus ciner Sammer und cimem Sorhof, in welchem alles \$llut, bas ats bem Siorper zurúdflommt, fich ergießst, bann in bic Sanmmer fließ̄t, von biejer in bie Siemen getricben wirb, aus welchen es in ein großę $\mathfrak{u}$ ber ben Sitemen gelegenes (siefíp,

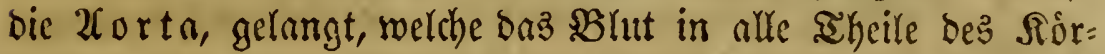
perz leitet. Die Fif he baben alfo mutr cinen Rreizlauf, bie warmblütigen Rindgratthiere zwei, indem bei ihnen bas \$Blut

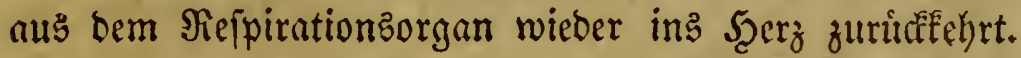

Die Floffen ber Fifche beftehen aus vielen theils ein= fachen ftacheligen (Stad)elfloffen), theils am Ende ge= fpaltenen uno gegliederten (203eid)floffen) Strablen, zwi= fichen welchen eime weiche Scaut auşgeipannt ift; bie unteren Enoen ber Strablen find an im Rörper verftecleten Inochen

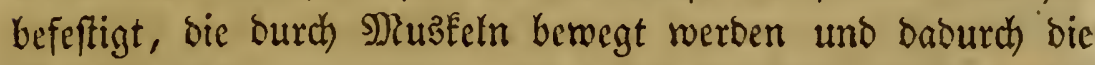
Floffe in Bewegung feken. Einige floflen fiţen an ber siult=

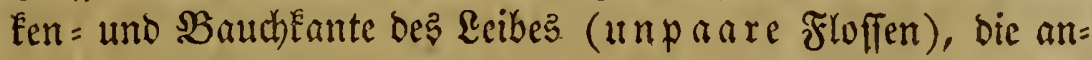
Deren bopyelten Flofien (paarige Fl.) fiken neben bem Jopf uno am Bauch. Sie entfprechen ben (şlieomafen ber frúber betrachteten ahbiere.

Im Snneren vieler Fifche bemertet man eine längliche,

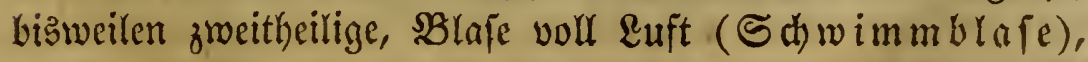

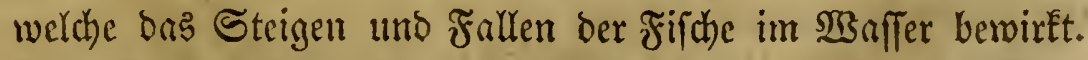
Der Darm ber Fifthe iff furz, bie $\mathfrak{E} e$ ber großß. Die Eier= fiufe bor 2 Seibchen (Foogen) enthalten fehr viele (Firr (oft ge= 
54 Erfter 206id)nitt. 3oologie. 1. Siuctgrattbiere.

gen 30,000 ), und munden neben bem 2 ffter, ber am (5noe ber Bauthbible befindlich ift.

Die Fiiche leben nur im $23 a f f e r$ und fterben, wenn fie lángere Zeit außerbalb bes Masaffers gebalten werken. Sie

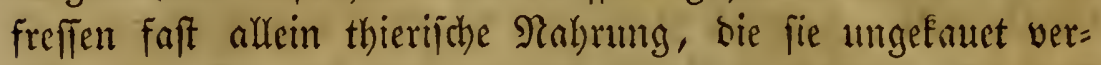
fchluden. Man theilt jie in folgende Familien:

I. Fiiche mit querer Mundoffnung (Plagiostomi).
A. Mrit fnorpeligem Sfelet.

B. Mit Enidhernem Sfelet (Ostacanthi).

a. Sberḱiefer am Edjidel feftgemadfen. 2. - Şafttiefer.

b. Dberkiefer berweglich.

- Siemen bifdelformig. . : 3. - Suaftenkiemer.

" Siemen Eammiformig.

Mit geglieierten zerfdilifienen

Etrablen aller J̃offen. . 4. - 2Beidfoffer. Mrit ftadeliden Strablen Der

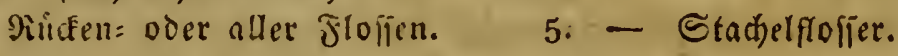

1I. Fifche mit rundem Maul zum Saugen. 6. - Nunomáuler.

Erife Familie. Sinorpelfifd)e, Chondracanthi.

\$. 55. Sỉ baben ein weiches, Enorpeliges Sfelet, uno eine nadite, ober von fleineren ober gróseren cinzeln ftehenoen

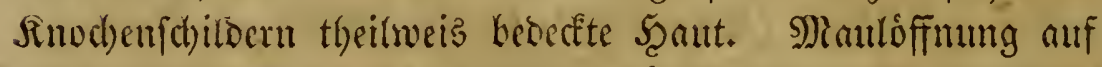
ber unteren Seite des Supfes, hinter ber hervorragenden Sdhunutze. SBruft = umb SBand)floffen fehlen nie. Şieker:

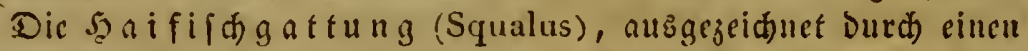
rukilidyen scib; eiuen Diffen, runien Sdman ; fủnf Siemenlo:

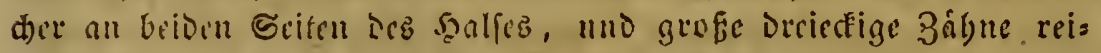

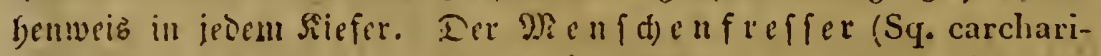

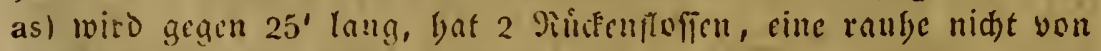
Edjitdern bederfte samt und cime graulide Farbe. Lebt in allen Meeten. Der $S_{\text {a }} g$ e fif d) (Sy. pristis) bat tinen langen, fowerit:

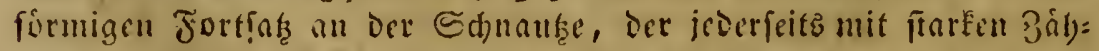
nen befest ift. 15' lang. Shittetmeer. Der Sgammerfifd) (Sq. Zygaena) bat einen nach beiden Eeiten in einen Jortfaks, an mel: dem die 2 lugen figen, ausgevegnten fiopf; ex roird gegen $12^{\prime}$ lang uno lebt im Mittclmecte.

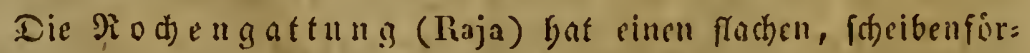
migen Reib, mit weldem bie grofen, fligelformigen bruffifofien

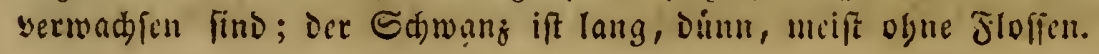


Jänf Siementocher an Der unteren Eeite binter uno neben bent Maule, auf ber oberen die Ulugen. Die gemeine Diod) (R. batis) viereckig, rhombifd, mit langem, Dünnem ङdgwang olne Jlof: fell, 2' treit, $1_{2}^{x \prime}$ lang. Norbfee, miro gegeffen. Sie 3 it terto: de ( $R$, torpedo) bat einen runien, Ereisformigen Reib, einen die: Eeren, Eirjeren Eabmanz mit Fiofien. Farbe rotbbraun, oben

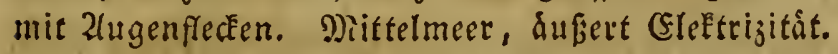

Die $S_{t} \mathfrak{i} \operatorname{gattung}$ (Acipenser) bat einen runden Reib, etne

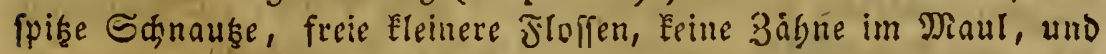
eine n grófen Siementedfer mit freien Siemen. - Der S tór (A. sturio) Gat fünf ßieigen gropere, flader Sinodeniduppen, uno Elei=

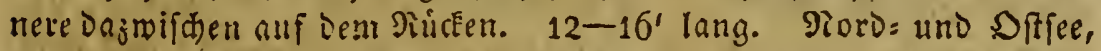
gelft yon ba in die groferi Jliffe. Der $\mathfrak{5}$ a u fen (A. huso) bat auf Dem Siúcten viele Éeine fternformige Sinodenfajilder zrvifhen Den grofen. In Der Mzolga, Donn, fdwargem Niere. Zluz Der Edbimmblafe verfertigt man die Şa ufenblafe, Der Rogen ift fiaviar.

\section{3weite Familie. Scafteiefer, Pectognathi.}

§. ว6. Shr Sfelet iff Enóchern, uno Der Sberfiefer unbeweglich mit ben Sippffnodhen verwachjen; ber von Schmelz

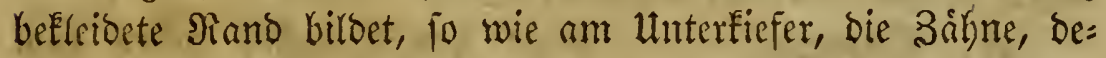
ren 3akl nicht bebeuteno. \&eib Eurz, nackt, ober mit Stracheln oder Edjildern bebeckt. $\mathfrak{B} a$ uch floffen fehlen. Siemendeckel

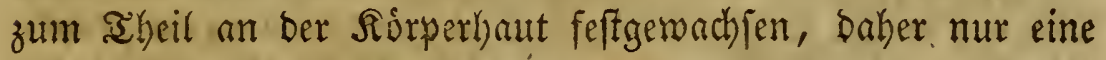

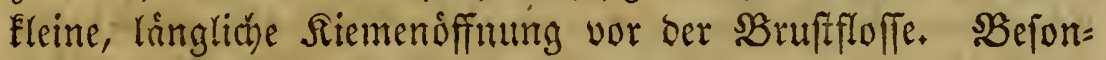
bers in futslid)en Gewaillern.

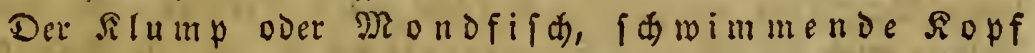
(Orthragoriscus mola), Sileferrano bildet in jeben Siiefer einen cinzigen 3 algn, Der von Den feiffigen Sippen nidft bedeckt mird. Reib iduppenlos, bod, fdeibenformig, ogne Єdyant, mit bober

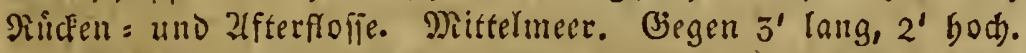

Der Siofferfifd (Ostracion cornutus) bat mefrere Eegel= formige, jabnartige Jortfäk̨e am Siande icies Riefers, und einen

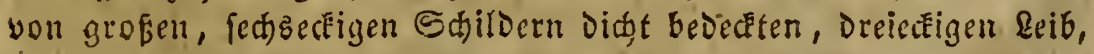
Der an Der Etim und binter Dem Zlfter mit 2 Stadern bemegrt iff; Faum $\frac{3}{4}$ lang, róthlidbraun. Sndifher Dcean.

Dritte Familie. Duaftenkiemer, Lophobranchiati.

§. 57. Sfelet Enochern, seib von Schirbern bicht beberft,

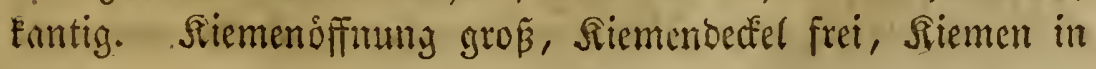


Büfcheln am Riemenbogen; Ropf fănabelförmig verlångert. Leben nur im Neere.

Die Reernadel (Syngnathus acus) bat einen langen, Duns neu, nach binten verjüngten, fünfeçigen Leib, febr Eleine Bruffflofien, aber Eeine Baudfloffen, und eine rundlide Sdymanjflofie; braun= lid); $\frac{2}{2}-\frac{3}{4}$ lang. Oftéc.

Das S eepferoden (Hippocampus brevirostris) bat cinen

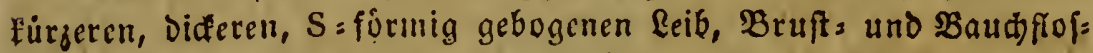
fen, aber Eeine Schmanbflofien. 3" lang. Dittelmeer.

Der $\mathfrak{D}$ ra de $\mathrm{n} f i f d$ (Pegasus draco) bat einen wirfelformi: gen Reib, grofie fád)erfórmige Bruftroffen, keine Baudflofien, uno einen Eurgen, Eegdformigen Sdwang mit einer Radens und EnD= floffe. Sindifder Dean.

\section{Bierte Familie. $2 \mathfrak{B}$ eidffloffer, Malacopterygii.}

§. 5s. Sie baben bie vollfommenfte Firchgeftalt, ein

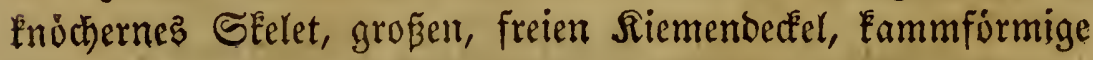
Siemen, und weiche, geglicoerte, am (Ende zerichliflene Strab)= len. Slach ber Stellung uno bem Mangel ber Bauchfloflen laffen fie fich wieber eintbeilen:

A. Bauchfloffen an Ende bes $B$ auchës neben bem 2ffter. $B$ a u (b) floffer, M. abdominales. Scierber geboren:

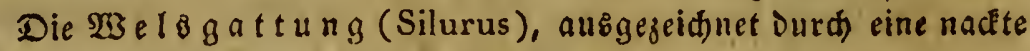

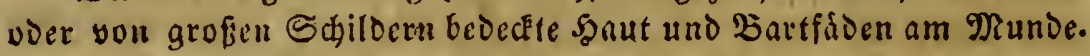
Reben meift in Flúffen. Der geme tne $\mathfrak{B e l}$ (S. glanis) bat

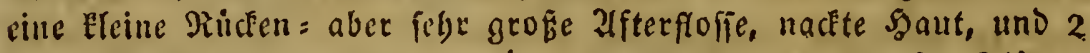
lange כartfäon am Dbers, 4 Eurge am Unterticfer. In Jliffen

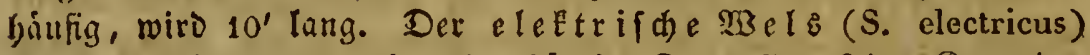
bat Eeiute riuctinfoffe, aber cine bautige Jettfofie auf Der Siante des

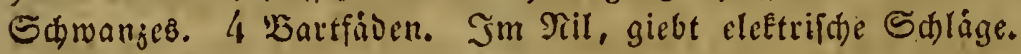

Die farpfengattung (Cyprinus) bat eitue riuckenfloffe über Dem 2ffer, zirmlid grofie ङduppen, meiftens Bartfáden und feine 3ágne. Der ge me i ne Siat p fen (C. carpio) bat 4 Bart= fáocn unb eine grufie Siucfenfloffe, Deren zweiter und Dritter Strabl ein einfacher, gezdonter Stadjel ift. Jarbe braun, Baud gelblid. In Jliffen. In unfern Jliffen leben gegen 20 ?tren Diefer Battung.

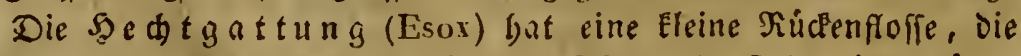
binten auf Der Edowangkante liber Der afterfloffe ftebt, einen läng:

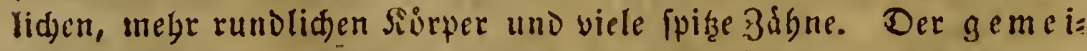
"e Şedt (E. lucius) bat einen breitgeoridten, abgerundeten sopf und ciuge grofie $3 \not$ gne in Unterfiefer. Farbe grüngrau, 
gelb gefledt, Baud meißlid; bismeilen gegen $4^{\prime}$ lang, in ber Res

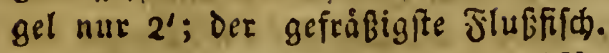

Die Sering gattung (Clupea) bat ebenfalle nur eine Eleine Ruictenfloffe, Die úber Den Baudffoffen ftegt; grobe Sduppen, bie fegr leiøt abgeben, eine aus fágefórmigen Sdhuppen gebildete Baudeante, uni einen breiten, ftark nad unten gefogenen Dber: Elefer, úber welden Der unterliefer binausragt. Der ge meine Syertng (Cl. harongus) bat einen. (fimmalen, ftark zufammenge: orúçten eeib, Der am Baud mit bervorragenden, großsen Єdups pent befeģt iff. YEBird 1' lang, ift fllberfarben mit blaugrúnem

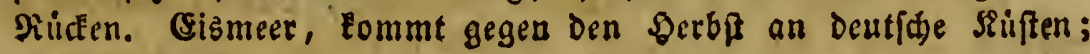
ift dufierif zafleeid.

Die $\mathfrak{L}$ a ds gattung (Salmo) bat die Form und Den Sau Der Şeringe, unterfacidet fith aber Durd eine Eleine, ftrablenlofe

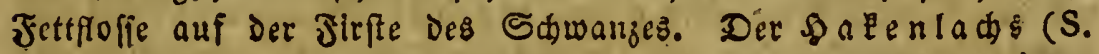

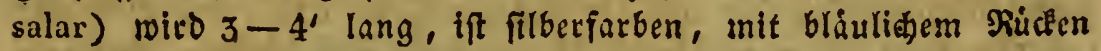
uno einzelnen fownarzen Flecken. Eer lebt in Meere, gebt abex jur

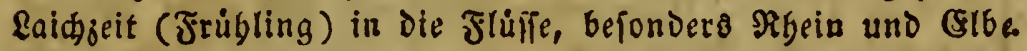

B. Baudbfloffen vor ben Bruftflofien, bei manchen an Der Reble. Reblfluffer, M. jugulares. Dahin:

Dic Dovfd gat tung (Gadus) bat Eleine, lảnglide $\mathfrak{B}$ rufts floffen uno nod Eleinere $\mathfrak{B a u d f f o f f e n ;} 2$ bis 3 gleidje, Eleinere, oder eine grofe Rúclenfloffe und einen langgeftrecten, mebr tund:

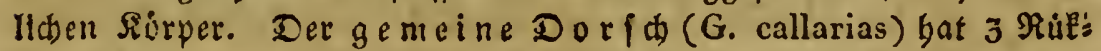
Eenfloffen, wovon die mittelfie die grsfite, und zmei 2tfterfoffen. Qeib bráunliđ, mit Dunêteren Dunten. Sopf zugefpişt mit Bart:

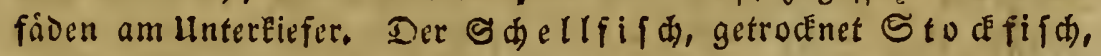
gcfalden und getrocinet silippfifd (G. aeglefinus), bat aud Drei giudécufluffern, aber die erfte Eleinfte ffegt Der zmeiten fegr nabe. Sepp ftumpfer. Sartfäden Eurz. Eeib cinfarbig gelbbraun. Nord: fee. Die Il a lquappe (G. lota) bat eine Eleine und eine fegr

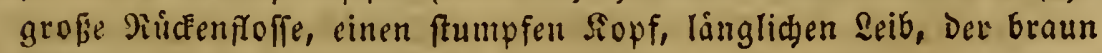

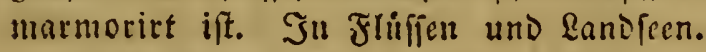

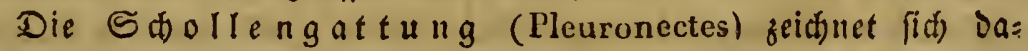
Durd) yor allen Riulgrattbieren aแz, Daß ilgr Reib unfymmetrifa iff. Ees fteben nebmlidy beide Zugen auf Der einen Eeite und Das Mant ift fajief von oben nadh unten gezogen. Die Sdjollen baben Die Dauchfoffen unmittelbar an Der Sirfle, Dicht neben sinander,

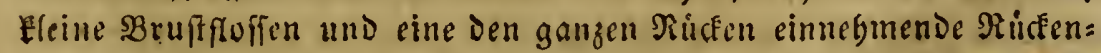
flofie. Iffterfoffe an Der ganzen Uluterfite Des Edjmanzez. Sie leben nur im gieete, liegent meiftens auf Dem brunde und fandm:

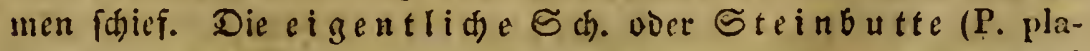
tessa) bat Die $\mathfrak{T}$ ugen an Der redten Evite, einun faft runden \&eib 
mit Garten Stadelfduppen und ritgliden Flecten. 1'lang. Dber: feite Graun, Baudfeite meib; fo bei allen. Die Flun ber (P. flesus), länglidher, kirjer, faft glatt, mit Stadbelfduppen neben ber Ecitenlinie. Tord: und Dfffee.

Der $S_{\text {diff }} \mathfrak{b}$ alter (Echeneis remora) bat einen langli: chen, fommetrifden \&eib, febr Eleine Eduppen in Der Dicken Saut, und auf Dem Sopf eine grobe, in Jelocr getbeilte Saugideibe, mit werder ex fich fifflangen fann. $1 \frac{*}{2}$ lang, bráunlid. "Sm atlanti= inen Scean.

C. Bauchflofien feblen, Reib lang, dimn, runolich.. Sablbiadbe, M. apodes. Dahin:

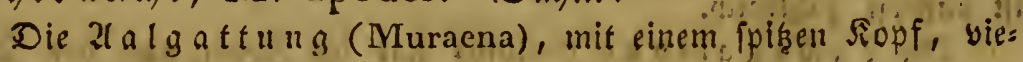
len Eleinen 3ínnen in jedem Siefer, uno einer-Eleinch Siemenipalte yor Den Sruiffoffer. Der geme ine 2lal (M. anguilla) bat eine

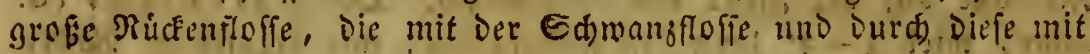
ber afterfloffe zufammengangt, Baud filberweif, sincén, idsmarz: grau. Dir 3 ittera al (Gymnotus electricus) bat einen rundi= den Fopf und Eeine viuckenforfe. In T̈luffen und Eeen Sủd:

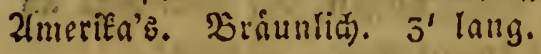

: Funfte Fanilie. Stachelfloffer, Acanthopterygii.

\$. 59. Sie briben ganz die Geftalt und Bildung ber Borigen, aker bie Strablen ber গinden = und 2ffterflofle, bis: weilen nur bie vorberen, beftehen aus einfachen, ungeglieberten Stad)eln. Sie Bauchfloffen ftehen meiftens in ber lakge ber Brufflofien, theils dicht dabinter, faft barunter ( $B$ ruffflof fer, A. thoracici), theils bavor, mebr nach ber Aeble zu (Jeblfloffer, A. jugulares). Diefe (Siruppe ift bie zahl= reid)fte won allen Fijōfamilien, fie entbalt:

Die Bruppe Dex Tf u Durd einen mit fibr Eeinen $\Xi$ duppen bededten, oft fabibenformi= apen fraden, oder enlindrifden Reib. (Fine grofie oDer 2 Eleinere iiickenfoffen, meiftens yorn mit felje langen Errablé, Die binteren Etrablen oft frei, obne Şaut. Der eigent lid e $\mathfrak{T}$ b. Scomber thynnus) bat zmei genaberte Silckenfloffen, Die bintere in viele freie Strablen aufgeloft, und an Den Seiten Des Sdmanzes ein Sorn= blittchen. Es wird gegen $12^{\prime}$ lang, ift ftablblau geforbt und lebt iun Mittelmeer. Die Di a $\mathfrak{E}_{\mathrm{e}}$ le (Sc. scomber) mird Eaum 2' lang, bat zmei fern ftebende Siuckenfrofien und fonft die fiennzeiden bes 3origen. Forbfee. - Der $S$ d wer $D$ t fif d (Xiphias gladius) un: terídeibet fich auffallend Durch ben idmerdtformig verlangerten 


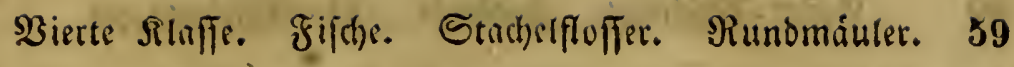

Dberfiefer und Den MRangel Der $\mathfrak{B a n d f f l o f f e . ~ E r ~ w i r d ~ g e g e n ~} 5^{\prime}$ laug, bat nux eine, grofe, yorn bobe giúcfenflofie, und einen runven Reib. Jarbe meiǵgrau, Ricken DunEler. DAffee, giordfee, Sittelmeer.

Die (Sattung Der Sitipy fifde (Chaetodon), mit runolidem,

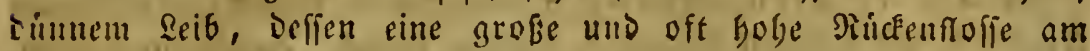

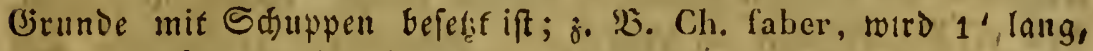
ift filberglánjeno bis bleigrau, mit blauen Sucrbinden. Zttlanti= Ther Deean. 2(ud) Die Übrigen Ztrten Dicfer Sruppe Teben nur in Der, See, befondere zrmiftén Den Wendeereifen:

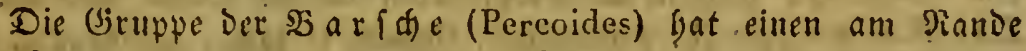

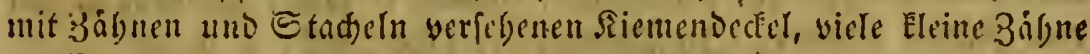
im Dauno uno grofie Eduppen. Neiftens' 2 Rúcfenfoffen, yon Denen nur bie voriere ftachelid ift. - Der ge meine 2 . ober

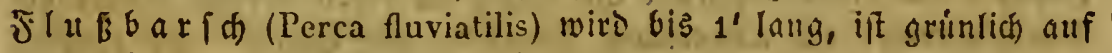

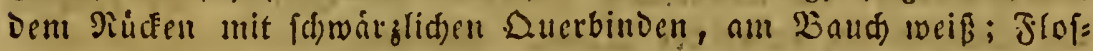

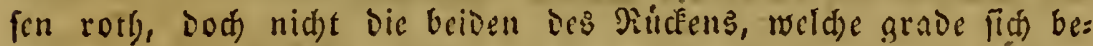
rúbren. Der SanDer (P. Lucioperca) bat cinzelue, grópere Bälyne,

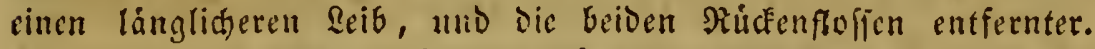
Farbe grau, auf Dem siúcten fdunár

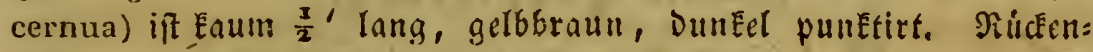
floffen verwadffrr. Zllle 3 in unferen Giewásfern.

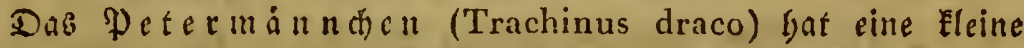
sorbere Siludenfloffe, einen ftarkent Stadtel ain Sitemenoedel, uno

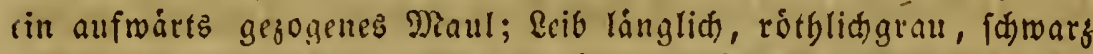
geffectit, Eaum 1' lang. 2(n curopáifden Siüfetr. - Der $\subseteq$ tern= fe her (Uranoscopus scaber) bat einen Diffen Siopf, Defien 2lugen

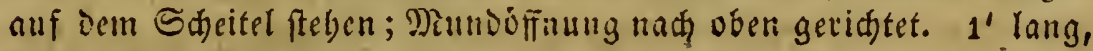
bräunliád. Drittelmect.

Der Etichling (Gasterosteus pungitius) bat freie Etadjeln yor Der Rickenfofie und cben foldhe ftatt Der Baudffoffe. Reib klein, Nackt, mit fritliden grofien Swilbern. In Bäden, gemein, $1 \frac{x}{2}$ " lang.

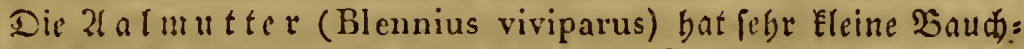

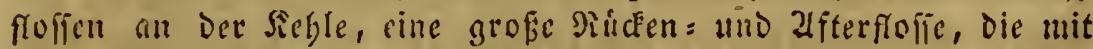
Der fpişut Ed)wanjfofíc jufammentángt. Eduppen Elein, in oer

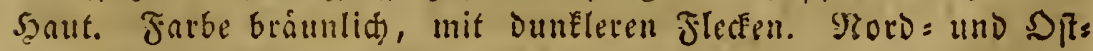
fic. Usebirrt lebendige Jungen.

Sechbte Familie. Siunomåaler, Cyclostomi.

§. 60. Sic haben ein rumbes, zum Saugen eingerid)te= tes Naul, bas an bem breiten, abgeftusten, meift tridterfor = mig vertieften Sorberende fict) befindet, uno bariber einen in 


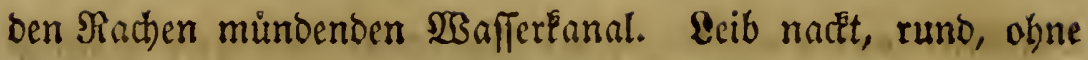
Schupen, leine panrige floffen, Eeine Siemenipalte, fondern Siemenlód)er an ber Seite bes Jiorpers. Sfelet fnorpelig, fehr weich, Eeine Rippen. Dabin:

Die $\mathbb{\Omega}$ mpretengattung (Petromyzon), Drund trichterformig erweitert, mit baligen 3\&bneu in Sireifen befeşt. Yugen vorbanden, viele (z) Fiemenloder an jeder Eeite. Midenfioffe vorbanden. Die eigentllate \&. (P. marinus), wird gegen $3^{\prime}$ lang und $1_{x}^{2} "$ Dick, Frarbe gelblid, Ruden graugrün mit braunen Flecken; 2 Rid"enflof= fen. Rorder, gebt in die Glbe, felbft in die Saale und Babel. Das $\Re$ e u n a u ge (P. Auviatilis), Eleiner, 1' lang, gelbbraun, auf bem Ridien Dunller. In Flüflen, Der Dier, elbe u. a. m.

Der $S \phi l e i m a a l$ (Gastrobranchus coecus) bat Eeine 2ligen, Franjen um die Sauggrube am Siopf, an Deren oberem rande Die Sprikiffnung; ein fiemenlow jederfeits am Baud. Er fondert viel Shleim ab, Dafer der Orame. SRurdice.

\section{3ิweite Sauptgruppe. \\ (S) $l i$ e d e r $t \quad b$ i e $r$ e.}

§. 61. Das charafteriftifhe IRettmahl ber (Silieocrtbiere liegt in ber allgemeinen (Sieftalt bes lforpers. Diefer ift nehm= lich in mebrere binter einander liegenbe, gleiche ober ungleiche Singe getheilt, uno trågt in ben allermeiften. Frillen an eini= gen ober allen biefer Ringe bentlicbe, wicberum geglieberte Fǚe, oft aber noch anderweitige Bewegungsorgane.

Die (Sieftalt bes Siorpers wiro baburch eime febr be= ftimmte, onß̧ bie åußjere Şaut in ben meiften Fâllen zu eimer feften, bornigen ober Ealtigen Şullle werbairtet, welche Şillle zugleich) bas (Sierift bilbet, an bem die ubrigen Srgane, be= fonders die צxuzleln, fich befeftigen. So baben alle diefe şbiere ihr SEelet gleidjam duberlich, uno es ift aljo oas Serbaltnißz zwifchen Skelet uno ben librigen Rorpertbeilen, in Bezug auf bie Riloung ber Ruckgrattbiere, ungefelyrt; nur im

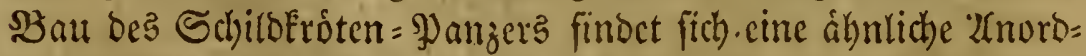

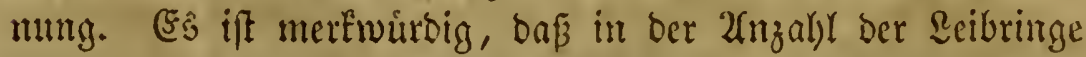

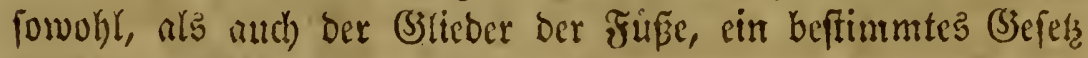




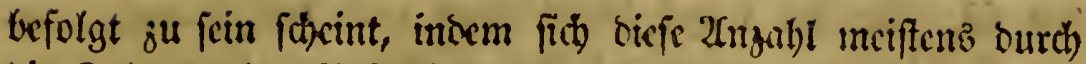

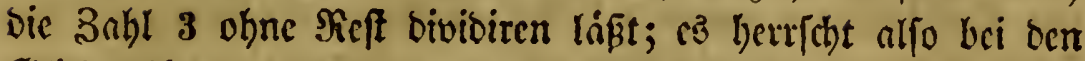
(Biticbertficten ber nnmerus ternarins. So befteht bei ben

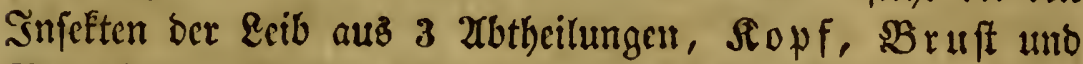

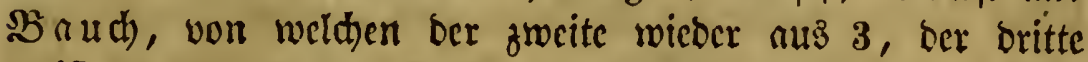
meiftens aub 6 ober 9 ßingen zullammengefest ift. 2fuch fimben fich hauffig 3 Fuppoare, uno jeber Fuf hat 6 ober 9 , niemals mehr, bod) wohl meniger, (Jiclente.

Unter ben brei afbidnitten bez $\mathfrak{L}_{\text {eibez }}$ ift ber Sopf am wenigften entwidelt. Freitid) bemerft man an ihm in ber Niegel $\mathfrak{A}$ ugen, unb jwar jufammengefegte, o. h. aus viclen

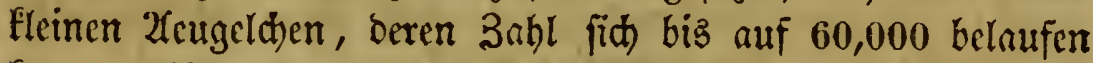
fann, gebildete; alkin beutlidhe 5 hren fehlen ben Bstieber=

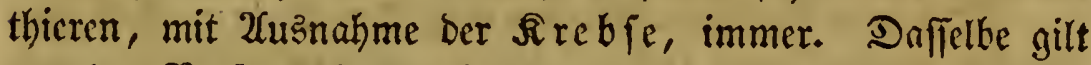
von ber $\mathfrak{R}$ afe uno ber 3 unge, als fhmedfendem Srgan. Nidyts befto weniger horen und ried)en vicle (S)liebertliere, bejonders \$nfeEten, fchr gut. Eigenthümliche Drgame bez Sopfes find bie Fúhlhórner (antennae), bald lnnge, bald furje seglieberte Fortfäke, bie mciften's auf ber Stirn jwif ben ben 2fugen frehen, und vielleidt bie Stelle bes (Jiehororgnnez vertreten; zum Fithlen uno $\mathfrak{x} a$ ften bienen fie eigentlich nicht. Manchen, wie ben Spinnen, fehlen fie. Der Mumb ber (Bstics Derthiere hat in ber Fiegel Sicfer, beren 2 nzabl aber fehr ver= fhieden ift. Die Infeften haven nur zwei, weldhe aber, wie bei allen (slicberthieren, in ber Mitte getrennt, alfo bafenformig ge= bilbet fint, und fich ničit, wie bei ben siudgrattbieren, von oben nach unten, fondern von linfs nad) rechts jangenartig gegen einander bewegen. 2An biefen hafigen Siefern hangen an ber 2 ußsenkonte gegliederte Fortfikze, bie zum $\mathfrak{x} a$ ften bienen, uno Daber $\mathfrak{I}$ after ober Freffipizen (palpi) gennunt wer= Den. Bei fehr vielen Btiederthieren find aber auch bie Site fer zu einem Saugriffel umgeffaltet, fo z. B. bei ben. $₹ l i e=$ gen, Bienen, Echmetterlingen unb $\mathfrak{B}_{\text {anjen. }}$

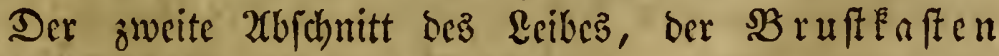
(thorax), ift hantfig mit bem Sopf verwactjen. (Fr tright

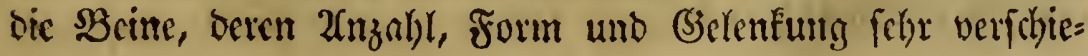


ben iff. Sn ber Regè jeboch fann man brei Scauptglieber, ben SchenEel (femur), dab S chienbein (tibia) uno ben

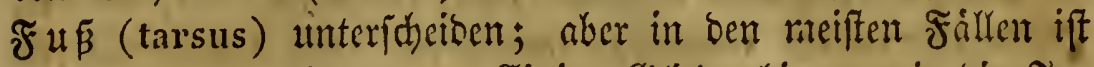
jeber wieder geglicoert. - Einige SSliederthiere, wie bie Sn= feften, haben, außer ben Beinen, noch båutige Flúgel, bie

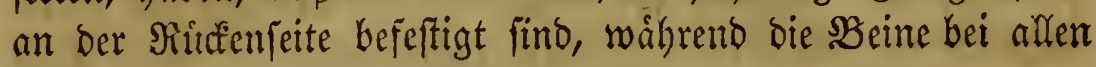
(5)ieberthieren an ber Bautchjeite fitzen.

Der britte 2(bid)nitt bes Reibes, ber Scinterleib ober $\mathfrak{B a u}$ ah (abdomen), ift bald mebr, bald weniger, bald gar nidut voin zweiten getrennt, aber von allen breicn immer am Deutlicblten geringelt. Diefer Scinterleib enthailt bie (5rnaib)= rungs = uno Fortpflanzungsorgane; audh fifken an ifm nicht felten noch Fufe ober furartige 2(nh)ainge.

Son Den Ernáhrungsorganen ift ber $D a r m$ ftets fehr beutlich, meiftens nur fo lang als ber Risrper, bismeilen brei= mal fo lang uno bríber. Sn ben meiften Fiallen hat er eine 2ffteroffnumg, in anderen felslt jie, und bann ift ber gabel= fórmige Darm wohl mit vielen blinden Fortfäten verfeken.

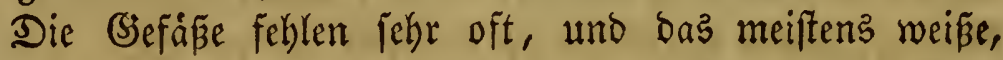
felten rothe (bei ben Rothwirmern ober 2(nnelioen) Blut fließ̄t Dann frei im Sorper umber, boch ift immer ein Scerz Da, yon welchem bie SBlutberwegung altsgeht. Die im SIS af:

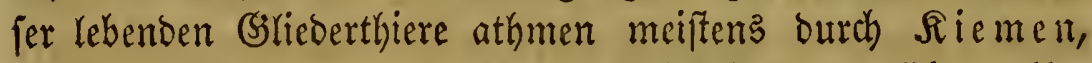
welche theils am ganjen Sirper, theils blos am Scinterleibe,

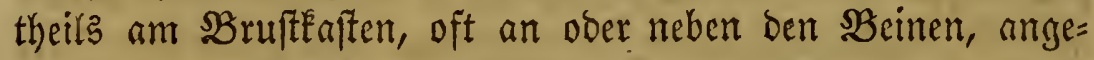
brad)t finto. Die in Der $\mathfrak{R}_{\text {uft }}$ lebenden athmen theirs burch $\mathfrak{u}$ ungen (Spinnen), theils burch vicle feine $\mathfrak{L}_{\text {uftrobren }}$ ober $\mathfrak{I} r a d$ cen, weldbe fich im ganjen Sisper baumartig verbreiten (Injêtten).

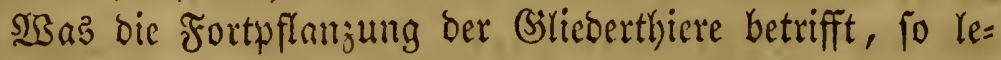
gen bei weitem bie meiften (Fier, und bic außfFriechenden Iun= gen find ben 2altern in ber (Sieftalt oft unályntich). Durch)

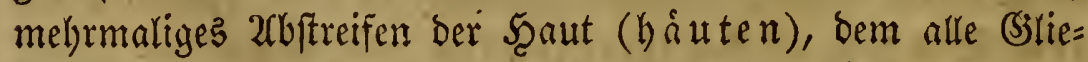
berthiere unterworfen fino, verándern fie ihre Geftalt, bis fie Den 2eltern kilneln. Dies nennt man cime Serwanolung ober Metamorphofe. 
Son ben librigen inneren Srganen liefern nod) bie $\Re e x=$ ven cin gutes fienngeid)en. Sie entipringen nelynlid von ei=

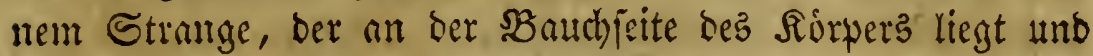
in jebem Bsliebe zu einent Snoten anjehwillt. Fin fold)es Enotiges Rerveninftem ift mur ben (jitiederthieren eigen.

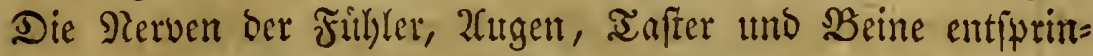
gen von ben ilynen naljegelegenen Inoten. -

Die (Eintleilung ber sitiederthiere ift folgende:

a) Snjekten. Shr \&eib ift in brei Scauptabidnitte ge= thcilt, am mittleren 3 Fuppaare.

b) 2frachniben. Sigr. Reib ift in zwei Şauptabjanitte getheilt, am erften 4 Fuspanare.

c) $\mathfrak{A} r u f t a c e e n$. Shr $\mathfrak{e} e$ b ift in viele meiftens un= gleid, Ringe getheilt, 5 oder mehr Fußspanre.

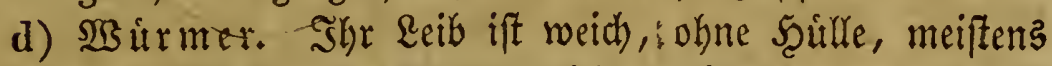
olne Fuse, und oft undeutlich gegliebert.

\section{F in $\mathrm{f}$ te $\mathbb{R} \mathfrak{i}$ a f $\mathrm{fe}$.}

\section{Snifeten oder Rerfe, Insceta.}

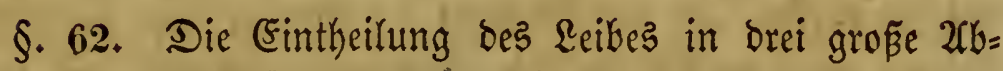

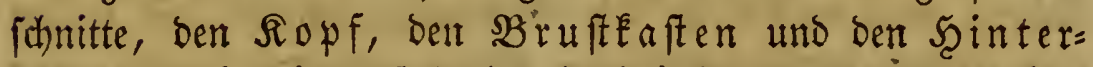
leib, fo wie bie auf iech 3 beichrantte $2\left(n_{j}\right.$ ah) ber $B$ Beine,

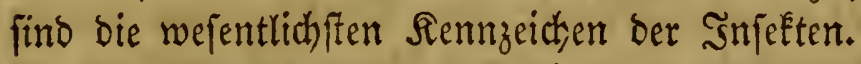

2(n Sopf fitzen nur jwei geglieberte Fithler, beren (Se)= ftalt fehr verifbieben ift. Die 2fugen find gros uno jufam= mengejeşt, boch fommen auper biefen Scauptaugen noch z wei oder brei kleme, einfache, alff-der Stim oder bem Scheitel

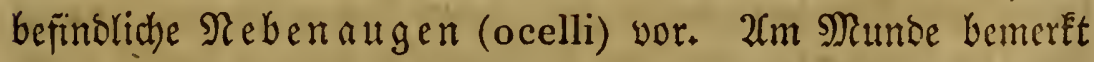
man eirte $\$$ berlippe (labrum) und jwei hafige Sberkie= fer (mandibulae), beide ohne $\mathfrak{Z}$ after, bann jwei mit $\mathfrak{I}$ iffernt verfehene Unterfiefer (maxillae) und eine eben bamit aus= geruffete Unterlippe (labium). SBisweilen finto bieje $D^{2}=$ gane zu einem fautgenoen $\Re$ itfiel umgebirbet.

Zfm $\mathfrak{B r}$ uffét aften, ber aub brei bald mebr balo weni= ger verwactjenen ßingen befteht, fitsen bie Bcime. Bon ben 


\section{Erfter âfdgnitt. B̧oologis. II. Bliedertbiete.}

brei Scauptgliebern befteht ber Sd)enfel aus brei, bas Schien= bein aus einem uno ber Fuf aus einem bis fünf (Blies benr. 2luserbem sommen nod) bautige Flingel vor, bie feit= lict) am zweiten umb britten ßinge fitsen; in ber Seaut ber: felben berbreiten fidy hornige 2roern. SBiŝnei'en fino bie vors beren ganz hornig, unb heipen bamn Flugeldeden ober Dedffilber (elytra).

Der Scinterleib beftelst aus fechs ober neun Rin= gen, beren jeber in 2 Şálften getheilt ift. Er tright Feine F̌sue, wohl aber am Enoe sorften, oder einen Stadbel, reid)er nur ben Szeibden eigen ift, und theils beim $\mathfrak{L}_{\mathrm{eg}} \mathrm{en}$ ber Eicr, theils als wanfe gebraucht wirb.

- Die Infeften athmen alle burd $\mathfrak{a} u f t r \delta h \mathfrak{e n}$, welche von ben zwijd)en je jmei Ringen in ber Serbinoungshaut

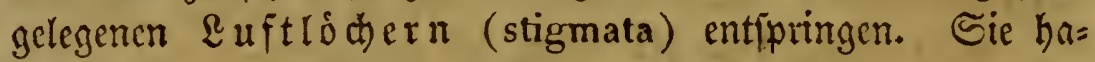
ben Keine şlutgefáp̄e, aber ein langes, mehrfammeriges, am

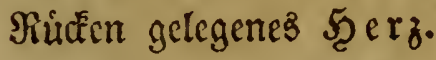

Die aus ben Eiern gefruchenen Jungen haben entweber bie (Seftalt ber Mutter, uno unteridbeiden (id) nur burd) ges ringere Grópe und ben Mangel ber Flugel; oder fie fino ben Xeltern ganz unábnlid, wie ein langer, runber, mit kurzen

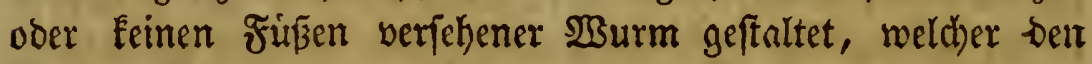
Ramen (Engerling, Raupe ober Nabe, aud Larve (larva) erthalten bat. Sene İungen báuten fich) bló̉ uno be= fommen nach unb nad) Fluggel, bicfe bagegen báuten fich auch, ofme ihre Gieftalt zu ánbern, uno liegen Darauf eime Beit lang unbeweglich ba, obne Rahrung einzunehmen, meiftens von fei= benartigen (Seweben umfchloffen; Dann führen fie ben Ramen

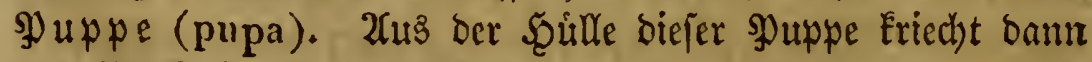
bas Sinfeft in jeiner vollfommenen Form eift hervor. Dieje

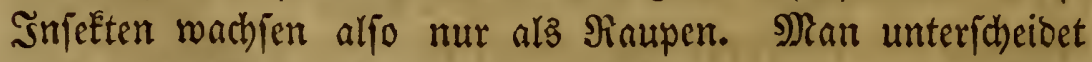
nach biejer \$eridjiebenkeit bie Infeften mit ber erfteren $B e r=$ wandlung als folche mit unvollemomener Retamor= whore, bie ber jweiten als mit vollemomener SAetamors Who le begabte, bic lesteren find bie zahlreicheren. 
Die (Struppe ber Infetten ift íbrigens unter allen abier= gruppen bie grußste; man Eennt gegenwártig gewieß 80,000 verfotichene 2(rten, bie theils won pflanglicher, theils won thie=

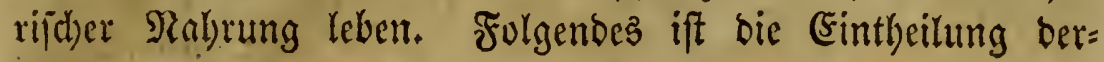
felben:

A. Infeften mit vollêommener Metamorphofe.

a. Mit beifenden Munnotheilen und Frit=
gelberÉcn.
1. Jan. Siáfer.

b. Mit faugenden Minnotbeilen. 23ier unglcide nackte Fliget. . 2. - Jmmen.

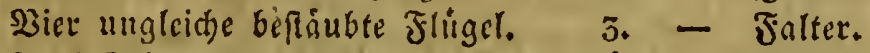

3mei Jlígel. •? • 4. - Jliegen.

B. Infeften mit unvolfémmener Meta= morphofe.

a. Mit beigenden Mundtgeilert.

Dier gleidgefiloete negförmig ges

aderte Flügel . . 5. - Nesfligler.

Bier ungleide Flüger, Die vorierent

Iederartig, ble ginteren der Lange nacif gefaltet.. .6 - Birabfigiter.

b. Dit faugenden Mundtgellen. . 7. - Sgalboecter.

Erfte Familic. Siffer, Coleoptera.

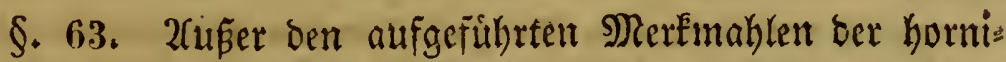

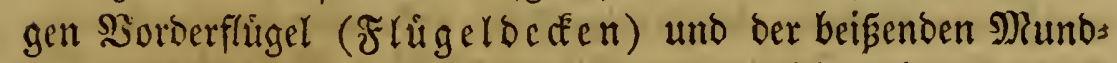
theile, haben fie noch andere cigenthumliche (Figenichaftent. So finben fich faft nientals bei ihnen Nebenaugen; ihr erfter SBruftring ift freier abgefonoert, bie beiben folgenben aber find nit bem $B$ auch) enger verwachfen uno von ben Flugeldedten verbedt. (Fin Stachel fommt ihnen nidyt zu. Shre Earven haben bơd)ffens 6 Fupe, oft gar Feine, eimen beuttlichen Sopf uno leben in ber Erbe, im Scolze, wenige auf SBlâttern; bie Soupe hat freie, fidstbare (Sslicomaken. Dahin:

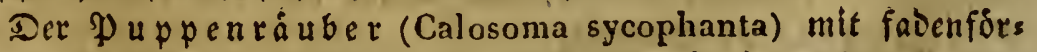

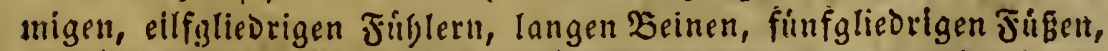

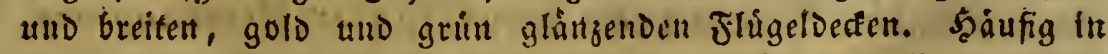
Baloungen, friłt, wie feine fdmarge Larve, 厄dimetterlingbranpen, bejonders Der Liparis dispar.

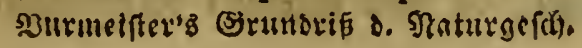




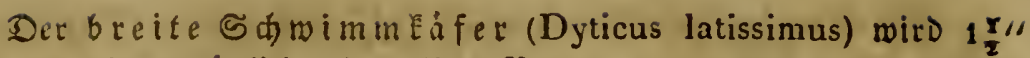
lang, ift oben grunlid) mit gelbem Rande, unten ganz gelb; Sainter: beine breit georúft, mit Saaren am Rande zum Sdmimmen. Jüb= ler fadenförmig. Nionndyen mit ermeiterten Sorverfüben, Weibden mit gefurd)ten Flingeldeden. Sebt, mie feine J" lange, grünlidgelbe Larve, in Jifकtriden, uno ftellt Dem Fifdlaid nadb. Selten.

Der To D te u gráber (Necrophorus vespillo) Gat Enopfformige, geftelte Fublergorner und Ginten abgeftute Frlugeloecken; Farbe

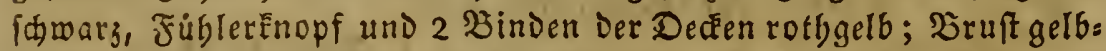
Gaarig. Die breite, famarze Larve lebt im 2las, Das Der Siafer mit feimen Sameraben eingefdarrt bat.

Der $\mathfrak{R}$ a i á fer (Melolontha vulgaris), Jübler fäđerfórmig,

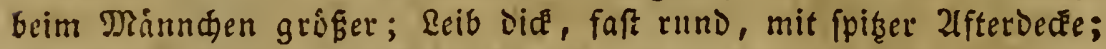

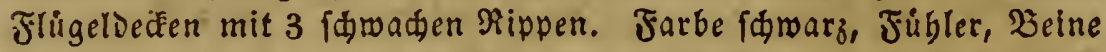
und Flügeldeden braun, Baudringe mit meifem, Dreiedfigem Seis tenfled. Die gelbe, meide, rotblespfige Rarbe (Engerling) bat feds lange Beine und lebt in Der Erde von Grasmurgeln.

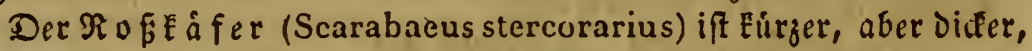
bat ágnliabe, fádberformige, aber Eleinere, bei beiden Gefhlechtern gleide Fúgler uno eine ftumpfe 2lfterdefe. Jarbe fintwarg, ftagl= blau angelaufen. Reft im pferdemifit, die Larve in Ier Erde.

Die $\{q a$ nifde Fliege (Lytta vesicatoria) bat gerade fhnur= formige Jüblgorner, und an Den vier $\mathfrak{B}$ orderbeinen fünfgliebrige, an Den binteren viergliebrige J゙̉̆be. Jarbe einfarbig metallifa grin.

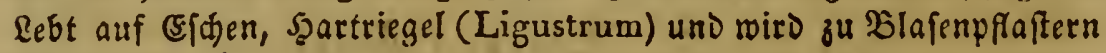
benusit.

Der S G reiner ober (B) iger (Lamia aedilis) bat lange, bors ftenformige Fubler, vierglieorige Finge uno einen jiemlid breiten, fachen fiorper. Farbe grau, mit undeutliden wolkenffecken. Die gelbe, fuflofe Larve lebt im Baafjoly; Der Siafer giebt bei Der Des rubrung einen jirpenden Ton yon fí, Den er Durd Reiben Des er: ften Bruftringes am zreiten bervorbringt.

Das Sonneneáferdien (Coccinella septem-punctata) bat cinen balbEugelformigen Qeib, Eleine in cinen Fnopf endende Fribler,

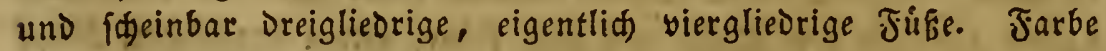

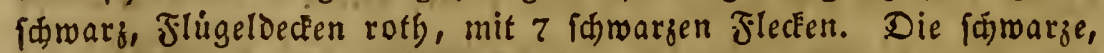
rotygeflecté, raube, fedjffifige Larbe lebt auf 'Dflangen, Die you Blattlaufen bemognt werden, und nágrt fíd yon Diefen. Daber Blattla us lóve genannt.

3weite Familie. S mmen ober Scautflügler, Hymenoptera.

§. 64. Sie baben einfacke 2augeldben und Nekaugen, vier ungleidhe, báutige Flugel, bie von wenigen verzmeigten 


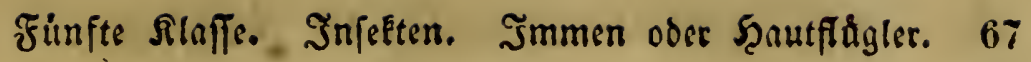

2rbern burdsjogen werben. Bisweilen feblen fie. Mundtheile faugend, bie Unterfiefer find flact, leberartig, und bilden cine Scheibe, welche bie in cine fleifhthe, oft lange, rỏkrenfórmige, auch wohl behanrte, oder in mehrere $\mathfrak{L} a p p e n$ getheilte 3unge auslaufende Unterlippe umgiebt. SBseibchen mit einem Sta=

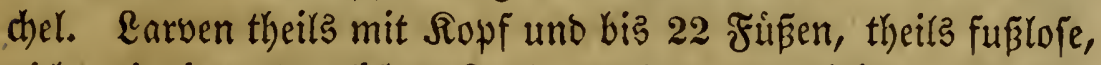
nicht mit eimem beutlichen Sopf veriekene, alfo blinde, Maben.

Die SdI upfmespen (Ichneumones), mit langen, borften= förmigen Jüblern, Diunngeffieltem Şinterleibe uno Eurzer Unterlippe. Die $\mathfrak{W} e i 6 d e n$ fteden die Sdrmetterlingzraupen an, Iegen igre (Eier in Diefelben uno tídtell fie Dadurd. Man Kennt fegr viele (gegen 1000) einfeimif̧he Zrten Diefar Ģruppe.

Die $\mathfrak{B}$ e \&pe (Vespa vulgaris), mit geEnickten Jäblern, Der Ránge naw gefalteten Dberflủgeln, unt Kegelförmigem, geftieltem

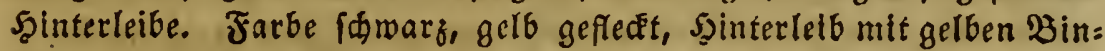
Den, Deren iede 2 fomarze Punkte fúbrt. Das 2 Beibden bauet ein

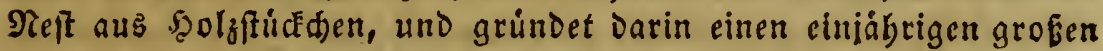

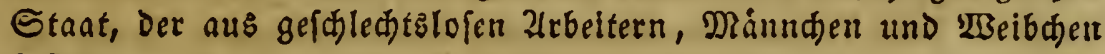
beftelt.

Die Şonigb tene (Apis mellifica) hat gennifte Fäbler, eine tange, tunie Bunge, ungefaltete DGerflingel uno brcifgedricfte Sin:

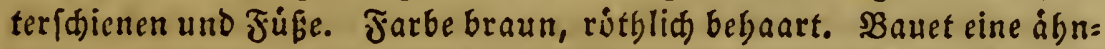

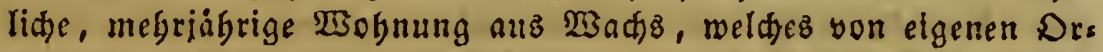
ganen am Ssinterleibe abgefondert mirb; fammelt Blumentonig ein jur Ennágrung Dez Etaates, Der aub vielen gefdlect) tslofen $2 \mathrm{lr}$ : beitern, vielen Daannden (Drolynen und einem $\mathfrak{B e i b d e n ~ ( R i u n t g i n , ~}$ siseifel) beftebt.

Die $\mathfrak{A}$ meifen (Formicae) baben geenidéte, kolbige Júbler, Eeine verlángerte 3unge, und einen runden, yon einem Enotenfórmi= gen oder Sthuppenformigen Stiel getragenen Şinterletb. Mande

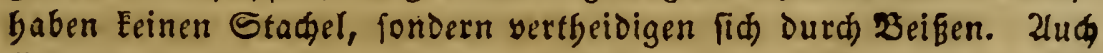
fie leben in Solonien, aber in Der Erde. Seber Staat befiegt aus ungefligelten 2rbeitern, geflügelter, gríferen $23 e i b d)$ und Eleines ren Diännden. Die fogenannten $\mathfrak{A}$ m ei f e ll e ie r find die \$uppen. -

Dritte Familie. Falter oder Sd) metterlinge, Lepidoptera.

\$. 65. Sie haben vier baiutige, von fleinen badjoiegel= artig itber einander liegenden Schuppen bedectte Flugel, beren binteres şaar Eleiner ift, als bas vorbere. Munotheile faus geno; bie meift langen, hublen, fabenformigen Unterfiefer bils 


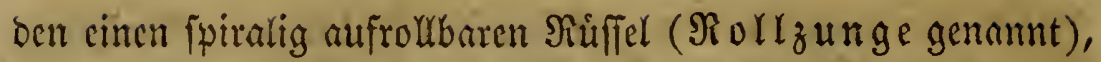
ocr von ben $\mathfrak{x}$ aftern ber Unterlippe verbecft wirb. Enrven $\mathfrak{l}_{\mathfrak{R}}=$ ben frei auf SBlittern uno haben 10-18 Fuße. Souppe mit verfteften Giliciern, oft in (Geppinnften eingeichlofien.

- Die Tagfalter (Papiliones) baben lange, Eulbige Fübler,

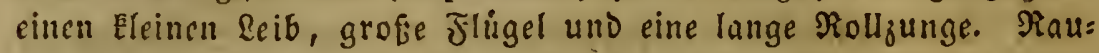
pen báufig mit Dornen, Puppe frei hángend. Der grof́ce(P.po-

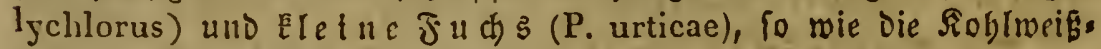
linge (P. crataegi, P. brassicae, P. napi) gegoren Gierber und fino unz ZIHen beEannt genug.

Die Samármer, Ubend= ober Dámmerung f falter (Sphinges) baben einen Didén, Eegelfsrmigen Eeib, Eleinere, fdimale

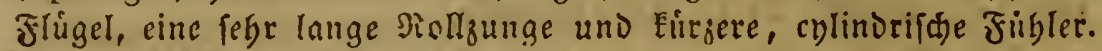

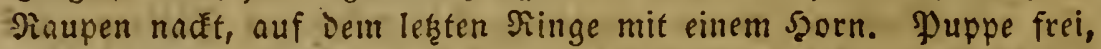

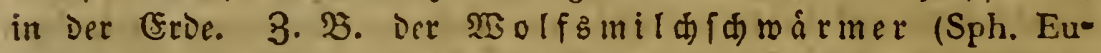
phorbiae), Der TodtenEpf (Sph. atropos) und viele andere.

Die $\mathfrak{N a d t f a l t e r ~ o d e r ~ S p i n n e r ~ ( B o m b y c e s ) ~ G a b e n ~ e i n e r z ~}$

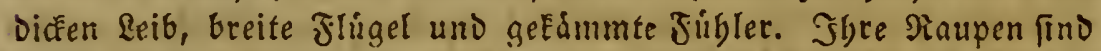
meiftens ftarE bebaart uno fpinnent ein Didgtes Berwebe. 3. 23. Der Seidenfpiner (B. mori), Die ringelmotte (B. neustria),

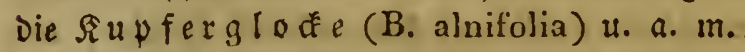

Die \&ulen (Noctuae) baben borfenfirmige Jübler, Eegelfirt: nnige Reiber, unD Eleinere F́lugel, Die Den Reib wie ein Dad) beiefs: Een. Raupen leidt begaart oder nackt; verpuppen fid in einem

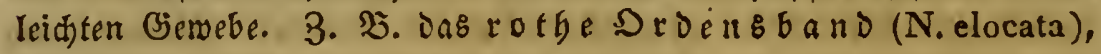
Die $\gamma$ (E ule (N. gamma) u. s. a.

Die $\mathfrak{B}$ lattwi deler (Tortrices) Gaben borftige Jühler, breite, abgeftufite oder am Ende ausgefdiveifte Jlinget, Die flad auf Dem Reibe liegen, uno einen meiftens nur Eleinen Sírper. SGre nadten

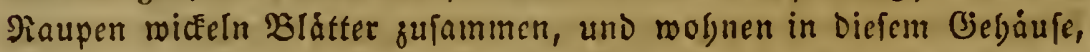
ober bobren Fridte an; f. D. Die Doft mutte (T. pomana), Dez

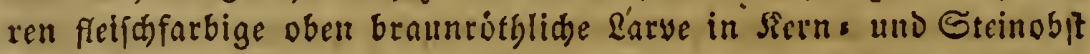
Gänge bolirt.

Die Rotten (Tineae) baben feine, borftenformige, oft febr lange Fübler, längere weiter bervorftebende Tafter, und fammale, flad neben einander liegende Jíngel; 8 . $\mathfrak{b}$. Die Sireider motte

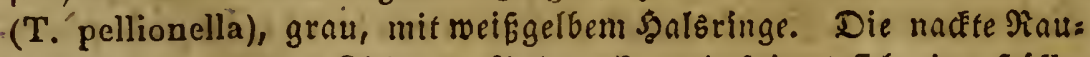
pe lebt in mollenen Sileidern, SelowerE, und fpinnt fíd eine Scúlle, worin fie beftándig fecét.

Sierte Familie. Fliegen Doer 3weiflugler, Diptera.

§. 66. Sie haben faugente Munbtheile; b. b. einen 


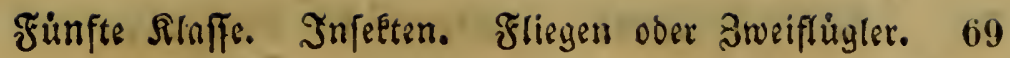

meift Enieformig gebogenen, weichen Riffer, in welchem barte Borften Yiegen. Scinterfliigel fehlen, ftatt beren zwei geftielte Sinopfchen (S chwinglolben, balteres); bie vorberen bimn= háutig, nit wenigen, verzmeigten, meift paralkelen ß̊ippen. Weibchen ohne Stachel, aber manche ftechen mit dem গiuffer. Earve fur = und kopflos (Made), lebt verftect; Poupe meift

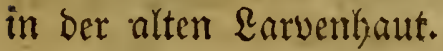

Die ád) ten Fliegen (Muscae) baben oreigliedrige Fribler uno wenige (4) Sauptabern im Flugel. Mraden leben in Mift, 2las und Pranjentfeilen; f. $\mathfrak{Z}$. Die Sd) meiffliege (Musca vomitoria), blaugrau, mit ftablblauem sointerteibe. Regt igre Eier auf faules Jleifd. Die Maden find in 7 Tagen ermadjen. Die Fre if

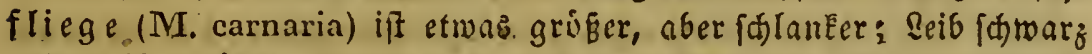
und weî́ getâfelt, Zlugen rotb. Bebiert lebenoige Maden, Die fie an's Fleifd) legt. Die Etub enfliege (M. domestica), ein Drit: tel fo großs, grauldwar Made lebt im Mift.

Die Stedfliege (Stomoxys calcitrans) bat Das 2 nfebn ei= ner Efubenfliege, ift jebed etwas Eleiner und bat einen Dinnen,

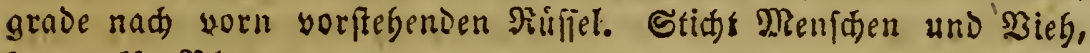
faugt alfo Blut.

Dis Że mifengat tung (Oestrus) Gat febr Eleine, Enopffors mige Jriller, und eine unfdecinbare, Eaum fidtbare Mundiffnung. Reib meiftens didft bebaart. Die Rarven leben im Reibe Der Pferde

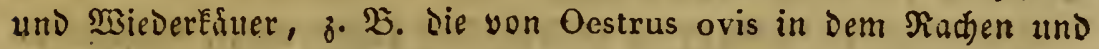

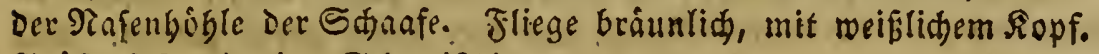
Brifbe faft wie eine Sdmeiffliege.

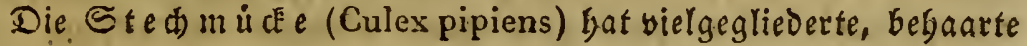
Fiubler, einen langen, grade herborftegenden Rúffel, leiăt behaarte Fluget und lange, Diume beine. Saugt $\mathfrak{B l u t .}$ Die Rarven uno

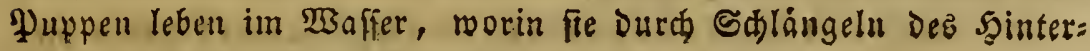
ปeibes. munter umberfdmimmen.

\section{Finfte Framilie. (3)itterflingler, Neuroptera.}

§. 67. Sie baben beikende Mundtheile, wie bie Rafer,

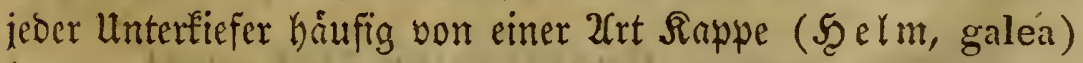
beocrit. Fiffler batb langer, faben = oder folbenformig, balo burftenformig. SBier gleich) großße, håutige, von gitterformigen

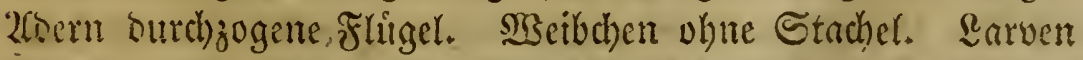

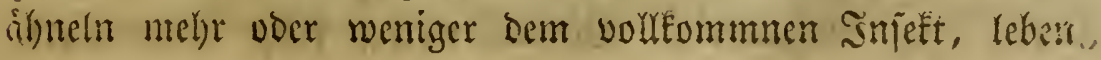




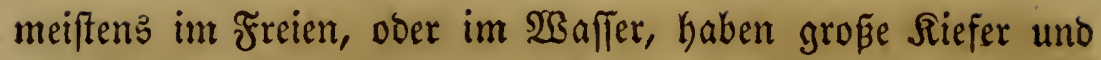
fechs Beine. Einige ruben als Şuppe, andere bewegen fich und freflen.

Der 2(meifentione (Myrmecoleon formicarum) bat Eurze, Eolbige Fubler, eine Eleine Bruft, aber einen langen Dünnen Szinter= leib. Die Eurze, breite, eiformige, etwas fladhe, mit 2 grofien fie: fern verfebene Earwe grábt Tridter in Sande, und nábrt fíd vou Den bineinfallenden Snfeften, bifoniers 2lmeifen.

Die Florfliege (Hemerobius perla) bat lange, borftenfirmige Fubler, fonft Den Sau Des Borigen. Shre Farbe ift ein blafies Griun, Die 2lugen golden. In Biarten. Die DunEelfeifdrothe, lan: zettformige Larve lebt zrwifhen Den Liattláuen auf Splanzen, und frift lie mit ibeen grofen, weit vorftebenden Siefern.

Die SE orpionsfliege (Panorpa communis) bat ein fona: belformig verlängertes Maul und borftenfirmige J̈hibler; Der Jjin: terleib Des Mảnndens låuft in eine Dicke, an zmeien B̧liebern bảns gente 3ange aus. Farbe gelblid, braun geflect; Fligel ebenfo. 3ange rotl. (jemein in Gebuld)cn. Sarve nod unbefannt.

Die Fribling f fliegen (Phryganeae) babenlange, borften= fórnige Jilbler, ein farwades bebis uno vicr ungleide, beftáubte oder behaarte Jingel. 3. 2. Die grofie $\mathfrak{F}$. (Phr. grandis), getb= grau, mit vielen bunfilen, gralten uno meifien, vermorrenen Zeids: nungen auf Den Dberfligeln. Die unter Dem Ramen Sprodé n beÉanten Rarven leben in Maffer, atgmen Durd Siemen, und bauen fich Pigren, in Denen fie wohnen.

Die $\mathfrak{i}$ a flerjunģ $\mathfrak{e} \mathfrak{x}$ n (Libellulae) baben Eurze, borftenfor: mige zurler, cin ftarfes (jebif, uno vier gleid) grofie Fligel. Die

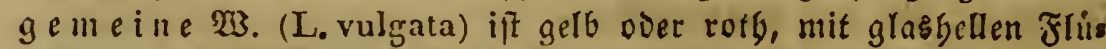
geln, Die am Grunde breit gelb find. Bemein auf $\mathfrak{B i e f e n . ~ D i e ~}$ Earven leben im sisaffer, min baben eine ansftredebate Zange am Diaul zum Ergreifen Der Beute.

Dir ga afte vocr Eintags fliegen (Ephemerae) Gaben Dic= fulben Fubler, ein fatwades (Sebif uno vier ungleide oder nur jwei Flingel. Sainterleib mit 2 oder 3 (Enoborften. Die geneine 5 . (E. vulgata) ift gelblid, braun geflect, Jiligel brain geiprenfelt.

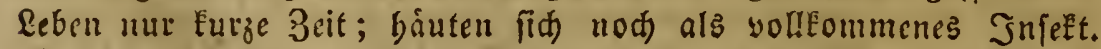

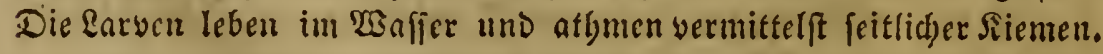

Sectiste Familie. (sirabflugler, Orthoptera.

§. 68. Sie baben ftarke, beifende Nundtheile, bei wel= d)en die Unterfiefer von cintem biden Şelm bebedt fino. Fubler lang, vielgliebrig. \$Jorberbruftring freier abgefonbert. 
Fủnfte Sinffe. Infeften. Girnofluggter. Şatbosker. 71

Flügel grabe auß̊geftrect', bie oberen leberartig (Ded én), bie unteren ber Lange nach gefaltet, nur beim Shrwurm umge= fd)lagen. Sieine volffommene Sierwandhng. SSeibchen mit jweiflappiger $\mathfrak{L}_{\mathrm{egeidheide.}}$

Der $\mathcal{O h r w}$ urm (Forficula auricularia) bat perlfdnurformige

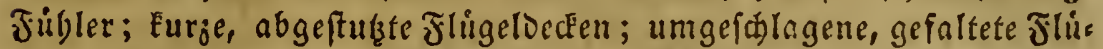
gel, und eine Zange am ๔dwangrnde. Riricdt gern in Eleine Róther; frifft faftige Frudete.

Die Grolllen (Grylli) baben lange, borftenfórmige Fübler, Dreiglieorige Jüse und verlángerte, frárkere, bintere Springbeine. Die Jelogrylle (G. campestris) ift Duneelbraun, mit blutrothem Snnenrande Der ŞinterfhenEer. Semein in Erolidgern auf FerDern; Das Máunden giebt, wie Das Der folgenden Zlrt, einen Iauten zilt: penoen Ton yon jït. Das Szeimđen (G, domesticus), Eleiner, einfarbig gelbgrau. Sn Şáufern an warmen Stellen, befonders bei $\mathfrak{E}$ dickern.

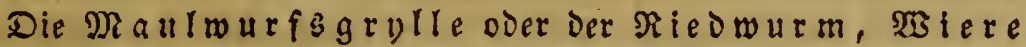
(Gryllotalpa vulgaris), bat Eeine binteren Epringfübe, aber breite, vordere Daulmurfşfúpe zum Brabell. Zluf Jeldern in Erolodern.

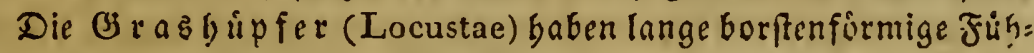
Ier, vierglieorige Jǘfe, uno verlángerte, 'bintere Epringbeine mit

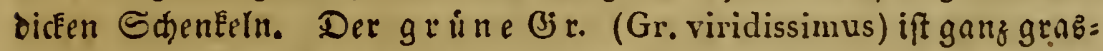

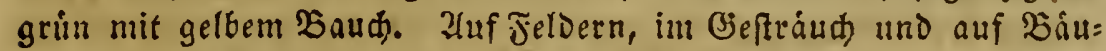
men. Das Mianden giebt einen lauten jirpenden Ton yon fich.

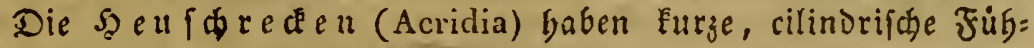

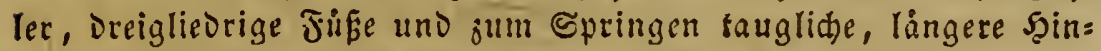
terbeine. Berridtigt ift Die $3 \mathfrak{u}$ ghe uf wegen ibrer verbecrenden 3úge. Sie wird $1^{\frac{\pi}{2}}$ lang, iff famukig grün, mit braun geffeckten Decken. Ueberall, in Deutidland, aber meiftens nur einjeln.

\section{Siebente Frmilie. Scalboecker, Hemiptera.}

§. 69. Shre Mundtheile find in einen Sinnabel umge= wandelt, ber aus ciner geglieberten Scheide uno vier barin liegenoen Borften befteht. Sorberbruftring freier abgefonoert: Flingel ungleich, bie vorderen håufig zur Scâlfte hornig, bic hinteren mit verzmeigten 2roern; biefe oft ber $\mathfrak{l}$ ange nad ge=

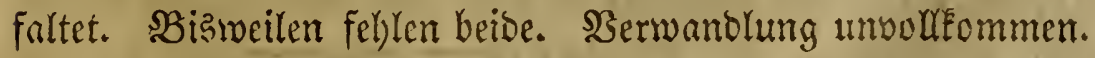

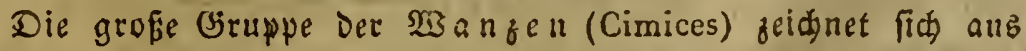
Durd 4-5 : gliedrige, meiftens lange Júblew, cine $3-4=$ gliedrige

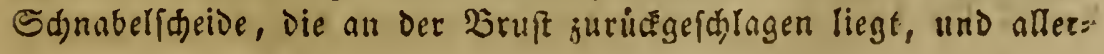


meiftens balb bornige Flugeldeden. Dei ber $\mathfrak{B}$ et twa a n f e (Cimex lectularius) feblen Die Flügel, oie Ednabelfcheide ift oreigliedrig,

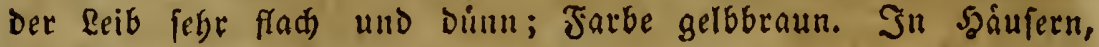
Bettftellen, fangt Menichenblut.

Die 3irpen (Cicadae) baben Eurge, borftenformige Fubler unb einen Dickeren, Eurzeren Reib. Borberfligel nid)t abweichend, oder nur lederartig. Die Singzirpe oder (SiEade (C. orni) wird cinen 30 ll lang, ift braun, mit gelben Streifen au baudh.

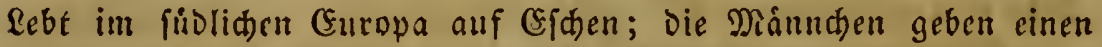
lautelt, jirpenden Ton von fid). Die $\mathbb{S}$ a a um irpe (C. spumaria) bat einen fpiferen, Dreifeitigen Sopf, TeDerartige \$orberfligel und bintere Springbeine. Farbe grau, mit 2 braunen Sinden. 4"' lang. Die grime Larve lebt auf \$franzen, und verbreitet einen

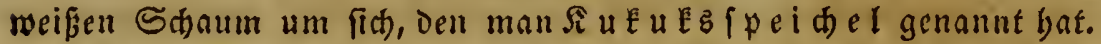

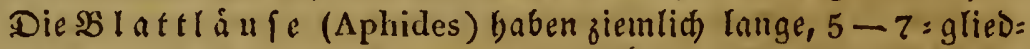
rige Jibler uno vier ungleide, bantige Jlingel. 2lmi Baud baben fie binten 2 Riblen, Die einen bonigartigen Saft aubtropfeln lafien. Sie leben auf Pfanzen, fungen Deren Saft, und verurfachen Da:

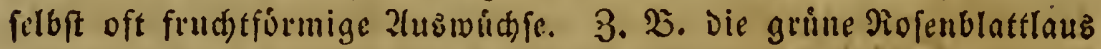
(A. rosae), an Den iungen Trieben Der Rofe.

Die eige ut $t$ ide $\mathfrak{n} \&$ á ufe (Pediculi) baben furze, $5=$ glied: rige Fubler, Eeine Jlügrl, uno Gakenformige, cingliesrige, zum Jefts flammern eingerid)tete Finge. Dic Irten leben nuf Sängetbieren und faugen Deren Blut. 3. S. Die Si p fla pitis), gelblid), mit braun Durdfdeinendem MRagen, $1 \frac{3}{2} "$ lang.

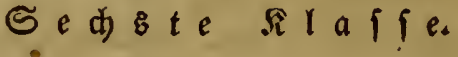 \\ Spinnenartige (sliebertbiere, Arachnoda.}

§. 70. Die (Fintheilung bes Siorpers in z wei Şaupt= abichnitte, won welchen ber vorbere die Simnesorgane unb bie

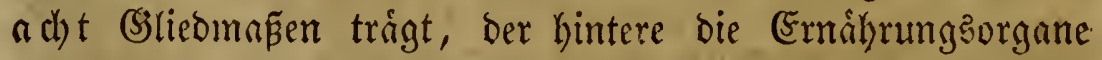
enthalt, giebt ben Sharafter biefer (siupwe. Sigre Munotheile befteben, wie bei ben Injelten, ats jwei meiftens jeberen= ober bafenformigen Sberfiefern, jweien fleineren mit groß̧en כaftern verfebenen Unterfiefern uno einer unbeutlicken tafter= lofen Unterlippe. Die Fithlhisner feblen, ebenjo bie ju= fammengejetzten 2ugen; bie Aujahl der einfadjen wechielt von 2 bis 12. Die Safter Der Unterfiefer fino oft gró̈ uno 
fheerenformig, bie Beine nie. Şbre 2(nzal)! ift immer a cht $t^{*}$ ). Der Sinterleib ift entweder ein einfacher ungeglieberter sBauch (debte Spinnen), ober er befteht aus -mebreren, Deut= lichen ober unteutlicken (Siliedern.

Bon inneren Srganen zeigen bie 2(th)mungäwerk jeuge Serichiedenkeiten. SBet den einen (id)ten Spinnen) fino ez

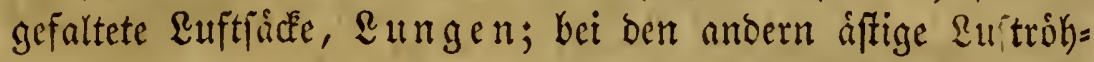
ren, Irackeen; bieje haben mehrere $\mathbb{Q}_{u f t}$ tocher, jente nur zwei, bie in beiben Fillen am Scinterleibe liegen.

Die arbiere biejer (Struppe bauten fich, uno verwandeln

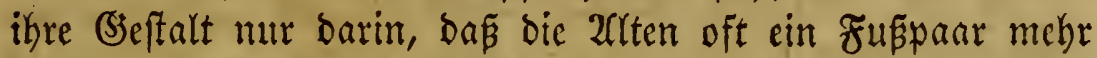
baben, als bie Jungen. Sie freffen faft nur thierifothe Rah= rung und befizen Giftorifen, uno oft ftark verlesento Stachel. Mran theilt fie in 4 (siruppen.

a. Spinnen mit \&uftiåafen.

1. Baud) Deutlid getrennt, aber uns geglievert. . . 1. Jam. 2ledfete Spinnen.

2. Daud undeutlid) getrennt, ges gliebert. $\quad: \quad . \quad 2$. - Storpione.

b. Spinnen mit Euftrohren.

1. Baud gegliedert, yon Der $\mathfrak{B r u f t}$ fdwadh getrent. . . 4. - \$Jgalangien,

2. Baud und $\mathfrak{c r u f t}$ gar nidjt ges trennt. $. \quad . \quad 3 .-$ MRitben.

Erfte Familie. Uedhte Spinnen, Araneae.

§. 71. Zafter und Riefer nientals jheerenförmig, bie leke= teren mit eimem beweglithen burchbohrten 3abn, in welchen Der 2fusgang ber (siftorije mumbet. Sainterteib ungegliebert, blafig; all feiner Spif̧e mit mehreten, feibenartigen Fäben ab=

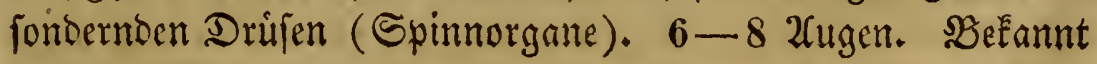
fint:

Die $\mathfrak{B}$ uf bogenem Rieferzahn und Diden, fark begaarten Dieinen. Der Bauld allein Gat Die Bríf̧e eines Taubeneirs, Die beine gegen $3^{\prime \prime}$ lang, Jarbe braun. Braftlien; faugt jungen $\mathfrak{B i g g e l n}$ Daz $\mathfrak{B l u t}$ aus.

Die Sireubipinne (Epeira diadema) gat einmártz gebogene

*) Die fectbbeinigen Nilben find nur Junge. 
Sieferbafen und aht in grwel bogenformigen Duerlinien geftellte 2(us: gen. Baud wie cine Spafelnufi, gelb ober grau, mit weiffen ein Sireuz bilbcnden Jleçen. B̈emein in Bjärten, fpint ein grofies,

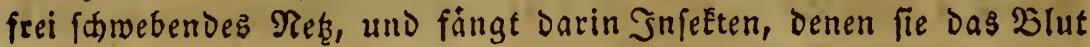
aนรfaugt.

Die Tarantel (Lycosa tarantula), faft Dreimal fo grof, Grảut= lid, Bruptftick mit einem RuckenEiel; acht 2lugen, in Der Jormeines

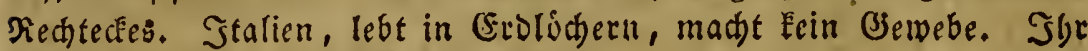
Siff erregt Ëntzindung.

Sweite Familie. SÉorpione, Arthrogastra.

§. 72. (5? fino grope 2frachnioen, mit langen meift itheerenformigen $\mathfrak{x} a \mid f t e n$ an ben Unterkiefern, bie Unkunbigen firr Beine gelten, Daher man fie für jehnfübig halten fonnte. Der Şinterteib ift ziemrid mit bem Bruffftite ver= watbjen, aber in methere (oft 12) Gslieder getheilt.

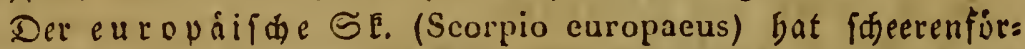

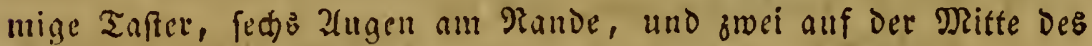
Senbalothorar; oie 5 Teţten Glicder des Sainterleibes find viel Dinn= ner uno lánger, bilden einen Eđhwanj, Deffen Spiţe eine Blafe trágt, weldje in einen gebogetn' Stadel ausläuft, Der als Waffe Dient; in Der Blafe Die Biftorife. Sild: Europa. Seine Etidje cr:

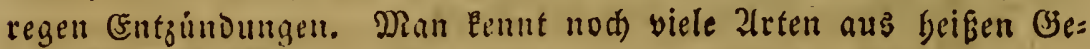
genden, Deren Stide tiotlidh fein folden.

Dritte Fámilie. Sুłhalangicn, Phalangodea.

§. 73. Sie baben fheerenformige Riefer, und lange,

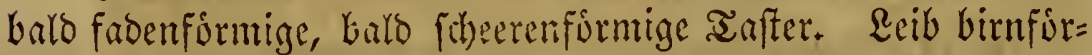
mig, gegliebert. Nur 2 2lugen mitten auf bem $B$ rufffticf. STierker:

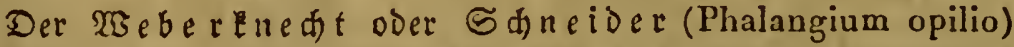
bat fheerenförmige DoerEicfer uno Tafter, einen Eurzen, Dicken Şin= terleib und febr lange, dinne beine, Die ausgeriffen nod) Iange zuceen, und fĭh, wie bei allen Spinnen, wieder neu am Siórper erzeus

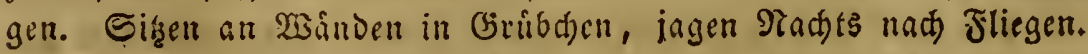

Sierte Framilie. Mirben, Acarina.

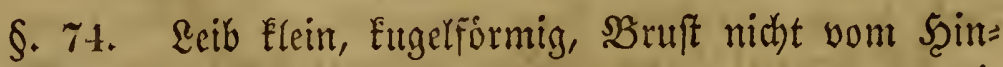

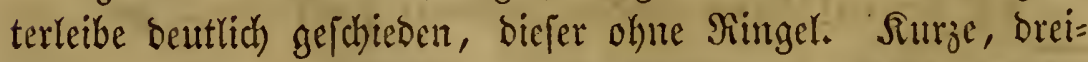
gliebrige anfter. Siefer theits borftenformig, theils lamjett= 
artig, theits foherenformig. 2-9 2 ugen. $\mathfrak{E}$ ben im $\mathfrak{Z}$ affer,

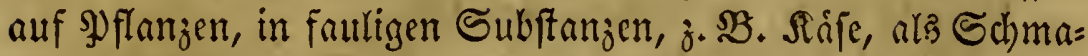
roker auf abieren, uno finto oft ungehcuer háufig. 3. $B$.

Die rotbe ejarten mitbe (Trombidium holosericeum), fhar= radjroth, fanmetartig glánzcho; Die vier vorderen beine yon den binteren entfernt. Siried)t in Gárten an fdattigen Stellen unter Wirfden umber.

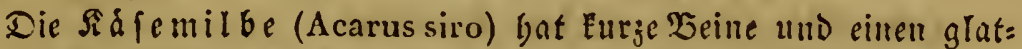

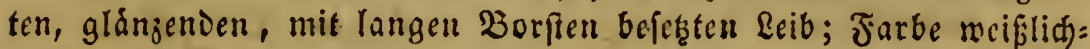
gelb. Şáufig im alten Riâfe.

- Die siaffermilben (Hydrarachnae) baben vier lange, mit

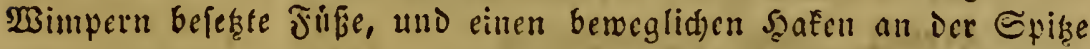
Der Dreigliedrlgen Tapter. Diefe Gilden jugleid Die Edfeide Der bor: ftenfürmigen, eines \&dnabel bildenien Silefer. Siele, rotbe und blaue,

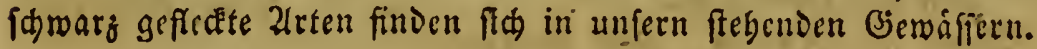

\section{Siebcnte $\mathfrak{f} \mathfrak{l}$ a ffe.}

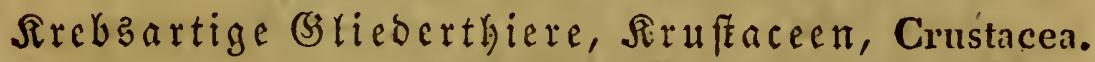

§. 75. Dieje (Sruppe ift, mit ber folgenton, bie vielge=

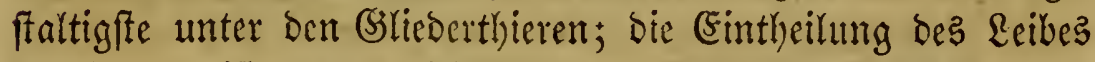
in viele, meiftens ungleiche Siinge, won welchen alle, ober bie meiften, mit (stieomaß̃en verfeken fint, beren 3ahl nicht un= ter 10 fállt, giebt noch ben beften (Sharafter. Sie baben in ber Micgel vier, jeltener jwei, Flibler; zulammengeferte oder cinfache 2(ugen, nie beite zugleich); zwei ober mekrere Niefer= pare, die oft wie Gruße geftaltet, mitunter bie \$Beine ferbft fino, uno in ben meiften Fitlen Infter tragen. Die Beine, fowohl an Bahl, als alth an (Sirofse uno Bseftalt unter fich jehr verfejiesen, enten baufig fdjeerenformig. Sie Yeben mei= ftens im 23 affer, athmen baher gewohnlich durdh Siemen, bie an ben Finsen, meiftens oben am Sd)enlel, angebrad)t fint, und eine bratt = ober bujfachelformige Bjeftalt haben; bei Eini= gen bilden fie Sicfe unter bem Scinterleibe. - Man Eann fie folgendermapen eintheiten:

I. $\mathfrak{E}$ cib in gleid)geftaltete $\Re$ inge getheilt, ber Sopf frei. Brufffaften gegliedert.

Diebr als viergebn Juffe . . 1. Jan. Metiopoben. 
Bierzeljn gleide Jüre. , . 2. Jam. Jlopoden.

sierzefn Fübe, die yorberen yon den

Ginteren veridieden. - . $3 .-2(m p h i p o d e n$.

II. Leib in ungleiche Singe getheilt, SBruftringe von einem Sanzer bedecft, Füre verjchieden.

Mehr als zeln Jifie, Sopf frei. 4. - Stomatopoden. 3egn Finke, Fopf mit Dem sruftftic vermadfer. . . . 5. - Dekapoden.

III. Reib von 1, 2 ober mefreren gro:

Ben Shalen ganz ober zum İbeil

bedect. Funpe gleichformig.

Draul obne Riefer, zwifhen Den Sdicns

Eeln Der rebn Fúfe. . . 6. - \$ócilopoben.

Maul mit Fiefern, viele blattartige

Frifie. . . . . . 7. - Pgullopoden.

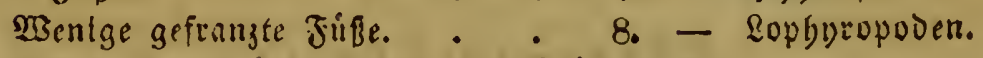

Seds Paare gefpaltener rankenfir: miger Fifie. . . . . 9. - Sirripedien. Maul (d) nbelformig mit borfenartis gin Silefern, oder Eieferlos. - 10. - Sdmaroherkebfe.

Erfte Familis.

§. 76. Shr $\mathfrak{E}$ eib befteht aus vielen $(12-50)$ volrem= men glcid)en ßingen, beren jeder ein ober jwei fußspare

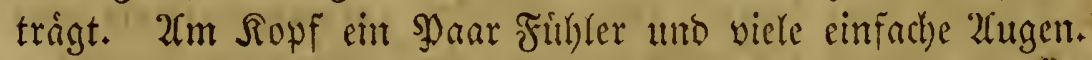

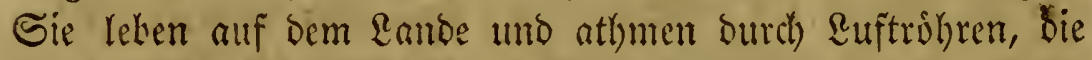

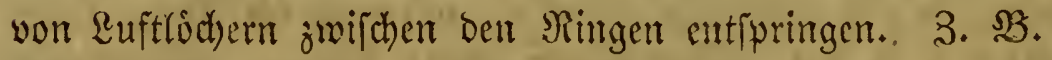

Der SEolopenoer (Scolopendra forficata), mit viclglieoria gen, fadenfórmigen Fublern, Taftern an Den UnterEiefern, 15 Juf: paaren, und abwedfelno grofferen und Eleineren Tingen. Leib flad) georúckt. Farbe Eaffebraun. Unter Steinen, Baaumrinden; gemein.

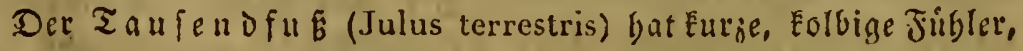
Eine Tafter, einen runden seib mit gleid grofen S̈liedern, Deten je: Deษ 2 Fufpaare trigt. Farbe braun, mit gełblidem Rúckenfteif; $1 \frac{3}{2}$ "lang, gemein unter Stcinen; rollt fid bei Der Derubrung fpiz ralformig zufummen.

3weite Fantilie. (3) feich fúper, Isopoda.

§. 77. Sht Leib beftebt, auser bem Soppf, aus fieben 
Siebente Sthrfe. Stuffaceen. 2umphipoden. Stcmatopoden. 77

Scauptringen, auf weld)e $3-6$ Fleinere Ringe, sie ben Şin= terleib (ভd)wanj) biloen, folgen; yon ben fieben SBruftrin= gen hat jeoer cill Fußpant. SSier Fithler am Sopf, viele

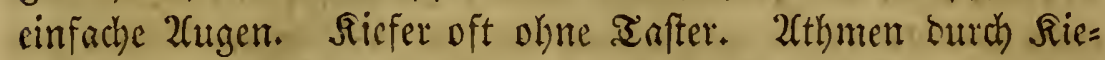
men, bie ałs sBláschen unter Dem Scinterleibe Kiegen. Die meiften Keven im SMecr. 3. $\mathfrak{B}$.

Die fielleraffel (Oniscus murarius), mit a fif glieorigen änffes ren, uno z we iglicorigen inneren Jiblerm, Reib eiftrmig, unten fract, oben gewólbt, fhbarjgrau, mit 2 Peiben gelber Jlectert. Gies mein an Miauerin, unfer Eteincu, ferbịt in feudten Siammern :c. . .

Die $\mathfrak{3}$ affer affel (Asellus aquaticus), mit lángeren Jüblern uno einglievrigem, runolidjem Sainterleibe, woran jmei geglieierte,

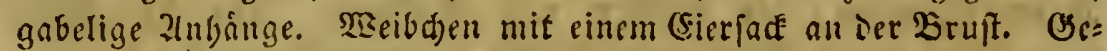
mein in fúfen B̧cwáfferr.

\section{Dritte Familie. Flobetebje, Amphipoda.}

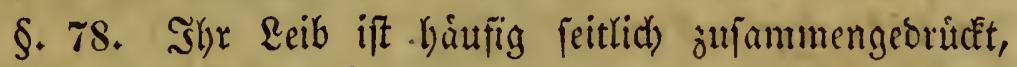
hat einen freien. Sopf, nit vier meift linngen, vielgliebrigen Filly)=

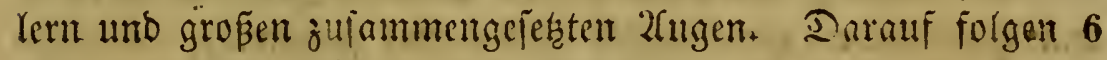

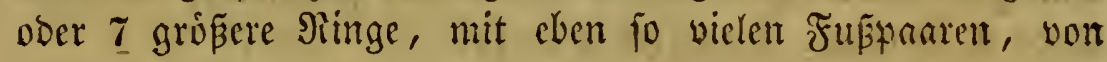
welchen bie vorberen fdeceren = voer hafenformig geftaltet fint ; an ber $\mathfrak{B a f i s}$ diejer Füpe bic Siemenblajen. Scinterlcib 3-6:

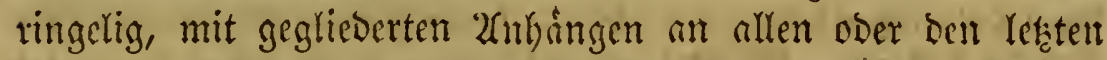

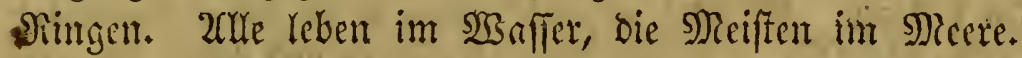

- Die Jlubgaruele (Gammarus pulex) Gat range, borfenfór. mige, obere Fúbler, Die am Dritten Blfieve mit einem zmeiten, ganz Eleinen geglieverten Jortiat verfeben fino; Die Drei erften Jufpaare

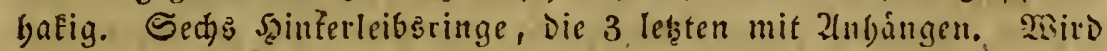
$\frac{3}{4}$ " lang, bat eine graugrinlide Jarbe, und finbet fid baufig in $\mathfrak{b a ́}$ den, f. b. bei berlin im Edaafgraber.

Sierte Familie. Maulfífer, Stomatopoda.

§. 79. Shr Sopf ift, wie bei ben \$origen, nodh frei, aber bie 2fugen fins ichon, wie bei ben Forgenben, geftielt unb

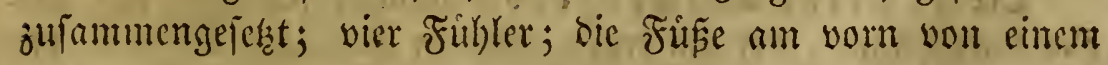
gemeinjamen \$Janjer bevedten, binten nuch gegficberten \$ruft: ftitef, bie Sienten unter bem jiemlich langen Scinterteibe, am Enbe beflelben breite Flofien. Qeben nur in Meere. 
Der $\mathfrak{S}$ e uf geu und a d $t$ Fufpaare, you melden die fünerften am Borderen, yom Pallzer bedecten Theile des Brufteaftens in ber Niabe des Mundes fifen uno bakenformig enden; Die brei binteren, kérgeren einen gegliederten 2lngang baben, und al Drei freien ßingen Des Drufteaftens befeftigt find. Der lange \$̧interleib hat oben fün crba= bene Reiften. Der firebs miro 8-10" lang und findet jid im Mittelmeer.

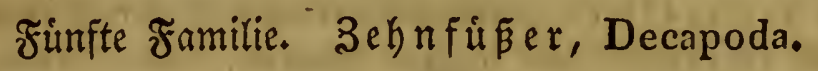

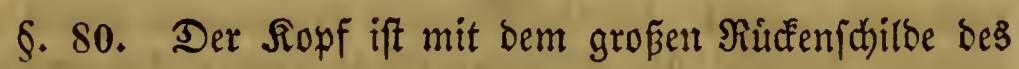
Brufteaftens vermadjen; an ihm 2 geftielte, zulammengejelste 2fugen und vier Fúhler. $2(m$ Mtumbe find jecbs \$aare von

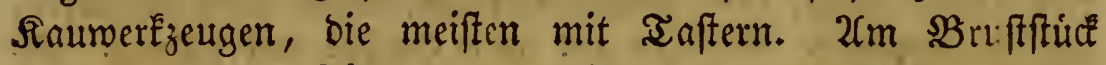

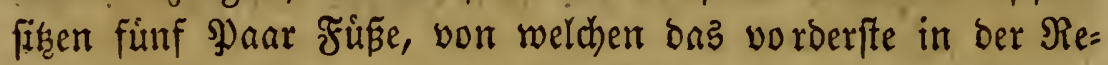
gel wie eine Scheere endet. Die Riemen fitsen an ben Schen= Felw, unter bem SBruftichilde. Der fúnfringelige Scinterleib hat feine oder verfúmmerte Fípe.

A. Mit grofem, im $\mathfrak{e} \mathfrak{e b e n}$ meiftens grabe ausgeftred: tem Scinterleibe (gemeiniglid) $S_{d y}$ wanz genannt), an beffen

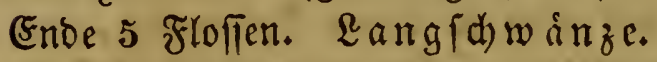

Der $\mathfrak{F} \mathfrak{I}$ u $\tilde{B} E \mathfrak{E} \in \mathfrak{b} \&$ (Astacus fluviatilis) bat fdeerenfórmig gebils Dete $\mathfrak{B o r d e r f u ́ f e ́ , ~ D e r e n ~ e r f t e s ~}$ Paar fegr grof́f ift, und einell yon tals figen Salbringen beocéten sjinterleib, Defien Eeitenendfoffen aus zroei Etiden beftegen. (Er wird gegen $6^{\prime \prime}$ lang, ift in Leben braun, itm Tode roth, findet fid in unfern füsen ङemáffern und nábrt fid yon thierifden Subitanjen, befonders 2 lab.

Der Ėinfied ften Jufpaare $\mathcal{E}_{\text {d) }}$ eren und einen nidft von EalEigen Salbringen be=

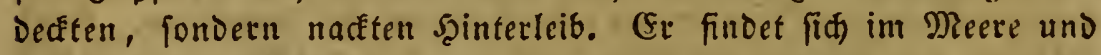

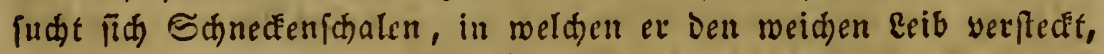

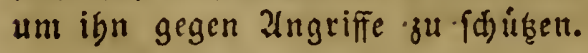

B. Mit Eleinem Scinterteibe, ber Eeine Floffen am (En= be hat, uno im $\mathfrak{E}$ eben gegen bie Bruft nach vorn umgeichla= gen ift. Surzichwanze,

Det gemeine $\mathfrak{T}$ a f

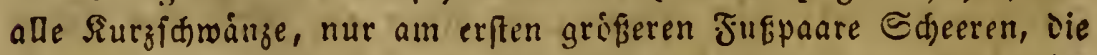

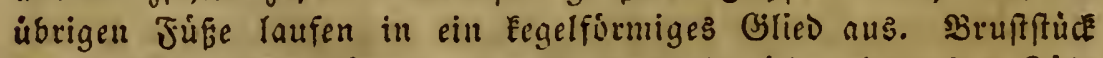
breit, faft halbereisformig, am 20 orderrande ieverfeits mit 9 3ág: nen. B̧emrin in Den Meeren an Deutfden Siúften. 
Siebente Silnffe. Siruftaceen. Poocilopoden. Spgyllopober. 70

\section{Sedbte Familie. Stadelfüß̈er, Poecilopoda.}

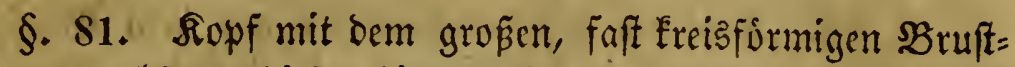
ftut werwach)en, biefes binten tief ausgefchnitten jur $2(u f=$

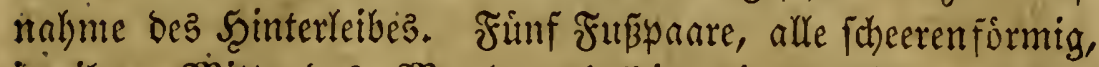
in ilyrer Mitte bas Maul, und bie mit Stacheln beferzten Sciften vertreten bie Stelle ber אiefer. Ritemen unter Flap= pen am Scinterteibe, biejer louft in einen Stachel aus.

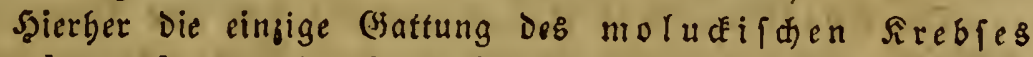
(Limulus moluccanus), ein grofes Thier von 1' Durdmeffer und brauner Jarbe, Das an oftindifden Siuffen gefunden wird.

\section{Siebente Familie. Brattfúf}

§. 82. Sopf bald verwachien, bald frei; Maul mit 2 groß̈en, ftarken Siejern; Dahinter am \$Bruftftuce viele blatt=

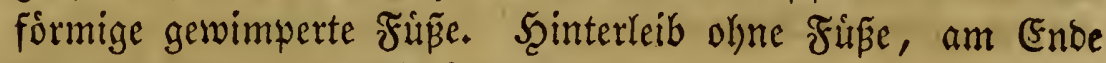
mit Borften oder Schwimmblittern. 3. $\mathfrak{B}$.

Der $\mathfrak{S}$ Iatt $f$ uf (Apus cancriformis), mit wermadjenem fiopf

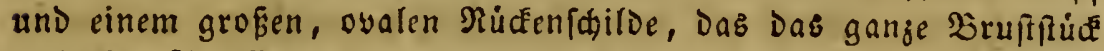

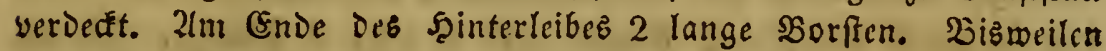

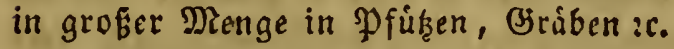

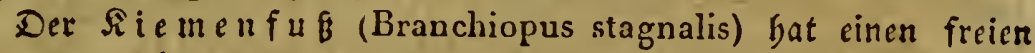

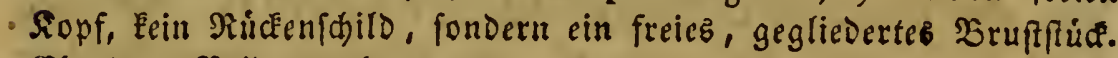
Ebenda. Beide 1" lang.

\section{2(b)te Familie. $\mathfrak{B}$ it $\mathfrak{f}$ delfúper, Lophyropoda.}

§. 83. Siopf mit bem sBruftitiue verwachfen; an ihm 2 ober 4 oft aftige Finhler uno ein eingiges mittleres 2fuge. leib bald beutlich gegliedert, bald von ziwei gropen, flappen= fórmigen Schaten zum aheil ober vollfommen bedect. Beine

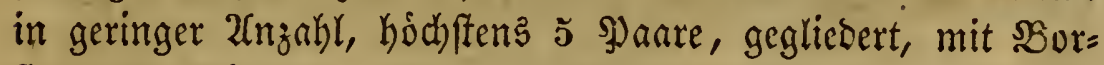
ften uno $\mathfrak{M B i m p e r n}$ bejeşt. 3. $\mathfrak{B}$.

Das E i n a u ge(Cyclops quadricornis) bat einen geftreckten, icut=

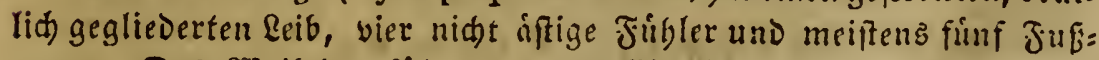
paare. Das Tiseibdjen fübrt 2 grofe cierfacke mit fid berum. In B̧ráben und Đfúk̨en.

Der $\mathfrak{W}$ afferflob (Daphnia pulex); Eeib yon zwci ETappigen ङめalen umidlofien, aut melden nur Der Siopf bervorragt; 2 áftige Júbler, 5 Jubpaare; Farbe feiforroth, Darm grin; fo grof wie

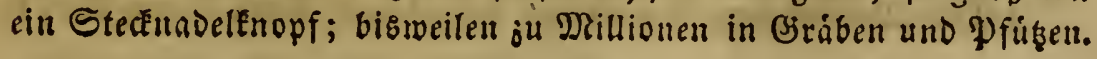




\section{Theunte Familie. Ranfenfǘcr, Cirupedia *).}

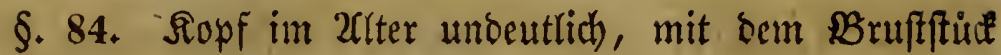
verwad)fen, augenlus, in oer \$ugend mit einfachem 2üge uno 2 Fublert. 2 şaare tofterlofer Siefer, 6 Fußpaare, jeder

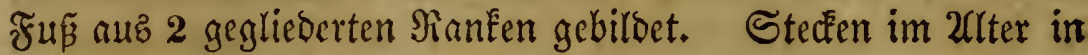
grop̈en Ealfigen aแb melyren Studen zujammengejeften Scha ren, bie mit einem báutigen oft ftielformigen (Siunde feftfisen.

Die (Entenmufdel (Lepas anatifa) bat eine finffdalige Salegúlle, Die von einem langen bautigen Etiel getragen wirb. Thicr mit fünf paar bipfelformigen Siemen uno rangem fdwangs fórnigen 2lngange zroi fhen ben Jüfien. In allen Deeren.

Dic Seepo cée (Balanus tintinnabulum) bat cine aus megreren Etiden jufammengemadjene, Eegelformige, oben offene Sdjale, Des ren Eingang Durd 2 Dedelidalen veridloffer wirb. Die. Sdale fiģt ofne Stiel auf 2ufteridalen, Steincn, 3e. Das Sgler mit 2 grofien gefaltener Siemen.

3ebnte Familie. Schmaroberérebie, Parasita.

§. 85. Slopf mit bem SBruftitud verwach 5 en, an ifm in Der Siegel 2 ungeftielte 2ugen unb 2 ober 4 Fúleler. S) Raul melyr voer weniger idnabelformig, mit borftenformigen Sie= fern, ober eine blofe Seffnung, obne alle Siefer (?). S3orber= beime bafig jum 2fnflammern, bie binteren floficnformig; bochftens 6 Fuppaare, bisweilen gar feine, fonbern blope flei: ichige Rappen an beren Etelle. Die Ihiere leben als Echma: roker auf Fifhen und find von unbebeutender (sirópe. 3. $\mathfrak{B}$.

Caligus piscinus, mit 2glicorigen Jihlern, 2lugen am Solver: ranie eines iebenferts vorn vom 2 ruff fdilde entipringenden fort: fakeze, mit riffelformigem Diaul und 6 Juppaaren, Die zwei er|fen

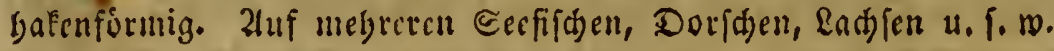

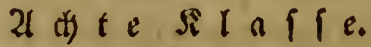

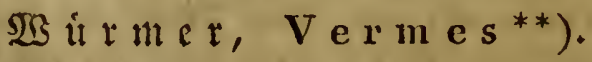

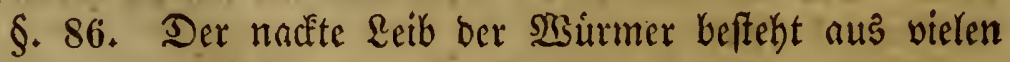

*) Serglcidje meine 2ubanblung: Weitráge zur Saturgefohichte Der ßanfenfüfer. 'Betlin 1834. 4 .

*) Die Seteinigung Der bisget unter Dem Siamen yon' Roflj= 
unter fith gleid)en Ringen, bie oft fehr beutlih), bäuffig aber aud) mur fidsuach, abgeferzt finto. (Sticomaßen finden fich) noch an biejen Siingen, ood) eigentlich bann nur mit Beftimmtheit, wenn zugleid) ber Sopf mit Fukhlern und 2(ugen beutlid)er hervortritt. Eaz fino in biejem Fall Eurze, hócferige, mit $\mathfrak{B o r}=$

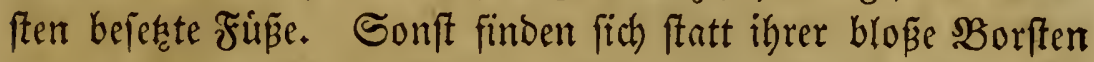
ober grozé, muskulófe (jruben, Saugnappe genannt. Sie liegen bann, wie bie Finse, am Banch, meiftens an \$orbers uno Seinterente; auth liegt wohl bie Munooffnung in einer foldhen Sanggrube. 2uf ber $\$$ berfläche ftethen bei Sielen lange, feine ober bidfere Borften, bie ben Thieren cin haraiges 2(n. fehen geben, Daker man fie, weil fie im Miecre leben, Meer= raupen zu nennen pflegt. Sft wirb bie Raubigkeit ourdh

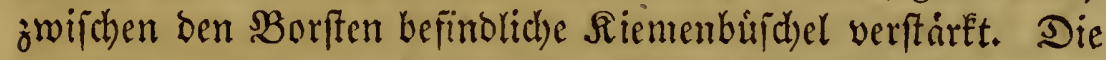

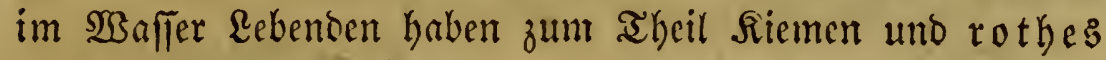
Blut in Deutticken (Sefofípen; bie Uebrigen leben im Inneren

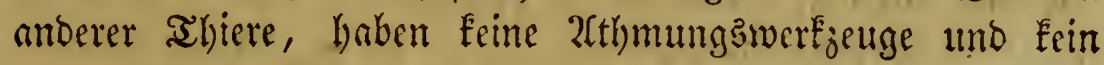
rothes Şht. Diejen ift Dagegen ein gabclfórmig getheilter, mit vielen Nebenáften verfebener Darm, eigen, ber blind endet, alfo keine 2lfteriffnumg hat; bie, erfferen haben einen einfa= dhen, graben ober mit weiten $\mathfrak{I} a\{d$ en veriebenen Darm nit 2ffteroffinung am Seinterleibe.

Man fann bieje Slaffe folgendermaßjen eintheilen:

wirmern ober Unnelioen, Gtruberwúrmern uno (5) ingemeio w it mern als getrennte Silafien aufgeftellen Gruppen in eine cingige filaffe, bedarf yor bem fachundigen Publieum eines nalperen bemeifes, Der nidt bierber qebort; es

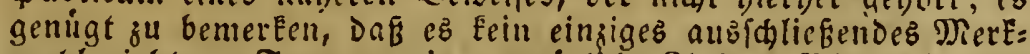
mahl giebt zur Trennung in 2 naturlide Slaffen, Ringel = uno

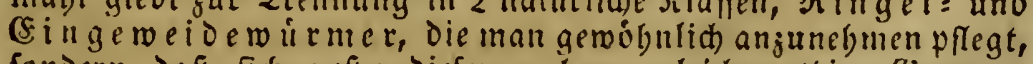
fondern Daf fid aufer biefen mehtere gleidwettbige Sruppen finden, zu weldien aus beiben Ilaffen fid entipredende und er: gálzende Silteder gebsen. Diefe Gruppen babe id vorláfig als jamilien bezeidnet; Die umftandidye Bemeisfúbrung bebalte id) mir fur Die ZuEunft bei befferer Sielegenheit yor. Jie $\mathfrak{R} a$ dertbiere, welde id friber Den Sumern verwand glaubte,

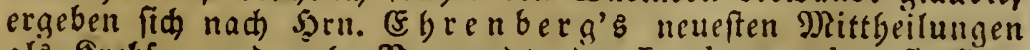
alz Ireble, und nabe Serwande Der Lophyropoda; fie find, als unbeocutende Hebergangsgliever, jetgt ganj auggelaljen. Bergleide mein Saandbud Der Siaturgefdidhte. Berlin 1835.8.

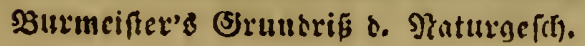


A. Mit brebrundem Reibe, woran SBor= ften voer wahre Fuffe. Darm grinde,

Erfte Sionung. Arthrodes. mit 2 fter.

a. Mit rotbem SInt und Siemen. 1. Fan. Piotbrutuner.

b. Sieine Siemen, Dlut totb, fteife $\mathfrak{B o r}=$

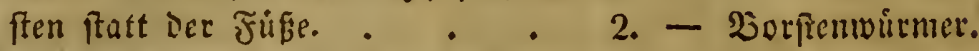

c. Dgne Fiemen und aufiere Drgame. Slut meif̂. Leib rumb. • . 3. - JaDenwirmer.

d. Seine Siemen, Blut meiß, Der Reib flad, zun Theil mit \$Bimpern verfeben. 4. - Gtrubelwirner.

3. Mit flachem, fhroch ober nicht ge= 3meite Dronung. gliedcrtem $\mathbb{E}$ eibe, woran Saugguben. Trematodes.

a. Leib lánglid, mit 2lfteriffnung. Zlut roth. . . . . . 5. J5am. Blutigelwitmer.

b. \&eib veranderlia, Darm gabelig ofne 2ffer. Eine Sauggrube, worin Der Mund. . • . . . 6. - Plattwirmer.

c. Leib elliptifh); Darm gabelig, ogne $2 l f=$ ter. Mifrere Sauggruben. . 7. - Saugmirmer.

C. Mlit runoem ober flact)em, Deutlich gegliebertem Reibe; Sopf mit Gaug= gruben oocr Satern, ober beiben zu= gleich. Sein 2ffter.

Dritte Sromung. Helminthes.

a. Sispf cin eingiebbarer, bakiger ఇiffel, \&eib rund. . . . . . 8. Jam, Stadieleopfro. b. Siopf celiu; \&eib fach, bandformig. 9. - Santwurmer.

c. Siopf eckig; Seib flad, chlot in eine Diafe.

10. - Slajenmirmer.

Erfte Frantilie. Rothrourmer, Annulati.

§. S7. Sie baben cinen runden $\mathfrak{\Omega}$ cib mit beutlich abge= pesten, gleichen Sliebern, Deren jebes. an ber $\mathfrak{B}$ auch feite mit fubartigen Spoctern, die Borften tragen, werfeben ift. Der vor= Derfte Sint bat bis.weilen cinen abweichenben $B$ au, trigt bann geglieberte, Fibler unb 2 - 4 einfache alugen. Siemen âuer= lich buifhelformig, theils auf bem ganjen \&eibe, theils am

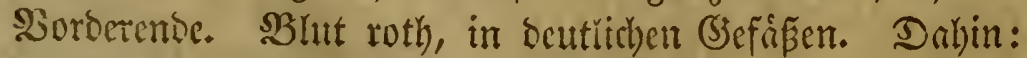

Nercis pelagica, mit Deutlichem Sopf, wotan mefr als 2 Jifis: Ier uno cin 2) haul mit 2 gezónnten Siefern; 4 2urgen; Drei Eleimc 


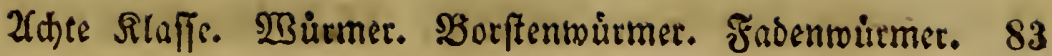

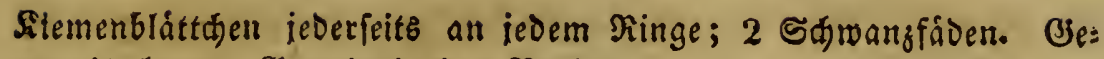
gen $4^{\prime \prime}$ lang. Bemein in Der Plordfee.

Serpula vermicularis (WBurmrobre); Rein Deutlidfer Sopf,

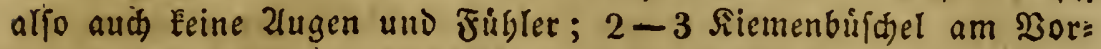
Derende Des Sörpers; Fuffbúcker mit Eurzen Zorften, längere auf

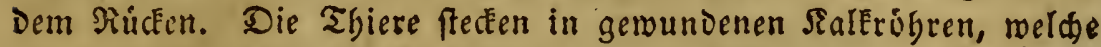
won Der Deerfádde thres Sivrpers abgefondert werden, unt die fie

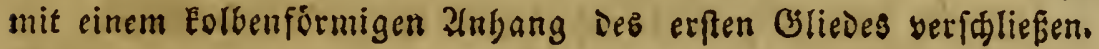
givrofee.

\section{Sweite Familie. SBorfitenwirmer, Chaetopodes.}

§. S8. Shr Leib ift brehruno, beutlich geringelt, mit. fiteifen Borften an ber. Banthleite, theils an allen, theils an

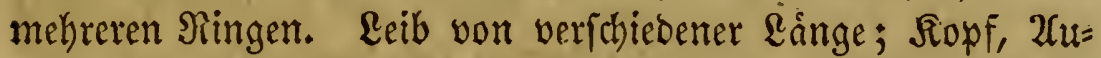
gen, Fithler nicht bet athen bemerEbar; bisweilen Fàben neben dem Numbe. Eeben im Schlamm bes ufers ober in feuch)= ter Froe z. $\$$.

Der $\mathfrak{x}$ gennurm (Lumbricus terrestris) Gat Eeinen Deutlis

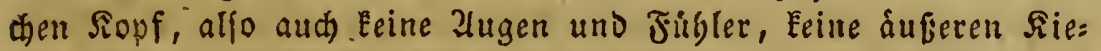

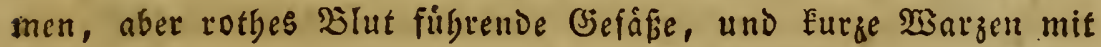
fteifen Borften in 4 Doppelreiben am Baud uno an Der Seite. 2luf ein Drittel Des \&eibez ein Didererer Ring (Sattel: (jürtel). Rebt

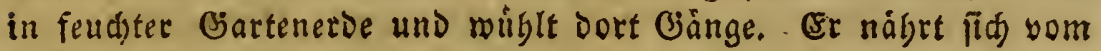
Erbfaft (humus).

Die Ta a den baben einen jatten, feinen, Durwhidtigen, mit einzelnen ober mefreren Boorften iederfeits an jeoem Ninge befeştêt Qeib; bei manden finden fid Zlugen; bei Eeiner áufere Fitemen. Nais proboscidea gat einen langen Jüblfaben (Rilffel) ant Der Sberlippe, 2 ?lugen uno 2 borften auf Dem Rürfen, 4 am bauds an jeder Eeite jedes Ringez. Pfanjt frd and Durd) Theilung fort. Sn ftehenden Gemáffern. Die meiften? ভdiamm.

\section{Dritte Frnutie. Fabenwà}

§. 89. Sic haben einen langen, brehrumben, meift bint= nen Reib, or)ne alle áüeren Sorgune, als Fupwarzen, Borften u. Dergl. Die Singe des eibes fino Furz und nur (t)wach)

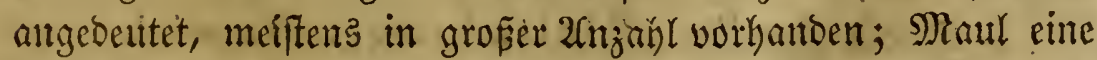

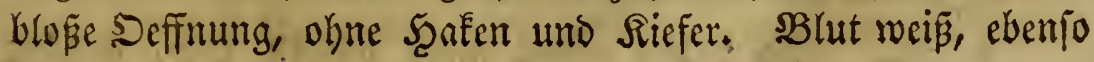


ber Sirper. Sie leben meiftens in Snneren anderev Shiere, bejonders im Darméanal. 3. $\mathfrak{B}$.

Der Spulru $\mathfrak{r} m$ (Ascaris lumbricoides), ein langer, feder=

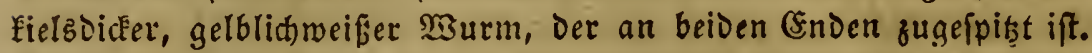
Maul yon Drei Sinothen umgeben. Lebt im Darm Des Renidhen, befonders Der finder.

Sierte Familie. Strubelwúrmer, Turbellarii.

§. 90. Der $\mathfrak{L}_{\mathrm{e} i b}$ ift langlich, flach, banbformig, wenig geringelt, bisweilen geich)wanzt, nuf Der Sberfliche fein ge= wimpert, nicht zum Schwimmen gejejict'. Ueber ber Mumbs offnung nicht jelten mehrere 2ugenpunfte. Sarm weit, nicht gemunben, mit 2ffteróffnung. SBlutgefápe bemerťbar, Eeine 2(th)mungsorgane. Einige pflanzen fich burd) atheilung fort.

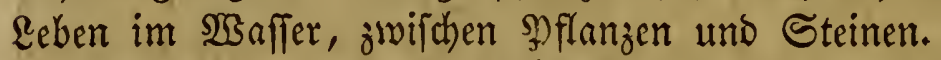

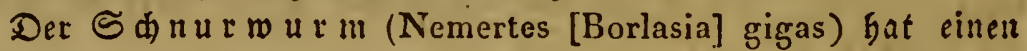
fladtrunden Reib, eine bordere Mundöfinnung, Gintere 2fferófinung, und eine grofe faugnapfförmige Befdledtsóffnung am Borterleibe. Mird bis 6' lang, lebt grwifden Eteinen an Den Siliften yon Enge Yand, liegt zufamnengeEnauelt. Farbe braun, itts Brtunlidje, mit 5 belleren Längştreifen.

Finfte Familie. $B \mathfrak{l}$ li tigelwürmer, Hirudinei.

§. 91. Shr $\mathfrak{L} e$ ib ift ebenfalls nadt, aber nicht lang, meiftens nur 3-4", babei flach, breit, nach beiben (Enden zugeipist uno fojwach geringelt. Mundoffnung in einer Saug= grube, eine zweite am Schwanzende unter bem 2 fter; Darm

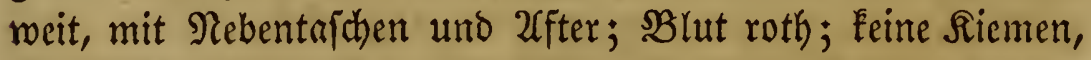
bie Sảfe an ber Bauchleite find Schleimorgame. Reben im

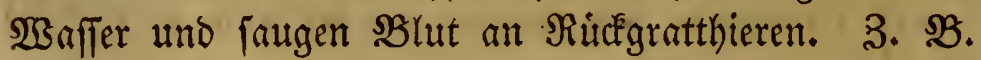

Der medicinifda $\mathfrak{b l u t i g e l ~ ( H i r u d o ~ m e d i c i n a l i s ) , ~ y o r . ~}$ Derer Saugnapf nid)t abgeferęt, blos Der erwoiterte Miuldorand; im Munde Drei lanzettformige Sirfer mit gejägnter Sdneide. 10 2lus gen. Farbe oben braungrin, mit 2lugenflecken in 6 Reiben, unten

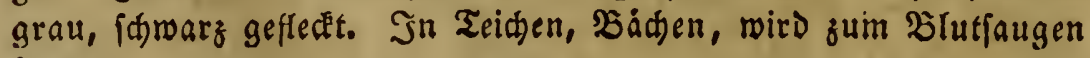
benukgt.

Sedyfte Familie. Shlattwairmer, Planariei.

§. 92. Sie haben einen melyr flachen \&eib, weldher 


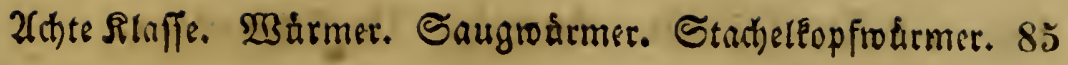

Feine beutliche Singelung mehr jeigt, aber, bejonders bei ben

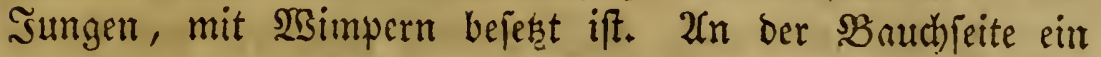
Snugnapf morin ber Mund; am Sorberende 2ugen, bie bis= weilen fehlen. B̉lut weí̈. Darm gnbelförmig, verżmeigt, ohne 2rfter. Sie leben im $\mathfrak{B a f f e r}$ 3. 3.

Der mirtweife plattwurm (Planaria lactea), Raul

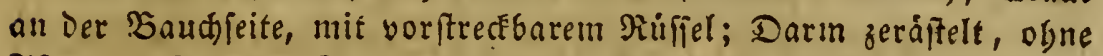

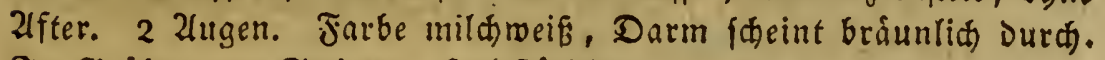
Jn B̉ráben, an Steinen, Şotgltideden u. f. w.

2Indere 2trten uno Battungen leben im Deere, zmifhen Zan: gen und Sorallen.

Siebente Familie. Saugwurmer, Trematodes.

§. 93. Sie haben ganz die Gieftalt und Bildung ber Sorigen, aber ihr leeib ift glatt, ohne W3imper. Saugnaipfe

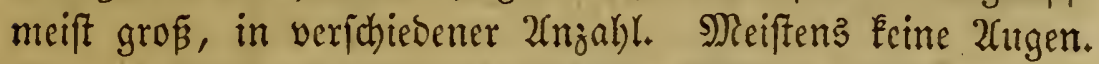
Sie reben idhmarofent an anderen ahteren, bejonders im Darm' z. $\mathfrak{B}$.

Der \&eberegel oder Die Egelfánede (Distomum hepaticum), mit grofer Eauggrube am \$auth, einer Eleineren, worin Der Miund, uno cinem Dritten Eleineren (frúbd)en Dazmifden, wo: rin ber Gierfito mindet. Darm ftark zeriffert. Form und Brobfe eines Eleinen Diofenblatts; Jarbe gelbliff. Tn Den Baalengången Der Edjaafe und Irenifhen; bizmeilen fegr gemein. 2lndere Bat: tungen finden fid fogar im Zluge ber Jifde, mande and a du ferlid an Dell నiemen und auf Der Şaut Der Fifde.

2(ch)te Familie. Stachelfopfrourmer, Acanthocephali.

§. 94. S Sin biejer Familie findet fich ein ziemlich langer, immer brefrunder $\mathfrak{L} e i b$ wieber, welcher nach vorn gemeiniglich

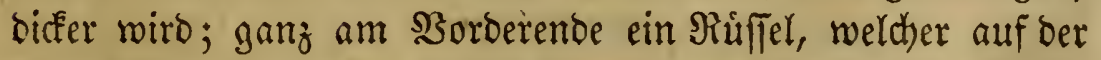

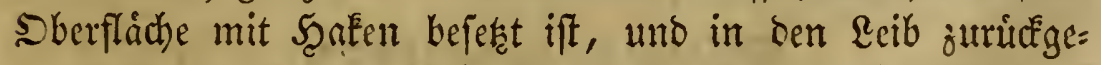
jogen werben Eann. 2(fter fehlt. Şierher bie Ssattung:

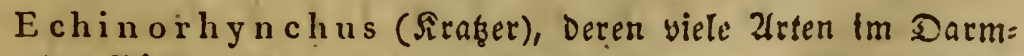

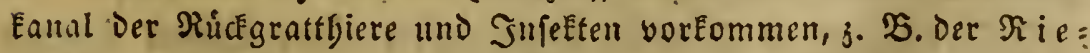
fenkra ger (Ech. gigas) beim Sdwein. (Er wird 1' lang, ift fes

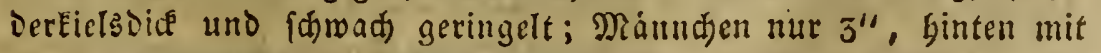
einetn glockenartigen 2uthange. 


\section{Erfter 2t6iannitt. 30otogis. II. Bsteberthiere.}

\section{Neunte Familie. Banowũrmer, Cestodes.}

§. 95. $\mathfrak{L}_{e} \mathrm{ib}$ lang, flach, beutlich gegliebert, von hinten nach) vorn verichmálert, am fpiten \$orberende ein ediger, mit 2 voer 4 Sauggruben uno oft cinem Scafentranz um ben

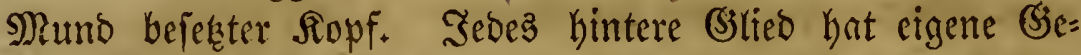

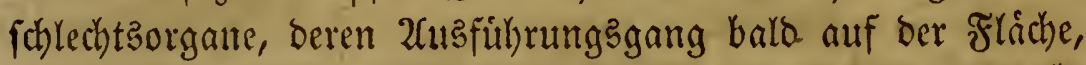
barb am Ranbe liegt. Sie Yeben im Darm und in ber $B$ auct)=

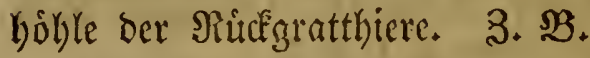

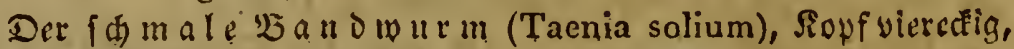
mit 4 Sanggruben uno ŞafenEranj. B̈lieder quadratift. Bes fdjledtsoffnung abivedfferno am Miande. Sm Darm Des Dienfden, befonders in Deutffland, Jrankereid, England, Sdmeeden.

Dor breite $\mathfrak{B} a \mathfrak{n} D \mathfrak{w} u \mathfrak{r m}$ (Bothriocephalus latus) bat cis unen Iángliden Siopf mit 2 Sauggruben; breite, quer red)teçige

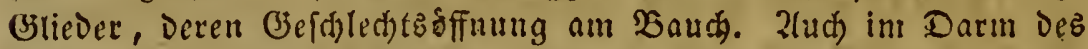

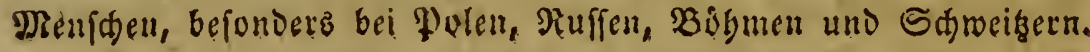

3ehnte Familie. Blafenwurmer, Cystici.

§. 96. Sie haben cinen furzen, gegliederten, flacten, \&eib, Der binten in eine Blaje aublault. Sopf Der Sorigen. Wie bieje olyne 2ffter, mit gabeligem Darm. $\mathfrak{L}_{\text {eben }}$ nuch im Inneren ber ILbiere, aber nie im Darm.' 3. B.

Die Jinne (Cysticercus cellulosae), Die blafe miro fo grof wie ein Sarrotern voer eine (Erbfe, und gebt allmálig in einen Reib

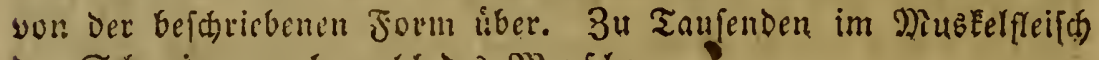
Der Sdimeine, aud wobl Des Mien[dien.

Der Drebiarm (Coenurus cerebralis), 2ln einer.einzigen

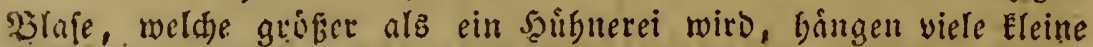
Eeiber yon ber befforiebenent Beffalt, weldhe fid rủckwárts in bie

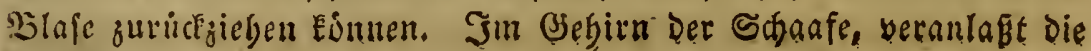
Decherankbeit.

Dritte Scauptgruppe.

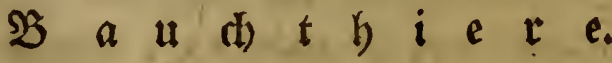

§. 97. Das ficherfte Rennjeichen biefer britten Scaupt= grupye des aghierreiches liefert ebenfarts bie alfgemeine Form

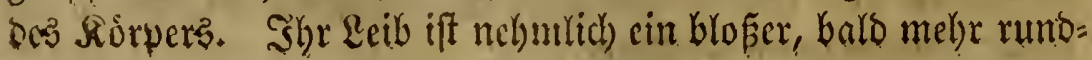


licher, bart flacher, fdueibenformiger, bald auth limglidyer, aber ftets ungegliederter Sacf, in welchem die oft felbr vollewumen

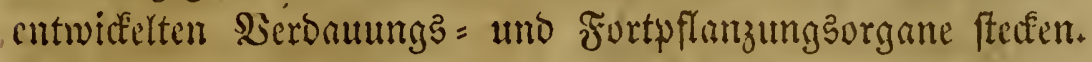
Un biejem \&eibe finben fich wohl cinige ober viele, hiulfig outcl) bie Babl vier oder finf ohne Reft theitbare, reget= minsige 2lusftrablungen, welche in einigen Forfen fogar aus bintercinander liegenden, won Scaut befleibeten, gleichen Sinv= d)englicbern befteben, aber nie erfennt man an biejen Strah=

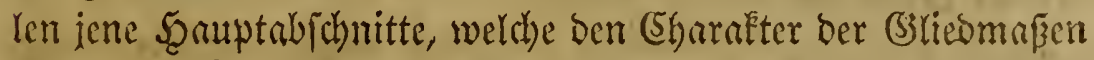
bilden, wicber; immer nebmen fie vum 2fnfange gegen bas Enve bin an Uumfang ab, unb befteben oub gar Peinen voer

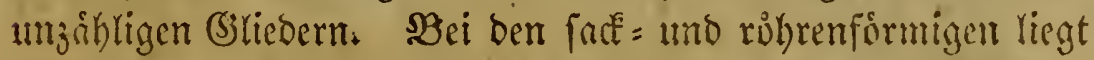
ber Matmo vorn, bei ben fackibenförmigen in oer Mitte an ber Unterfeite; um ihn fteken meiftens cinjichbare Füblfión, vier lange, hoble, nicht einjichbare Fangatme. Bei mantb) bededen ben 2 eib cine, zwei voer mebrere Salfichalen; bei anteren ift er nedet, aber mit vielem Schleim tiverzugen; bet noch anderen fest fich bie Sirlemafle im Snneren $a b$.

Unter ben inneren Srganen felyt ber Darm wohl nic; oft ift er ein gewumbener Sanal mit Schlund, Jlangen, Dinn=

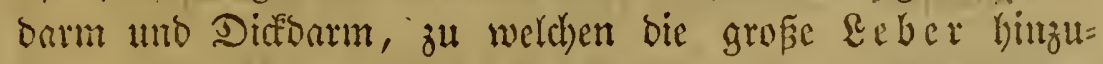
fommt; bet anberen iff er ein bloperer cinfacher geithlingelter

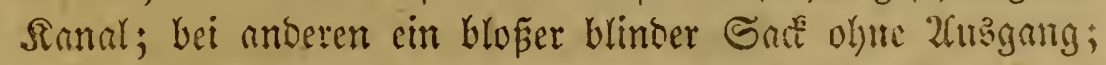
bei wisber anderen hingdn vicle rumblicke sldagen an bum binten offenen Darménal. Die Fortpflanjumgsorgane be=

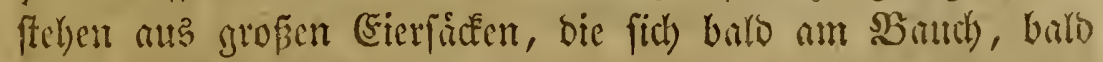
in ber Rikb Des Mundes offuen.

Son Gimnesorganen finden fich nod) 2(ugen, ia fetbit

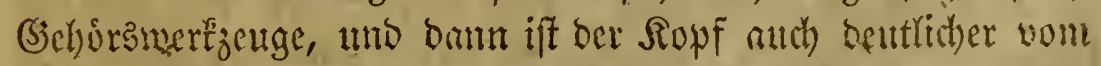
idbrigen Leibe getrennt, allen anderen fel)len Sopf und Sin: neşwerf zcuge vollépmmen.

Die allermeiften biefer aghere Yeben im SBaffer, bejenders im Meere, uno freffen bann thierifche Narhumgamittel, cinige, Die auf bem $\mathbb{a} a n d e$, aber nur an feudsten, fhattigen Stellen worfoumten, freffen auch sBreitter.

פ) 
S8 Erfter 2rbjonitt. Zoologie. III. Bauchtbirere.

a) Meichthiere. Shr weicher, von 1, 2 ober mehr $\mathfrak{R a l e}=$ ichaten umgebener $\mathfrak{L}_{\text {eib }}$ hat einen weid)en, vielen Ed)leim abjondernoen Scautlappen (Mantel), welcher ben gan= jen Riviper unthitllt. Maul vorn.

b) Strahlthiere. Şhr flacher ober Eugeliger, felten $(y=$ linorifcher $E_{e i b}$ ift von kalfigen ober Yebernatigen Echa= len umgeben uno lianft in 5 oder 10 Scauptftrablen ants, in beren SRitte bas Maul.

c) Souallen. Shbr meiffens flacher, feltener blaifger, gat=

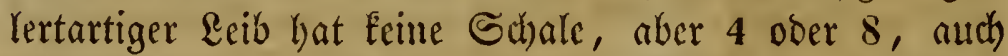
vicle Fangarme um ben mittleren Mund, ber jebod) ei= nigen feflt; ftatt feimer bann viele Saugróhren.

d) Polnpén. Elib gallertartig, runolich, mit voroerer, von Fưhlfáben umgebener Munooffinung. Siele ahbierchen ' ourd) gemeinfame Scaut zu einem affigen ober ftrahli= gen Stumm, der innen ober auß̌en SalEmafle abję̧t, verwachien.

e) Snfufionsthiere. Leib flein, gallertartig, frei ober ourch Stiele zu, einem gemeinichaftlichen Stumm ver= wachfen. Inmenbig viele rundliche Magenblajen.

\section{$\mathfrak{F} \mathfrak{e}$ u $\mathfrak{n}$ te $\mathfrak{R} \mathfrak{l}$ a f $\mathfrak{f}$ e.}

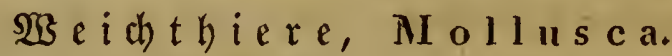

§. 98. Scierker bie einjigen $B$ audhthicre mit beutlichem Sopf und Simnesorganen, weld)er jebod) nicht einmal allen

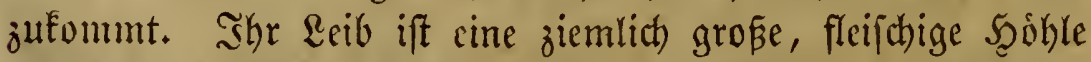
mit vorberer Mundiffnung. Son ben Seiten bes Ritctens entfpringt ber meiche, häutige Mantel, welcher theils ben ganjen Reib, theils nur bie (Fingeweibe eingullt. (5r jonocrt bie karfigen Schalen $a b$, von welchen bie meiften SSSeichthiere umgeben werben. Unter bem Mantel liegen jugleich die '2(t)= mungsorgame, bei ben meiften faltige voer bijchelformige Sitemen, bei anderen eine großße faltige \&unge. Wake baben einen wahren Darm, mit 2ffteroffinung umb ciner großenen

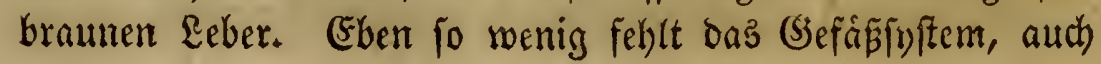


ift immer ein Scer ba, von welchem bie Bewegung bes blint=

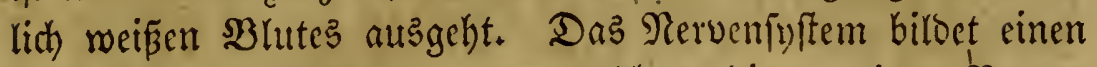
Ring um ben Sdhlund, von weld)ent bie paarigen Serven ful ben ibbrigen S7rganen hingehen. Bei ben meiften bildet bie untere Sante bes Baudhes eine fleijchige Schwiele, auf welcher fie fortfriecthen, und bie baher ben Siamen $\mathfrak{F} u \tilde{\beta}$ erhal= ten Kat. Die Ealkige Schare ift theils eime gemumbene Siohre, theils beftelt fie aus zwei flappen, felten aus melyeren glei= dhen ooer ungleichen Etitfen; manchen fehlt fie.

Man theilt bie SBeichthiere folgendermaß̄en cin:

A. Mit Deutlichem Sopp, woran Frihlfiben unt 2Cugen.

Sopf mit grofien, langen Jangarmen;

Mantel facfformig, vorn ofien. . 1. Fam. Siopffíper.

Siopf mit 2 ooer 4 eingielgbaren Jüblern;

Nantel meift grof, Die CEingemeide um:

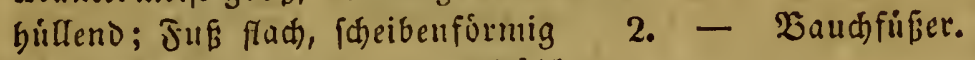

Siopf mit Eleinen, cingiegbaren Juiblern;

Mantel Elcin; JuÉ foffienfórmig. 3. - Rielfúf̧cr.

Sopf Elein, neben ibm 2 flingelartige 2lus:

breitungen des Jiantels. •. 4. - Jloffenfuffer.

B. Fein Deutlicher Sopf.

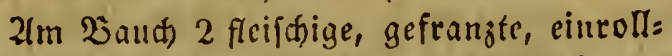

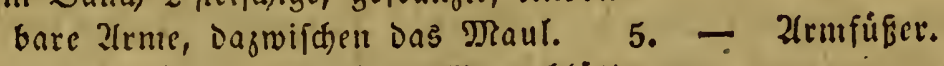

Saud) Eielfórmig, Daneben 2 Paar blätts

rige, inwendig gefaltete fiiemen. Ran=

tel meit. 2 ङfalen . . 6. - Mufdeln.

Baaud gan o von Dem nutr born und binter

offenen Mantel unffoloffen, Siiement in

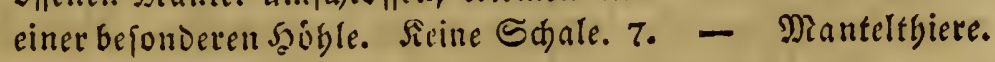

Erfte Familie. Sopffíf $\mathfrak{B} \mathfrak{C}$, Cephalopoda.

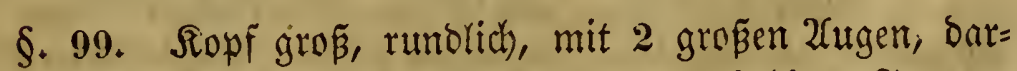
ibber bie Nundoffnumg, von ben langen, fleifhigen Fangar= men umgeben. Sm Mraul 2 Siẹer uno eine gezábnte Sun= ge. Leib wie ein Sač, wegen bes groß́en, vorn offenen Mantels, in welchent, außerbalb bes నorrpers, bie Riemen

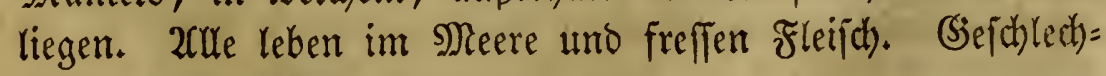
ter getrennt. Seierher :

Der Dintenfifí) (Sepia officinalis), mit 10 Z(emen, wovon 2 
långer; Ecib länglich, ieverfeitz mit ciner flügelformigen Xusferi:

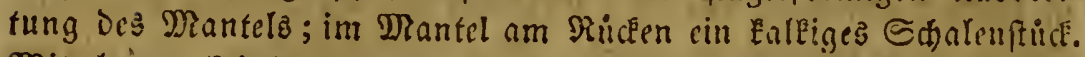
Dittelmeer, Eried) auf Dem Greunde mit Dem Daul nad unten, bat elne mit brauner Jarbe gefüllte BInfe, aus welcher die braune Jarbe Sepia bercifet mird.' Diefen Saft likgt er bei Bifabr ins $23 a f f e r$, trübt Daffelbe und entgebt Dadurd feinen Teinden.

Der Papierna utilus (Argonauto argo) bat 8 Zrme, moson 2 flofienartig erweitert fino. Das Thier ftedt in cinet meifen, gee rippten, Labnfirmigen Schale, mit melther es auf Der Dberfläde Des Reeres feegelt. Dittelmeer.

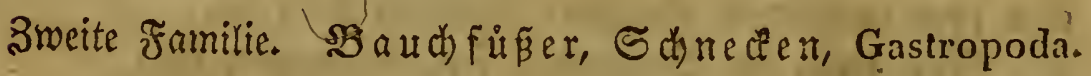

§. 100. Ieib langgeftredt, mit beutichem Sopf, woran 2 ober 4 fithler, bie finteren mit 2 uggen am (sirunbe oder

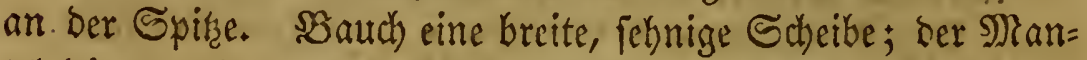
tel binn, die Eingeweibe umbillend, meiftens mit biefen in ber robrigen, fpiralformig gemundenen Sdyale verftedt. Sie athmen meiftens burch Siemen, einige auch Durch Intngen; biefe fint alle 3mitter, die Ulebrigen bald getrennten (Sic=

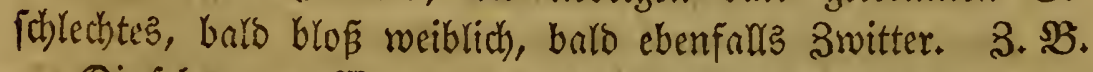
- Die f ovalem, flachem, fdildoformigem Mantel auf Dem Rưuen, unier wels dem fith Der Reib verbirgt. Eunge ein faltiger Sad im Mantel, am redten Rande Defielben Die Deffrumg. 2h feucten Stellen im Marbe, rotbbraun oder fdroarz; frift ¿egetabilien.

Die grofe B̈artenfdued e (Helix pomatia), ebenfall mit Qungen, und. iener im Bau ćbnlid, aber ber Mantel fteckt mit Den Eingerweiden in einer gewundenen, glatten Echale, Deren letste win=

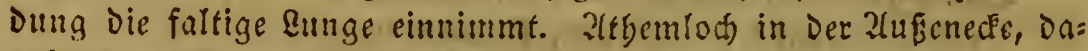
neben Der 2lfter. Erreidbt über $1^{\prime \prime}$ in Durdmeffer. Jjarbe braun. grau. Sn feudten Gebufdan; wird gegefien.

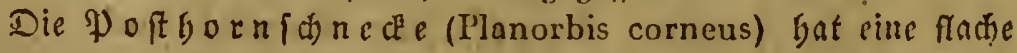
Sdale, Deren Windungen alle in ciner Ebcne liegen. Bas. Thier

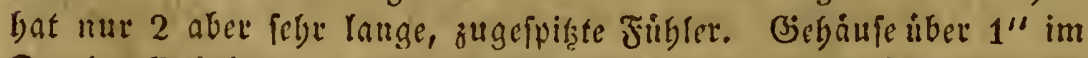
Durdameffer, braunfdmarg, Thier idjwarggrau. Sn füfen Oberáf: fern, Eommen an Die Dberfidg, um frifde Ruft in Die Eungen auf: junefmen.

Die mit Siemen verfekenet Schneden tragen bicfelben an felyr verichiebenen Stellen; bet ben meiften litgen fie im Raufen bes shieres in einer befunderen Soble, uno baben,

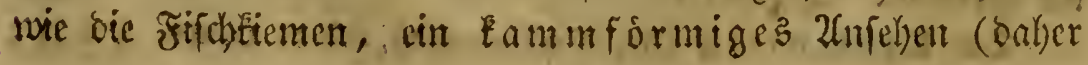


Nerunte Sinflfe. Moftusfen. Sceteropoden. Noteropoden. 91

Rammfiemer); fie fino getrennten (jefblechtes. SBei an= beren liegen fie an ber recten Seite und werben vom Man= tel bededt (Dachlemer); wieber andere baben auf bem

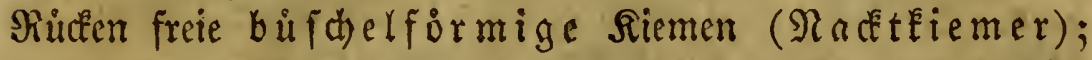
noch altbere baben fie an einer oder beiben Seiten bes $\mathfrak{K u r}=$ pers in einer tiefen Falte zwifthen Mantel und $\mathfrak{L e i b}_{\text {(Sei }}$ teneiemer), uno fino meiftens blós meiblict. Die zathrei=

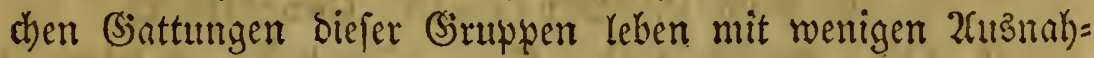
men im Meere, bejonders der aropengegenden* ${ }^{*}$.

\section{Dritte Fanilie, Nielfiufer, Heteropoda.}

§. 101. Sopf beutlich, mit 2ügen und Fiflern, Mraul

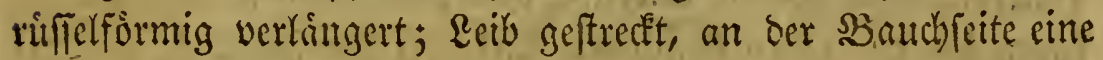
zulammengebridute flolie, bie am Scinterranbe einen Saug= napf hat. Schale Elein, mitzenförmig, zart, nimmt nur bie Leber uno Sienten auf. Schwimmen int Meere, Den Banth nach oben, wo bie zrloffe als Seegel bient. 3. $\mathfrak{B}$.

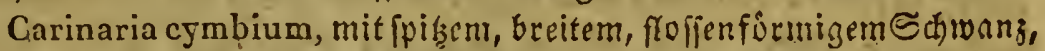
flcincr quergereifter Swale, rilffelförmigem, ausfifteckbarem Naule, gwei Yangen, vorderen Frithlern und ETeinen Ginteren mit Zlugen. Şarbe blisulid. Isittelmeer.

Sicierte Fantilie. Froffenfü äer, Pteropoda.

§. 102. Sowf oft unbeutlich), neben ihm zwei flitgelför $=$

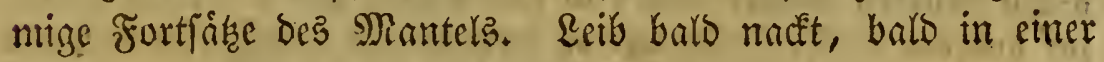
Sibale. \&eben in ber hohen See. 3. $\mathfrak{B}$.

Das $\mathfrak{B}$ a I Ififf a a (Clio borealis), mit Dentidem fiopf,

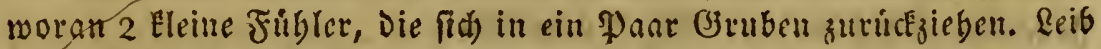
lánglid, flady, you Mantel enge eingehúlth, ofne Sdale; $1^{m}$ lang.

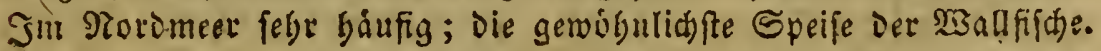

Finfte Familie. $2 \mathfrak{r} \mathfrak{m} f \mathfrak{u} \beta \mathcal{B} r$, Brachiopoda.

§. 103. Siein Sopf, ber Reib fteckt in einer jweiflappi= gen Schale, welche won cinem fleifhigen Fortfats bes athiere?

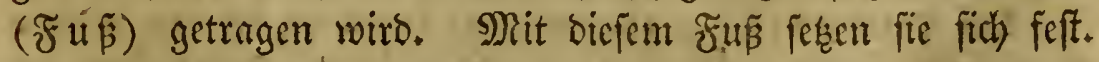

*) Die unendidae Mannigfaltigkeit in Den torten Sdalen uno $\mathfrak{G e}$ :

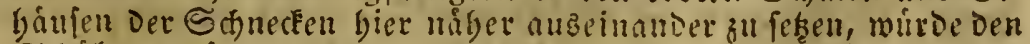
Schuler ermiben, befonverz ba cr in Den Taturgegenftánden feiner Hugebung umfouf nah gieprifentanten fudt; folde Erorterun: gen miffen einem fpejielleren Sortrage yorbchalten bleiben. 
Mund unterharb, neben ithm zmei fleifinige, gefranzte 2 rme,

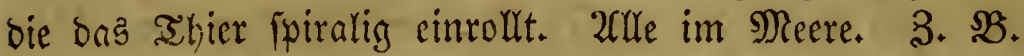

Ligula anatina. Édalen länglid, etwas keilförmig, Dinnt, gleid

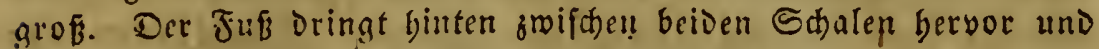
iit fegr lang. Edaaie Gornfarben. Snoifder Drean.

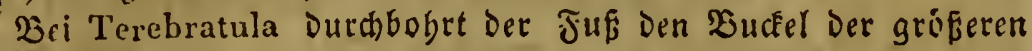
Edjate. Eerenia.

\section{Sechfte Familie. $\mathfrak{M}$ u ibeln, Conchifera.}

§. 104. (5Genfalls ohne אopf. Der Leib vom háutigen Mantel eingehullt, beffen $\mathfrak{L} a p p e n$ an ber $B a n c h j e i t e, b a l d ~ g e=$ trennt, barb verwachjen find; barunter neben bem Reufen bie blattformigen, bohlen, inwendig gefalteten אiemen. Die Bauch = Fante lauft in cinen fleifchigen Riel ober Fortfah aub, auf wel=

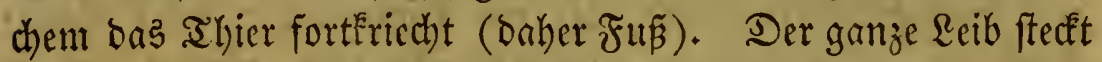
in zwei meiftens gleichen, rumben, nach aulsen gerwilbten Scha=

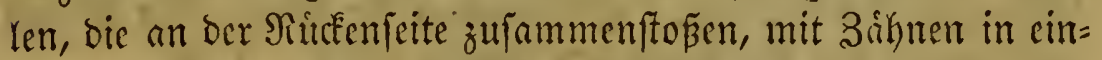

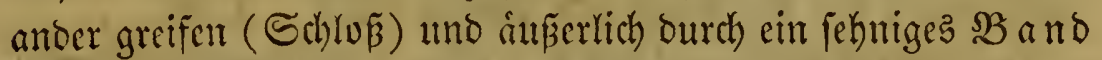
verbunden fint. Mit biejen Schalen fteht bas a ahier burch einen ober jwei Muakeln in Serbindung. Die athiere leben nur im 25 alfer, meiftens im Mecre. Sie bilden, nichft ben

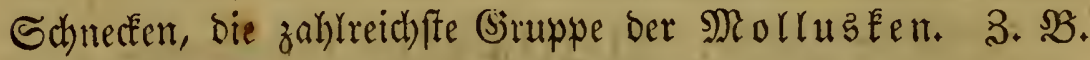

Die 2 lu fer $\mathfrak{r}$ (Ostrea edulis). Sdaien frad, gleid, Durd) e inen bidéen Muzkel, weldher mitten in Der Schale yon Der einen Şälfte fur anoeren binúberläuft, und an jeoer Edfale einen tiefert Einorud madt, verbunien. Mantel ganz offen, am Maande gefranzt. Sallof

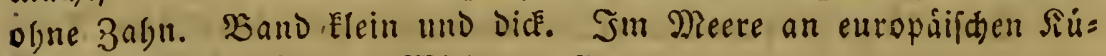
ften, anf Sandbänfen. MBiro gegefien.

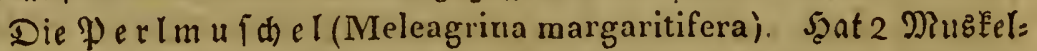

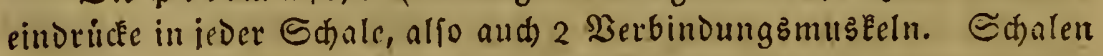

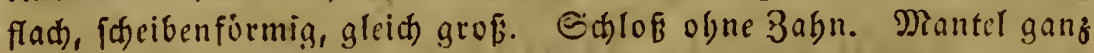

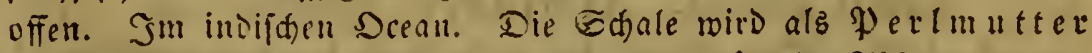
gu Siunffaden bereitrt; Fleine, runde, Eugelformige 26 fonderungen Des Mantele fino die fo gefdrásten ádhten Đerlen.

- Die $\mathfrak{T}$ eidmufdern (Anodontae) finton fïb bei unz in fífen Bemáfiern, baben 2 Jiusfein, gleidie Sdhalen, Keinen $3 a \mathfrak{h n}$ am Eđlof, uno einen úberall offensn $\mathfrak{R}_{\text {Rantel. }}$ Nian Eennt mebrere Zren, Die fid nath-Der Brofife nno form Der Dinnen, inmendig

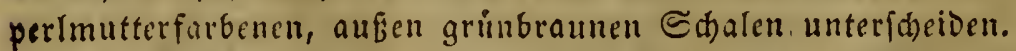

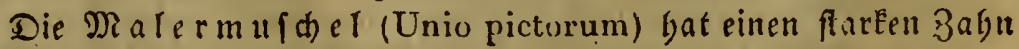

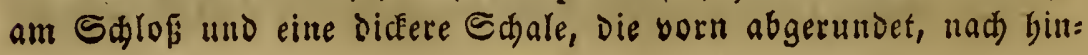


ten zugefpikt ift. Sie findet fich ebenda, ift aber Eleiner, befonbers fămáler.

Siebente Familie. Mantelthiere, Tunicata.

§. 105. Sie haben 2 ergnlichleit mit sen $\mathfrak{B o r i g e n , ~ a b e r ~}$ bie ভahale fehlt; bagegen bildet ber hautige oder Finorpelige

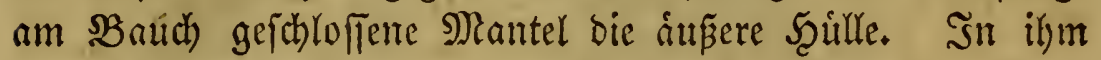
nur 2 Seffnumgen, eine bem Rrule, bie andere ber 2lfter= offnumg bes Siorper's gegenuber; beibe bisweilen bicht neben cinanber. Ritenten in einer eigenen Şoble. Leben mur in Meere; manche verbrciten ein fchimmernbes sicht.

Salpa. Siemenbigle vorn uno binten anf, in ige Die Darm: mindoungen, uno zwar neben Der binteren Der Mutno, úber der yorDeren Der 2ffter. Die siieme als Diameter Durd Die Szible ge: 3ogen. Die 2lrten find frei bemeglid, Die Sndividuen nidit vermads fen, uno fuchen daz yobe Meer.

P y ro s o ma. Wie Salpa, aber Die $\mathfrak{B}$ and Der Siemenfífle ift zuglcid Sieme. Die Sndividuen yerwadfen gu einer Thiergruppe, welde beweglidl ift.

A scidiae. Siemenbjgle mit einer Münoung, aus igrem Grunde entfpringt Der Manno; Der affer múnvet frei nad aufín. Cinige find einzeln, andere verwadfen. 2(de unberweglid angeheftet.

\section{3 e b n te I t a f fe. \\ Strablthiere, $R$ adiata.}

§. 106. Der flugelige, ftrahlenformige ober cylinorifche Leib wirb von einer aus mebreren Salfftiffen zujammenge= fekten, ober lederartigen Schale eingehúllt. Der Muno befin= bet fich an ber unteren Fläche, ober bem vorberen Fnoe, und ift von Ientafeln umgeben. Sm Snneren bes fiorpers fin= bet fich) entweder ein langer, gewundener Darm, ber fich ftet? in einen 2ffter minbet, ober ein bloper Magenfact, ohne 2Cfter.

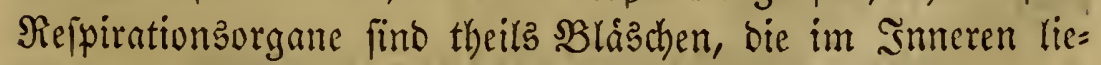
gen, und bas $23 n$ ffer vermittelft der 2(fteroffnung in fich auf= nehmen; oder fie fehlen, und das $\mathfrak{3 a} a$ fler umgiebt bie intte= ren Srgane unmittelbar. Siele beutliche Sefápe; weisez

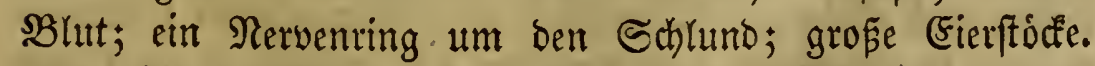
alke leben im Meere. Sier Fanilien jino bef́ant. 


\section{Erfter 2(6)(h)nitt. Fిoologis. III. Bauctbiere.}

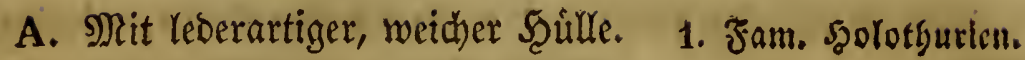
B. Nit Rareithaten und Ralefticken.

a. Reib Eugel: oder fideibenformig, ofne Sgauptfrablen. •. • 2. - Eeeigel.

b. Reib in megrere Sauptfirablen getbeilt. Sbne alfter, Maul unten. . . 3. - Seefterne. Dit einem Zlfter, Diaul oben. * 4. - Saarfterne. Erfte Familie. Selothurien, Holothurodea.

§. 107. eib mebre oser meniger lang geftrecêt, bismei= len wurmformig, von leberartiger Scillle umichloficn, aus

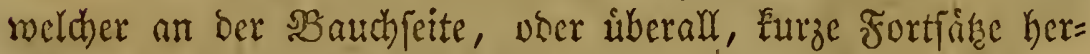
wortrcten, bie bas arbier fortichieben. Um bie vorbere Mund = iffinung weiche, cingiehbare, geiranjte 2irme. 2ffter am Scin= terende, bient zugleich als esingang in bas áftige unit vielen SBlafen befecte Sicpirationzorgan.

Holothuria tubulosa, wird gegen 1' lang, ift brann, bat am ¿and viele Gundert einziebbare șúfe uno findet fid im Dittelmeere.

3meite Familie. Secigel, Echinodea.

§. 108. Die aus vielen Saleftiden zufammengeichte Schale billot eine cinzige meiftens harbentgelformige Scullte,

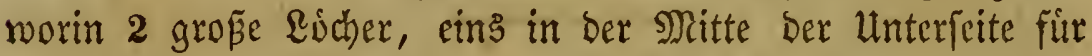

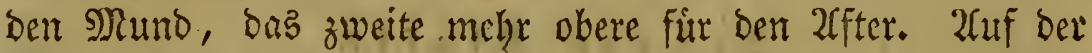
Schale viele Şucker, woran bewegliche Stadleln fiken; auserz

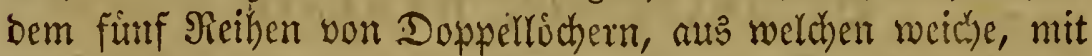
cinem Saugnapf endende Fitschen herborragen. Sein Res fpirationsorgan, fondern bas $23 a f f e r$ umipurt bie Srgane.

Der gemeine Seeigel (Echinus esculentus) Gat cine pomes ranzenformige Edale, nit fahmadjen Szickern, worauf die furzen, feinen Etadeln, und weiten Deffinungen in Den beiden solcu Der Sgauptadje. Sn Der unteren Niundoffinung ffeckt cin aub 5 3ábnèr gebildetez Siauorgan. Zlln europaif ftucké werden gegefiet.

Dritte Frmilie. Seefterne, Asterodea.

§. 109. Die Schale ift ebemfalls aus vielen Salffftuden zujammengefert, allein bieje grangen nicht genau aneinander,

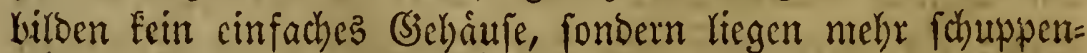
formig ibereimanber und find an ber weichen Scaut feffge= 
wachjen. Leib flad), fcheibenformig, in 5 - 25 Scauptarme getheilt, bie theils cinfach frob, theits fich fwalten ober gabel= formig zeráfteln. Sund unterl)alb; fein Nefpirationsorgan. Sein 2 fter.

Beim gemeinen Seeftern (Asterias rubens) ift ber flade $\mathbb{Q}_{\mathrm{cib}}$ in fünf ungetbeilte, breite Strablen ausgedernt, auf beren unterer Seite in Dor Ditte Die SintEfdilder feblen, Dagegen bier

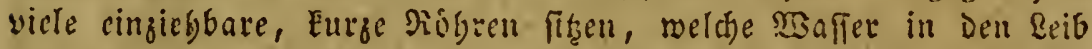

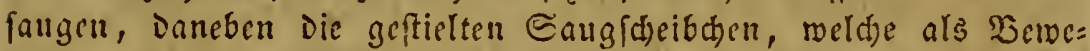
gungsorgane zum Siriecten bienen. Farbe rótblid. Siorojer.

Das MeDufcngaupt (Gurgonocephalus verrucosus) ift in 5 Seauptarme getbeilt, Deren jever fich wicierbolt fpaltet, fo Daff

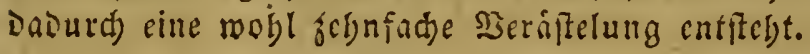

Sierte Familie. Şaarfterne, Crinodea.

§. 110. Sie haben die Seftalt ber lelstgenennten Gat= tung, aber Der Leib firt vermittelft anderer, ftroblich entiprin= genter Sitckenarme, ober cimes einfachen Stieles feff, fo baß bie Mumbiffinung nach oben fteht. Der 2after vorkansen.

Der gemeine Saarffer (Comatula mediterranea) bat begn Sauptfrablen um Den MRund, und Eleine RanEen am Riucfen oum Jeftralten. Mittclmecr.

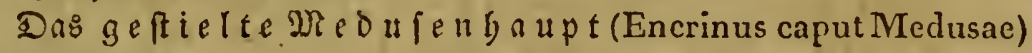
rubet auf einem langen, geglieverten, vom Diüsen entfpringenden

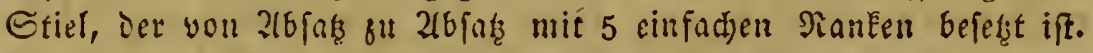
5 Sauptarme, Deren jeder gabelig iff, um Den 2lund. MBeftindifde Silffer, flist an Jerfen.

\section{EIf te $\mathbb{i}$ I a f f e.}

Ouallen oder Medufen, A calepha.

§. 111. Der gallertartige, meide \&eib biejer alfiere kit= Det theils eine Scheibe, theils eine groß̧e SBaje, theils einen

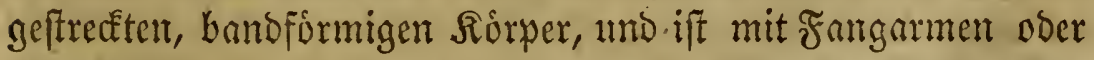
rangen, feineren, gleichfalts gallertartigen Fäben werjêten. Die Mundoffmung liegt bei ben icheibenfórmigen an ber Unterfeite, uno im Unmange berielben fitzen bie Fangarme, 4 oder 8 an 3ahl; bei anderen fehlt eine Mundiffmumg ganj, tho fatt ilfrer find vicle bohle Saugrobren ba, weldhe bie Mahrumg 
einnehmen. Diefen fehlt wohl ber Magen gallz, bie anderen haben einen großen, blafenformigen, mit Rebentafchen verfe= benen Nangen, nus weld)em äftige (Sefásée entipringen. Da rm und 2 fter fehlen. Seine Reipirationsorgane, aber bei vie= len SBlafen, welche burd) eigene Deffnungen $\mathfrak{L}_{\text {uft }}$ (Schwimm = blajen) ober $\mathfrak{B}$ affer ( Schwimmbihlen) eimmehmen jum Schmimmen. Die Suallen leben nebmlich) alle im Mieere, meiftens zwifchen Den aropen, haben cine hellbliulicthe, halb surchfichtige, bisweilen rithliche Farbe, wovon nur bie gelben :tm den Magen liegenden. (Fierft of ale alsgenommen find.

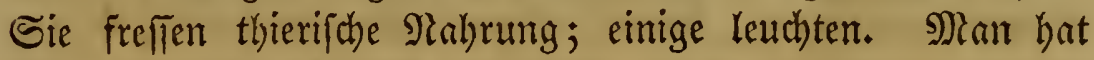

\section{3 Şruptgruppen.}

1. Mlit eimfacher Magenbóble.

a. Zluf Der Beibesfláde aus Bleinen Blátts den gebildete Rippen . . 1. Jam. Mippenquallen.

b. Seine Rippen, Reib fdeiben= oder glo: denfórmig, mit Jangarmen um Die Mitte Der unterflübe. . . 2. - Sdeibenquallen.

1I. Seine einjache Magenhoble, ftatt beren viele Saugróhren. Erfte Familie. Rippenquallẹ, Ctenophora. §. 112. Reib eiformig ober banbartig, Mundoffnung nach unten, fithrt in eine Şukle, bie eine jweite gegenibers ftehende Seffnung hat. Bei ben ciformigen laufen von einter Seffnung zur andern 8 Sippen fleiner $B$ láttchen, bie bei ben

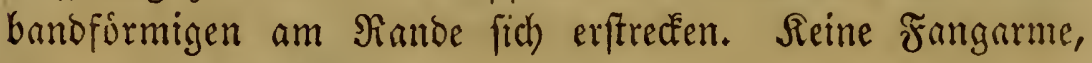
aber vft 2 Fangfíben.

Der $\mathfrak{B} e \mathfrak{n} \mathfrak{s} g$ il $\mathfrak{r} \mathfrak{c} \mathfrak{l}$ (Cestum Veneris) bat einen rangen, \{dimalen bandiórmigen Reib, in Deffen Ditte ocr lánglide Niagen; neben Dem Jiunde 2 Jangfáden. Bsefáfe Durdjlaufen den Reib. Snoifher Decant.

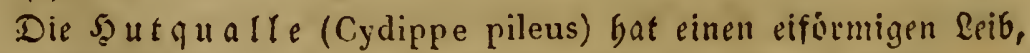
ein meites Maut mit mulfigem Miande, 8 Tippen und 8 Gefábe, Die yon einem Minge un Dell Miund ausgeben. Zwei lange Jangfáden. Zltiantifdes Meer.

3weite Familie. Stheibenquarlen, Discophora.

§. 113. Der fdeibenformige, runoliche $\mathfrak{L} e i b$ ift entweber,

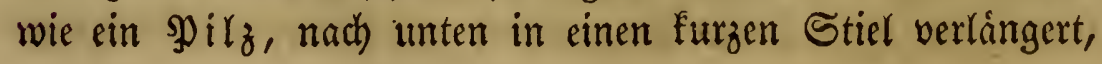


ober es fehlt biefer Etticl. In beiben Fållen bewegt er fïth burch) gleid)furmiges 3ufammenzieben uno barauf folgende flacke 2(usbreitung, wobei bie Sberíeite nach vorn gerichtet ift. 2un ber unterer Eeite ber Mund, von 4 oder 8 2rmen um= geben. Sn ber Mitte ber Magen, baneben bie Eierftode.

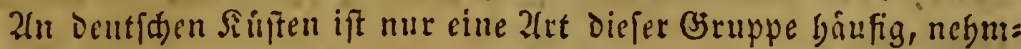
lid) Dic $\supset \mathfrak{h} r e n q u a l l e$ (IITedusa aurita); fie bat Eeinen હtiel, wier gefranjte 2lrme um Den פRuito, Dajwifden 4 Jabblen, morin Die 4 balbmonofirmigen Eierfácle. $4-5^{\prime \prime}$ im Durdmefîter. Doft: uno Siordiee.

\section{Dritte Familie. Siobrenquallen, Siphonophora.}

§. 114. Sie baben Eeinen einfacten Nund unt Na=

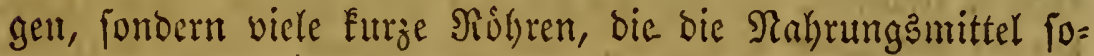
gleich) alsjaungen. Leib eine grope Blaje, ober aus vielen Eleimercit jujammenge|ęt. Sifine voer viele Sd)winmboblen. Lange Fillificioen.

Die Eecblafe (Physalia arethusa) Eeftebt aub einer grofen Brafe, Die wie eins fdrwimmente Ente geftaltet ift, anf Dem Riúden

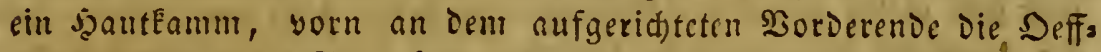
nung; viele Eurze Saugróbren an Der ß̧audfeite, Dagrwifden lange, Enotige Jáden. Lleber 1' lang. Zutlantifder Scean.

\section{3 mifte $\mathfrak{i} \mathfrak{t}$ a fle.

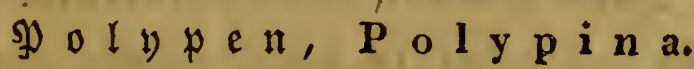

§. 115. \$hbiere von gallertartiger, weider, oft mut (c) leimiger Befchaffenkeit, mit Fugeligem ober cylinorifchem Sirper, all beffen vberent Ende cine Mundoffnung, von vie= Yen ober wenigen ( 8 ober 12), eimfact)en ober gefrangten, feit gemimperten Ientakeln ober Fangarmen unigeben, die

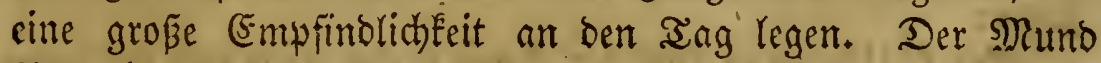
führt in einen einfachen stagen. Reine Reppirations = uno Eirculationsorgane, Éine Sierven, aber ziemlich beutliche Mus= ketn. Maniche Kaben Érnige Fierftode, bie in ben Magen munden; andere 'pflanjen fich burch Seimbildung fort, inben fie Sinozpen an ifyrem Rorper entwidfeln. Dieje Snozpen bleiben bei vielen mit bem Mlutterforper in \$erbinoung, trei=

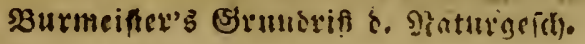




\section{Erffer 266 [d)nitt. Poologie. III. Bauchtbiere.}

ben mieber sinospen, und, to entfeht cin veráftelter, pflan zenformiger ahierftamm. SBei ben meiften fonbert fich an inm, theits an ber Sberflache, theils im Sentrum, Sarfmaffe

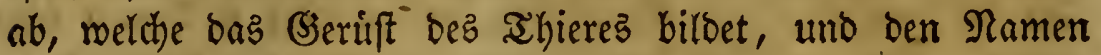
Sorallenft od führt. Die meiffen leben in seere beiper Şegendert.

\section{Erfte Familie. Polyactinia.}

Die \$olywen haben fehre viele Fangarme rings um ben Munb. Dabin:

- Die Secanemonen (Actiniae), mit weidem, aber Dod ziems

d) feftem, Galbengelfórmigem Sitrper, Der unten fid in eine breite Edfeibe ermeitert, mit meldher fich das Thier fefffę̧t. Maul obeu,

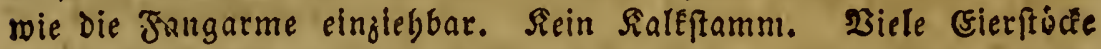
im umfange Des Magens iffnen fich in Diefen, und Die (Eier werden Durd Den Muno geboren. Fieine Sinospen uno bálftige TGeilung.

Der $\mathfrak{T}$ elÉ enEorall (Caryophyllia cristata) bat einen aufs

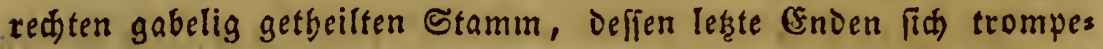
tenförmig zur Poigpenzelle erweitern; Diefe mit zaćigem Rande und febre vielen rabialen Eamellen. Sn jever Betle ein Polyp, Defs

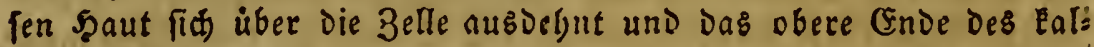
figen Stamms befleidet; Disfer bis $2^{\prime}$ bod und die 3ellen oft $3^{\prime \prime}$ meit. Sie balbiren fid, bilden aber keine Snospen. Sm rothen Meer.

3reite Familie. Dodecactinia.

Die Syolypen haben 12 Eurze, einfache ober gar keine Fangarme, und bilben unter ber gemeinjamen' Scaut einen Ealfigen Rorallenftock, welcher auf feiner ganzen Sberflache mit 4 fleinen meiftens 6ftrahligen (Srribchen bedectet ift.

Bei Madrepora bat Der Etamm einen zentralen, 6ftrabligen 2ldfentanal; er ift aufredit, veráftelt, uno zeigt yollig fernformige 3ellen.

Bei Millepora feglt der Sanal in Der 2laje und die Bellen fino blof runo. Die Jolypen Gaben keine Tentakeln.

\section{Dritte Familie. O ctactinia.}

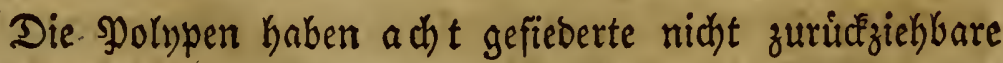
Xrme und eben fo viele binne Eierrácke, weldhe fich in ben

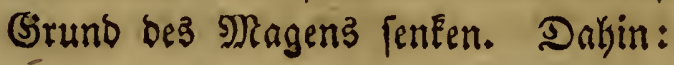


Der rotbe foprall (Corallium rubrum). Der rotbe, áftigs verzmeigte, auf Der Oberfiade fein gereifte Siorallfted mird yon eis ner weidjen şaut úberkeleidet, in welder 3ellen fir die einzeluen Đolvpen. Mittelmeer; liefert Die zu vielen Siunfffacten verarbeites ten rotben Siorallmafietr.

Der Dorde npolyp (Umbellularia grönlandica). 2l'm Ent De eines langen, imnen Ealeigen Etiels, Der im Doden Des Dieeres

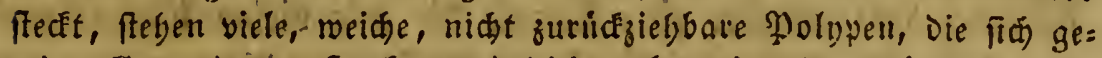
reizt alle grade ausftrecken uno didft neben einander orángen, fonft

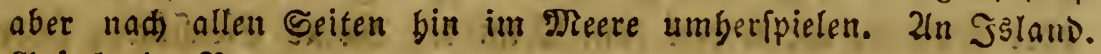
Brinland, siorwegen.

Dás Srgelmere (Tubipora musica), viele mit ciner ge: meinfdaftliden Safis feftrikęende Dolnpen fitcken ieder in einer Ealfigen Miobre. Diefe Rúgren didft neben einander, ourd Ealeige Suerfdiळten yerbunden. Jarbe blutrotb.

\section{Bierte Familie. Oligactinia.}

Die Solypen haben wenige $(5-20$, felten mehr) ein= fact)e, mehr weniger zurid ziehbare Fangarme; fie haben Eeine Eierfäcte, fondern treiben am Stamm Sinospen ober Sap: feln, worin Fier. Dalin:

Der $g$ rúne $\mathfrak{A}$ rmpolyp (Hydra viridis); ofne Edyale, eitr Furzer, Eolbiger Leib, libet mit feinem unterent Ende an -WBafferpflans ben feft, uno bat un Die obere Mindófinnung $6-8$ einfache, weit

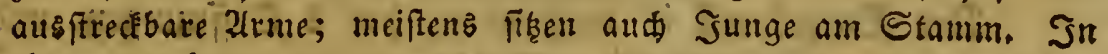

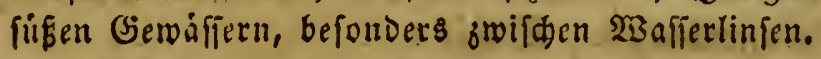

\section{Funfte Familie. Bryozoa.}

Die, \$ołypen baben viele fŕbenformige, nicht einziehbare

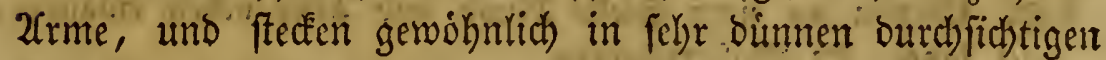

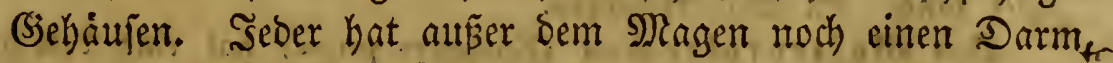
Der neben bem Munde fich offinet, unb einen Eierfoct hinter dem Magen. Dahin:

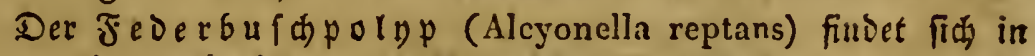

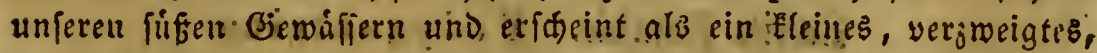
borniges $\Re 0$ gr, Das an allerlei Begenteanden, befonzers alten pfäbs len, fid himbindet, und alls ben offenen Enden der grobren Die balbmonofórmig geftellten, vielen, fein gefieberten Z(rime heryorffedt',

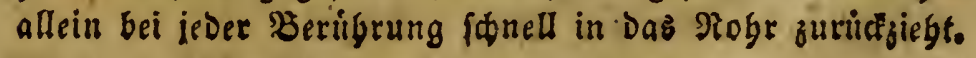


100 Erfter 2(bfonitt. Soologis. III. Bandthiere.

\section{Drefzegnte fiaffe.}

Snfufionzthiere, Infus oria.

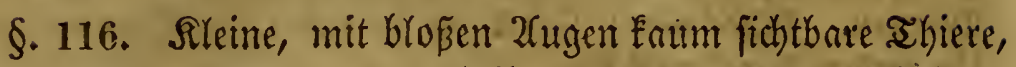
won rundricher, kltgel = voer feheibenformiger, ovaler Sieftalt, nit einfacher, von beweglichen Simpern umgebener Muno= offnung, won welcher ein. Sanal, ber mit vielen blafenformi= gen Erweiterungen befefst ift, entipringt. 2(fter vorhanben ober felyleno, oft neben bem Munde. Seine Eierftócée; ùber= haupt Eeine anderen imneren Srgane. Dieje Dibiere pflanzen fich Durch hálftige a sheilung, die entweder ber \&ánge oder Der Suere nach geht, fort; entfethen aber aud in 2(utginffen auf organifche Materien von felbft, baher ilyr Mame. Eimige ftehen, wie bie \$olnpen, auf cinem gemeinjthnftlicken Stamm, rofen fich aber hernach ab; bie Meiften find immer frei. Mran findet bieje albiere zll Millionen in allen, bejonders ftelyctioen, (Sewáffern, worin thierifche ober pflangliche Stoffe

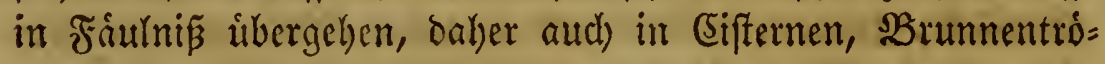
gen, Regentonnen, \&ofchfitbeln u. โ. w.

\section{Erfte Framilie. Enteropolygastrica.}

Der Darmfanal ift vorhanben, hat 2 Seffnungen, uno viele geftielte Magenblajen. Daijin:

Der $\mathfrak{O}$ I u menpolvp (Vorticella Convallarin); er beftegt aus cinem Eriedfenden Stamın, moran yiele yon langen Stiplen getras gene, becherfórmige Siórper, Die am freien Rande mit Eitien beféțt find. Ebenda an einer Etelle Minnd und Zffer neben cinander; $\frac{x}{24}$ "' rang. 2(n 2 Safferpflanzen.

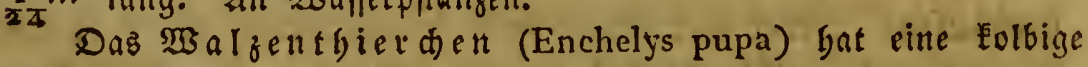

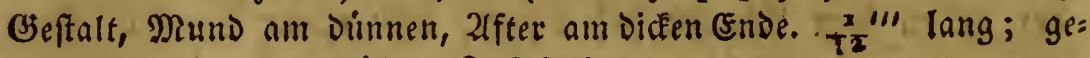
gemein. Eins Der gróften Snfuforien.

\section{3meite Familie. Polygastrica.}

Die İhierchen haben keinen \$arm, fondern bie gefficl= ten Mragenblajen gehen unmittelbar von Der Mtunoúffirung aus; ea' feblt alfo allen ber 2fftet. Dahin:

Die $9 R$ on a de (Monas termo) bat einen fegr Eleinen, rundi=

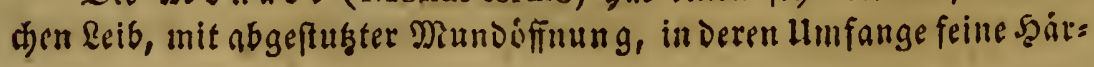




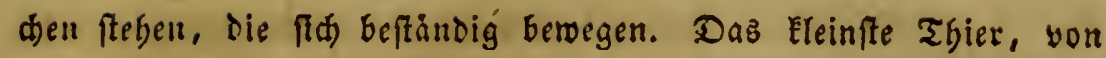

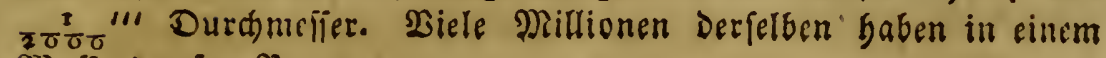

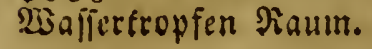

\section{Dritte Familie. Agastrica.}

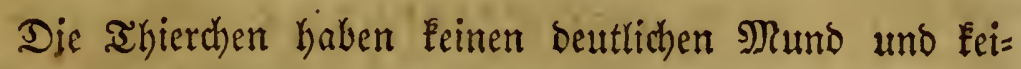
nen beuttlid)en Magen, Daker man fie niemals Slabrung zu fith nehmen' fieht; fie find $\mathfrak{k l a r}$, jeigen aber meiftens regel= mâpige dunfle Gitelle im Sinnern. Ware bewegen fich lang= fam uno unbelyolfen burd) 2tusftrecten einzelner Stellen bez Sörpers; oder SBicgung dellerben.

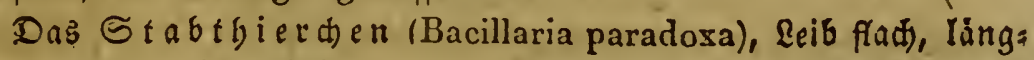

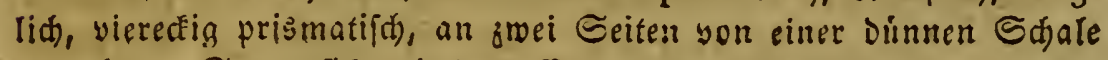
umieben. Eeţen fím mit Dem Ende oder Der Eeite fefr, und hán: gell reibentweiz an cinander. 


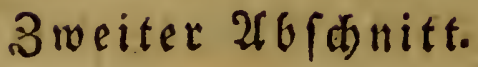

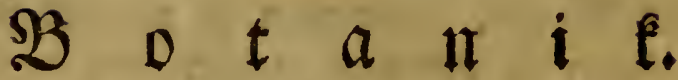

§. 117. Die B otánil hanbelt von ben in bie zweite.

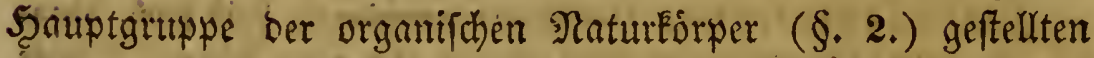
sigepen, von ben गু flanzen. Sie ift mithin bie Naturge=

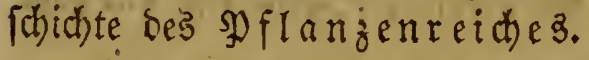

§. 118. D f fanzen (plantae) fino alle organifhen $\mathfrak{A}$ :

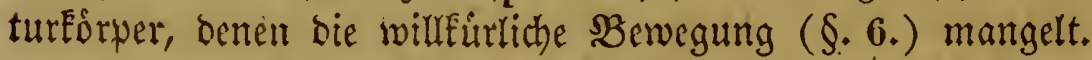

§. 119. Die \$yflanjen befteben fo gut wie bie IIfiere atts mehreren Srganen, welche bie Erhaltung jeder einzelneti

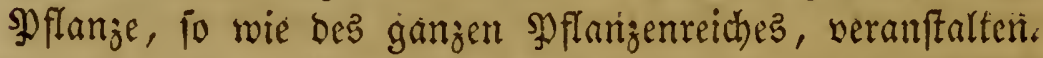

§. 120. 3mei Şaufen von Srganert haben bie Syflan= jen mit ben alyteren geniein, nehmilich alle bielenigen, welche

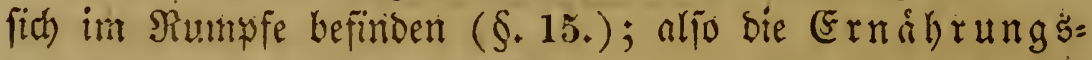
und Fortpflanjuriggorgane. Die ubrigen, b. h. Die

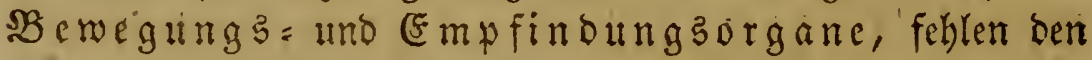

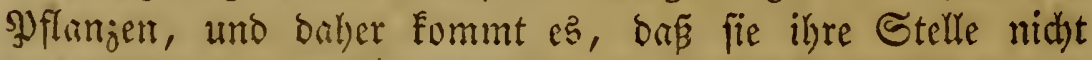
verlaffen, fo wie Eimorite von auken nidht ivabrnebment, ober fich Serfelben bewust werben fintmen.

§. 121. I. Die Ernahrungäurgane ber গ̧flanze finb:

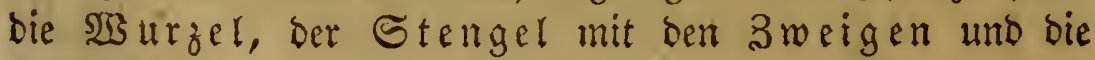
Bzlatter.

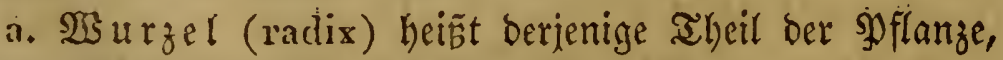
welcher nach unten in betn Soben binabfteigt, und bie Naly= rung ber פyfanizen eimfaugt. Sie hat baju Keine Deffnung, wie bie Ihtere Den \$lund, fondern auf Der Sberflache ber

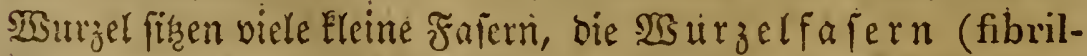
lae), welche mit inten tubigen, fichnamnigen Spizen ben Dlabrungsjaft sinjangen. Diejer befteft in reinem Maffer, ons 
aber jugleich Siohlenfäure, und bie Sáfte vermoberter Syflan= zen ober sabierftoffe, entí)alten muE; menigftens ift folches ISafier um vieles nahrhafter für bie \$yflanjen. Şiernach

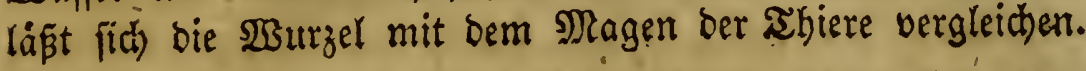

San untericheibet nach ber Form mehrere 2(rten bon

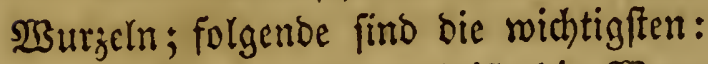

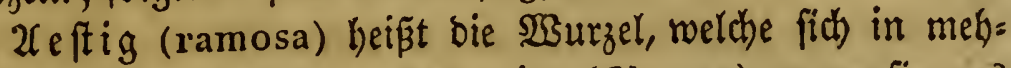
rere großße 3reige fpaltet; faferig (fibrosa), menn fie aus

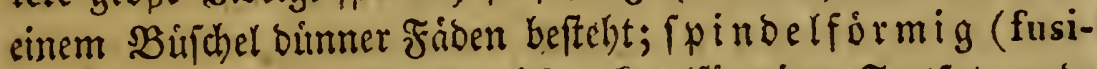
formis), wenn fie wie cin bicker, fegelformiger Fortfas grabe in bie (Erbe binabjteigt, z. $\mathfrak{B}$. bei ber Mobrrube; bismeilen

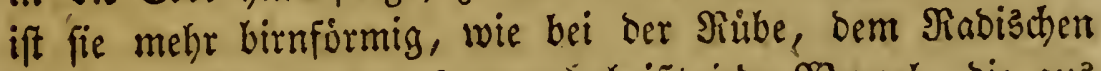
u. a. M.; Enollig (tuberosa) heiṕt jebe 253 utzel, bie aus ciner bid)ten, fleiid)igen, faftigen Mafle beftebt, an beren $\$$ ber

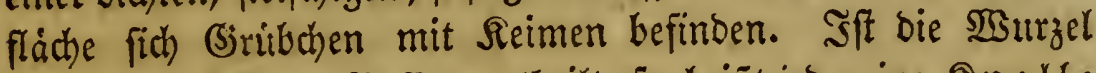
in mehrere folcher Maffen getheilt, fo heis̄t jebe cine Anolle (tuber). Die 3 wiebel (bulbus) unterideibet fich von ber Snolle baburch, baß fie aub mehreren Schichten beftelyt, unb nur einen Seim, bald in ber Mitte, bald am Sianbe, entrvicfelt.

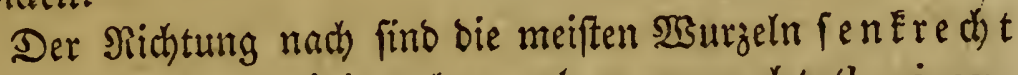
(perpendiculares), einige aber aud wagered)t (horizon-

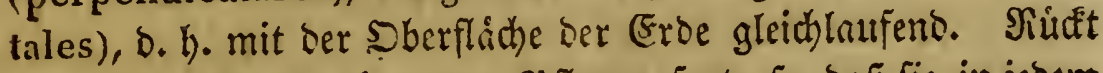
mit biejer $\mathfrak{W}$ utrzel bie ganze Pflanze fort, fo Daß fie in jebem Sahre. an eimer anderen Stelle ber Eroe vortommt, jo beift bie $\mathfrak{s u r z e l}$ Eried)eno (repens), j. B. bei ben Farren fráutern. -

Rad) bem 2(lter ber $\mathfrak{W} 3$ urzeln bat man folgenbe Ber ichiebentheiten:

einjábrige (annuae $\odot$ ) bie alle Saldr abfterben; zreijábrige (biennes $\delta$ ), bie im zmeiten sabre ab=

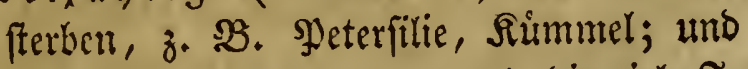
aแ弓b auernde (perennes $\nleftarrow$ ), bie viele Sabre alt werben.

§. 122. b. Der St a mm (truncus) ift berienize Itheif

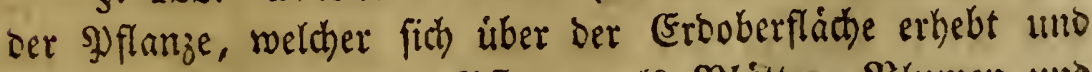

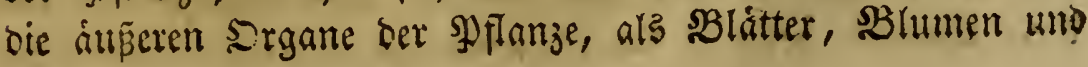


Fruchte trigt. Die 3 weige (rami). fino blope Fortiegult= gen beffelben, und ftimmen mit il)m im Ban uno ber $\mathfrak{B e r}=$ richtung iberein. Dieje befteht Darin, bie von ber şburget aufgenommenten Såfte in alle sheile ber Slflanje, und aus biefen verandert wieber zur Ş3urjel juruce ju füfren. Sonach

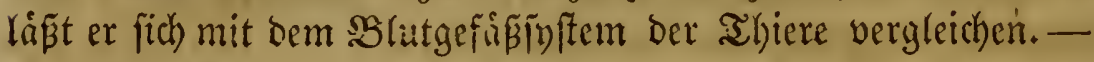

Der Sta!nm befteft, wie die ganje Sflange, ant vielen kleinen zwolfflachigen Bellen, bie in ber innigften Serbin= oung ftehen unb bas 3ellgewebe bilben. 3roifden diejen Sellen verbreiten fith (Sefá $\bar{\beta} e$, theits jerftreut, theils in Arei= fen um einander. Sn biefen liaft ber $\mathbb{S}$ aft (succus) dutrch)

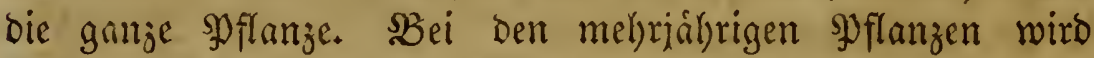

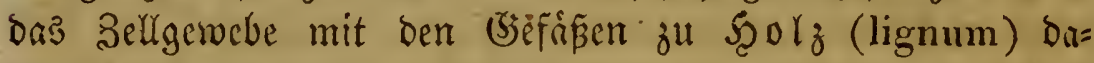
burch, baś die Safte aufforen barin fid) fortzubenegen, uno vielmebr eintrofinch. Um bns Şolz liegt dann nuß̈en am Stamm die siinde (cortex), roeldee eitre blos aus 3ell= getwebe beftebende Schitht bilbet, die fich alliblyrig meír ver= oict. Swifhen Sinde uno Scolz freigt der Saft auf, uno gebt theils in die Sinde, theils ins Scolz uber; auf bemiel = ben S13ege felort er auch wicber jurute. Das Einjaugen und ?uffeigen Des Saftes erfolgt bei unferen SBoumen zmal bes Sabres, nefmitich im 2cpril und Sitl; erfteres veranlä̧t das 'Uufbrechen des Enubes und der Shlithen, diefes die Reife ber Frutcht. "(ud) bildet fich bei ben mebriabrigen झlanjen aus bem libriggebliebenen Saft neues Sold (Splint), wel=

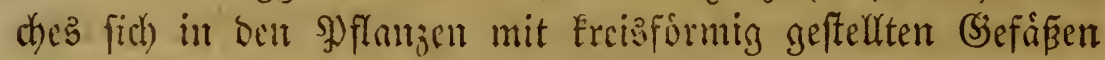

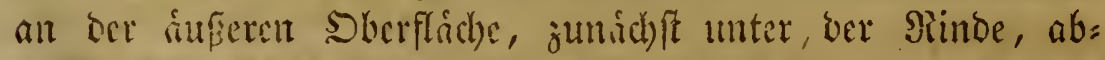

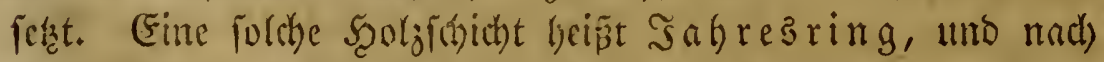
beren Ilenge kann man bas 2uter bes SBaumes oder 3wei= ges beftimmen.

Man untericheioet folgenbe Rrten des Stammes:

Sar boljige Stamm (truncus lignosus, voer bloź truncus) finbet fich bei ben $B$ atumen (arbor); fit aud a $r=$ tig (fruticosus) beifst ein holgiger Stanm, ber fid) gleich won ber Burjel in viele Jefte ipaltet. Fine SJflanje mit for d)em Strmm heist Strauch (frotex). 


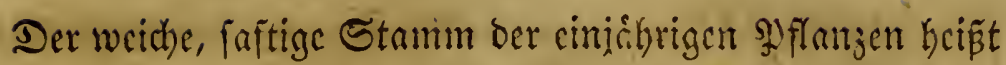
Stengel (cailis), und die ganje slffanje $\mathfrak{R} r a$ ut (herba); ift bicfer Stengel hohl, uno von 3eit ju Seit mit 2(njehwel= lunger, Inoten (nodi), verjelycn, an weld)en bie SBlatter fitzen, fo neunt man ir)r Şalm (culmus); triggt ein Sten=

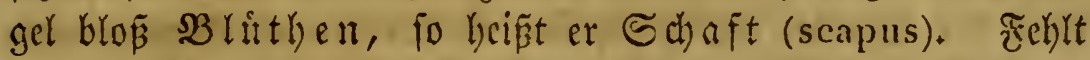
bei einer frautartigen Syflange ber Etengel, fo heißjt fie ften= gellos (acaulis); fried)t ein ummittelbar liber Der $\mathbb{N B}_{3}$ zel entfpringender Stengel am Boben fort und fhlight bann

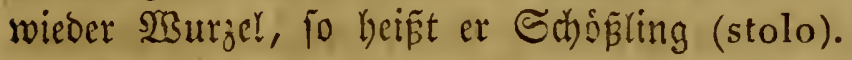

WBas bie 2(usbreitungen unb Fortiengungen des Stammes betrifft, fo nemut man bie gróseren $2($ efte (rami), bie fucine= ren 3weige (ramuli). SBeibe entipringen nut an folchen Stellen, mo fruber Blitter am Stamm-gefellen haben. Bleibt

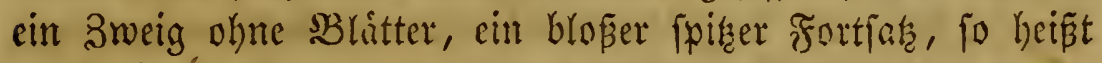
er Dorn' (spina); Der meiftenŝ Elcinere S tachel (aculeus) -

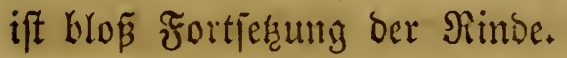

Nach ber Stellung und $\mathfrak{a} a g e$ unterfcheibet man ben Stamm wie folgt: aufrecht (erectus) wenn er jiemlich, und $g r a b e$ (strictus) wenn er ganz fenflecht ftelgt; $\mathfrak{a} \mathfrak{u}=$ fteigeno (ascendens) wenn er erft liegt uno bann grabe iff; liegend (procumbens, prostratus, decumbens) wenn er immer liegt; E Eiecheno (repens) wenn er liegt uno STBur= jeln treibt; wurjelno (radicans) wenn er an anderen $\mathfrak{K} \delta$ :

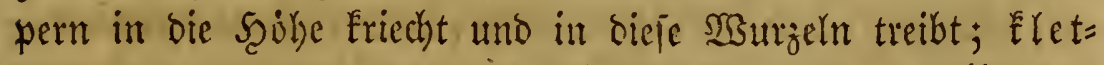

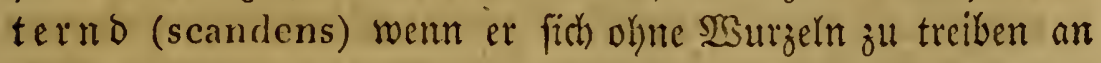
anderen Pflaujen aufrichtet; windend (volubilis) wenn er fich um andere splangen brel)t; ich marokeno (parasiticus)

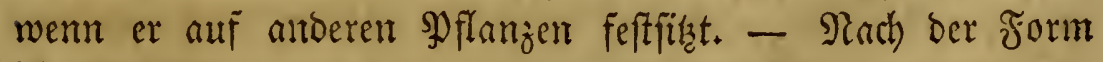
ift ber Stamm: runo (teres), oder halbruno (semi-teres), oder ecfig (angulatus), wothe bie $2 \mathfrak{n z a h l}$ ber finten noch nâlyer burch Sahlen beftinmt wiro; ober geflugelt (alatus) wern bie (Ecken in Yreite, hautartige $\mathfrak{E} a p p e n$ hervortre= ten; Enotig (nodosus) woun er. 2fmichwellungen hat, aber enodis, wo biefe fehlen; gegliedert (articulatus) went -er

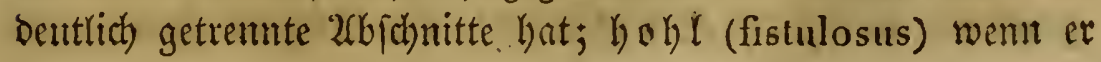


inmendig leer; fácherig (loculosus) wenn ber hohle Stamm burch Scheidewainde getheilt ift.

Da bie 3weige in Den BYattachjeln hervorwach/en, 10 frimmt beren Stellung mit sar ber SBlitter ukerein; fo get= ten auch von beioen gleiche 2fusbride, weshlalb ich auf Stel= lung ber Blätter nur himweife. Die erften Scervorragungen

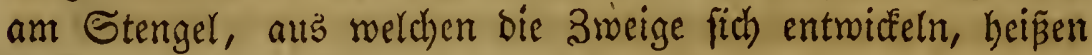
Snospen oder $\mathfrak{a}$ ugen (gemmae).

§. 123. c. Das $\mathfrak{B l a t t}$ (folium) ift eine 2fusbreitung ber গ̧flanzenjubftanz in bie Fláche. (5s beftelyt, wie Der Stamm, aus Ģefápen und Bellyewebe; jeme bilden bie Rippen (costae) oder $\mathfrak{B l}$ at tnerveat (nervi, venae), biejes bie dagmi= fchen liegende Blattjubitan. Die Farbe ber Blatter ift vor= zugsweife grin; ihre Serrichtung aber befteht barin, frifobe

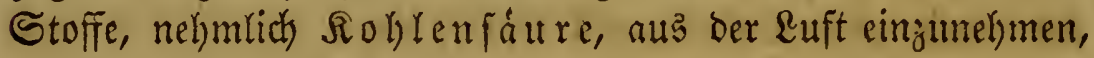
bamit biefe fich mit bem Safte verbinde; ein aryeil berfelben,

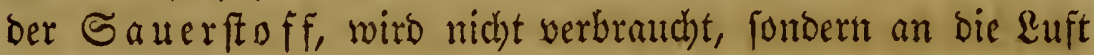
juritçgegeben. Diejer ganje Sergang erfolgt aber nur am Tage im Sonnenfochin, und if bem '2(t)men ber Thiere zu

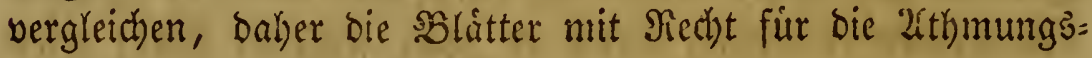
werkzeuge der şflanzen gelten.

Mant unterlcheibet ant iebent Blatte ben Etiel (petiolus), ober ben sibeil, mit welchent bas sBlatt an Stengel ficht, und bie $B$ lattflád $)$ e (lamina).

8.124. Der Stier ift theils rund (teres); theils ed ig (angulatus); theils rinnenformig (canaliculatus); theils mit feitlichen Scautlappen beriehen, geflingelt (alatus); theils

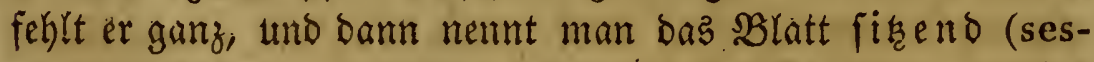
sile); reitent (equitans) beirst ein firgendes Blatt, welches

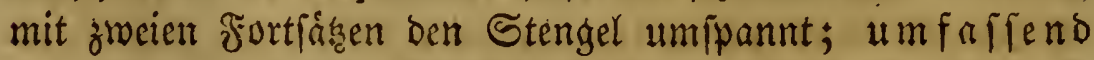
(amplexicaule) wenn fid) bieje Fortfátge um den Stamm ver= einet uno mit einander verwadjen; heráblaufend (decur-

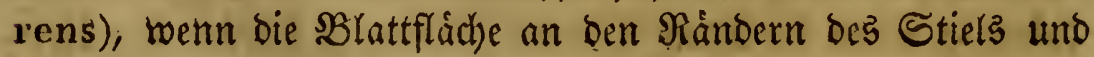
felbft an Den Seiten bes Stengels fith fortiefzt.

§. 125. 2(n ber $3 l$ a ttfla che untericheibet man bie Gie= getto, worant fich ber $2 B$ intftiel fegt, als (3) rund (basis) bas 
gegenuberffelbende Ende als Spifse (apex), bie Mittelfliche

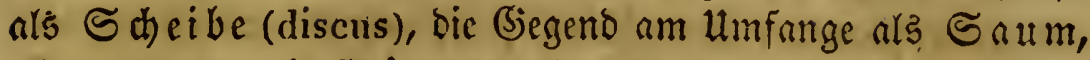
(limbus) und bie (Srinze Des Umfanges als $\Re$ and (margo).

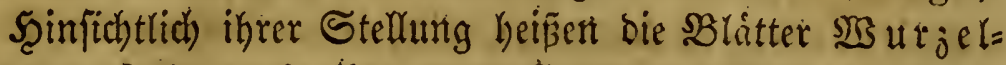

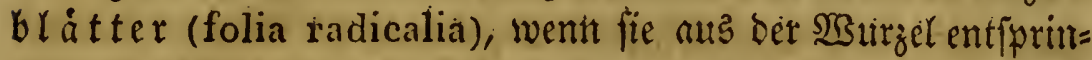
gen; Stengelblátter (f. caulina), wenn fie am Stengel, unb blithenftindige $\mathfrak{B l a t t e r}$ (f. floralia) wenn fie in ber Rábe ber siłume firken.

§. 126. Die Stellung ber Bhtatter am Stengel ift fehr verjobieden: gegentberfielyend (f. opposita) beipen fie, wenn fie in einerlet Szolke ant entgegengeferten Eeiten bes Stengels fitsen; falfchparige (dispariai), wemn folche ge=

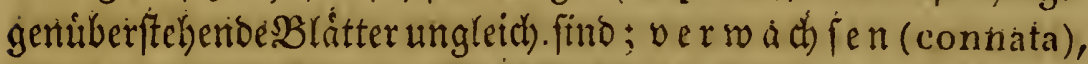
wern gegenuberftebente SBlítter Eeinen Stiel haben, fonoern mit ihrer Blattjubftanz zujammenhängen, fie beišst aud

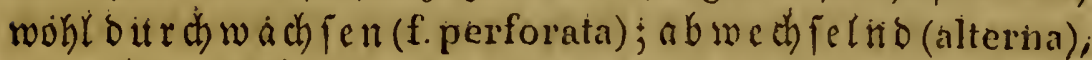
wenn in verichieberter Scoble an entsgegengefersten Seitert bes Stengelś; quitrlformig (verticillata), wenti in einerlé Scobe rings um ben Strumm zerftrut (sparsa) wenn obne alle Soronutrig; gebraingt (conferta), wenn fie bickt bei= fammen ftehen; fohuppenforming (inbricata), wenn fie einan=

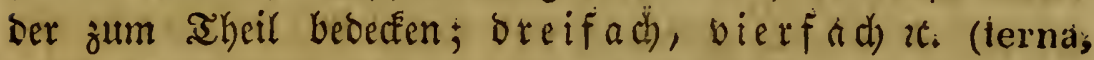
qiaterna etc.), werin biei ober viet SBlittet ringz um ben

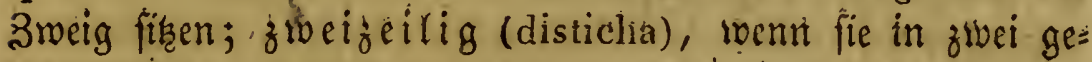
geniberftebenden Reihen fiken; jweiteihig, oreireihiget.

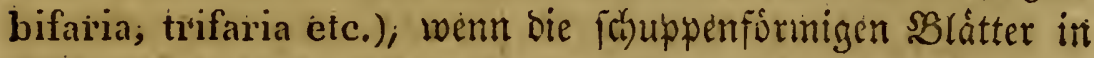
Beihen ftehen; angebriut (appiressa), wenn fie fich bidut

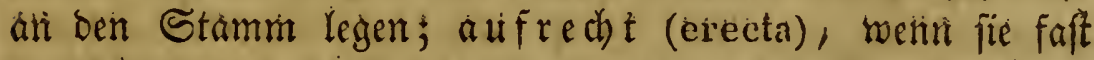
fentrectht ftefen; abftehend (patentia), wenn fie tuter einemi

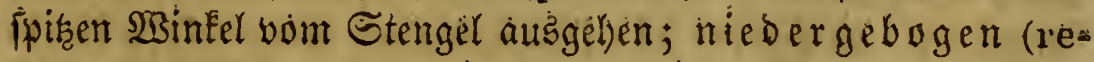
flexa), wenn fie mehr irach unten gerithtet fitio; eingerollt (ievoluta), wenn fie nach unteri ; a ufgetollt (involutio), wetin fie thad) obeti ziturifógerollt find.

§. 12\%. Der Form thad) werber bie Blattet eingethellt

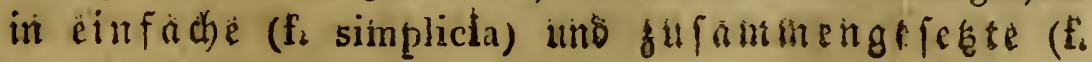
composita) 
(Finf a dbe Rlatter find biejenigen, beren Mittelrippe, als fortfetsung bes Blattfieles, ganz von Blattiubjtan um= geten ift. Solche cinfache $B$ látter jerfallen wieber int bie ungetheilten (f. indivisa), b. h. lorche, mo auch) ber Saum

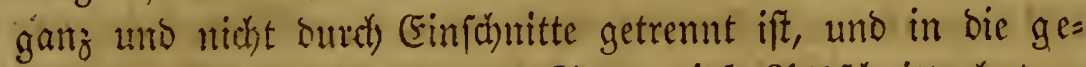
theilten (f. divisa), wo ber Saum tiefe 2ušfthnitte hat.

§. 128. (Finfache ungetheilte sblatter find: Das ruthoe $\mathfrak{B}$ Y. (f. orbiculatum), beffen Durchmeffer alle gleidh) fino; man mennt es fobirofor mig (peltatum), weenn fich ber SBlattfitel an bie untere Frache, nicht an ben (Sirunt bes BRat=

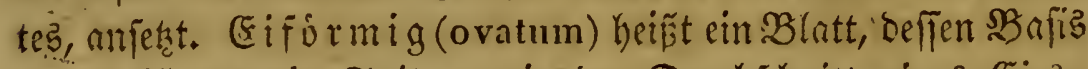
breiter ift als bic Spirze, wie ber Durdidnitt eines Fies;

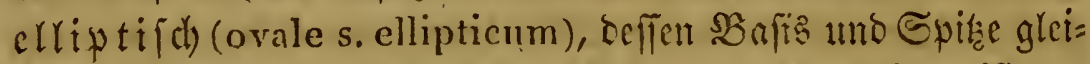
d)e Breite haben uno mo ber Langsourchmeffer gró berDuermefler; lanzettformig (lanceolatum), ein Kanglicheb

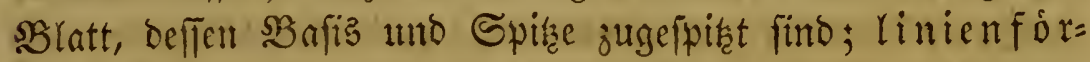
mig (lineare), cin lảngliches Blatt mit paralleten Rànorn; fc) werotformig (ensiformeł, cin langes, fchmales Slatt,

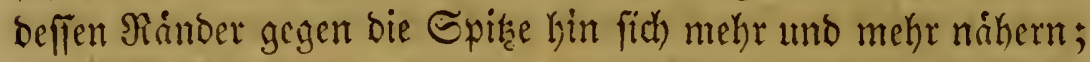
fdo alf felfurmig (spatulatum), ein Blatt, bas gegen bie Epike breiter wirb; herzfórmig (cordatum), ein breiecfines SBlatt, befien Seitemrånoer gefdhweift uno befien (Srunt auzgebuchtet ift' geigenfornig (panduraeforme), ein \$rott, befien Sei temrämoer tief nusgebuchtet fino; pfeilfórmig (sagittatum),

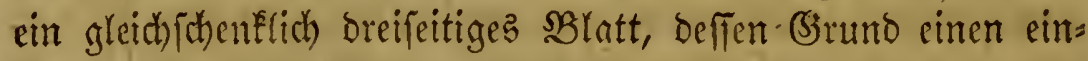
fpringenden SBinfer hat; w friemenformig (subulatum), cin etwas biffes, fchmales Blatt, bas allmaltig in eine Spike auslåuft; winfeligge Slátter (f. angulata), D. H. folche, derent

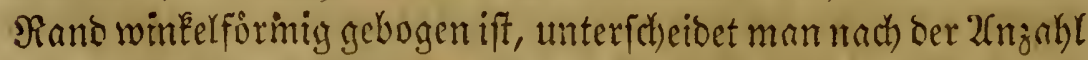
ber $\mathbb{B}$ Binecl, ars breifeitig (triangulare), vierjeitig (quadrangulare), fowohl quabratific) (quadratum) als rech t= ed $\mathrm{ig}$ (oblongum s. rectangulare), fúnfedfig (quinquangulare) H. F. w. (Ein breifeitiges Blatt, an beffen eine (Edfe (eigentlid)

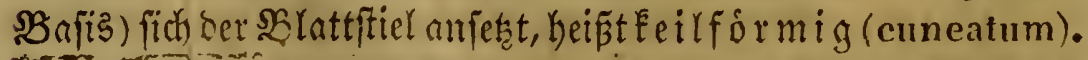

\$. 129. Die einfachen getheilten $B$ britter (folia simplicia divisa) Infen fich wieber nach ber Sithtung ber 
Einichnitte in 2 (5ruppen bringen, injofern bie (Finjonitte theils gegen bie Bajis, theils ienkred)t gegen ben Nitteluerven geridstet find.

Sur erfteren Form gelyoren: bas lappige Blatt (f. lobatum), mit (Finjonitten, bie blop den Saum theilen; man záhlt-babei bie Eappen und beftimmt das Blatt néker, als o $\mathfrak{b e} i=, \quad$ re $i=, \mathfrak{v} \in \mathfrak{e r}=2 c$. lappig (f. bi-, tri-, quadri-etc. lobum); das handformige $\mathfrak{B l}$. (f. palmatum), mit 5 ,

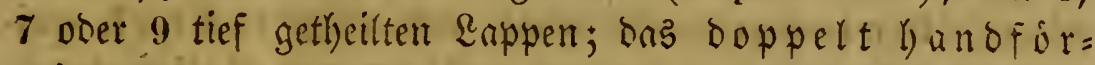

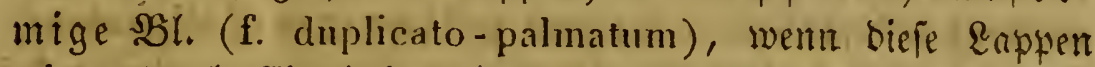
wieber burch (Finfchnitte getheilt find; facherformig (flabelliforme), wenn oas \$ßlatt keilformig ift, und bie (Fin= ichnitte mur ben abgeftufsten (Enorano theilen, aber tief find; bas z weithe ilige (f. partitum), ein $3 \mathfrak{B l a t t , ~ b a s ~ b e r ~ L a n g e ~}$ nach) geipalten ift; gabelformig (f. dichodomum), wenn

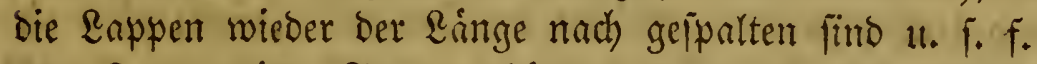

3ur jueiten Form geboren: bab $\mathfrak{b}$ uch tige SBlatt (f.

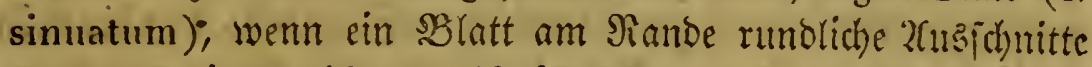
hat; bas eingeriffene (f. laciniatum), wenn umregelma= Bige (sinfchnitte oa fino; das leierfórmige (f. lyratum), wenn ein gegen die Epizze hin breiteres Blatt burch folche (Finfchnitte getheilt ift; fieberfpaltig (f. binnatifidum), wemn bas slatt paralletrandig ift und bie von ben (Fimfohnit= ten gebildeten sappen alle gleich find. Diefe aheilung wie= berbolt fich wohl an ben sappen, Dann heisen bie SBlatter, nach ber 3ahl ber ssteberkoltungen, boppelt, oreifach ac. fieberipaltig (bi-, tri-, ctc. pimatifidum).

§. 130. Die jufanmengeferten $B$ latter (folia composita), D. b. biejenigen, bei welchen ber Mittelnerv feine Brattiubftanj neben fich hat, vielmebr ben gemeinich af $t=$ lid)en Stiel (petiolus communis) Des ganzen SBlatte? bilbet, jeigen biejelben (Grundformen, wie- bie getheilten sirit=

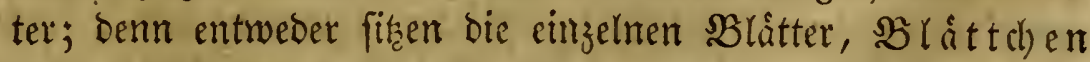
(foliola) genannt, alle am. Enoe bes gemeinjanten Bhatt= ftietez, Doer Der Reilye nach an beiben Seiten.

Unter bie erffere Form geljoren: bas gez we ite Shatt (f. 
binatum s. conjugatum), wo 2 gleiche SRlitter am Ende ঠes Gtiels firen, Das doppeit gezweite (bigeminatum), wo jwei Blatterpare am Fnoe des SBlattfieles ftehen, und das breif a d) geziveite (trigeminatum), wo brei fold)er

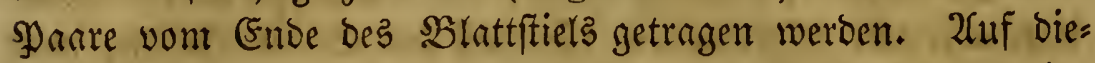
jelbe siseife giebt es cin einfach gedreites ober Dril= ling $\mathfrak{b} b$ latt (f. ternatum), ein doppeltes. Drillings= blatt (f. bi-ternatum) und ein oreifaches Drilling $5=$ blatt (f. tri-ternatum). 2uch) das funffache (f. quinatum) unb fiebenfache ober gefingerte (f. digitatum),

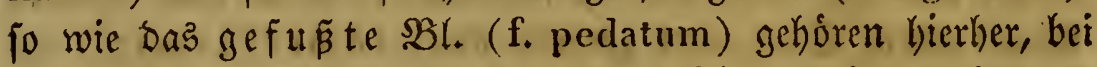
welchem lesteren ber am (Enbe gabelich) getheilte Stiel am Snmenrande ber (Şabel 5-9 Bliattchen trágt.

Unter die zweite Form Eommen alle gefiederten $2 B$. (f. pinnata). Schlechtweg gefiedert beift jebes Blatt, bas gegenuberftebende BRlitter am Scauptftiel trigt; jebes die= fer Blattpaare Keipt Soch (jugum), uno nach ber 2anzahl ber Soche das Blatt z weijochig (bijugum), breijochig (trijugnm) u. 5. w. Steht ein umpaares SBlatt am Ende, fo beipst es unpaarig gefiebert (impari-pinnatum), no nicht, paarig gefiedert (abrupte-pinnatum s. pari-pinnatum). Sft ftatt bes unparen (Endes ein fpiralig gewun= bener Faben, eine $\mathfrak{R}$ anke (cirus) da, fo heipt das sBlatt ranteno gefiebert (cirroso-pinnatum). Sheilt fich ein SBlattfiel gabelformig, uno billoet jebe Binke ber (s)abel ein gefiedertes Slatt, fo heipt bas ganze Blatt verbunden ge= fiebert (conjugato-pinnatum). (S) fingert=gefie= bert (digitato-pinnatum) ift diejelbe frorm, aber ons Ende Des Stieles liaft in mebr als 2, meiftens $5-7$, Nebenftiele aus. Sind die Blattchen eines gefiederten Blattes wieder gefiedert, fo heist das ganze Blatt doppelt gefie dert (bipinnatum), aud breifach und vierfad). (tripinnatum, quadripinnatum) gefiederte Slatter fommen vor. Nod) mebr gefiederte Blâtter, Deren Blattchen gewobnlich fichon ungleiche (5roje und 3ujammenjekung baben, nennt man iberzufammengefét (supradecompositum). 


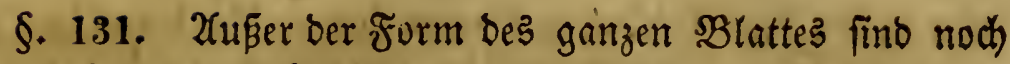
die verfchiedenen 2Ubinderungen des Grundes, Ranbez, ber Spige uno Brattfläche von $\mathfrak{W B}_{\text {Sidhtigleit. }}$

Der (j) uno heíst abgeftukt (f. truncatum), wenn er Durch) eine grabe Linie begranzt ift; ausgerandet (emarginatum), wenn er an ber 2(nheftungşftelle bes sBrattfitiełes

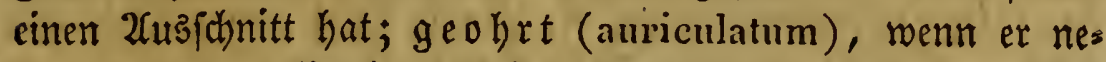
ben bem Blattftiel in 2 Eleine, runde $\mathfrak{L} a p p e n$ ermeitert ift.

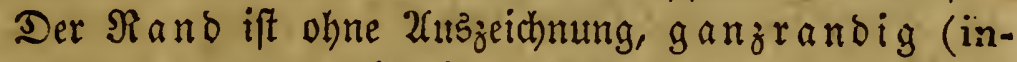

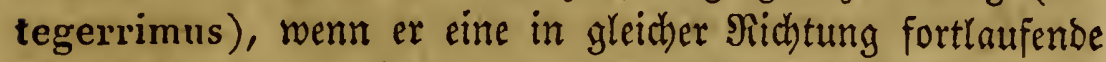
Linie beidyreibt; gezahnt (dentatus), renn er (Einfdhitte hat bie ftumpf fino, bie Bályne bagwifchen aber fpir; gekerbt (crenatus), wenn bie (Finjohnitte fpis, bie Záhne ftumpf fino; gefagt (serratus), wo beide fpis find; wimperig (ciliatus), wenn feine Saare am ganjen Rande fiken.

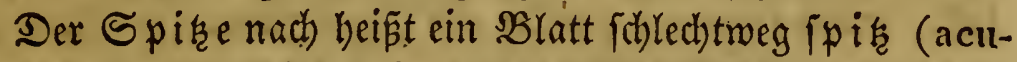

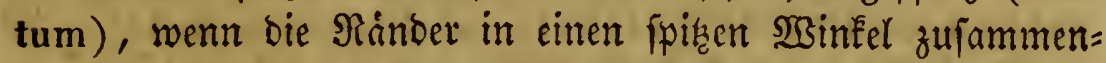
ftopen; zugefpift (acuminatum), wenn biefe Spifenod) be= fonder' hervorgezogen ift; feinguge fpitst (cuspidatum), wenn bie verlángerte Spike fehx lang ift; bold) fpicig (mucrona-

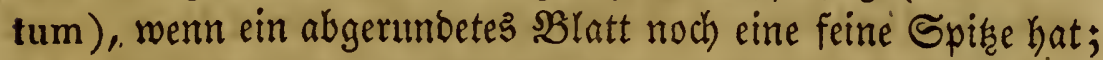
ftumpf (obtusum), wenn die Spife abgerundet ift; abge=

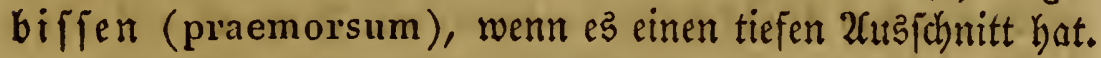

Die Serfochedenheiten ber Fläche rikgren theils von ber SBlattfubftanz felbft, theils von fremben barauf rubenden ahei=

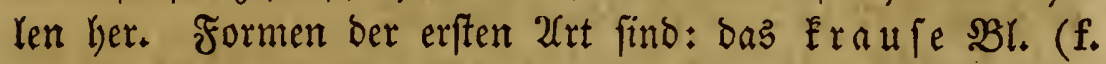
crispum), wenn ber Rand weiter ift als bie Flóche; Das runjelige (rugosum), wenn bie Blattjubjtanj zroifchen ben Rippen fich) erkebt: gefaltet (plicatum), wenn es ber Rán= ge nach in Falten gelegt ift; ielfírmig (carinatum), wenn bas Blatt ber $\mathfrak{L}$ ange nach zufammengejeblagen ift und nach unten aljo einen Siel bildet; glatt (laeve), wenn pol= dhe Şerjachicoenheiten fehlen; glà nzeno (nitidum), went bie Blattfladbe fpiegelt.

(Fin Shlatt, auf weld)em keine Scaare ftehen, heift $\mathfrak{b}$ a ar= ros (glabrum); rauh (scabrum), wenn es mit fehr Eleinen 


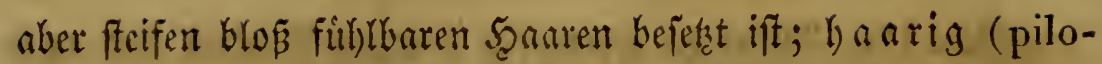
sum), wenn auf ber Sberflache einzelne lange Scaare fellyerr; zottig (villosum), wenn es fel)r lange bifchelweis groringte weidhe Saare hat; weid)lyarig (bubescens), wenn bie Scaare Flein, weid) unb frin firto; feiben artig (scricenm), wem bie Eleimen Scanre sicht antiegen umb feisenartig glingen; wollig (lanatum), wenn bie Scaare lang uno gleichformig bidit, auch wobl gefraujelt find fil jig (tomentosum), wern bie Szane jebr bicht fteleen und feft in einander verwebt find; beft áb t (farinosum), wenn ein mellartiger Staub auf ber Sberfliche liegt, pollinosum, menn biejer Staub gelb ift; bes reift (pruinosum), went eill weiz̈er Şauth die Sberfliche be= fleibet; $\mathfrak{b}$ fchuppt (lepidotmm); wenn Eleime Schuppen auf Der Sberflicke liegen; war $\mathfrak{j}$ ig (papillosum), wenn bas \$latt Eleine, fleijhige $2 \mathfrak{s a r g e n ~ h a t ; ~ o r ~ i ~ f i g ~ ( g l a n d u l o s u m ) , ~ w e n n ~}$ belle, etwas crhabene \$punfte mit cincm id)warzen Mittelpunft auf bem Slatte ftehen; Elebrig (glutinosum), wenn ein im

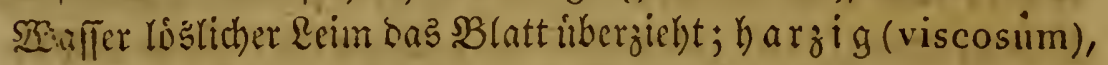
ment biejer flebrige Uebergug im $2 \mathfrak{s a f f e r ~ u n l o s l i c h ~ i f t . ~}$

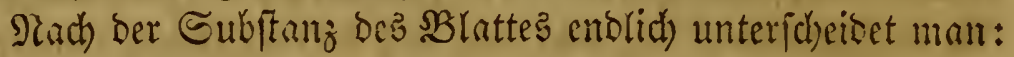
bas há utige $\mathfrak{B}$ l. (f. membranaceum), wemn es bium und feit ift; bas lederartige (coriaceum), wenn ez fteifer utho bicker ift; bas fleifhige (carnosum), wenn ez aus cinem biffen, faftigen Bellgemebe befteht; bas ho ohle (fistulosum),

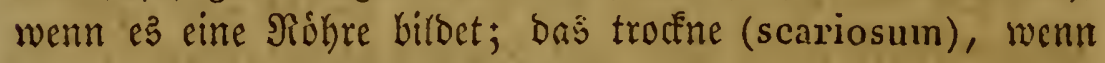
es ganz ober jum :iheil vertrocinet ift.

§. 132. II. Die Fortpflanjungzorgane Der (Gies

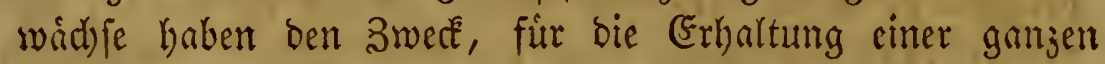
\$ুflamzengruppe Sorge ju tragen, uno Reime zu bilden, aus weldyen, wenn bie alten \$flanzen abfterben, juntge \$y flanzen

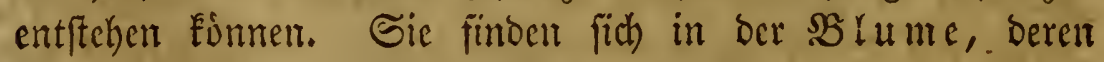
Baau wir alio junichit unterjuchen múfien.

Eime vollémmene $\mathfrak{B l u m e}$ (flos) befteht aus mehreren Srreijen won $B$ láttern, die att einer 2tre übereinanoer befeftigt fino. Dieje 2(re, aljo auth bie ganje SBhume, wiro vom S̊lumenftiel (pedunculus) getragen. Meiftens erweitert 
ex fich Folbenförmig uno bilbet eine Enoflache, auf welcher bic Blume und fpáter bie Frubt rubt; man nennt bicfe Fläble beshalb frudbtboden (receptaculum).

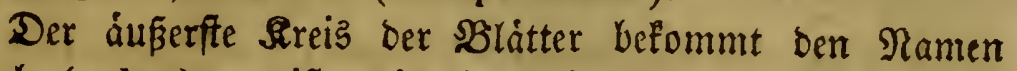
Selch (calyx); er ift, wie bie Blatter, grin gefórbt, uno firmmt mit ibnen auch in ber Jat bes Ueberzuges úberein.

Der zweite Stris, bie $B$ lumenErone (corolla), un= tericheibet fich auserbem burh bie bunte farbe vom Reld); die Blätter ftehen in ber 'siegel fo, Daß̈ fie bie 3mifchentállme zwifhen ben Reldbblättern ausfüllen.

Der britte Areiz ift reniger blattformig. Er befteht aus

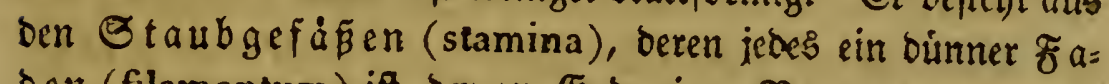
Dert (filamentum) ift, Der am Ende einen $B_{e}$ utel (anthera) trágt, in weldbem viele feine Rornchen, bie $B$ I umenftaub

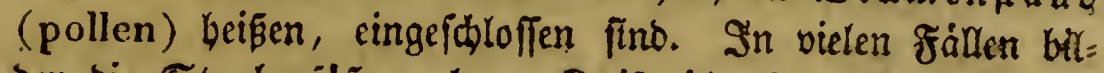
Den bie Staubgefäáze mebrere Rreife ubereinanber.

Die Ire ber Blätter, oder ber Stemper (pistillum), if cin cin = ober mehrfacher ftielformiger fortfak, ber un= ten sincu fltopf bildet, weldher $\mathscr{F}$ ruch ten oten (germen) beist, weil aus ihm bis Frucht entfeht. Diefer zruchtenoten ruhet allemal auf bem gruchtboden uno beftegt aus bem vier=

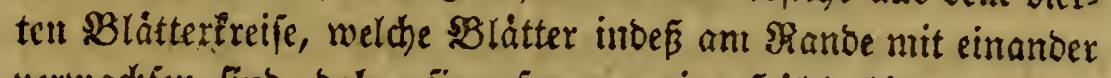
verwad) fen fins, baher fie zufammen eine sobjle bilden. Bon Der Spitse bes ôtruchtenotens entipringt ein büner Fortfak, ber (S) riffel ooer Etaubweg (stylus), weldher nach oben! in cinen Eleineren senopf, oder in cinen oder mehrere gáben aubláuft, bie man $\mathfrak{N a r b e n ~ ( s t i g m a t a ) ~ g e n a n n t ~ h a t . ~}$

Dies find bie \$̇jeile einer vollfommenen Blume. Scả = fig fehlt bie Blumenirone (fl. apetalus), und bann ift ber Reld) allein vorlyanden. In biefem suftande beist er folled)t=

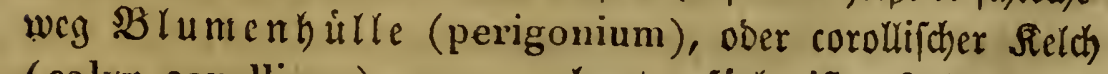
(calyx corollinus), went er bunt gefarbt iff. Feflen beibe Slifterfirife, fo heiṕt dic Blume nacft (fl. nudus); feblen. blop die Staubgefápe, fo heift fie weiblid) (fl. femineus), fehlen blop bie Gtempel, fo nenut man fie mannlido (ft masculus); fehlen entolich beibe inneren Iheile, bie Staubge

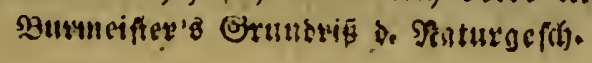


fäpemitoen Stempeln, fo beipt bie ßlume un $\mathrm{r} u$ db tbar (stezilis); fino fie vorlyanden, fo wiro fie in SBejug auf die 2 nocjenteit Diejerabeile 3 wit ter b f $m$ m (flos hermaphroditicus)gentannt.

§. 133. Die Stellung ber Blumen, welche man mit bem Ramen des Ş zeigt manche Derfehivenkeiten. Uripringlid fiteht eigentlich jese SBlunte in Der 2ch/el eines SBlattes, D. G. Da, wo bas SBlatt am Stengel fikt, ad) felft in noig (axillaris); boch fann biefes BBlatt nud) bas letste am Stengel fcin, uno bann ftebt bic SBlunte auch am Enbenes Stengels, gipfelft a no ig (telminalis). Das şlatt, welches unter ber Şlume fteht, verândert gemeiniglich feine Form

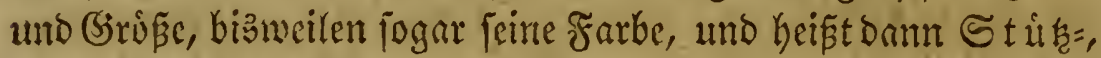

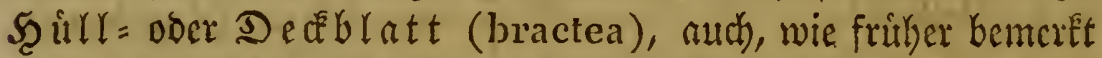
wurbe, blumenft ánoiges slatt (fol. florale).

Srigh ein Blumenftiel nur eine SBlume, fo heipt $\mathfrak{e x}$ ein blu mig (p. uniflorus); mebreresßumen záflt man, uno benent ben Stiel banach ; we $i=$, orei=vier $=3 c$. und vielblu= mig (bi-, tri-, quadri - etc. multiflorus). Sisen an einem viele SBlumen tragenden Stengel Die einjelnen SBlutben nuf bejonderen Stielen in Reiben und frei, fo beist der SBlithen= ftand eine $\mathfrak{x} \mathfrak{i}$ ube (racemus); ftehen die Slumen dicht ge= oringt und ungeftielt in Reiben am Enoe bes gemeinjamen Stieles, fo heifst der SBluthenftand eine Uehre (spicá); die Sieifen nennt man 3 eiren (stichis), und barnatb die 2ebre ein $=$ jwei= 2c. zeitig (mo no-, di - etc. sticha); ftehen an jeber Stelle nicht einjelne SBlumen, fondern wieber viele, nuf

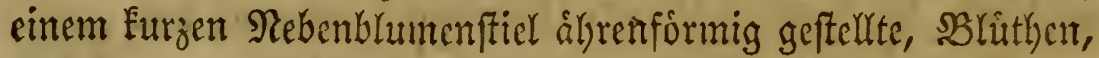
fo nennt man biefe $\mathfrak{X} e \mathfrak{r c h e n ~ ( s p i c u l a e ) ~ u n o ~ d i e ~ g a n j e ~}$ Zehre jufammengereft (sp. composita); ber gemein= fohaftliche Stiel beifft die 2 re (axis s. rachis). Sft ber ge=

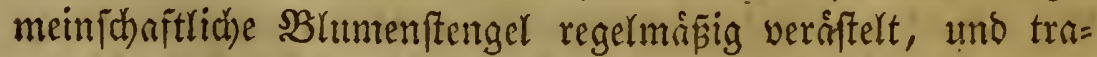
gen bie Nebenftiele mebreve Blumen ober 2ebrchen, fo nennt

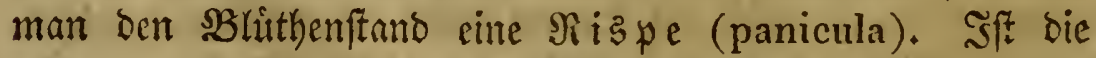

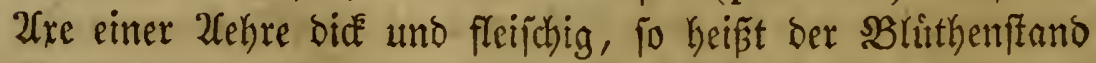
Rolben (spadix), uno das grobe Scillblatt am Sirumbe des

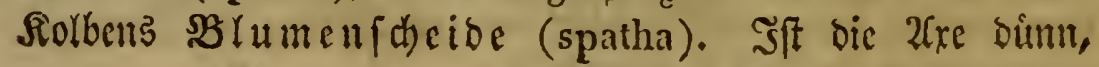


s. Hunidus) u. f. w. Der cinbláttrige Selch ift am Rande meis

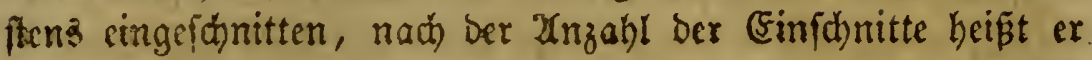
eins, zroei = x. jáhnig (uni-, bi-etc. dentatus); finobiefe

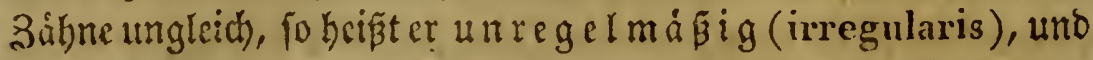
birben die ungleichen 3ábue zwei gegenuberftehende Sauptlap = pen, fo wirb er zroeilippig (bilabiatus) genannt.

Lie 1 lumentroute betreffend, fo ricberbolen fich bei ibe faft alle formen oes Seldes. Sie ift, wie jener, ballo einblattrig (corolla monopetala), balo mebrbláttrig (c. polypetala); jedes Bintt heifit Blumenblatt (petalum). Steht fie frei unter bem fruchtenoten, fo beift fie c. hypogyna, vermadift fie mit bem seldh e. perigyna, fteht fie mit Diefem auf bem fruchtinoten c. epigyna.

Die einblattrige Blumentrone zeigt alle genannten for men beş Relaez. Bei biefen Formen untericheibet man ben unteren verengten sheil als Robr (tubus), uno die obete, meiftens in Lappen getheilte, 2ubbreitung alb $\mathfrak{S}$ r. $\mathrm{m}$ (limbus); too beibe an einonber gränzen ift ber (singang, $R$ as c) en (faux) genannt, weldher bismeilen von fleinen Schupp (b)en, (S) wo olbe (fornix), gefdloffen (clausa) ift, báufig aber aud) offen fteht (pervia). SR a b fórmig (rotata) beift cime einblättrige SBlumentrone, bei melcher Siobr uno Saum alb målig in einanber ubergehen uno jiemlich flach ausgebreitet fino; práfentirtellerfórmig (hypocrateriformis), wo bas Sobr febr lang und eng, ber Saum aber breit uno plótzlich flact) außgebreitet ift. F a benfórmig (c. ringens) nennt man bie Blumentrone, wenn fie 2 Sa upltippen (labia) hat, unb mablirt (personata), menn ber Fingang ciner fold)en burd bie gewolbte Unterlippe gefdiloflen ift; gefpornt (ealcarata), menn fie am (Stunde einen lángeren ober fúrzes ren hobien fortfab, Sporn (calcar), hat; oungenformig (ligulata), wenn fie nad) einer Scite bin in einem flachen, blattformigen fortias ausgebehnt iff.

Die. Staubgefáze zeigen rocnige Nerfchiebenheiten. şismeilen fehit Der Faben, Dann beißst ber Beutel fisend (anthera sessilis), báufiger fehlt ber Beutet, onmin nennt man 
ben Faben unfrud) $t$ bar (f. sterile), ber Faben ift meifenb runb (teres), bismeilen flach, blattartig, ober edig uno lanzettformig, meiftens if er einfach, mitumter ver= zweigt; in ben meiften Fallen fibt an ilym Der Bettel mit Dem einen Ende feft (a. erecta), feltener mit ber Eeite, bin = gend (a. pendula). Der Beutel beftett aus 2 Fithern (loculi), bie burdh eine Sdeibewand getrent fint. Diefe fådber vffnen fich bald mit $\mathfrak{R l a p p e n ~ ( v a l v u l a e ) , ~ b a t b ~ j e r = ~}$ reifen fie ' (dehiscentes).

Der Stempel if unter allen Srganen bar Bhume daz= jenige, melhes die meiften \$erichiebenheiten zeigt. So ift bie Narbe balo enopfformig (capitatum), bald fabenformig (filiforme), bald einfach (simplex), baro in mefrere gleiche Fortföke gefpalten (fissum), beren 3ahl nảber angegeben

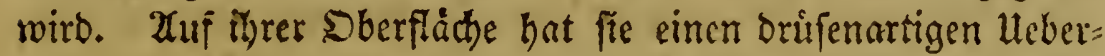
zug, an melchem bie Blumenftaubforncthen fefteleben, ifren

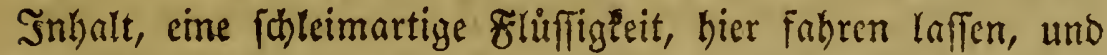
Daburd) bie (Entwickelung Der Blume zur Frutht bebingen;

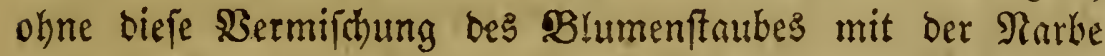
entfteht leine Frucht, uno bie mangetnoe Entwidelung fo vieler Blumen bat hierin ihate Urjacthe.

Der Staubroeg feblt vielen Blumen, unb bann fitst die Rarbe unmittelbar alf bem fruchtfnoten; in anderen

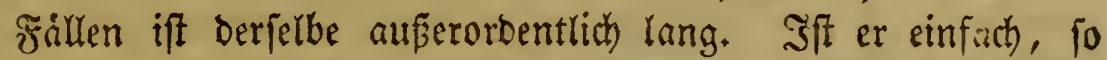
bat er in ber Mitte einen Nanal, ber in bie Scible bes Fruchts fnotens fitbrt; ift er gefpalten, fo liegt ber Fingang in ben

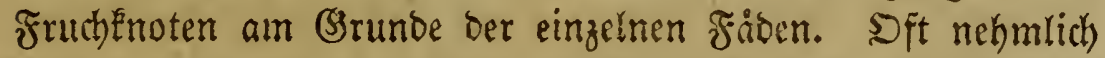
ninment er an Der Berpaltung Der Tarbe Zntheil, unb iff bann

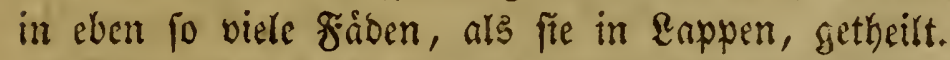

Der Fruchtenoten beftet aus 4 abeilen: 1) ciner

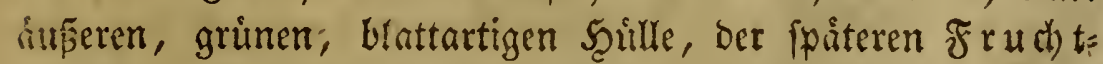
bulte (periearpium), unter melcher 2) bie fieime ber $\mathcal{S}_{a}$ men, bie (Eierchen (ovula), enthalten find. Bermitterft cines Föbdens, 3) bě $\mathfrak{R}$ abelftranges (funiculus umbili(alis), fitsen fie an einer verbiffen Stelle ber inmeren beoble

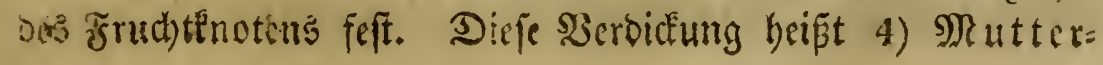


fucken (placenta), und befindet fid) da, wo bie sBlátter ber

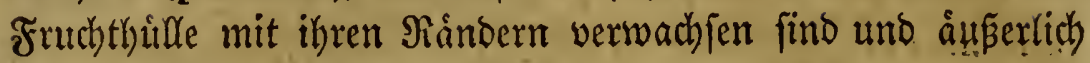
cine Falte, cime 2 rt von $\mathfrak{N h}$ t (sutura), bitben.

§. 135. In vielen Şlumen bemerft man, auß̧er ben genonnten aheilen, noch eigene brifjenartige Sivrper, weldhe, weil fie Şonig abjonoen, Şoniggefå äe (nectaria) genannt werben. Sie fisen oft an anderen ahbeilen, j. B. ant Relch), ober an ber-Błtmentrone, befonder' im Sporn; in anderen Fillen fteken fie frei, innerbalb ber Bhtumentrane, zwijd)en ibr und ben Strubfíden. Rinne bezechnete úbrigens mit

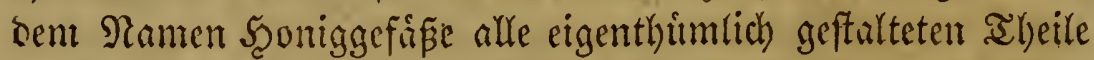

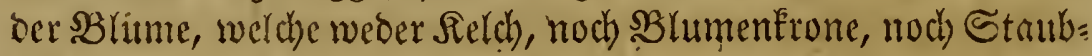
fioden, noch Stempel find, ferbft bann, wenn fie gar feinen Şonig abjonoern, und vicle $B$ otanifer fino ihm barin gefolght.

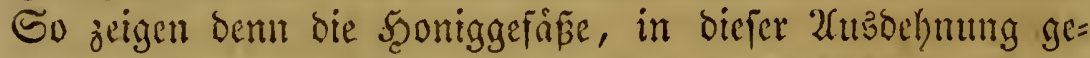
nomnten, febr viele Serichiedentyeiten in Geftalt, Lage und

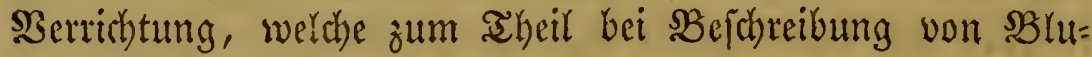
men, bie folde Srgane befiten, hervorgehoben werben follew.

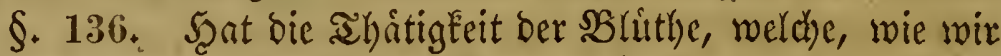

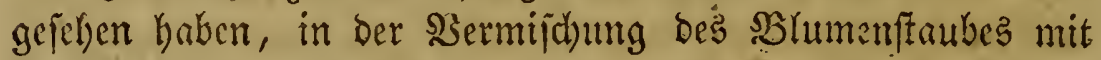
Der Narbe ifhr Siel findet, diejes erreicht, fo ift bie Bghume num mustos, ihre alyeile fallen baher ab, und nur der Frud)t= Enoten bleibt itbrig. 2(us biefem entwidelt fich bie Frutht (fructus). Nan findet an ihr bie vier alyeile bes Frudht: Envtens wieber, bod) alfe mehr altsgebildet uno zum Theil

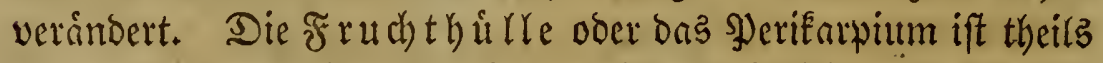
blattartig, theils holzig, theits fleifhig, theils aus ciner fleifdigen und ciner holzigen Schicht gebildet, und,

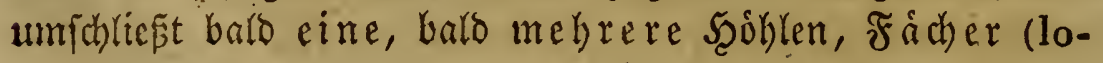
cula) genamt. Sind metrere Scoblen. ba, fo merden biejel= ben burd) S d) eidewa a nd e (dissepimenta) getrennt, und man erfennt gewóknlich f(t)on auf ber șberfliche ber Fruct)t bie

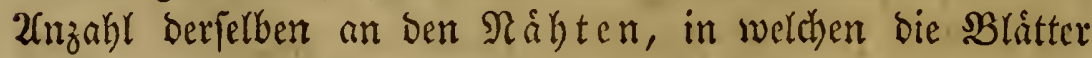
ber Fruchthillle mit cinander verwachjen find. Feglen bie Scheibewánde, was bisweilen ber Fall ift, fo filsen bie Mut= terfuthen an ben Ráften (placenta peripherica s. parietalis, 
wandfanoig) find fie vorbanoen, fo befinden fich bie Mut= terfuchen theils an ber Echeibemaino (pl. lateralis, feiten= ff ånoig), theils im Nittelpunft, wo fich bie Sdyeiberwanoe treffen (pl. centralis, mittelftindig).

$W_{3}$ as bie Form ber Frucht betrifft, fo hat man folgenbe zu unterfcheioen:

I. (Finfache $\mathfrak{F} \mathfrak{r}$ ich te (fructus simplices). Die Fruch)tbutule befteht nur aus einem $\mathfrak{B l a t t .}$

1. Se ich $f \mathfrak{r}$ ud $t$ (caryopsis), eine einfamige fructht, bei

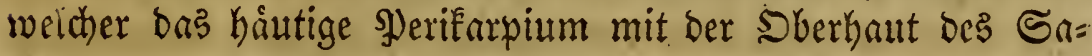
mens vermadjen ift. $-2 . \mathfrak{R} \mathfrak{u} \tilde{B}$ che $\mathfrak{n}$ (acheniurn), cine ein= famige Frucht, beren pergamentartige Şưlle eng um ben Sa men liegt, aber noch nicht mit feiner Sberhaut verwachien ift. - 3. $\mathfrak{R} \mathfrak{u}$ (nux), eine einfamige frucht mit holzigem Perifiarpium, bie unten in eine Şưlle, bem $\mathfrak{R}$ åpfchen (cupula), ftedft. - 4. Steinfrucht (drupa), eime einfamige

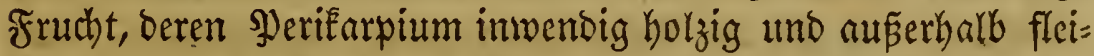
fojig ift. - 5. $\mathfrak{B a l g}$ (folliculus), eine mehriamige Frudt)t,

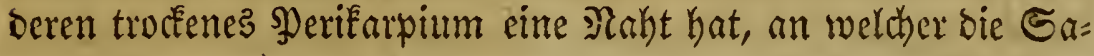
men fitsen. - 6. Scillie (legumen) untericheidet fid num burch bie 2(nwefentheit einer zweiten Rath, aber bie Samen fiken alle an ciner. - 7. SBeere (bacca), eine ein = ober mehrfamige Frncht, beren Merifarpium fleifhig ift uno bie Samen ganz einjohlieft.

II. 3 ufanimengefefiste Frud te (fructus multiplices). Die Frutchthillle beftegt nus mebreren Şláttern uno entl)ailt mel)rere Fádber.

8. Sd) o o te (siliqua), eine Yanggeftrectete trodfente Frud

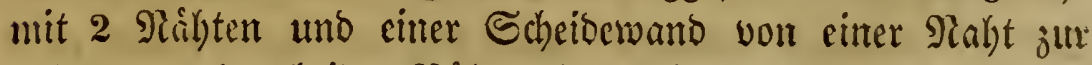
anbern, an ben beiben Răhten fitzen bie Samen. - 9. $\Omega_{1} n=$

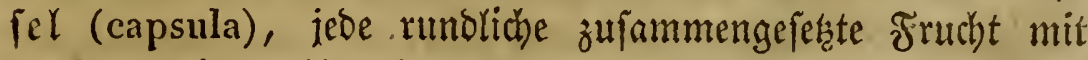
trockter Şutlle; bismeilen fehlen bie Scheibewainde, und bie Sapjet ift bann einfâcherig (unilocularis), gewobntich) mefrfád)erig (multilocularis), - 10. Stange (aurantium), hat cime leberartige. Şuille, und cin faftiges 3ellgewebe

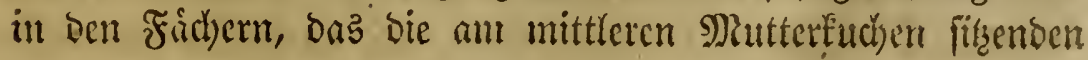




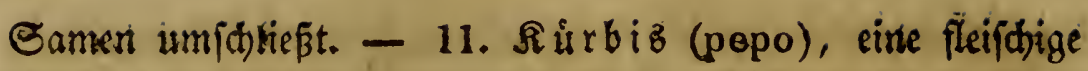

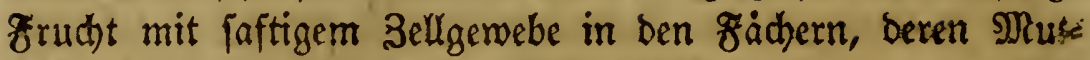
terfuthen am Umfange liegt. - 12. $\mathfrak{A} p \mathfrak{f e l}$ (pomum), eire

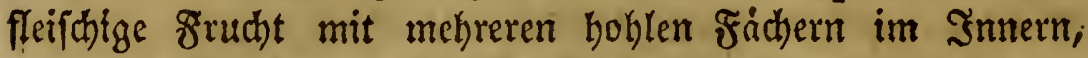
worin bie Samen. - 13. Steinbeere (nucularium), cine mehriamige Steinfrucht, bie theits freie, theits aus mefreren fulammerigerwach fent Steinchen enthailt. -

\$. 137. Unter allen Theilen ber Frucht ift Der Same (semen) Der vorzüglidffe uno widtigfte: (Fx entfteht aus Dem (Ei d) en burd) eine mannigfache Seitse von (Entwidéturi= gen, Deren einzelne Stufen, wegen Der Rleinheit Deș Begen= ftandez, fdimer begriffen merben, baher wir biefen fo in: tereffanten aheil hier unberúdfichtigt laffen múffen. SBismeis len ftedt Det Same innerbalb ber Fruththullle noch in einer feinen, neţartigen Şaut, bie vom Nabelftrange außgeht, bent Mantel (arillus). $\mathfrak{X m}$ reifen Samen untericheibet man

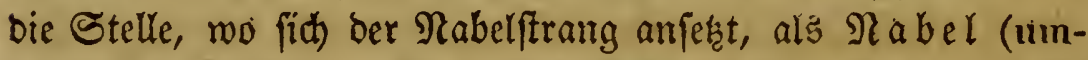
bilicus s. hilum). Thbeile beb Eamenz bemertt man vier: 1) Die áuß̈ere Bebectung bis Samenz, fie biloet eine biffe, meiffens gefärbte Şrut, Şdale (testa) genannt. Unter biejer liegt bei fehr viclen Samen 2) ein fleifhiger, mchliget ober yornartiger Rörper, bas (5imei B (albumen), weldhes ben Scauptnahrunģ Stoff ber Samen enthält, und immer, felbft bei ben

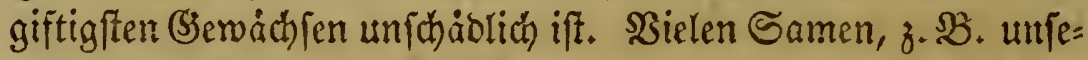

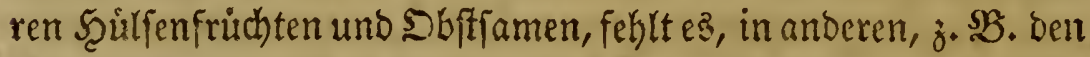

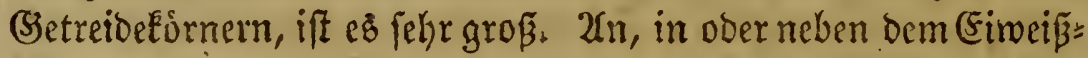
forper, ober wo biefer fel)lt, unmittelbar unter ber Schale, liegt; 3) Der męift grope, fleifhige S a ne enlap pen (cotyledon), mit wet=

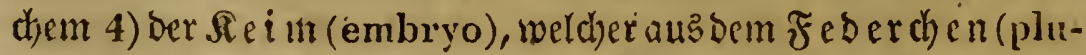
mula)umb dems is r zel d) en(radicula) befteht, zujammenlyingt.

Der Samenlappen ift balo einfach (Monocotyledones), barb boppert (Dicotyledones), und ericheint in er= fteren Falle als eine, im lebteren als z wei meiftens halb: fugelformige ober flache, rundiche ober geftrectite Sdhuppen, unter ber, ober zwifden weldyen, die a geile bes Seimes hiegen. Sie bilden zufinmen einen oben flachen, nach unten 


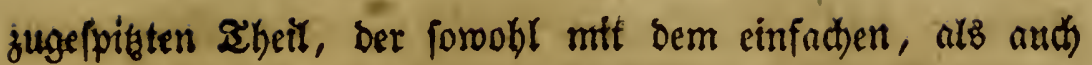
ben boppelten Samenlappen in Berbirioung fteht. Der obere, flachere, bismeilen am Ranbe gelerbte aheil ift bas geber.

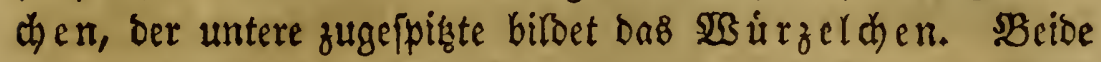
werben immer von bem Samenlappen verbedt, und bicfer liegt entweber unmittelbar unter ber Samenl)aut, oder neben

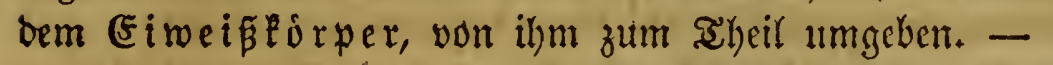

§. 138. Bjelangt mum cin foldes Samentorn in ben

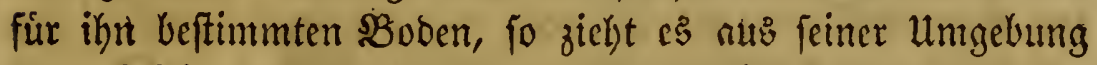
Feuthtigfeit an fich, und behnt fich vernittelft derferben aus,

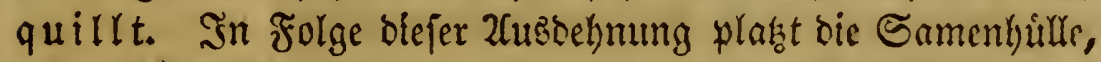

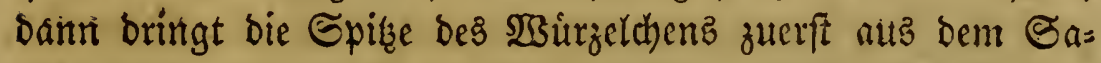
men bervor, uno fteigt num, Der Same mag aufrecht ober verfebrt in ber Eroe liegen, jebesmal fentrecht nach unten binab. Sein entregengefestez Ende, bas Feberchen, fudtht bent entgegengefesten $\mathfrak{S B}_{\mathrm{B}} \mathrm{g}$, unto erhebt fich thails altein, theils mit ben Samenlappen jugleich, Liber bie Sberfliche. Şier int sidjte entfalten fich bann bie Samenlappen, bas Feberchen wákhft zwijhen innen bervor, entfaltet fich-ebenfallz, bildet

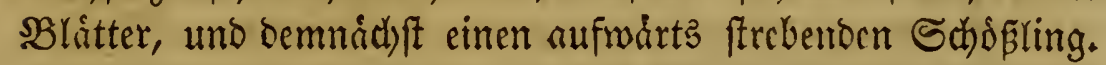
Sobald bie erften SBlitter gebildet fitto, vertrofinen bie nach und nach blattartig gewordenen Samenlappen. Diefer ganje Scergang heişt Reinung (germinatio).

Da alle \$flamgen Samen oder fantenartige Rórper her= vorbringen, fo fonmen fick natirlich alle burch Samen fort=

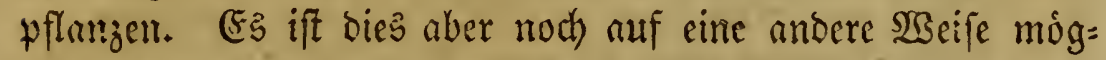
lidj, nelimlich durch Stecelinge. Schneibet man cinen jun=

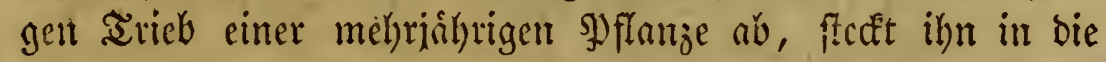

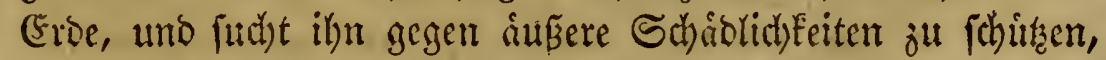

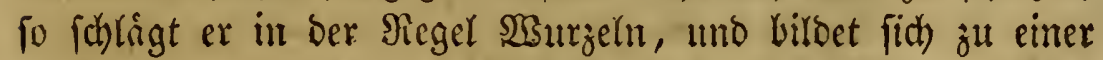

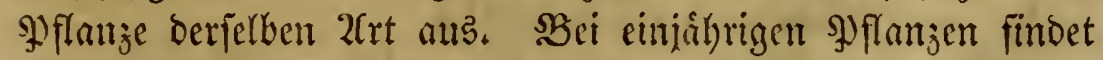

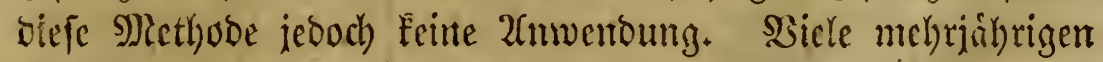

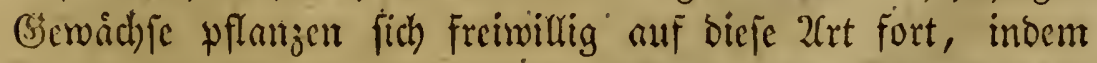

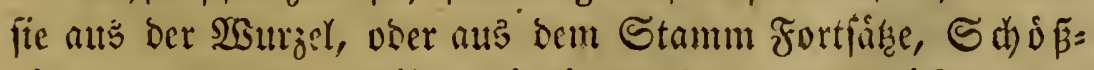

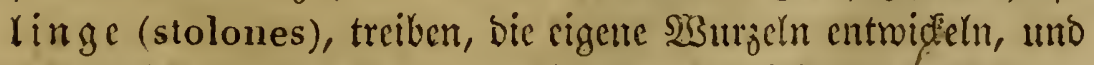

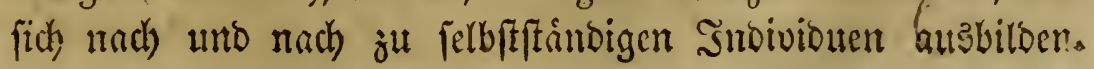


Scierourch erbailt man eine leichte Methobe, bie Baiume uno anberen Gicwaichje zu vervielfáltigen.

§. 139. WBie bie IIhiere, werben auch bic \$oflanjen nach ber Serifbiebenheit gewiffer Srgane in (jrupwen eirngetheilt, werche julimmen bas Sy)fem ber \$f flanjen bilden. Uun= ter allen (Fintheilungen ift feine, welche fo allgemeine 2tner= Eennumg gefunben hat, als bie bes fabmesiichen Naturforichers Linne, welcher bas Sylanjenreith nach den Serichiebentheiten ber Staubgefápe in 24 Silaffen, uno jebe nach ber sBitbung ber Stempel und einiger anberen Srgane in mehrere Soromun= gen eintheilte. Sein Syftem ift folgendes:

I. Pfanzen mit Deutlid fidtbaren Blumen. Phanerogamia. A. Staubgefáfie uno Etempel in einer Blume. Monoclinia, a. Etaubgefáfe mit Eeinem anderen Tgeile yerwadfest.

+ 2llle yon gleider \&ánge.

(Ein Єtuuggefáa.

1. Rlaffe Monandria.

3roci Staubgefáfe. • . . 2. - Diandria.

Drei -

3. - Triandria.

Sicr - $\div \quad \therefore \quad 4$. - Tetrandria.

Jün - . . 5. - Pentandria,

ভeç3 - . . . 6. - Hesandria.

巨ieben - $\quad . \quad$. 7. - Heptandria.

2(d)t $\mathrm{t}$ -.$\quad$ 8. - Octandria.

গeun - $\quad . \quad 9 . \quad$ - Enneandria.

$3 e \mathfrak{n}$ - $\quad . \quad$ 10. - Decandria.

3ebn bis zmanzig Etaubgefífe. 11. - Dodecandria. 3ranjig uno mejrere Etaubgefálse,

Die am Sield fif̧en. . 12. - Icosandria. 3wanzig uno mebrere Etaubgefáge auf Dem Jrumtboden fifend. 13. - Polyandria. tt $\mathfrak{B o n}$ ungleider \&ainge.

3wei lange uno jwei Eurze. - 14. - Didynamia. Sier Iange uno zwei Eurze. . 15. - Tetradynamia

b. Staubfáden unter irdy verwadfen. In einten ging. Sn smei Bimber. $\quad \cdot \quad \cdot 17$. - Diadelphia,

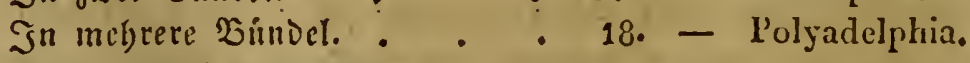

c. Etaubbeutel verwadícir.

Iltter fid in einen Riing. . - 19. - Syngenesia. Jist Dem! Etempel. . . . 20. - Gynandria. 
Erfte Rlcffe. Monandria, ạmeite Ilnf̣e. Diandria. 123

B. Staubgefápe und Gtempel in Deridie:

Denen Blumen. Diclinia.

Beide Blumen auf einem Stạmm. 21. - Monoccia.

Scioe Slumen auf verfóficı. Stámmen. 22, - Dioecia.

Dánnlide ooer rociblidhe ミlumen mit

"3ratterblimen auf Demielb. Ctamut. 23. - Polygamia.

11. Pflanzen obne fiftbare Blumen, : 24: - Cryptogamia:

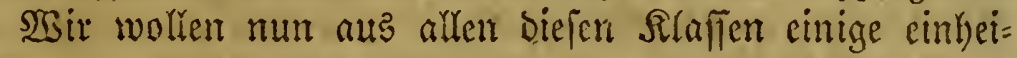
mijche, oder bejonders widhtige-sjflanjen ourdigeben.

\section{Erite Slaffe. Monandria.}

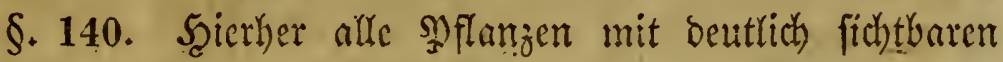
şlunten, in welchen fid) nur cin Etaubgefiśp vorfindet. Dicle Slaffe ift cine ber fleinften uno enthált meiftens aus:

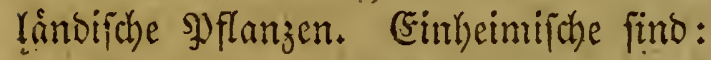

Der T a n n e n we bel (Hippuris vulgaris), ein Siraut, von 1' Lánge, mit grabe fébendem, faftigen Stengel, an weldem vicle Sireife quirlfórmig geffellter, linienfórmiger Slätter iber cinander. Die Elcinen Bhlumen ftegen ungeftielt in Den לlattadfich, baben Eeine Sirone, einen einbláttrigen Sield auf Dem Trudtennoten, an weidem

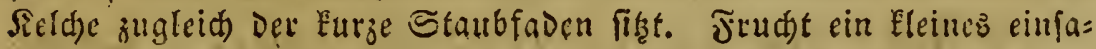

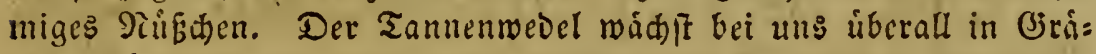

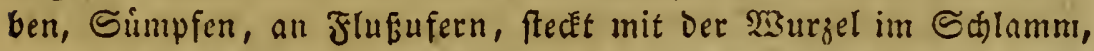

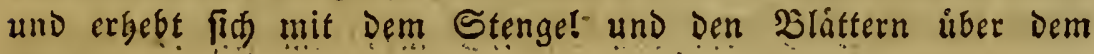
פ⿹勹巳

\section{3̧weite Slaffe. Diandria.}

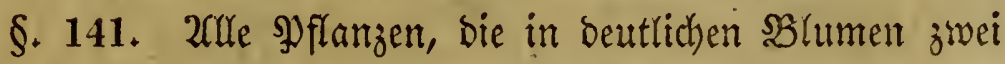
Staubfíben neben bem ein = ober mehrfachen Stempel zeigen, geboren hierher. "Die meiften haben nur einen Stempel, wo= nach \& inne bie Srbmung Monogynia bitbete, wenige befitsen jwei uno bilden bie jweite Soronumg Digynia, Die cinbei= miichen find alle monogynifh. '3. $\mathfrak{B}$.

Der SJartriegel (Ligustrum vulgare), eine ffraudjartige

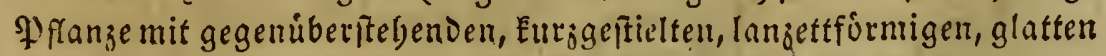
Slăttern uno traubenformigem Blütbenftande. Blumentrone eins blättrig, róbrig, Saum vierlappig, "meif́." Die Fruđtt eine zmei=

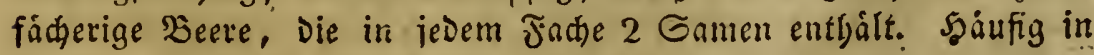
B̧ârten, fu Şecken und Rauben angepilangt.

Der \{panifde Fli eder. (Syringa vulgaris) bat die fient: 
getben ber vorigen Pflainge, aber Dle B!dter find breiter, Gerzfor: mig, und Dle Frudt ift cine zmeillappige, zweifdderige Rapfel, die aud) 2 Eamen in jevem zads entbalt. Bei uns in Şärten als Blerftraự, ftammt aus dem fróliden Europa.

Die Egenprets gattung (Veronica) bat simen 4-5=thoic Ilgen ungleldappigen field), eine tabformige ungleid)vierlappige

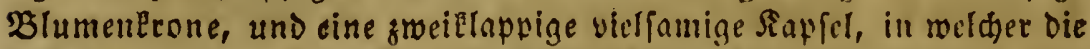
Sdeiderwánde an ben Slappen Rigen. Inter Den vielen 21 rten unfes rer B̈egend ift Die semeinfte V. chamaadrys; fte bat gegenuberftes Gende tiefgegagnte eiformize baarige Blatter, clnen adjelftsnbigen ágrenformigen Blúthenftand, vierlappigen Sield und eine grofie, blaue Blumentrone. Heberall auf J̈eldern, liegt all Boden, bod) Die Blumenifgre allitedt.

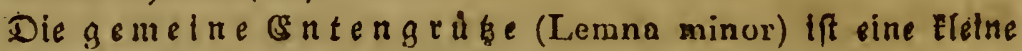
Pflange obne Stengel, mit linfenforunigen, giemlid Dicfen 3rattern,

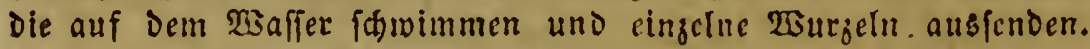
De Eleinen Blumen fiţen am Raude Der Blitter, Gaben cine ein= fade Blumengúlle und eine 1-2, fáderige Sapfer, worin einige Samen. Heberall gemein auf fegenden Beráfferk.

\section{Dritte Araffe. Triandria.}

§. 142. Drei freie, gleich lange Stanbfíden in beuttich fichtbaren 3witterbhumen bilden ben (E) aralter biefer Jinffe. Rach Der $\mathcal{Z n}_{j}$ ahl Der Griffel zerfallt fie it brei Sronungen.

\section{Dronung. Monogynia. Mit einem (5irifel.}

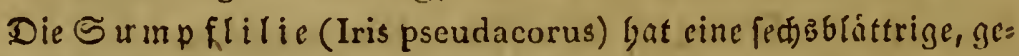
fárbte Blumenbillle, Deren Drei abred)felnde Bratter grofier find unD berabbángen, toabrend Die Drei Eleineren aufredte fteben. Der Briffel ift graar einfad, aber in brei blattartige Lappen gefpalten, Deren jeder cine Narbe trágt. Frudtenoten unter ocn Blumen, bilbet fid ou ci= ner Dreifäberigen, vielfamigen Siapfel aus. Die Pflanze bat fdmerdt: formige Blitter, welde Den aufredtftehenden, mebrblumigen Sd)aft am Şrunde fdoidenartig ungeben. Slumen forwefelgelb, fteden yor Dem 2lufblügen in ciuce Blumenfd)eise. Bemein in Sümpfen.

\section{Soronung. Digynia. Mit zoei Ssriffeln.}

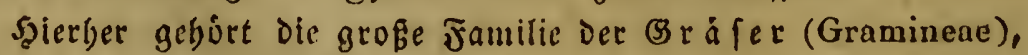

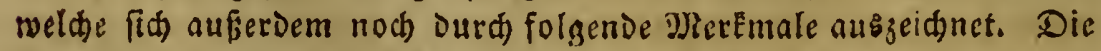

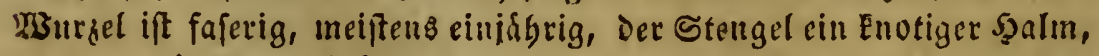
Die lisienförmigen $\mathfrak{b l a ́ t t e r ~ e n t f p i n g e n ~ y o n ~ D e n ~ s ̌ n o t e n ~ u n D ~ u m g e b e n ~}$ Den Şalm sine Strese fdeidenförmig. Biumen in sinfaden voer 


\section{Drtte Silaffe. Triandria. Bsraffer.}

zufammengefebten Xebren aud Rispen. Sede Blume bat eine zwels

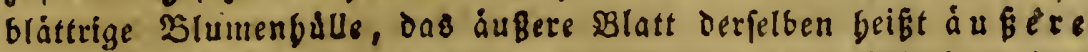
boer antere Spelic (palea s. valvula exterior s. inferior), Das iunere gegen die Xre des Zebre geridtete oft fcinere blatt wird in: aere oder obere Spelje (paleas. valvula interiors. superior) ge: מanut; blergu Commt bei rielen nod ein groltes, mebrere Blumen umfdlle Bendes, Blattpaar unter dem etfteren, weldes man nit Dem Namen $\mathfrak{B}$ alge (glumae) bezeldnet. Mad) Der $2 \ln$ jabl Der Blumen

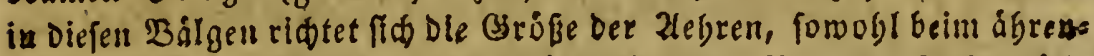

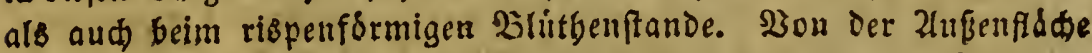
ber áuferen oder unteren Epelze entfpringt bel vielen Biráfru ein langer useiftens borftenformiger Fortfak, bie biranne (arista). -

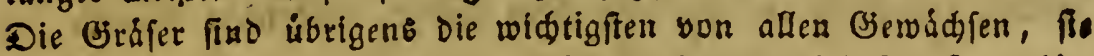
geben nidit blof dem Denfden felbft, fondern aud defien Şaustbies ren Die nothwendigften Nabrungomittel; Dafer ift ifre Jjenge to bedeutend, Dafifie Den zwandigften Fyell aller beEanten Pflanzen ausmad)en. Folgende arten fino befonvers widjtig.

Der Şafer (Avena sativa) bat einen gleiden rispenformigen Blitlyenftand; Bálge zmeiblumig, ungleid, Spelgen an Brunde báufig mit einem $\mathfrak{D a r t}$, bie innere uno obere an ber Spike gefpar, ten, die áfiete mit elner gedrebten Branne. Wird feit uralten 3ei= ten in Deutfiland angebaut, jegt aber meiftenz nur zu Blebfutter benubt.

Die Es er ft e (Hordeum vulgare), mit \&grenformigem Blitten: ftande, an jeder Stelle Der Zlabe dref einblumige Zebrden; $\mathfrak{B a ́ l g e}$ lang, Dinn, pfriemenfómig, untere Speize mit langer, fieifer, gra= Der Branne, Eamen von Saut ungeben, an beiDen Enden zuge: fpigzt, mit einer \&ángsfurde. Blumen in vier Reiben, ie zroci uno zmei einander gegeniber und abwedfelnd bober. Stammt aus Ei: cilien; fajon lange bei uns angebauet.

Der Roggen (Secale cereale). Dlútbenftand eine 2lebre; Bálge bunn, borftenspfriemenfsrmig, untere Epelze in eine lange, baarige Sranne verlángert, Die obere Der Bange nad gefaltet. Sas men glatt, cylindrifa, an beiben Enden abgeftukgt. Zlebrden frocis,

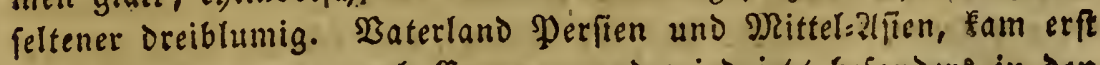
Durd Die 5y unnen nad) Enropa, und wird ieşt befonders in Den nisroliden Giegenden angebnuet.

Dic $\mathfrak{B}$ a izengattung (Triticum) ift febr reid an 2lrfen, alle

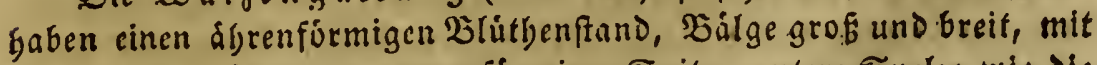
kủzerer oder língerer grannenformiger Spikge, untere Epelze wie bie Bálge, Die obete ziemlia Der Lánge nady gefaltet. Man bauet bei uns zmei Irten: Den Sommerwaizen (Tr. aestirum), mit vier: blumigen debren, Die Bálge glatt uno gegrannt; Den $\mathfrak{B}$ interwat: 
zen (Tr. hibernum), ebenfo, aber Die Sälge ofrie Granne. ftammen aus Dem Drient und wurbein fdon von Den älteften 2lcker: bau treibenden Silfern Eultivirt. Eine Dritte Ztrt, die $\mathfrak{S}$ ueE (Tr. repens), Gat vielólumize Alefrifen, Deren Bälge bals mit, bals olue Sranne; findet fid überall als İnerrut zwifhen Dem Getreide; und verbreitet fid febr fark wegen feiner Eriedienden Wurzeln.

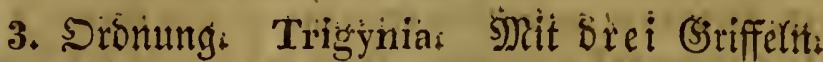

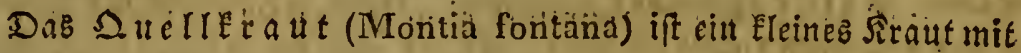
Eriecheridem Stengel, wornn aufrechtifebende 3meige mit gegeniber: ftebender, länglichen, ftumpfon Sláttern. Sllumen an Ende, funf: blóttrig, weiß: Jrudít eine siapfel mit orei Samen. 2(n feudteit

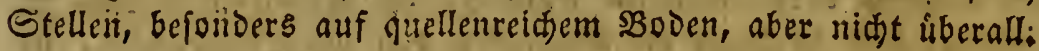

\section{Sierte Rraffe: Tetrandrid:}

§. 143. SSier freie, gleich lange Strubfáben bitben ben

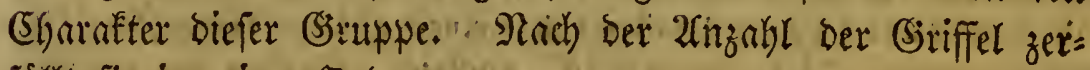
follt fie in vier Stomingen:

\section{Sromurig. Monogyna, Nit étrem Griffel:}

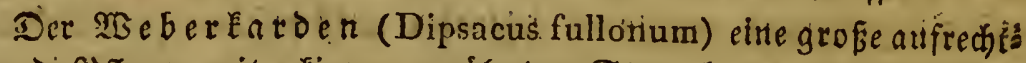
ftchende ?) Fanze mit ectigenr; geságntem Stengel und ungegeftielten;

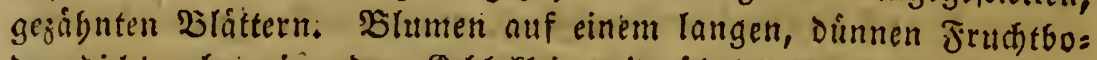

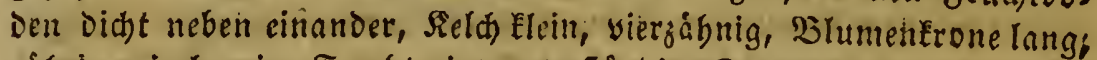
rógrig, vierlappig, Jrudte eine unterftantige Siaryopfiz: Unter jeder

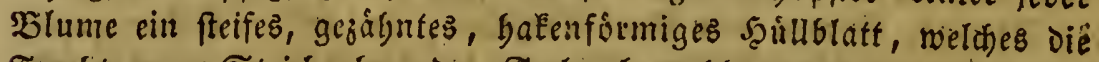
Frndit zum Stridggeben Der Tuche - Grandbar madtt. 3ü Diefend 3wecke bautet man die. Pflange in mandsen Bjègeriden. -

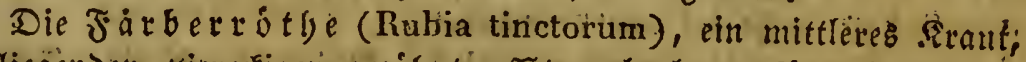
mit riegènoen, yiereséigen, gesábntén Stengeln; Ianzettfómmigen, quirt':

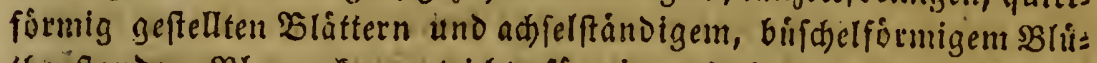
thenftanoc. SBlumenErone tridterformig, vierlappig, gelb; Jrudit zweifamig, Die Samen in ciner meideet Ştille. Dian genoinnt aus Diefer Spfange, befonders aus Der $\mathfrak{B}$ urzel, eine fajone, rotbe Jarbe;

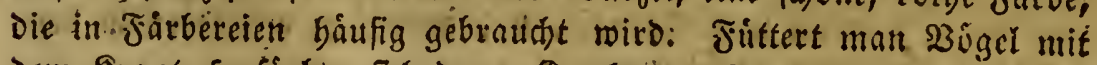
Dem Siraut, fo fârben fid Deren Sinodeti roth:

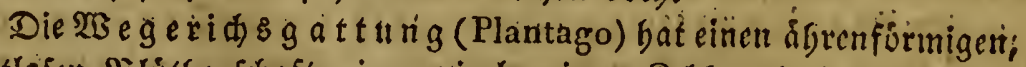

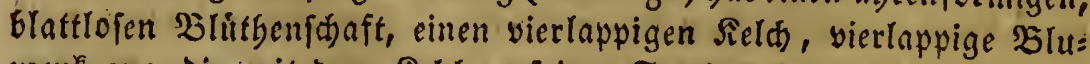

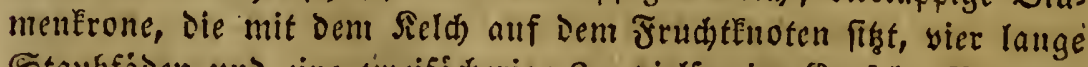
Etaubfäben uno cine bimeifád)erige 2-vielfaumige Siapfel. Dei unb, wadjen all 2Begen auf Jeldern u. Dgh., megreve 2trten, welde alle

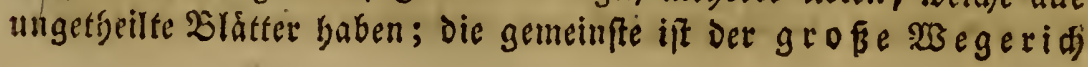


(Pl. major), mit grofien, ova!en, fumpfin, gratten, ranggefticten

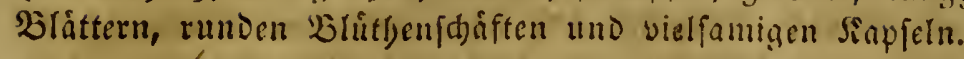

Die úbrigen orei Srommmen, mit zwei (Digynia), orei (Trigynia) und vier Briffeln (Tetragynia), enthalten feine wichtigen einkeimifhen (sinttungen.

\section{Fünfte Sifafle. Pentandria.}

§. 144. Sie ift cine ber joblreichfien, umb umfaf́t nlle Mflanjen mit fiunf gleich langen, freicn Strubfioben, bie mit bem Stempel in bericlben Shlume fitsen. Rinne theitte fie nach ber Salhl ber (Siriffel in 6 Sronungen.

1. Sromtng. Monogynia. Mit einem (5riffel. Scier= ber geboren mehrere grop̧e Familien einbeimifcher (semésthe unter welchen folgento bie wichtigften find.

1. Jam. Rălbbláttrige PF. (Asperifoliae). Eie baben eine énblättrige, rígrigs, fỉnflappige Blumenfrone, eine aus vicr einfantigen, rauben ooer'ftadeligen giufigen beftegende Frudgt, und abrvedjelnde raube Blátter. 3. $\mathfrak{S}$.

Das $2 \mathfrak{e r g i f p m e i n n i h t ~ ( M y o s o t i s ~ p a l u s t r i s ) , ~ e i n e ~ E l e i n e ' ~}$ \$fanze mit Tanjettformigen, ziemlid glatten Blâttern uno binmel= blauer SlumenErone, Deven gelbe Niunoung von Eleinen rundfiden

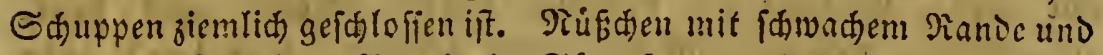
Trabel am Brunte. Semein in Eimpfen, am Rande yon Briben: *Der gemeine Steinfame (Lithospermum arrense) bat cine offene, freie Mhindung Der weifen, tridterformigen, ShlumenErone, und nicht geralldete, eiffirmige, nidft mit einem Tiabet ver jegene

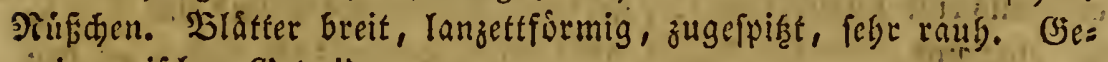
niein zmifden Betreibe.

2. Fam. Sd líffelblumenpflanzen (Primulaceăe)。 Sie. Gaben eine rögren = ober rabformige ShumenErone mit freier Mhins Dung. Blátter an Der Wurtel oder gegeniberftegeno. Joruht eine einfäherige, zwei = bis füfelappige Siapfel.

Die gemeine Sdifuffelblume (Primula veris) bat rifg= renformige, bingende, gelbe $\mathfrak{B l u m e n}$ am Ende cines blattlofen blis

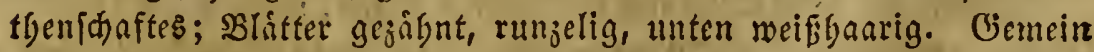

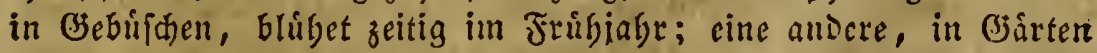

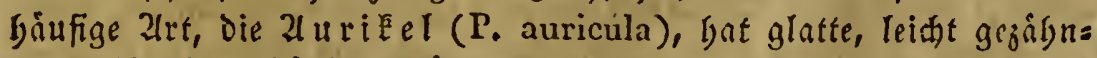
te, meglartig beftåubte Siátter, und Dunfelrotgbraune Şlumen mit getber MRübung. Stammt aus Stalien. 
Der Fif ber I lee (Menyanthes trifoliata) bat ju brei geftellte, langgeftielte, eiruube Bldttben und einon anfredson Slithenfdaft; 3lumenfrone weib, tridterfirmig, tieflappig, inmendig raug; Rap: fel onetElappig mit malloftándigen Muttertuden. Bei uns Gáafig auf feudten Wiefen und in Gुrdben; wird gegen das Ealte Fieber angemendet.

3. Zam. Rartoffelpflanzen (Solaneae). BlumenErone trióferfórmig, unterftóndig, Blatter abmedfelno; 2 গarben am Birifiel. Frubt eine 2-4=fádjerige, eben fo viel Elappige Rapfel oder Beere, mit yerdidtem Mutterkuden an Den Sheiderodnden, Biele Pfangen Diefer Jamilie fino giftig.

Die $f_{\text {a }}$ to ff el (Solanum tuberosum) Gat eine glodenformige, gefaltete SBlumentrone, fegr Eurzgeftielte, langlide, fpiz̧e Staubbeutel und eitue jweifáderige, vielfamige \$Beere. Blätter gefiedert, baarig.

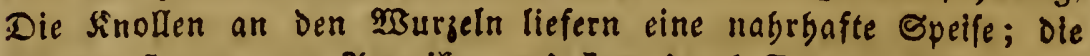
Pranze frammt aus 2lmerika, uno Eam Durd frang Drate in Der erften Şälfte Dez fechszegntẹn Jabrbunderts nach Europa.

Die Tolleirfde (Atropa belladonna) gat eine glocenfor: mige, braunrotge Blumeatrone, uno cine beerenartige, fdwarge

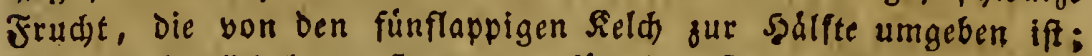
Blátter ziemlid langgeftrecte, Gergformig. Sm mittleren und fúb; liden Europa, befonbers in Bsebirgsgegenden am Rande yon bैebu: fden; fegr giftig uno gefágrtid.

Der $S t e$ a p fel (Datura stramonium) bat aud eine lange, trid)terformige, gefaltete, welfe Slumentrone, aber bie Frudt iff eine grope, vierfäderige, ftadelige Siapfel. Dildter groß, breit, augebuthtet, wellenformig ain Dande gebogen. Das 1-2' gobe

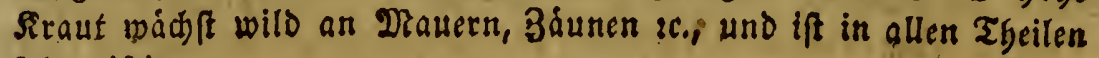
fegr giftig.

4. Fain. Dindenpflanzen (Conrolvulaceae). Sie Gaben ebenfalls eine tridterformige, gefaltete Blumenerone und eine $2-4$ : Elappige und faderige Sapfel, aber bie Sdefdemánde ftofien an ben Rand De: Slappen uno die Samen fiken am Brunde ber Shet= Dewánde."

Die 3 a unwinoe (Convolvulus sepium) bat eine grofie, weißge, trichterfórmige slumentrone; Gerzformige, abwechfelnde Blátter, und eine Dreifádherige Siapfel, jebes Jach mit 2 Saunen. Ğemein in Gibuifden.

Dit in Die erfte Dronung Der finften Silaffe, wenn gleid zu fei:

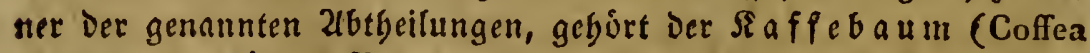
arabica), ein mäbiger $\mathfrak{B a u m}$ mit gegenúberftebenden, eiforntigen, ziem= lịđ fpişen Bláttern uno quirlformig geftellten Blumen in Den slattadfeln. Reld fünflappig. Blumenteone cinblattrig, in 5 tiefe 


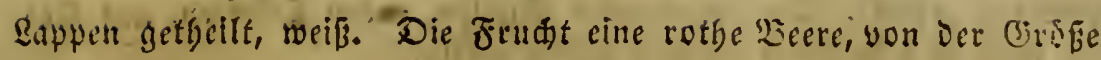
einer Şafelnuff, Darin jwei grofe Samenkorner, welde die befann=

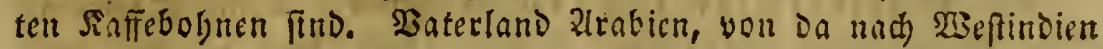
verpflangt.

\section{Stonitug. Digynia. Mit z}

In biefe Zbtgeilung gegort Die grofe Familie Der Doroens pilla nzen (Umbelliferae), welde diefen Tamen yon ifren Bli= thenftande beEommen babcr. Es find faft alles Erautartige ein:

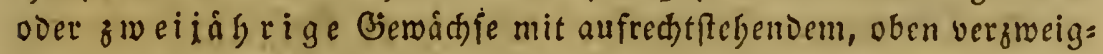
tel:t, oft boblcm, Enotigem und aukerbalb gefurdtem Etengel; melre mals gefieverfen Blattern und Eleinen in Dolden ftegenden 3 la: men. Jeoe Bilume gat 5 fidmale Seldbrafter a:if bem Jruditfno= ten uno 5 oft ber for ormige Blumenblátter. Sie Frudt beftegt ans zroei yon Didéer Szaut ungebenen, oft mit Mippen, Stadeln uno Jlïgeln ver: febenen Samen, die im unreifen 3üfande felt aneinander bángen,

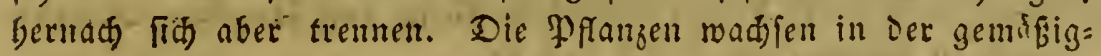

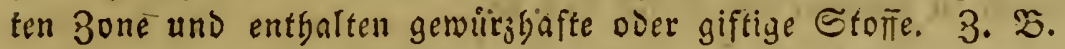

Die Diogrtíb (Daucus carotta), nit fiederipaltigen, zer:

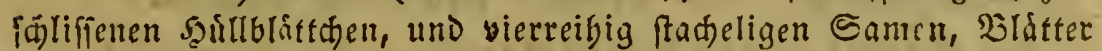
und Stiel fteifbaarig. Tiegen Der bisken, orangefarbigen, fleifdigen,

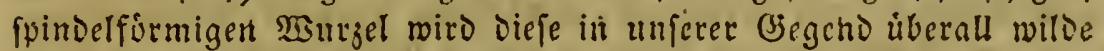
J) flange in Gaárten ferge allgemeint argebautt.

Der $\mathcal{A}$ il m mel (Carum carvi) bat einèn cinblittrigen, allge:

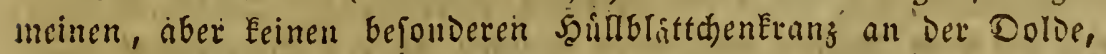
und lisiglidfrunve, faft fỉnfeckige, mit 5 ftumpfen gippen verfegene Eamen. Blitter Dreimal gefiedert, blittchen fatmal linienformig.

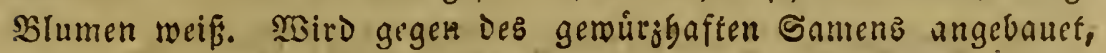
twádit aber auds willo bei uns.

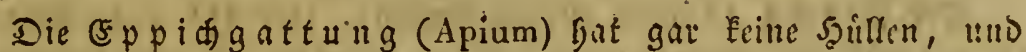

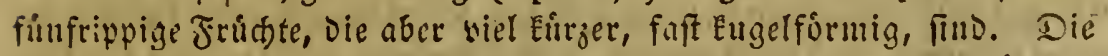
Deferfilie (A. petrosclinum), mit winkeligen Etingel, glínğen= Den Dráttern; Die unteren Bláttfien Drcithcilig, Die oberen lansetts fórmig, gánjrandig. Der Sellerie (A. graveolens), mit gefurd)= ter! Stengel, uno gefieberten, Eeilformigen, eingeithnittenen Etengel:

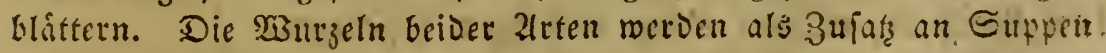
gett)an, uno aud fonft gegefien; bei jener ?trt ift fie tang, fpiner!s firmig, bei Diefer differ, melge Enollig, mit vielen zhusftrablungen.

Der'gefleck te S Gierling (Conium maculatum) Gat brive Salfen, Die Jrúnte eifórmig, mit 5 ftumpfen Dippen, bie bei Des

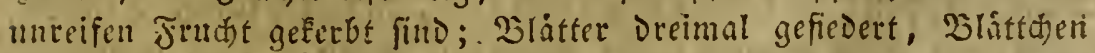
Elciu, glánjeno, lánglideciruno, tief gegálgnt; Stengel glatt, DunEel

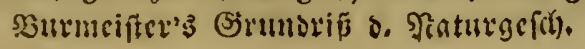


blutroty geflect. Zn angebauten Ctellen, neben Dorfern, auf 50. fen u. f. m.; in allen Tgeilen bodsft giftig.

Der $\mathfrak{B}$ afferfdierling (Cicuta virosa). Seine allgemeine Sallle, Die befondere vorganden. Frudte rund, breit gedrudt, mit breiten Pippen und Dazmifden bervorragenden Furden. Blatter zmeimal gefiedert, Slätthen lang, lanjettformig, gejafnt. WBurzel grof, Dick, freifhig, inwendig bogl, aber ourd Sheidemánde in $\mathfrak{F} a$ : đer getbeit (radix loculosa). Wád)ft in Eráben uad auf Jlof. bolf; ift giftig, aber weniger als Der yorige.

\section{Sronung. Trigynia. Mit brei Biriffeln.}

Der gemetne Soll un o e oder F Tieder (Sambucus nigra) ift ein Strauch mit bolgigen 3meigen, in welden ein febr meides, fđmammiges Nare. Die Bläter unpaarig gefiedert, Blátţen oval. lanzettformig, geságnt; Sluthenftand eine Zfterbolde; Field füfs zábnig; Slumentrene einbláttrig, in fünf Lappen getbeilt, weißs;

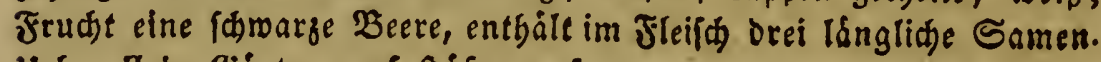
Heberall in B̈ärten, auf Şơfen u. f. w.

\section{Dronung. Tetragynia. Mit vier Briffeln.}

Die Sumpf = Darna Eleine Prange, mit aufreci)tem, winfeligem Etengei, berzformigen Slättern, fünfbláttrigem Reld), 5 weifen Blumenblättern, eben to vielen fouppenformigen, am Rande nit geftielten Sinjpfen befegten Sonigorifen, uno elner einfád)erigen, vierElappigen Sapfel, in wele

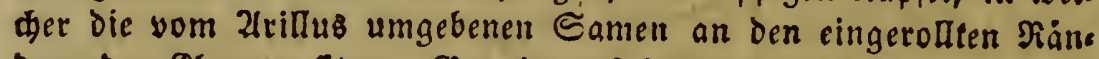
Dern Der Silappen fį̈en. Bemein auf feudten Wiefen.

\section{Sronung. Pentagynia. Mit fünf Griffeln.}

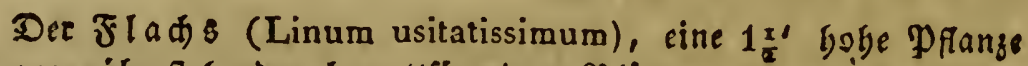
mit gegenüberftegenden Tanzettförmigen Brittern, b̆lauen fünfblätts rigen $\mathfrak{J} / u m e n$, und einer runDen, zebnfäderigen, zebrfamigen Siapfer.

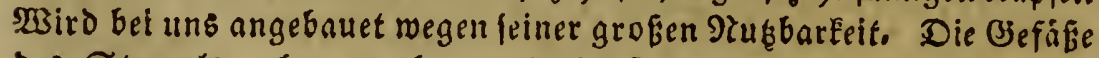
Des Stengels geten, nach manderlei Bubereitungen, Den Fla ds.

Die Yeste, fech)fe soromung, Polygynia, mit fehr vies

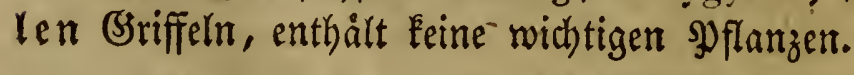

\section{Sedifte Sifafe. Hexandria.}

§. 145. Şierber gehören faft lauter \$flanzen mit einem Samenlappen; fie haben alle fechs freie, ziemlich lange Staub= fáden mit bem Stempek in Derjelben Strone, und meifens 
cinen sinfad)en (stiffel; boch fommen auch $2,3,4$ und 5 Griffel, felbft fehr viele, vor.

\section{Srommg. Monogynia. Mit einem Briffel.}

Dabin vicle 3riebeipflanzen. 3. $\mathfrak{B}$.

Die weife $\mathfrak{a}$ itie (Lilium candidum), mit aufredfffegendem,

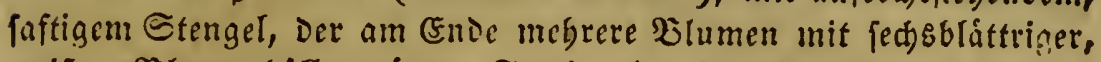

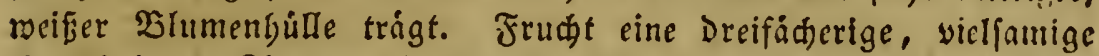
Siapfel, Deren Silappen Die Edeidemánde in Der Mitte tragen. Sas men am mittleren MutterEuden in Doppelter Reibe. Sei uts in Bârten, frammt aus Silcin = 2hiten, 2(trmenien 2 .

Dic gemeine 3 wiebel (Allium Cepa) Gat einen goblen, une ten baudjig ermeiterten, blattlofen Etengel, Der an feinem Ende viele in einen runden Snopf geftelte Blumen trágt, Die yor Dem 2lufblüben in einer trodenen Blütbenfdheide eingefdloffen fino. Jrudt Der yorigen Pflanze, aber Eleiner. Sủd. Europa, mirb bei uns angebauet. Bistreilen bilden fid oben grotlden Den Blumen junge 3 miebeln.

Der $S p a r g e l$ (Asparagus officinalis), ein ftaudenartiges Siraut, mik aufredbffebendent, áftigem Etengel, und vielen feinjertbeilten,

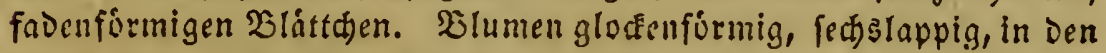
Blattadfelin. Jrudte elue rothe, Dreifaderige beere, jedes Jad mit 2 Samen. Die jungen Eđüflinge Der alten $\mathfrak{B u r z e l n}$ liefern ein fegr woblidmeçendes, nabrbaftes Giemúfe, weabalb man diefe Pflan z\& bei uns allgennein in S゙ärten fultivirt.

Die Sayasint be (Hyacinthus orientalis) Gat einen aufredten, blattlofen blúthenfdaft, traubigen Blütbenftano, glo cécufórmige, feçelappige, gefárbte $\mathfrak{b l u m e n , ~ u n o ~ e i n e ~ b r e i f a ́ d j e r i g e , ~ v i e l f a m i g e ~}$

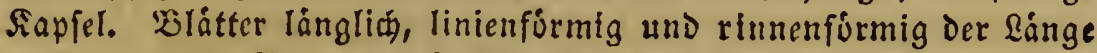
nad) vertieft. Dei uns als 3lerpflanze in 3immern, ftamm aus Dem. Drient.

Die $\mathfrak{T}$ u Ipe (Tulipa gesneriana) bat einen einblumigen, blatt: lofen Etengel; fedbsblättrige, gefárbte Wlumenbúlle; breifádterige, vielfamige Sapfel, und eine Dreiectige Narbe bie unmittelbar auf

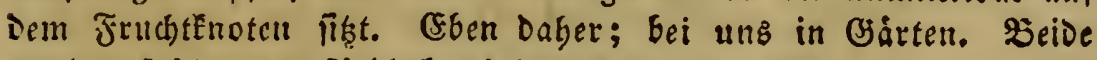
wourden frúber aus Riebgaberei fegr tge:ter begablt.

\section{Sromung. Digynia. Mit zwei (3riffeln.}

Der it e if (Oryza sativa), ein Gobes 'Bras, mit Didfem, fleis fđigem Şalm, uno grofen, brciten Bláttern. Blútbenftand rispen= fórmig, Baflge cinblumig, Epergen am Brunde fdjmielig, zufammen= georidft, lederartig, aum Eamen zum Theil fefegenadfifen. Etammit eigentlid aus Dftindien, wo er in feudter Eumpfgegenden mild 


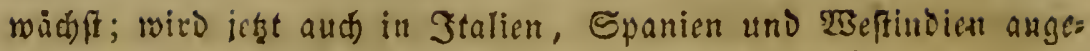
bante. Geine Eamen liefern ein legr beliebteb ङ̈emúfe.

\section{Dromung. Trigynia, Mit brei Giriffeln.}

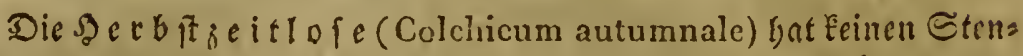
gel, fondern sie Dlume und Die breiten fđeidenformigen Blatter erbc= bet fid unmittelbar aแs Der SBurzel; Diefe eine 3wisbel. Blumen= frone fleifdfarben, lang, tridhterformig, oben fchslappig; Fruát ci= ne Drcificherige Rapiel, icDes Jach mit wielen Gamen obne Sronung am Jumentumel. Eie erfdeint erft in folgenden Frubjabr liber ier Erde, wabrend die Slmme im Spátberbit bervorbribt, Daber filius ante patrem. 2luf $2 \mathfrak{3 i e f e n ~ u n d ~ i n ~ B a r t e n ; ~ c t r a s ~ g i f t i g . ~}$

Die ubrigen Sronungen enthalten Feine widttigen SDfianzen.

Siebente Siafle. Heptandria.

§. 146. Dicfe (3ruppe, weldye burch fieben freie, gleich) lange Staubgefípe, bie mit bem Stempel auf bem: felben Frucistboden ftehen, charafterifirt ift, enthislt nur me= nige Splanzen; z. $\mathfrak{S B}$.

Die Rof Eaftallie (Aesculus hippocastanum), ein Gober Baum mit weit verbreiteten 3 weigen, moran fiebenfathe Blatter und traubenförmig geftellte blittgen. Sede Dlume bat einen ginblattri=

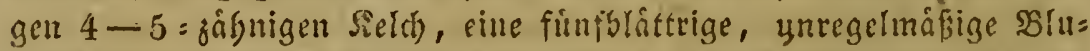
menerone, gebogene Staubfáden, und eine grofe, Dreifád)erige und DreiElappişe, oberfándige Siapfel, an Deren Silappen Die Encrides

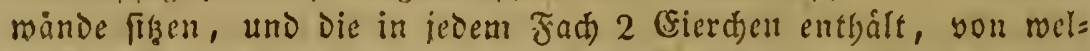
then 6 aber in Der Megel nur 2 over 3 jur Entmidelung Fonmen.

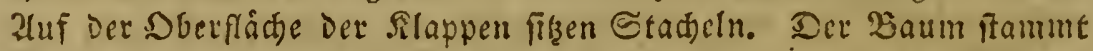
aus oem Drient, und wird bei ans bur 3ierde befonders auf Spa=

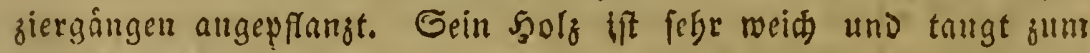
Sauen nidt.

\section{Xd de te alfife. Octandria.}

§. 147. 2xd)t freie, gleith lange, mit ben Stempel in oerfelben \$Blume befindiche Strubfíben bilden bas Sennjei= den biefer Srruppe. Sie beftebt aub vier Sromungen.

\section{Sronung. INonogynia. Mit cinem Miftid.}

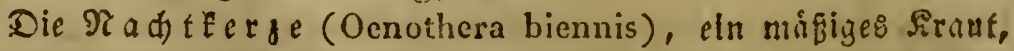
mit grabeftefendem Stengel, worall oval: lanzettformige, gum Theil berablaufende $\mathfrak{E l a d t t e r , ~ D i e ~ c t w a s ~ h a n t g ~ f i n d . ~ D i e ~ g r o f i e n , ~ g e l b e n , ~}$ 
Dlerblattrigen biumen fteben in ben Blattadffetn, uno geben eine vierElappige, vierfádberige Sapfel, mit viclen baurlofen Eamen am mittleren DiutterËuden. Stuf trodnen, fonnigen Jeloern.

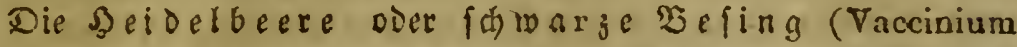
myrtillus), bat einen 1' Golen verzmeigten Stengel, mlt ETeinen, ei=

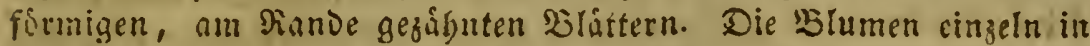
Den Wlattachjeln, mit fleifhfarbiger, einblättriger, blafiger Blumen= Frone auf beml Frudtenoren, einem vierzábnigen Relch, - uno einer 4-5. fücherigen 'Bsere, Deren viełe Eamen am Snnenubinel iedeß

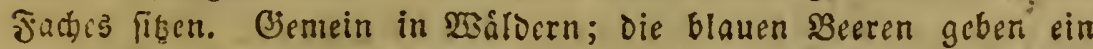
fïmacibaftes Piabrungsmittel.

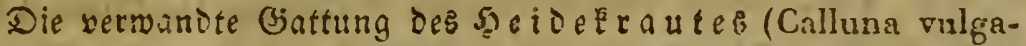
ris) bat Elcine, fohuppenformige Brátter, und rótglidfe, cinbláttrige

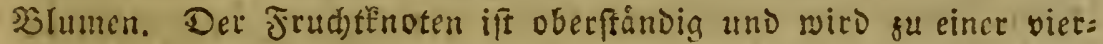

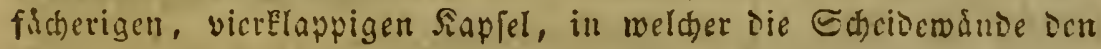

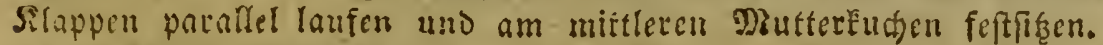
Gemein alf allen Syeiden.

Die 2. Sronung Digynia enthalt keine widstigen (S) nttungen.

3. Srontng. Trigynia. Mit brei Etempelt.

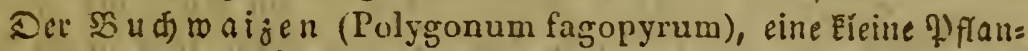

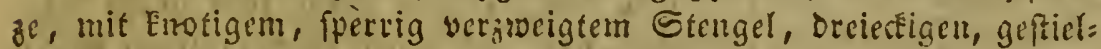

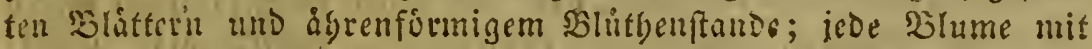

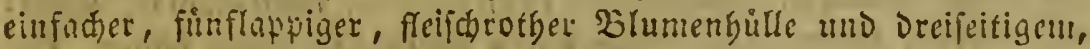
fónarzem, glingendem Eamen, wether yom perigonium eingefúllt

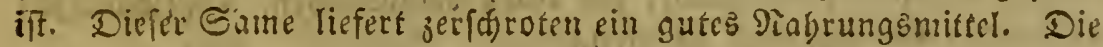

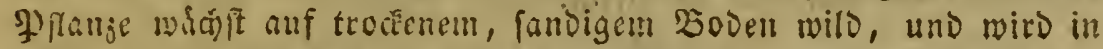
mandjen Gigenden aligebautet.

4. Stonung: Tetragynia. Mit vier SJiffillen.

Die Einbeere (Paris quadrifolia) bat einen graden, ungetţeil=

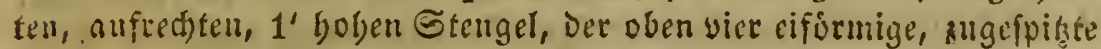
Blåtter trigt; in Der Ditte berferben entfpringt ein DItumenftiel,

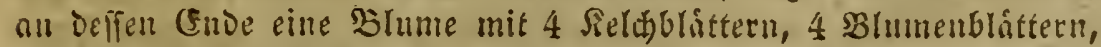
8 blatturtigen Staubfíden, und einem runden vberftindigen Frutt: Envfen, welder fich ju einer vierfáderigen, vielfamigen, fidwar

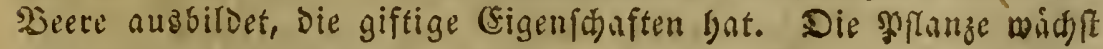
nidft felten in feudten Bebifficen.

Reunte Slaffe. Enneandria.

§. 148. Mit neun freten, gleith) Kangen Staubfiben neben

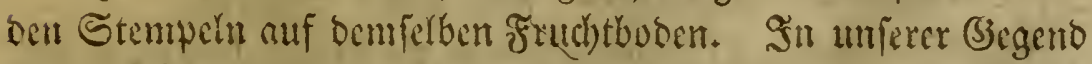

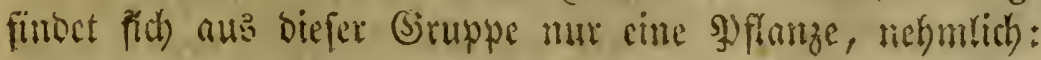


Der $\mathfrak{B}$ affer liefd) ober Die Doldenbinfe (Butomus umbellatus), mit bobem, grave auffeigendem, zelligem, faftigem, unser: zmeiglem Dlithenfdaft, Der an feinem Ende viele boldenförmig ge: fitlle Slumen trảgt, und am Girunde yon menigen, fónalen, zuge= fpiketen Blattern umgeben ift. Jebe blume mit fedsblätriger, fleifd: rotber Slumengille, 9 Etaubfáden und 6 Stempeln, Deren jeder fich ju einer einfädgerigen, yielfanigen, längliden balgkapfel entwidfelt. Zn ufern von Seen, Teiden, Fiuffen und in Oráben nidjt felten.

\section{Selinte Sifafle. Decandria.}

§. 149. (Fine etwas gróbere (siruppe, bie burch zebn freie, glech lange, neben bem Etentpel, theils auf bem Frudts boden, theils an oer \$Btumenfrone, befeftigte Staubgefápé be= ftimmt iit. Sie bat 5 Scauptoromungen.

1. Sronung. Monogynia. Mit einem \$ुiftill.

Der $S_{u}$ ufros marin (Ledum palustre), cin mápiges Siraut, mit boljigen, jertbeiften હtengeln, woran abwedfelnde, berftreute, linicnfírmige, oben DunEelgrine, glánjende, unten braune şrátter, und bifhelfórmig geftellte, meifie Solumen an Ende Der 3roeige.

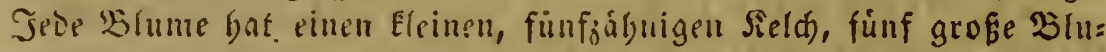
menblátfer, uno einell cinfaden, oben mit einem fünffrabligen Stern

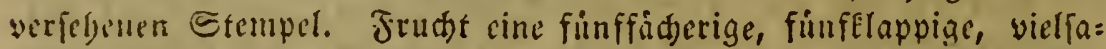
mige Siafiel, Deren Silappen fid unten neben Dem Etiel offinen. Die Pfinuze widgft al: Zorfmoor, bat einen betáubenden, Siopfidmergent crregenben ઉerud, uno ift gifftig.

\section{Sromung. Digynia. Mit zwei (3riffern.}

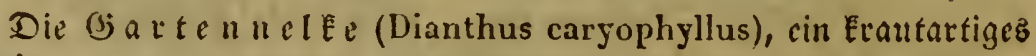

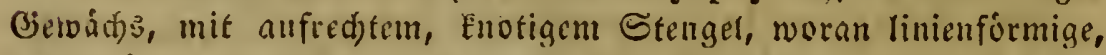
riunenfirmig verticfte, gegeniberftebende, meiggrime, beftáubte blät= ter, uno gipfelftánsige bilumen. Der Seldh ift rófrenformig uno bat an feimem Grunde mefrere fouppenformige Blátter; Die fínf Błu: menblitter find unten fegr fidmal, erweitern fid aber nadj oben in einen Dreifeitigen, ain Paande grzifnten, Eappen. Sie Jrudbt ift eine einfächerige, vielfamige Sapfer, Deren Samen am mittlersn Wruttr:

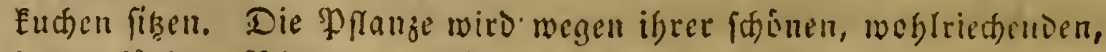
bantgefóróten $\mathfrak{Z}$ lumen in Gárten gejogen, uno ftanme eigentlid) aus Staliell, wo fie mild wadift.

3. Sromung, Trigynia. Mit brei (3riffeln.

Der Ster nu miet (Stellaria alsine), ein Eleincs, faftiges Siraut, mit nieverliegenden 3 meigen uno gegenúberfténenden, oyalen, juge: 
fpişzen Bláttern. Die Eleinen, weiffen Blumen ftegen am Ende Der 3weige, baben fủnf Seldbblatter, und fünf tiefgetgeilte, meife Diu: menblätter. Frud)t eine vielfamige Siapfer, Die mit fechs Silappen auffpringt uno Die Gamen am mittleren Mutterkuden trảgt. Lueber: all gemein, auf Şófen, all $23 e g e n$, felbft auf Der Strafer. Die fris fhen Blätter frelfien Die Sanarienbigel gern.

4. Sron. Tetragynia. (Entí)ålt Éeine michtigen \$g flanzen.

5. Soron. Pentagynia. Mit fủnf (̧riffeln.

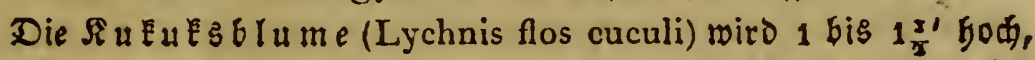
bat einen grade ftebenden Stengel, mit gegenúberftebenden, 低mulen Blättern und mefreren blutrotben Blumen am Ende. Jede Blume beftelyt aus einem baudigen Feldh und fúnf $\mathfrak{B l u m e n b l a ̈ t t e r n , ~ b e r e n ~}$ jeves un grei fdmate Gauptlappen gefpalten ift, welde an igrer $\mathfrak{B e r}$. einigungşfelle nod) einen fomalen, Bunzeren \&appen ( $\Re$ agef, unguis) an jeder Seite neben fï baben. Jrudbt eine einfáderige, vieraamige Siapjel, Die mit 5 Zágnen auffpringt. Heberall gemein auf W̉iefen.

Der Mauerpfeffer (Sedum acre), ein Eleines Siraut, mit Didén fleifdigen Bláttern und gelben Blumen am Ende ber 3meige.

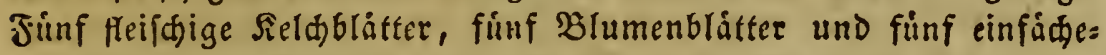
rige, vielfamige $\mathfrak{S}_{a p f e l n}$, bie am ङ゙runde unter fid zufammengángen. Bentein an 216 Gängen, neben Dem 23 ege, an Dúrren, fandigen uno founigen Stellen.

\section{Elfte Rlaffe. Dodecandyia.}

§. 150. Sn biefe (Sruwpe fteltte $\mathbb{L}$ inne alle গyflanzen, bie mit bent Stempel auf Demelelben Fruthtboden mehr als zely $\mathfrak{n}$, aber wentiger als $z$ wan $z^{i g}$, Staubfáden haben. Şier= her melyrere SDronumgen, $\mathfrak{z}$. $\mathfrak{B}$.

\section{Dromung. Monogynia. Mit einem \$iftill.}

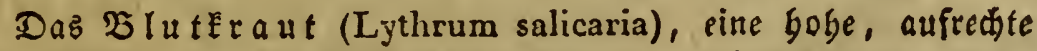
厄umpfpranze, mit Gerzformig:Ianzettliden, gegenúberftegenden Blät: tern und äbrenformigen Blíthenftande. Sede Slume gat einen eins

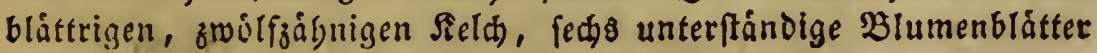

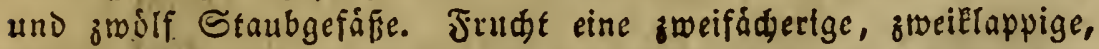

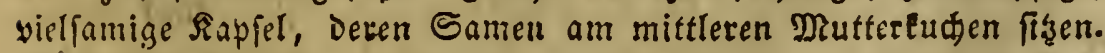

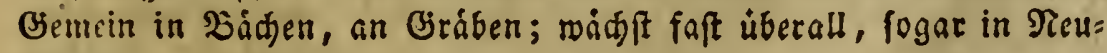
Syolland.

\section{Sromung. Digynia. Mit zwci \$ुiftillen.}

Der Doermenni (A (Agrimonia eupatoria) wdd ft an fọnnigent

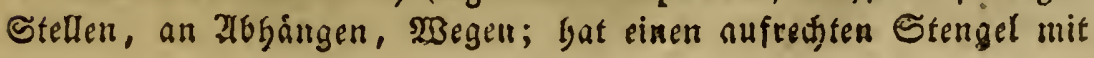


ábrenfómig gepellten Brunen uno geficderten 2jláttern; jebes Blättáen eiformig und gebínt. Die Blume bat einen untirfản: Digen, mit bakenformigen borften beferzten, fünflappigen sield, fünf uid)t grobe, gelbc Blumenblätter, dis auf Dem Sicld fiben. Die Frudit Geftebt aus zowei Samen, Die von Dem barten, faheligen Sield eingefidolofien fino.

\section{Sronung. Trigynia. SMit brei \$liftillen.}

Div $\mathfrak{B}$ olf $\mathrm{z} m$ il $d$ (Euphorbia cyparissias), cin Eleines, aufrect)= tos, $\frac{1}{2}$ bobes, faftiges Staut, mit vielen fdmalen, linear slanjettfor: zuigen Blittern, aus welthen, wie auth aus Den Stingeln, bei Der. Der= Ictzung eine weifse, fáarfe, etwas giftige Milch bervorqullt. Blu: men ant Ende, liber bieiten, abgerundeten Şúllblattern. Jede Blume bat einen geftielten Jiudfboden, an Deffeim Piande 4-5 halbmonofors

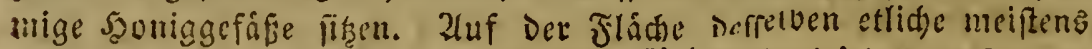

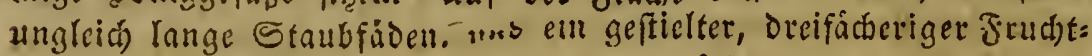
Fnnron, wetder fith zu einet grofen, oreifád)ertgen, in jedem Jach cin fdwarzes SamenEorn entbaltenden Siapfer ausbildet. Iuf fonni: gen Jelocrn, Niefweiden, an WBegen ac. Febr gemein.

Die Ba te n = Refed a (Reseda odorata), ein einjábriges Siraut, mit ziemlitb langen, lanzettformigen, gegen das Ende etras breiteren Blattern, und ábrenformig geftellten, moblried)enden 3lu= men. Der Seld ift megrlappig, Die solumenblatter find in viele fäderformige Rappen getbeilt, und Die Jitudt ift eine cinfodycrige, unregelmáfige, am Ende offene Sinplel, Deren Gamen an manoftún: pigen Jitterkuthen físen.

\section{3 wolfte Siraffe. I cosandiria.}

§. 151. In biefer (Jiruppe ift nicht melyr bie 3ahl ber. Staubfiben, fonbern bie Stelhung Derfelben Das Scauptmere: makl. Die hierher gehorigen \$flanzen zeigen nehmlidy mehr als zwanzig greich lange, freie Staubfáben, bie auf'bem Seldi) befeftigt find. (Ebenda fiten auch bic Bzlumenblitter; Die Etempel aber ruhen auf Dem Fruchtboden.

1. Sromung. Monogynia. Mit einem Stempel.

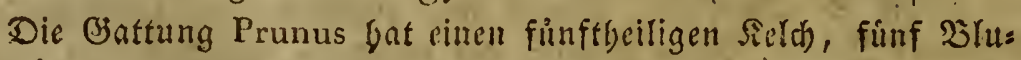

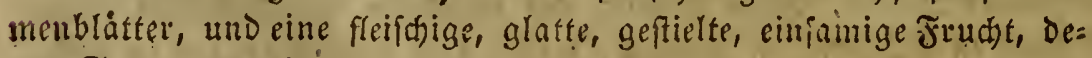
ren Same yon einer bor jigen glatten Sthare umgeben ift. Dabin

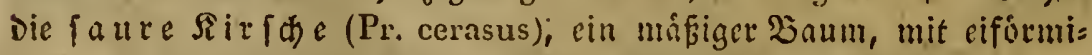

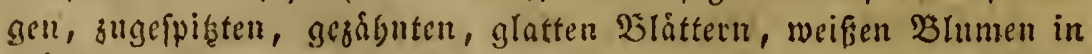
Buifcheln, uno rotgbraunen, fauren Frintgen. Stanumt aus Silein: 


\section{3̧oófte Sinffe. Icosandria.}

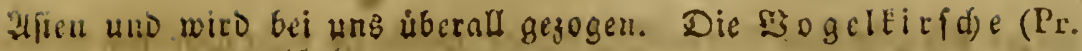

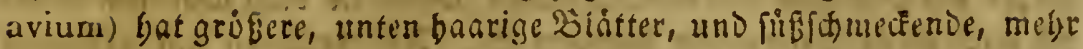

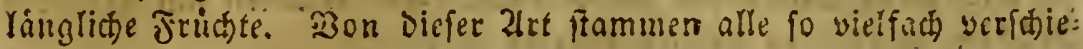

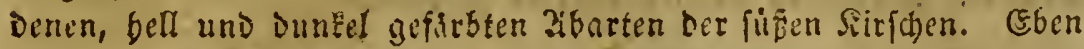

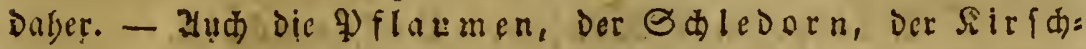
Iorbeer u. y. a. gegüren bieber. Rabe verwandet if Die Battung Amygdalus, wobin Der $\$$ fi $i$ fid $b$ a u m (A. persica); fie unterfici=

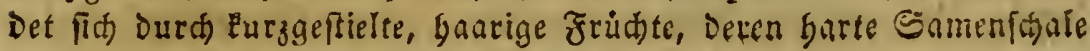
alif Der sufferen Dobefláche febr runzelig. iff.

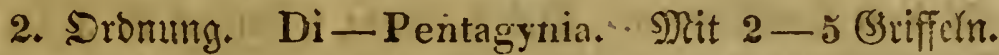

Dic Bantung Pyrus gat 5 Briffel, 5 fidldlappen, 5 Brannen=

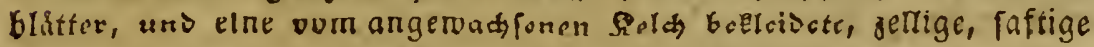

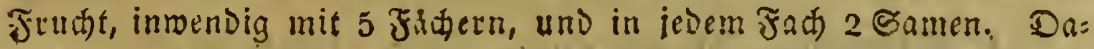
bin: Der 2 p felba u u (1. malus), ein bober $\mathfrak{B a u m}$ mit unterbalb baarigen כlattern und runden Fridgten; und Der $\mathfrak{Z}$ irnbaum (P. communis), mit glatten Blattern und Eolbigen Frúdten. Beibe ftanmen aus Stalien, Briect)entand, Silein, 2(fien, und baben fít Durd die Siultur bu ben mannidjfadffen Spielarten yerindert.

\section{Sronung. Polygynia. Mit vielen Etempeln.}

Die ßo fengattung (Rosa) bat einen oberftandigen, fünftheia figen Sield, Defien Eappen ficderipaltig getheilt fino, 5 Blumenblit: ter und fére viele Staubfiden. Die Frud)t (Syainbutte oder

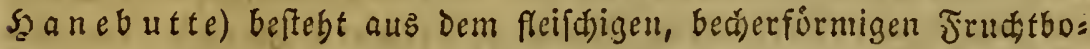

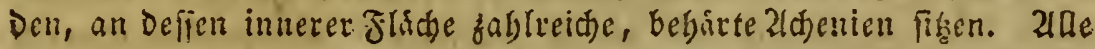

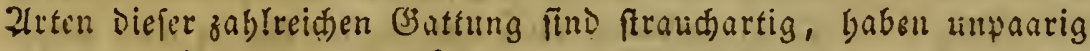

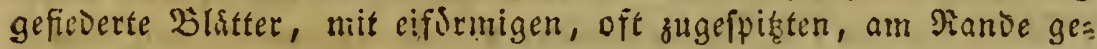
\{ágten blättchent. Jian zieft fle wegen ibrer fajongefírbten, wobl=

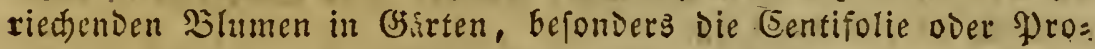

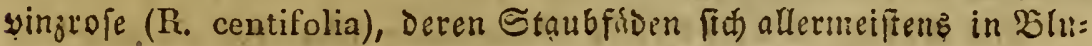
menblifter vermandeln, Dafer fie fegr frarle gefirlt iff.

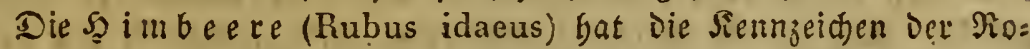
fen, aber Der Frudtefnoten ftert tiber Dem Reld und biliet fich auf Dem kegelförmigen FrudtboDen zu vielen, Eleinen, einfamigen, cine

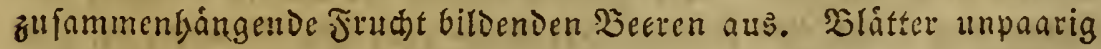

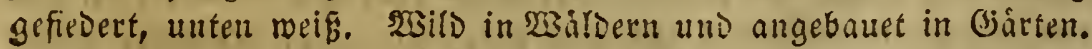

Die $\mathfrak{W}_{3}$ alderd beere (Fragaria vesca) ift nur Erautartig, bat

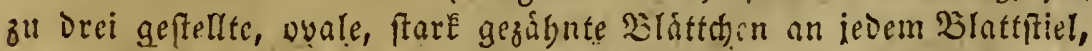
einen buifchelfórmigen Bhîtgenftand, einen Doppelten zegntheiligen

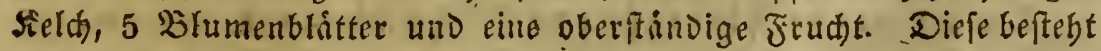
aus Dent weiden, faftigen, Didéch, Engelformigen Frudteboden, Der

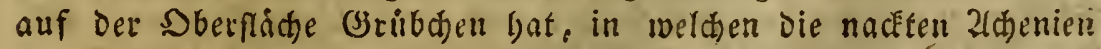
liegen. Die \$fange findet fid überall wild in YBaldern und yer: 


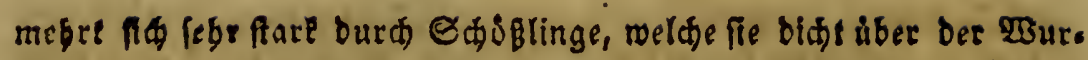
fel nach allen Eeiten bin bervortreibt. 2ndere 2rten mit gróferen Jridten werden in unferen Biarten gezogen.

$$
\text { Sreizelnte Rlaffe. Polyandria. }
$$

§. 1522. Die Senmzeichen diefer Siruppe ftimmen mit benen ber vorbergebenden iberein, aber bie vielen freien Strubfaben fiken nicht am Reld), fondern, wie die Stempel, auf Dem Frudytboden. BBei manchen sjflanzen ift Seld) und Blumenfrone nicht beutlich gefdeden; oft find beide gefärbt, ober beibe gruin.

\section{Sronung, Monogynia, Mit einem (Siriffel.}

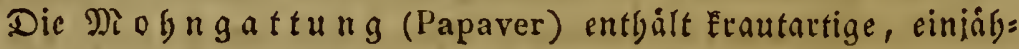

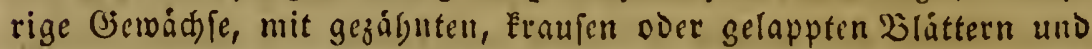
verzweigtem; aufrectem Brithenftengel. Seve Blume bat einen z we iblattrigen feld, Der nad Dem Zufbliben abfält, vier grofé Zlumenblátter, und als Frudt eine grofe, faft Eugelrunde Rapfer, Die von Der fternformigen Rarbe gek̂nt ift. Die Rutterkuchen bilden breite Reifen inmendig am Ranie Der Sapfel, und tragen fehr viele Gamen, weldye aus Eleinen Sodbern unter Der Sarbe her: ausfallen, wenn die Sapfcl reif ift. Bei uns Eommen drei 2lrten, alle mit Dunkel feutrotben $\mathfrak{B}$ lumen, befonders zrwifhen Dem G̈es treide vor. P. rhoeas mit madter fiapfel und abftebenden Szaaren Des Slumenftieles; ; P. dubium, aud mit nacketer fiapfel aber anges Drifften Scaaren Des 'Slumentieles; und P. argemone, mit lángli= der von fteifen Borften bekleideter אapfel. Der Saft yon P. somniferum ift Das $D p i u m$.

Die weife $\mathfrak{B}$ affer rofe (Nymphaea alba) Gat einen 4-5=

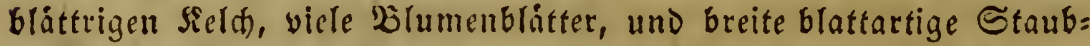
geffife. Die Frudt ift eine vielfdderige, faftige Saplel, mit ftern= formiger Parbe, uno enthät in jevem Fad viele Samen, Die yom Diantel umigeben find. Die Pflange waidf bei uns in Teidhen, am PRande yon Ecen uno Jlüfen, bat grofie elliptifhe Blätter, Die nuf

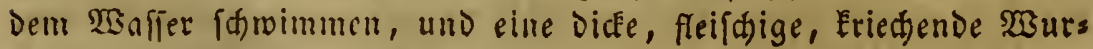
bel, Die im Edblamm fieclet.

2. Sromung. Trigynia. Mit orei Stempeln.

Der geme ine gi it terfporn (Delphinium consolida) radeft zwifhen (jerreive, mird Eaum 1' bod, bat iperrige 2lefte, jertbeilte Slåtter, mit linienformigen Eappen uno Junfelblaue Birumen. Die Blume bat eine gefärbte, fünfblattrige Szillle, Deren oberftes in einen langen, bohlen Sporn verlangert ift. Snnerbalb Der J̧ülle 
4 Sgonigorifen, ie zmei yon gleider Erobee, die oberen in cinem lan=

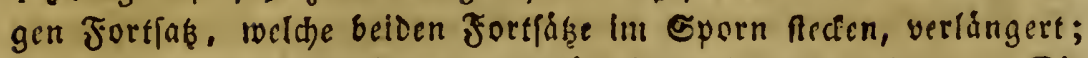
Die mebr unteren, feitfidjen gafenformis nad) yort gebogen. Die

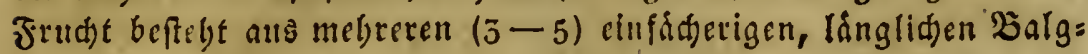
Eapjeln, Die fidh am Snuturande sfinneri.

3. Sromung. Pentagynia. Mit fiu $n f$ Stempeln.

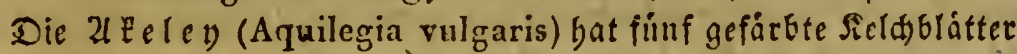

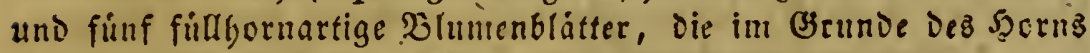
eine Syonigoruife tragen. Frud)t keftegt aus fünf Sialgeapfeln, die am Innenrande auffpringen. Die Plange if ein ausbauerndes 23alogemids mit zertbeilten, tunblappigen Blättern uno DunEels bTauen Blumen. Jaan giegt fie aud) in Barten.

4. Oronung. Polygynia. Mit vielen Stempeln.

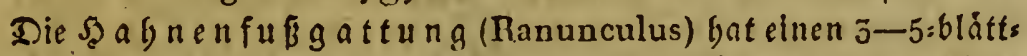
rigen Sicld, welder nad) bem Zlufbliben oft abfällt, und eine fünfo blättrige Sirone, Deren bidtter am Brunde eine fduppenformige

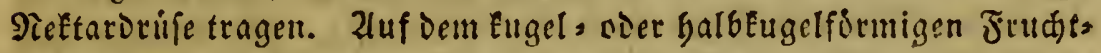

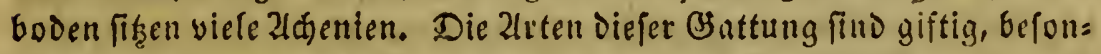

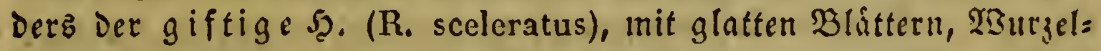
blätter Dreitbeilig geftielt, Die Eappen miever Dreilappig, abgerunvet, Etengelblátter in Drei fómale, Sinienformige Rappen gefpalten; Seldf

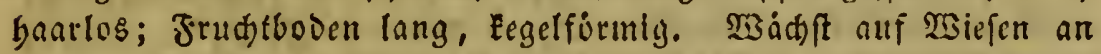
fendten Etellen, ill Galbtroçnen Bredben u. Dgl. giemlid) bäufig.

Die $f i$ u $b$ I u me (Caltha palustris) bat ganz Das 3 (nfehen eines gianunfulue, aber Der Seld und die Sicetardrífen am Brrunte Ber. SItumenbláter feblen. Frudt befitegt aus mebreren Balgeapfeln, Die fidi) all Der innern Tatl Sffnen. Blumen gelb, Blatter foft runo, ftumpf geságnt. Segr gemein auf allen risiefen.

Sierzehnte Slaffe. Didynamia.

§. 1ว3. Daz Renmzeid)en biejer Flaffe bildon bie zwei langen unb jwei furzen Staubfäben, weldhe mit ben Stem=

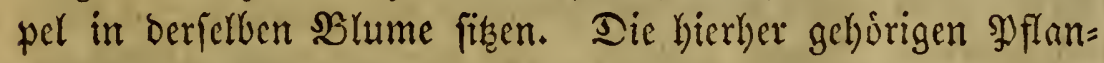

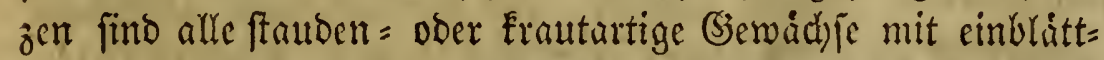
riger, unterftindoiger Błumenflone unb gegeniberffelyenden Sten=

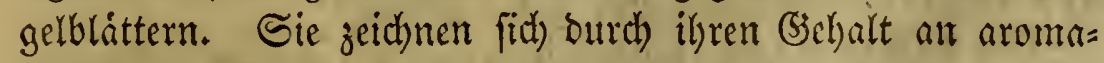
tifchen oder foharfen $2 \mathfrak{r}_{z}$ neiftoffen aus. Sinne theilte fie nact) Der Form ber Frucht in 2 Sronumgen.

1. Sronung. Gymnospermia. Die Frucht beffedt aus

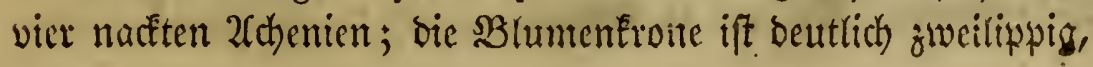


Der Siachen offen; Die Stengrlbtitter fieben gegenuber, uno Die Blumen quirlformig in ben SBlattachleln. Die Sुlfanzen billoen eine grope familie, welche ben ?amen sippenth men (Labiatae) erbalten bat. S. $\mathfrak{B}$.

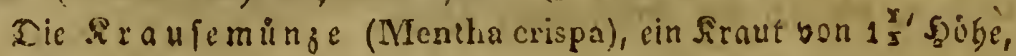
mit glänjenien, Gerzformigen, Eraujen und rauben 2ilattern, melde einen eigenthumlich riectenden Duft verbecten; Blumen Blatulich mit

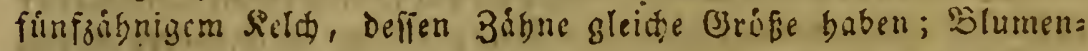
Erone sierlappig, der obere Rappen breiter, an Ende ausgerandet. Die Staubgefápe aufreht und auseinander itebend. Sn fíbliden Begenden, bei uns angebauet in B3irten. SWird zu Brifepulver und nagenftirtendem sbee benutgt.

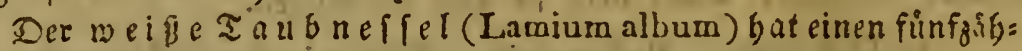
nigen Reld, Defien 3ibne in eine feine Borfte verlangert find; Die Blumentrone bat eine meite baudjige grundung, groci Sippen, yon welden Die untere breit, gefpalten uno jeberfeits mit zwei fleinen 3 ibuen verieben, Die obere belnartig gemolbt ift. Etaubbeutel in Der oberen Sippe; ORübden undeutlid Dreituntig. Die Blifter find

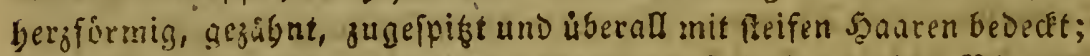

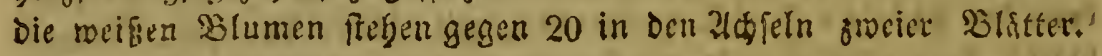
Semein an Bamen.

Der 2 cȳeran (Origanum majorana) bat eine fúnflappige Şu: mentrone, zivei etwas fobmäere Sappen biloen bie Ober:, Die anderen Drei Die Unterlippe. Die slumen ftegen in ie sier 2lebedien uno

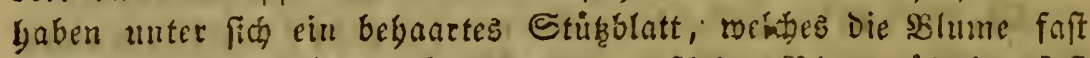
gang verocut. slatter eiformig, glatt, geftielt. Blumenågrshen faft Eugelformig. 2lus Palaftina, bei uns in Gärten, cinjäbrig. TBird.

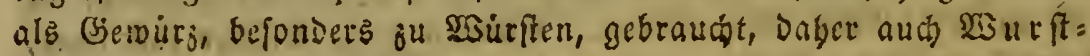
Éc a t.

2. Srommit. Angiospermia. Die Fucht ift cine jrei= facherige, vielinnige Raplet, in weldber bie Samen an ocm aus ber verdidten Scheidewano beftehenoen, mittleren snatter=

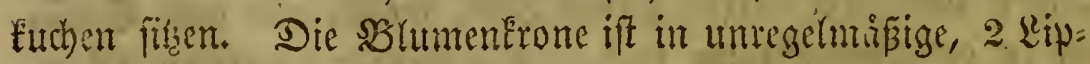
yen bilbende, anppen getbeilt, und bie Unterlippe verfechliefst

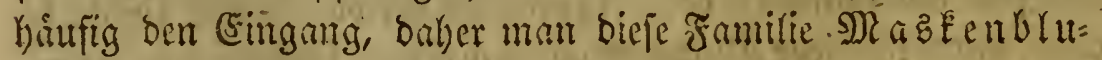
men (Personatac) genannt bat. Sabit:

- Dab \&owen ma l (Antirrhinum majus), eine bobe Stande mit lanzettformigen, gegeniberftebenocu glittern und dgrenformi=

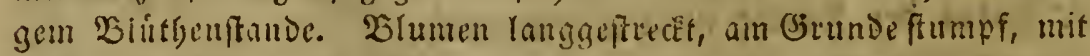
jewci Deutliden, gefdlofienen Sippen; reth, mit gelolidem Miunde.

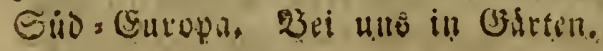




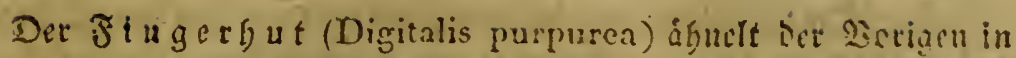
Form, Briffe und Brithenfand, aber sie lange am Brunde fpilse

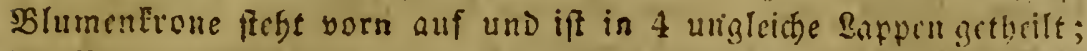
Der MitterEuden biloet Eeine Saciormand, fondern ift frei. Ditume

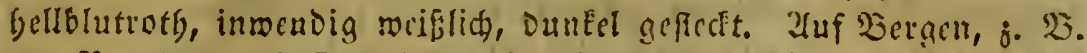
am Birocken uad) Slifuburg bin; foi uns in Badten. Biftig, als

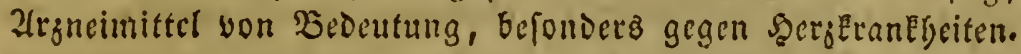

\section{Funfzélte Slaffe. Tetradynamia.}

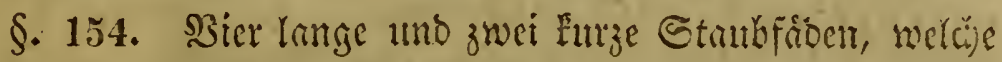
mit bem eimfachen Etempel fich in berperleon salume befinden, bitsen nach Linne ben (Sharafter bicjer Finfle. Sie hierber gehorigen \$fiamzen find durd)gehenos frautartige, meifens nut

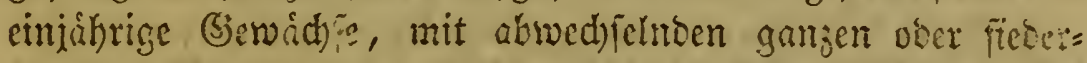

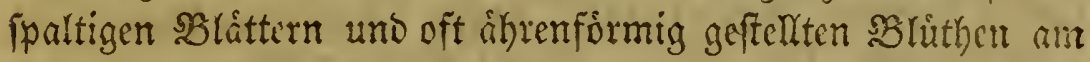
(5noe ber Tricke. Sebe shlume hat einen vierblattrigen Seldh) und vier freie Blumenblifter. Die Frudthte fint Schotent, bald langgeftrecte, vieljamige, kald furrjere, eiformige. Şier=

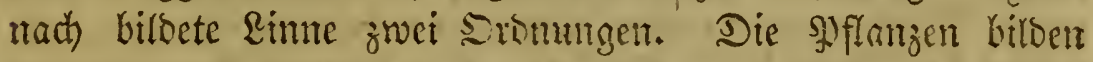
zugleich auch eine grope Familie, bie man wegen ber Ereuz=

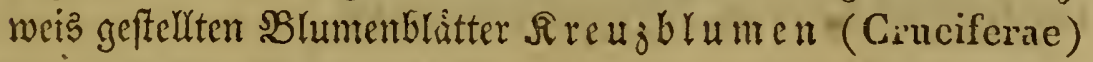
genannt yat.

1. Sronung. Siliculosa. Mit flciten, eifórmigen, mei= ftens menigamigen Edjotthen. Dabin:

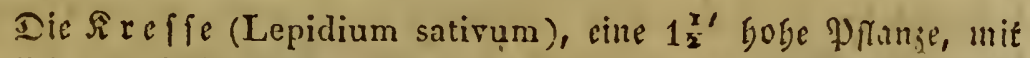
ringliden, vielfpaltigen Brlittern uno Eleinen, weifen BInmen.

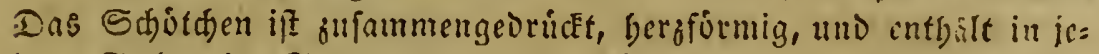

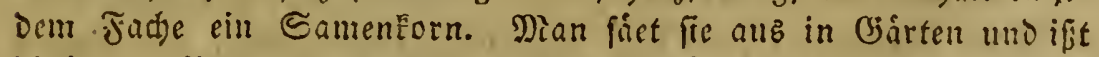

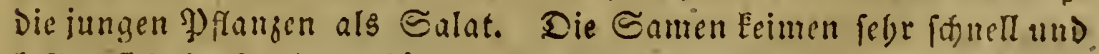
Iaffen fint in feudtem siffpapier, Das um Jlafden gewunden ift, zur Cntrwickelung Eringen.

Das Tafde ell $\mathfrak{E}$ a ut (Capsella bursa pastoris) bat cill Drci, ediges, hánglid) berfformiges, oben ticf ausgefdnnittenis Edjotdjen, Deffer Eafnformige STappen Ecinen Jlhigel bilden. Die Đflanje ift

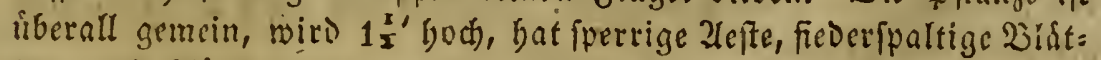
ter, und blügt das gar:je Jagr gindurw.

2. STromung. SSiliqnosa. Mit Yơngeren, runblicken nocr effigen, vielfamigen Sdyoten. Daltut: 
Der Robl (Brassica oleracoa), mit orcfrunder, langer Smote, auf welder ber Griffel als ein Eurzer Jortfás fteben bleibt; Samen in einer Reife, aber abwedfelno an beiden Nisthen befeftigt. Dis vielfachen 21barten Diefer aus Dem füoliden (Europa ftammenden Dilans ze werden in allen Sjatten gebauet, und als Jemúfe gegefien. Der $\Re$ a p $=$ oder $\Re$ ú $b\{a$ me $n$ (Br. napus) bat eine fpindelformige

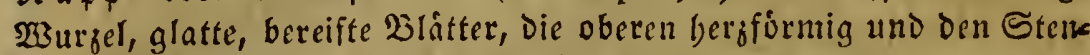
gel umfaffend, unD Die unteren leierformig. Bemcin an Wegen und auf Felbern; wirt angebauet wegen Der ilhaltigen Salnen, aus welden

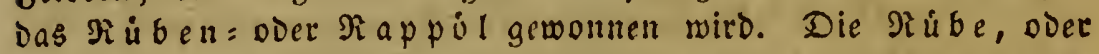

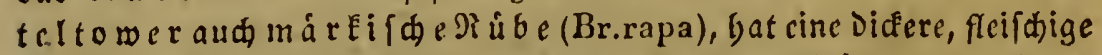
23urzel, nidht bereifte, leierfórmige, raube Murzelblátter und glatte, ungetbeilte Etengelblátter. Wegen Der ef́baren Wurzeln bauet man fie an, am beften im lolen Gandboden.

Der $\mathfrak{R}$ ettig oder Das $\mathfrak{R}$ a $D$ i \& đen (Rhaphanus sativus) bat cine Difere, baudhige, geglicberte, runde, inwendig mit leeren Neben. fäbern verfegene Sdjote; grofie, raube, leierformige Dlatter uno weife Slumen. Die fleifhige Laruel wirb gegeffen.

Der $\subseteq \mathfrak{e} \mathfrak{f}$ (Sinapis) hat cine nidt febr lange, mit \&angeftreio fen verfebene, gefdnabelte Edote. Der we if́ $\mathcal{S}$. (S. alba) bat fefr Eurge, baarige Edoten; Der id wa $\mathfrak{r}_{b} e$ S. (S. nigra), lingere, nadte. Deide werien gebaut, befonters in fiididen Gegenden, und liefern Dos gleidnamige Ejemuirz. Der fdroarje $\mathbb{S}$. ift fharfer und mird befonders jul Dlafenpflaftern benutzt.

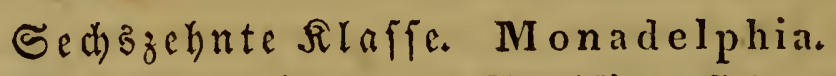

§. 155. Die glcich Inngen Stantbfibon find unter fich am (Sirunde verwach) 5 en, tmo bilden einen Ring ober (Sylin= ber um bie Griffel. Die Sronungen, bier wie it ben beiben folgenden Rlaffer, nach Der Bahl ber Staubgefóse.

1. Sronung. Pentandria. Nit fünf Staubgefifizen.

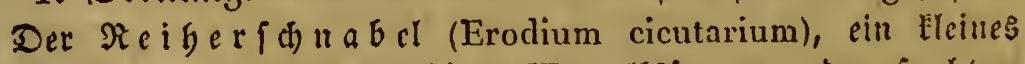

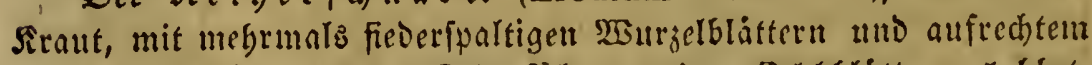
traubigem Blútbenidaft. Sebe Blume mit 5 Sieldblattern, 5 blut:

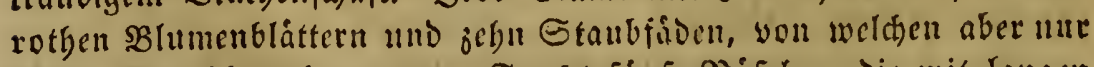
finf Etaubbentel tragen. Frudst fünf Saififden, Die mit Tangen

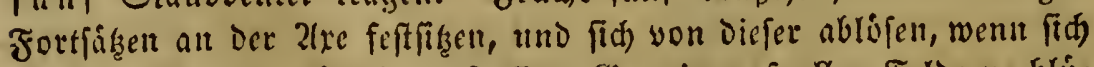
Der Fortfas fpiralformig aufroll. Gemein auf allen Fildern, Glito bet zeitig itt Frubiabr.

2. Sronung. Decandria. Mit jebn Staubgefápen.

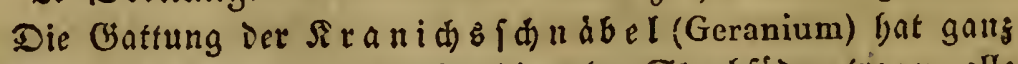
bie Senngeiden Der vorigen, aber Die befn Gtaubfiden tragen alle 
Staubbeutel. Dran hat bei uns viele Zurten, unter welden fid vlete Durd einen eigentbümliden उerud auszeidnen; $\dot{8}$. $\mathfrak{B}$. G. robertianum, Blittjenf haft mit bwei Eleinen, rotben Blumen, Blätter Dreic

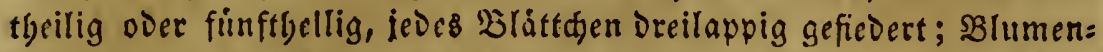
blátter ogne 2lusfdnitt, Sieldblätter mit einer B̧ranne. Besmein in

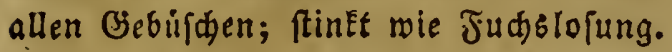

3. Dronung. Polyandria. Mit vielen Staubgefä́áen.

Sjierber Die Familie Der $\mathfrak{M}$ a I ve nn $\mathrm{gew}$ á 由 fe (Malvaceae), bei welder bie vielen Staubfäben einen Didfen (Sylinder bilden, aus

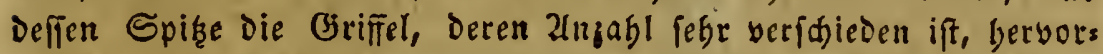

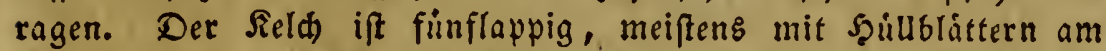
Srunde (äuferer Sield)); Die BiumenErone fünfbláttrig, Blätter am Grunde sufammenbängend. Die Frudt beffegt theils aus vielen ein= famigen in einen fireis um Den Frudtboden geffelten 21 denien, theils aus einer megrelappigen, megrfádgerigen, yielfamigen Siapfer. $3 . \mathfrak{B}$.

Dle $\mathfrak{B}$ a uerrofe (Althea rosea), mit fúnf: oder mebrlappis gem, ăuferem Seld, vielen biriffeln uno vielen einfamigen 2ldenien in einem Ringe. (Eine anfrewte, mefriálfrige Etaude, mit grofien, rauben, fünflappigen Blättern, und groféen àbrenförmig geftellten,

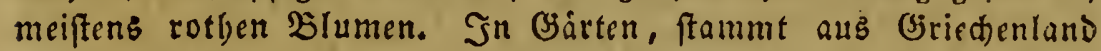

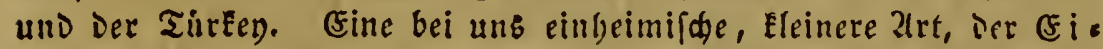

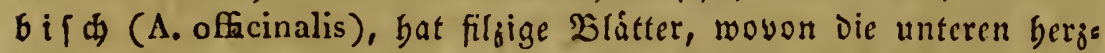
fơrmig, Die oberen lánglid eifúrmig, undeutlid Dreilappig find, und

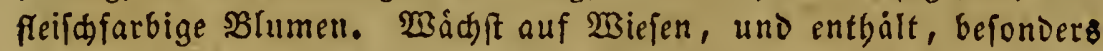

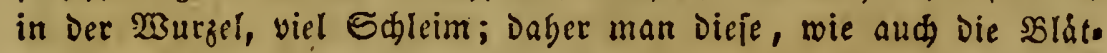
ter, gुum Brufttbee benuşt.

Die $\mathfrak{B}$ a u m w ollenpflange (Gossypium herbaceum) bat einen Dreibrättrigen, gezágnten, áuferen Sield und eiren cinblättrí. gen bederförmigen, inneren; Jrudt eine DreiÉlappige, Dreifádherige

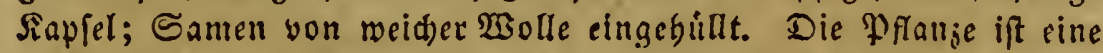
má̈bige Staude mit fperrigen Weften, fünflappigen Blâttern, Deren Deittelmerve einen Drífenfleck bat, uno gelben Blumen mit violet:

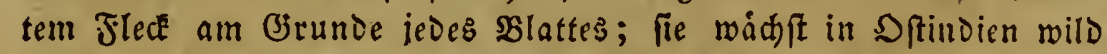
und wiro aud angebauet, da ble wollige Saúlle bes Gamens die bes Eannte Baummolle liefert.

Siebenjehnte đlaffe. Diadelphia.

§. 156. Dic gleidh langen Staubgefáne fint in jwei Bündel mit cinander verwachjen. Die $2 \mathfrak{C n}_{j}$ ahl ber Staubge= fäpe giebt bie Rennzeichen Der Sronungen.

1. Sronung. Hexandria. Mit je dhs Staubgefáp̃en.

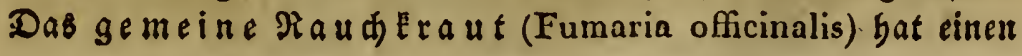


gmeibliftrigen, blufädtgen Seld; cine vicrblattrige, radacuformige BlumenEtone, Dereh oberes SBlatt am Grunde in elnen ftumpien

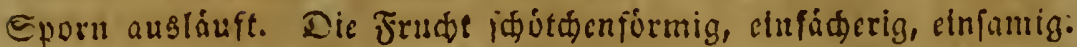
Dic Pfanze wadef bei uns auf Fornfeloern, bat cinen ctwas nieder: liegenden Etengel, Dreimalgefrederte Blätter, Deren Eeilformige \$lktt= Фen wieder getbeilt find, und cinen dgrenformigen slitbenftand; Die Slumen fino fleifकfarben, mit DunElcrer 2iúnoung.

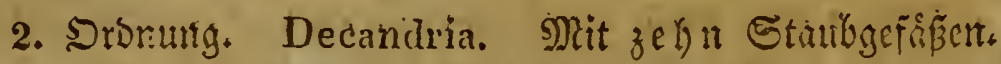

Dicfe Sronung biltet ciue felft grofie Janilie, bie unter Dent Siamen ber Sallfenpllanjen (Leguminosae) oicr Sdmetter: lingsbiumen (Papilionaceae) bekannt ift. (Fe find thcils baums,

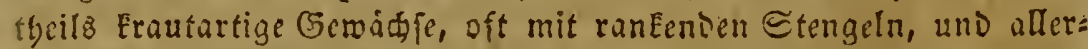
meipe einfaç gerieocrten, feltener gefingert geñederten Slätern. Die şlumen fechen in oen Blattudfirln, oder aln Errie, und biloen zrau= ben, Sinipfe, oder fechen paurmeib. Jeie bat einen cinfaden, finf: zibrigen Sild uno fú $\mathrm{n}$ S uno Grofe baben; Dab fúnfte, unpaare, oberfe Slaft ift greffer und ftegt mififen $B$ aufredt, man nennt es Segel (vexillum), Die bei=

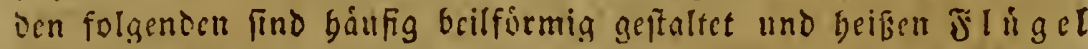
(alac), ite beiten unterfen verwadsfen meiftens zu einer siinne, die Den Tramen Riel (carina) erbált. In Diefar licgen Etempel uns Etaubfáden. Die leşteren bitoen eincn Eylinder um jenen, mo baangen theils alle, tbeits bis auf einen, unfer fit bufammen; dies fer cine ift inmer ber oberfte. Die orudt if eine bocielappige Sgille, in weldace bie grofien Eamen an einer Tath frizen. Sielé Dpranjen Diefar Jamilie Find midtig; $\mathfrak{z}$. $\mathfrak{Z}$.

Die $\mathfrak{B}$ ognen (Phaseoli); allermeift rankente Wfanjen, mit ab=

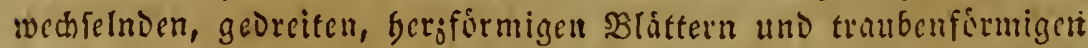
Blumen, Deren Siel mit Den હtaubfácen uno Dem Stempel fpiral: formig aufgerollt ift. 'Stulfen lang, grof; Samen nicrenformig. Dian bauet bei uns zwei 3 trten: Die ङ bog ne (Ph. vulgaris), mit rankendem Etengel und gejuciten Blu=

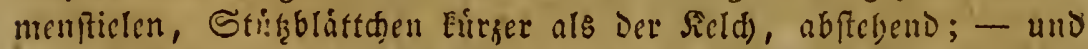
Die 3 werg: oder $\mathfrak{Z} \mathfrak{e r d b}$ olg ne (Ph. rianus), mit aufechtem, glat= tcm Stengel und Etriţblättd)en, Die liber Dell Feld binanšragcti.

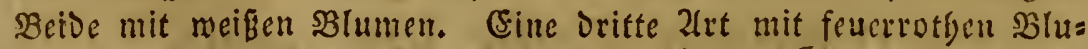
men und geffecketem, gróperen Samen (Ph. multiflorus), Die aus

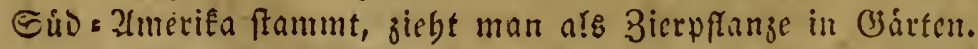

Die (E) $\mathfrak{r}$ be (Pisum sativum) bat aud ranfende Etengel und ibeiparig gefieserte, in sine gianEe außlaufende Blatter. BSlumen: fitile vielbrumig, in Den Blattadfern, mit meifen sBrithen. Eame runolich. Lleberall angebunet feit langer 3eit, Saber man Das eis gentfidye Saterfand Det (Sibfe nidht melge Eennt. 


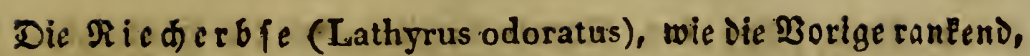
mit adfelftándigen, zrweiblumigen Blithenftielen uno in Pianten aus=

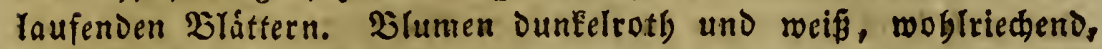
mit breitem Seget uno ungleidl langen Sielwzägnen. Zlus Epanien, bei นทร ałs Bierpflanze in Çårten.

Die WBickeng attung (Vicia) bat einen fünfóbnigen Feld,

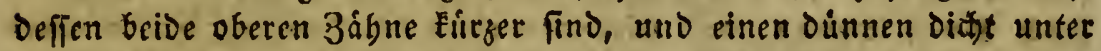
Der Tiarbe mit einem Syaaridgopf verfegenen briffel. Saúlfe langgez ftreckt, vielfamig. Nan bautet bei uns 2 2trten: bie Sa atwi de (V. sativa), mit rankig gefiederten Bríttern und adblelftán’igen

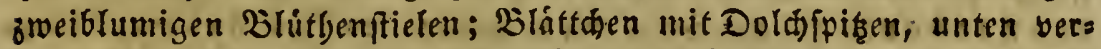
Eebrt berffírmig, oben lánglid eifórmig, Szúlfen baarig; - uno bie Saubobne (Vicia faba), mit aufredtem, Difém Etengel und nidft rankig gefieberten $\mathfrak{B l a ́ t t e r n ; ~ B l a ̂ t t d e n ~ , ~ e i f o ́ r m i g , ~ g l a t t . ~}: \mathfrak{B l u =}$ men zu orei; Szúlfen Jaarig, befonders iumendig; Samen grof, nierenforrmig. Stammt yom Easpifínen Meere.

Die Rinfe (Errum lens), mit gleidzígnigem Reld) und auf: reditem, Liberall baarigem Briffel. Şúlfen wenig $(2-5$ s) famig, Samen zufammengeorickt. Stumen ie zmei, den Blattern gegens sberfeteneno, weif; ftammt aus dem füblichen Europa... Sis ....

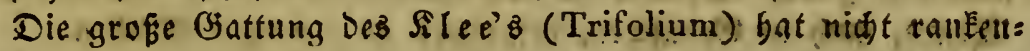
De Etengel, geoveite Blätter und einen rundliden, Enopffórmigen

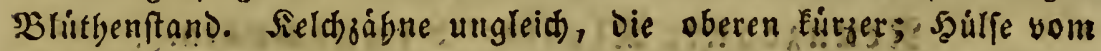

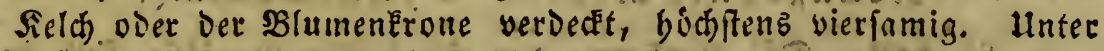
Den yielen Zliten ift $\mathrm{Tr}$. repens, mit weifer, ftehenbleibender $\mathfrak{B} / \mathrm{lu}=$

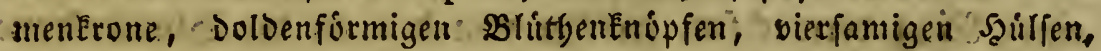

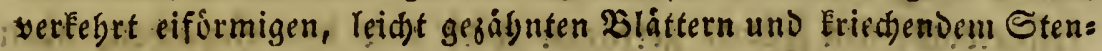
get, eine ber gemeinfter. Heberall auf Stoppelfertern und owifhen Dem Setreide.

\section{2ditzehnte Slafie. Polyadelphia.}

§. 15\%: Die vielen, gleich langen Staubforen ftehen mit Den Stempeln auf Demfelben Frudbtboden uno fint in

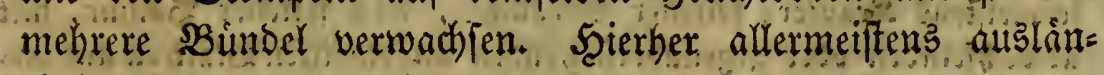
bifche (Sattungen; 8. $\mathfrak{B}$.

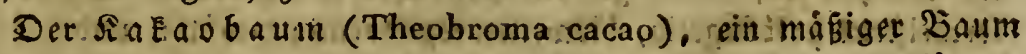

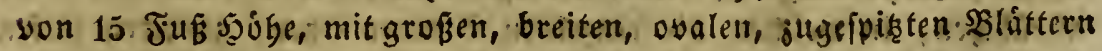

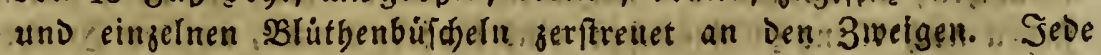

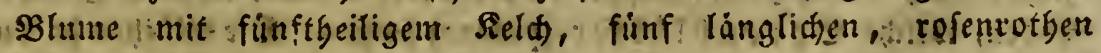

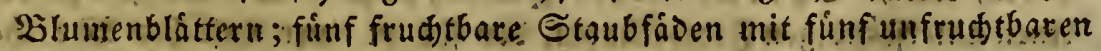

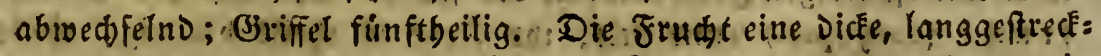

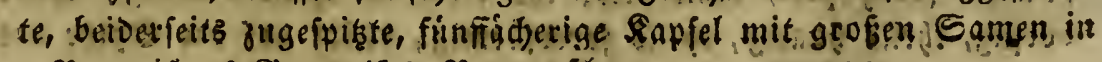

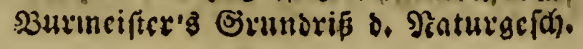


sinein \$ret. 2lus Den Samen bereitet man Die Bgoeolade. Der Baum whd) im beifen 2(merika (befonders Dierito) will, wirb abee aud Dort angebauet in idhattigen Waldern:

Die Orangeng a tung (Citrus) gat einen oreis bis fünfzábs ulgen Sield, 5 slumenblatter, und viele in mebrere Bindel verivad. fene Staubfabon; Die Frudb ift mebrfáderig, faftig, entbált in jebem Jach $2-3$ Samen, und wiro von einer lederartigen, orúfenteiden Saut belleibet. 3wei 2lrten find befonders roidtig: Der Po mmes rangenb a u in (C. aurantium), mit gefligelten $\mathfrak{B l a t t}$ tielen uno Eus geligen, in Der \&äugzadfe zufammengebrudêten Frudaten; uno Der $\mathcal{S}_{\text {is }}$ tr on e 11 ba um (C. medica), mit ungefígelten Dlattfielen uno láng: lid) eirunden, an jebem Dol gugefpişten Frudten. SBeide ftammen alts 2fiten, werden feit langet Beit in Jtalien und Eúbicuropa ans gepflangt, aber aud bei unz in Treibbåufern gezogen.

Cinbeimifh ift aus Diefer Slaffe nur Die Hartung Des Sob a me nis erauts (Hyperieum), mit fúnflapplgem Reld, fünf 98 lumen. blättern unD 3-5 Staubfábenbindeln, Frudt 3-3 fäderig, mit vie Ien Gamen am mittleren Duttertuden. Semein ift H.perforatum, mit gegenúberftebenden, oval langettforaigen, gelb punctirten $\mathfrak{B l a ́}$ s: tern und gelben Blumen in Den Slattadjeln und am Ende. 2/n \$3egen, aud in Bebufden, befonderz an founigen Etellen.

Reunzebnte Rla!fe. Syngenesia.

§. 158. Die 2utheren ber funf Staubgefäse find in einen Ring verwach fen, weldher von ben an ber $B$ lumenterone feftgemachfenen faben getragen wirb und ben einfachen, oben in 2 piralig eingerollte Fortfise getheilten, Siriffel umgiebt.

(Es bilbet Diele ganze \$lafie eine einzige Familie, bie unter dem Tamen Der. zu fa m me n gefełten $\mathfrak{B}$ I u men (Compositae) betannt ift. Sie bat ben groften' umfang unter atlen; indein ber o wilfte Thetl aller belannten Prangen uno uber 300 Bastungen bierber gee Gören. Folgendes fino ifre gemeinfamen Mierêmable: - Ee fino als lermeiftens Reduter, feltener Steảuber, nod feltener Baume, mit abmedfelnden ober gegenúberftebenden ungetbeilten 28 láttern und vies len Blüthen auf einem gemeinfdaftliden Frudbrboben, Diefer ift als lermeiften's balblugelig, bismeilen kegelformig gemólbt, uno bat klcine

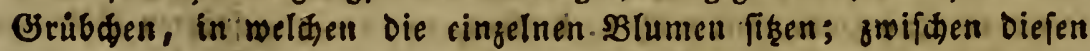

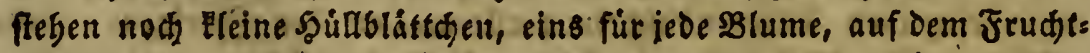
boben, welde espren blátter (paleac) beisen. Die Blúthen baben cinen unteritänoigen Frudthtioten, Der fid zu ciner einfaden $\mathfrak{W}_{\mathrm{B}} \mathrm{i}$ (d)= frudt (caryopsis) entwidelt; igr Seld' ift theilb blattrig, theils borftig, -theits gaarig, theils federformig, bleibt auf bee Spthe Des 
reifen Samens fteben, und bitdet die Sa InenÉ rone (pappus). Die Blumenfrone ift einblättrig, róbrig, theile regetmáßig fúnflappig, theils in eineir buirgenformigen Qappen verld́ngert. Naad) Der Strís

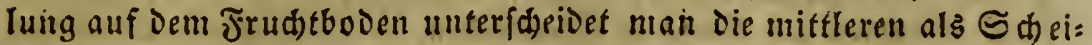

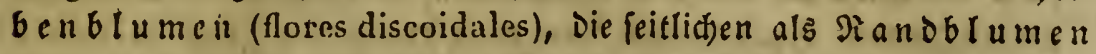
(flor. marginales) oder Strablenblumen (fl. radiantes), wenn fie zungenformig gebiloct find. Der gemeinfda aftlide Frudtboden bat

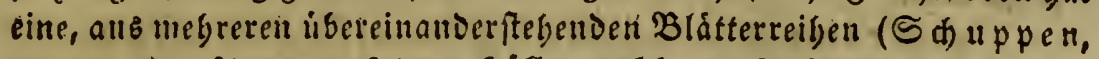
squämae) jufaumengeferçte, salille, welde anthodium, calys communis oocr periclinium genannt wiro.

Lin he theilte biefe filaffe in 4 Dronungen.

1. Sibmung. A equalis. Die Stheiben s und Mandblu= men Gaben gleid)e (Seftalt und gleichen Bau. 2fub allen ent= widkeln jich reife Sanient.

a: 2llle SBumentronen íegetmábig fünflappig. Cynareae.

Die Diftelgattung (Carduus) bat eite borftig baarige Sa: mentrone uno Şüllenfouppen; die in fteife Etadelin aušlaufen. Frubtboden borftig. Ilnter megreren bei uns einbeimifden 21 rten ift C: nutans, mit berablaufenden am Rande Dornigen Bráttern und bäingenden Blüntenfipfen, Deren Sgullenfduppenffadeln fegr groß̈, weitabiltcheno und lanjettfoemig find, eine Der gemeinften. 2uf Jels Dern, an Tiegen :c.

Die Sile tte (Arctium Jappa) hat ben Enopffurmigen Blutbens

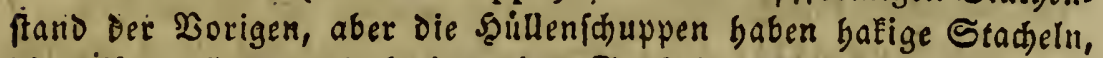
Die Biläffer find gró̧, breit, ofjue Stadeln, aber fitzig. Heberall

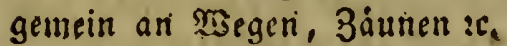

b. zlthe Błtumenktonen zungenförmig. Cichoreac.

Der \&úmell a gat ober bie $\mathfrak{B}$ utterblume (Leontodon Tarasàcum) bat einen unzertbeilten $S_{d}$ aft, Defien Blütbenboden yon einer vielblattrigen şülle umgeben ift. Der Boben ift nackt, und

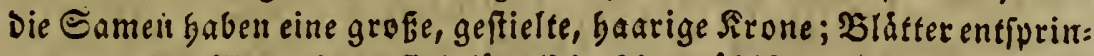
gen aus Der $\mathfrak{W u r}$ el uno find länglid, fárotzáanig. Lleberall gemcin.

Die (E) id orie (Cichorium Intybus) bat einen vergrweigten Sten: get, mit bimmelblauen siluthenenúpfen in Den Blattadfeln, Deren Şülle aus einer Doppelten Slätterreibe befteht. Frudhtboden mit

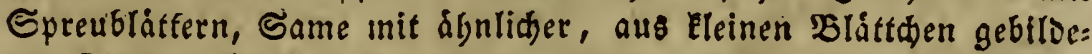
ter Frone. Hebarall gemein an Wegen; wird wegen Der grofen fleifdigen $\mathfrak{k}$ urgel, Die man geróftet uno gemablent unter Den fiaffe

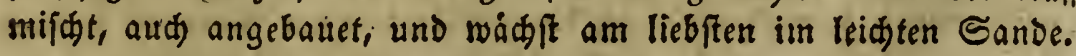

2. Sronung. Superflua. Scheibenblumen regelmás̄ig

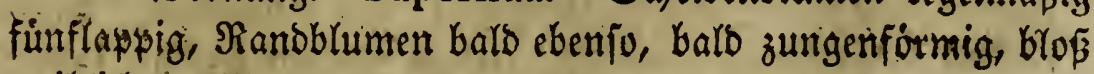
weiblicf; 2ale geben mife, volftommene Samen. 
a. ?llle Slumen regelmáßig, fünflappig.

Der Rainfarien (Tanacetum vulgare), eine ftaubenartige Difanje unit-Doppéttifederfpaltigen SBlattern, Deren Lappen gejáljut fino. Slumen au Ende in 2fferDolden, gelb; Satllblíttchen Dadbie= gelartig, runo. Pinnoblumen Elein, meift Dreilappig, Frudtboden nadf. SamenErone febr Elein. B̈emcin in Bebuifhen, an Giráben, Jrifien :c.

Der $\mathfrak{B}$ er ut utg (Artemisia Absinthium) bat einen aufredten afti= gen Etengel mit weifigrauen, oreimal ficterfpaltigen Blätern; Blu= menEnipfe rundid, nickeno; Jruchlboden nackt, Şille Dachjiegelfors mig fouppig, SamenErone feblt. Sidt felten an Megen, auf Jieg= riditgaufen u. f. w.

b. Mit grofien, zungenformigen Strablblumen. Radiatac.

Die Bf ar ten after (Aster chinensis), eine cinjägrige Staule mit wenigen fperrigen 3 meigen, Die am (Enbe einen grofien, flathen $\mathfrak{B l u}=$ menépf träen., Blumenbitlle fduppig, blattformig; Jrudtboden nackt; Ranoblumen verfdieden farbig, bläulid oder roth, bizmeilen meís; Brätter länglid lanjettformig, gejăbnt, flęeno, Die oberen ppatelformig. 2lus ŞGina, bei uns in Bärten als Bierpflange.

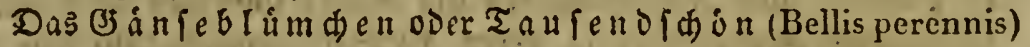
Gat elnen Eegelformigen, nackten Jruchtboden, eine frache, fouppige Sluttbentillte; weife Strablenblumen mit rotben Spizen und gelbe Sd)eibenblumen. Siatter fpatelformig, entfpringen aus Der $\mathfrak{B}$ urzel;

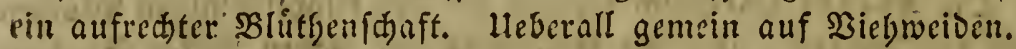

Die (Ga mille (Matricaria chamomilla) bat citten Eegrlfors

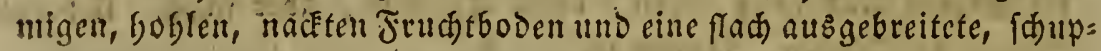

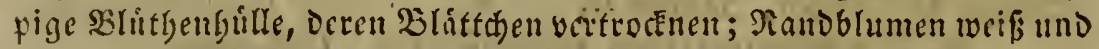
berabgangent. Dlatter smeimal ficoeripaltig utit linienformigen Euppen. Jaiufig auf Etoppelfeldern; 'wird als Szeilmittel benutzt.

Die $S$ a a figarbe (Achillca millcfolium) ift cine aufredit= ftebrnoe, 1' lobe 'tfranze, mit gefurdtem Etengel uno zweimalfie: Derfpaltigen Slaftern, Deren Rappen zugefpięt frno. Blittgenenipfe in 2lfterdoldern ain Ende Des Stengels; jeder mit fladem, you Spren= Glittern bevecEtem Frndtboden un gering an 3afl, alle meif. Stemein an Begen, liberall.

3. Sronung. Frustranea. Sat 3witterfcheibenblumen, bic Samentragen, uno unfruchtbare, weibliche Randblumen. Sabin:

Die fiornblu me oier Trem fe (Centaurea cyanus), mit Eu=

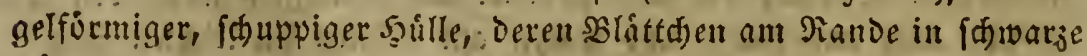
3ágne auslaufen; borftigem Jiudtboden und borftiger Samentrone. Siandbrumen robrig und gejaleditslos, nille blaigefárbt. Blatter famal, rangettformig, wie Die fperrigen Stengel mollig. Heberall zrifden Dem Getreide. 
4. Dronung. Necessaria. Mit bló̧ mánnlichen, regel= måpig fütrfappigen, róbrenfórmigen Sdheibenblumen uno weiblichen, zungenformigen Strablbrumen, welthe allein $\mathbb{S}_{\mathfrak{a}}=$ men tragen. Dabin:

Die $\mathfrak{i n}$ iel = ober To D tenblume (Calendula officinalis), mit nackstem, fradem Frudtboven, vielblätriger suille uno grofien,

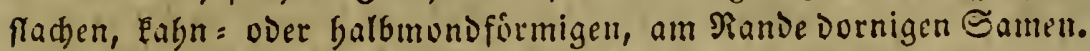

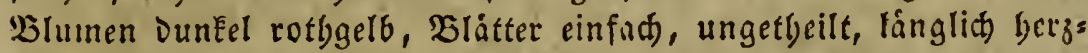

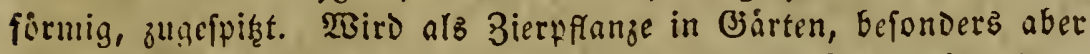
auf sirngbofen, angebauet, uno jum Ed)muck der Seiden benutzt,

\section{3wanzigfte Slaffe. Gynandria.}

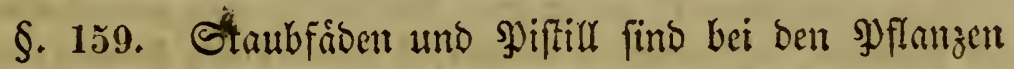
biefer 2rbthcilung in eine Såule vermachfen, welche auf bem Fruchtenoten rubet, und an ber bie $\mathfrak{R} a r b e$, und bariber voer Daneben bie Strubbeutel befeftigt fint.

1. Sronung. Monandria. Mit einem Staubgefáp.

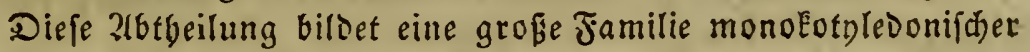
ऊemádfe, Die mit Dem Tamen der Saleppfla Deen (Orchideae) bezeidnet mirb. Zflle find Ernutartige Pflanjett

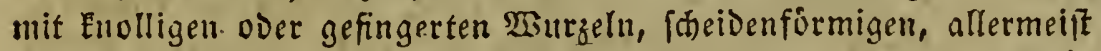
ungetbeilten, ovalen oDer lanjettformigen Bláttern, aufred)tem $\mathfrak{B l i}=$ thenfdaft, an weldem Die Blumen eine 2legre birden. Sede $\mathfrak{B l u m e}$

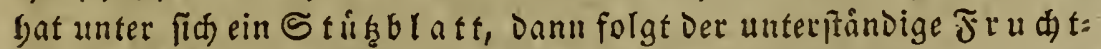
Enoten, und Diefer trágt Die unregelmåßige, fechbbríttrige, gefárbter

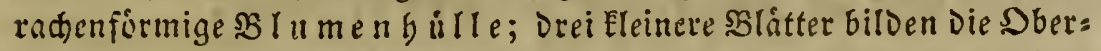
rippe, und yon diefen biegt fï Das mittlere liber Ias in cine Sáule

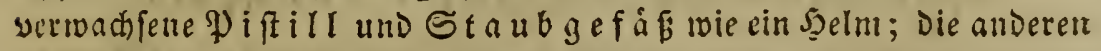
Drei bilden Die Lutterlippe, befoniers das mitftere grof́e, oft mert= wurroig geftaltete, meiffens in megrere Lappen getgeilte und gefpornte. Die Jrudit ift eine langgeftreckte, einfáderige Sapjel, in melder Die férr Écinen, vom Mantel umgebenen Samen an wandpänoigen, ftreis

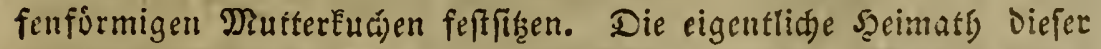

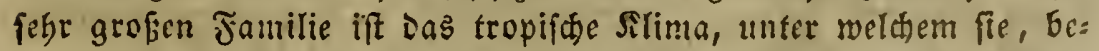

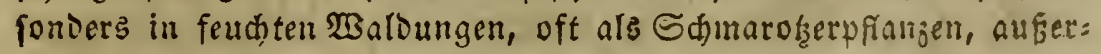
ordentlid gedeiben. Mande Diefer find auferoroentlid fajón. Sine

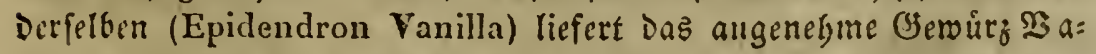
nille, weldes dic SamenEapfel ift; Die einfeimifaen wadjen vor:

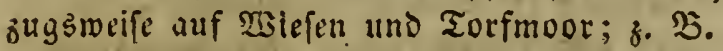

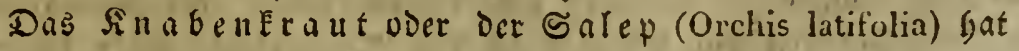

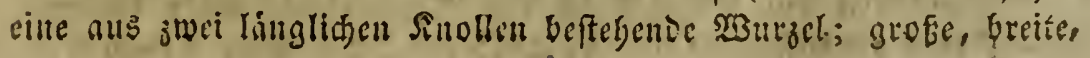


brunn gefledite Bhitter; sinẹ boblen Esaft uno bell blutrotbe Bin. men. Die Interlippe if grof, breit, Dreilappig, mit abmarts gebos genen Piändern und cinem Eurzen, abgerundeten Gporn am Birunde.

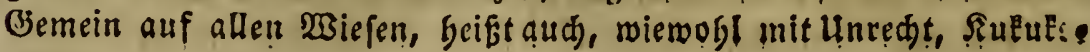
blume.

Bei Ophrys ovata baben Die oberen Blumenblatter faft gleide Osrofie, aber oas mittlere untere (Unterlippe) iff febr lang, breit, gas belformig gefpalten und bat Eeinen Eporn; Die Pflange bat eine

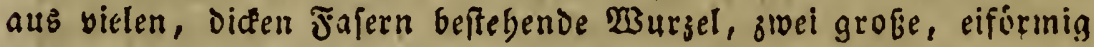
Ereisrunde Blátter und einen aufredten Bluttgen ldaft, mit viẹlen grinen Blumen. Sadufig in Bebuifden.

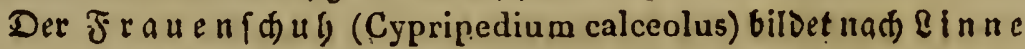
eite befondere Sronung, Da er jmei Ștaubfáden bat, gebirt aber in Diefe Jamilie. Die gelbe Unterlippe ift groff, breit, baudig, oben

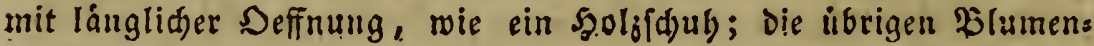
blätter fino grade, langettformig, grofi, und baben cine brautrotbe Farbe. Stengelblätter breit, eifórmig, zugefpiçt; $\mathfrak{B u r z e l ~ f a f e r i g . ~}$ J̦! Gebirgsggegenden des mittleren Deutfalandṣ.

2. Sronung. Hexandria. Mit fech 3 Staubgefápen. Dibitit:

Die S fterluget (Aristolochia clematitis), eine Eluutartige Dranje, mit abred)ieluben, fiemlich grofen, beraformigen Blattern. Blumen einige in den b!attád $\{$ eln, jede auf einem befonderen Stiel, nit unterftandigem FrndtEnoten, uno einfader, einblittriger, tuten: firmiger, am O3runbe baudhig ermeiterter, gelber Slumenbülle. In Diefer Ermeiterung oie Starbe, mit feds 3millingsftaubbeuteln in if: rem Ilmfange. 2ln 3äunen, aud in Bárten zu Lauben?.

\section{Einunozwanzigfte Alaffe. Monoecia.}

§. 160. Linne rechnete bierher alle Sllangen, bei wels d)en bie Staubgefäe und Stempel nicht auf ocmielben Frutht= boden, fondern in ganz verfhiebenen slumen ftehen, beren peridiebene, minnliche und weibliche, Blumen aber von bem= feltsen Stamm getragen werden; im Deutichen pflegt man fie einhåufige Sflanjen ju nennen. Die Dronungen ferste Rinne nad) ber $2(n j a b l$ der Staubfáben feft. Folgende $F a$. milien ftehen bier:

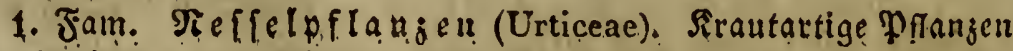

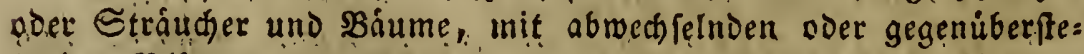
henden Blattern uno Blumen in Den slattadfeln; beide Eäzden= furmig ober gebduft, mit $\overline{4}-5$ : lappigem fiel $\phi$, olne Blumenfro 
ne, und fo vicl Staubgefágen als Reldiappen. Frudit eln trodencs - ber fleifóiges 2ldenium. Dabin:

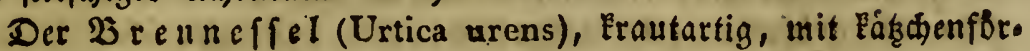
migem Blìthenftande, Seld 4 slappig, 4 Ctaubgefdée um ein be:

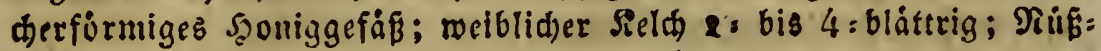
den yom Sielde ummgulat. SSlátter gegeniberftebend, ellipsifh, ftarE gejágnt, mit fratien, fteifen, Gremuenden Şaaren befę̧t. Lleberall gemein.

Der Na a lbeerbaum (Morus nigra), grofer Baum, mit berf=

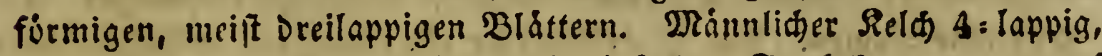
mit 4 Staubfíden, weiblidser mit einfactem Frudtenoten, worauf

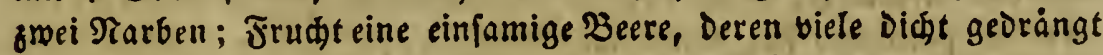
auf Demfelben Frudtboden ftegen, fdrwarg. Sei uns angep fanzt jur Siultur Desิ EeiDenmurmes; ftammt aus (Shina.

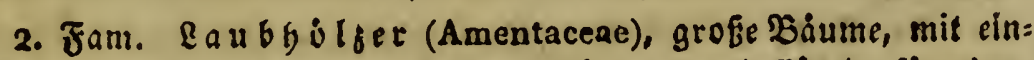

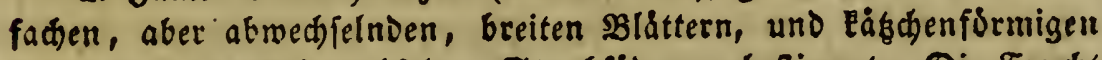
Blittfen in den Blattadfeln. Etaubfaden unbeftimmt. Die Jrudt

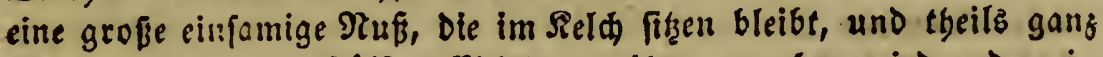
(Budde), theils zur Şälfte (Eidfe) von ihm ungeben wird; ober ein 3apfen, unter Defien GoIzigen Sduppen ein ober zrei Eletne gefli: gelte 2ldecrien fteden (Birlen).

Die (Ei d)engattung (Quercus) bat Tange gedegnte mánnlide

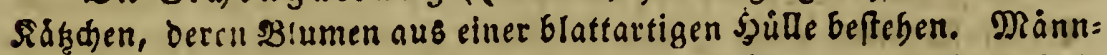

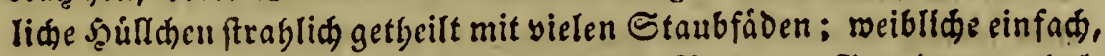

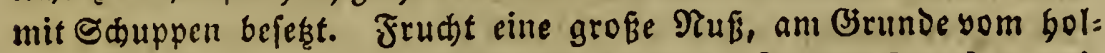
bigen Räpften ungeben. Nan bat bet uns 2 2trten: Q. robur, mit gríseren, grlappten Blättern, uno gróferen, ungeftielten Rúfien ; Q. pedunculata, mit Eleineren, nod) tiefer gelappten Slättern und Der

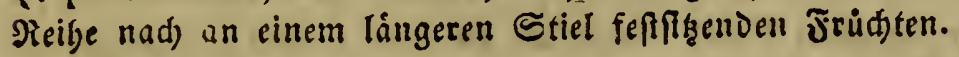

Die $S_{2}$ a fel $\mathfrak{n} \mathfrak{E}$ (Corylus avellana), ein holgiger Etraud, mif

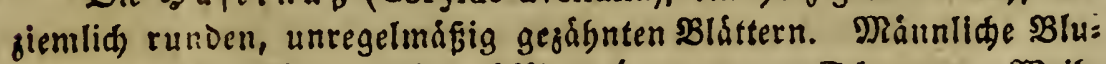

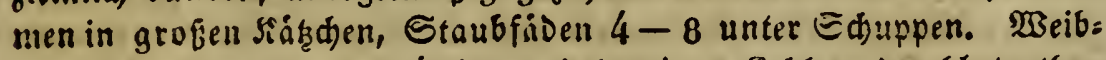
lide Blumen gu 3 oder 4, ieve mit lappigem Sield) uno 2 blutrothen

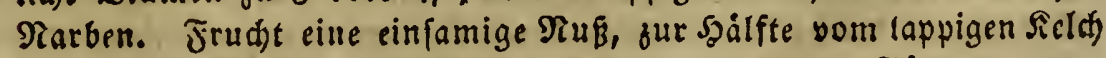
umgeben. Hrberall in Bäloern, und angepflanż in Bärten.

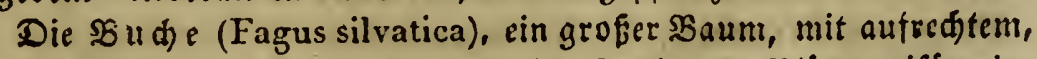
glattent Stain:a uno faft horizontalen 3meigen. Blätter eifurmig, ganzransig; männlidfe Blumenbitlle fủnflappig mit vielen Etaubfá: Den; weiblid, Ślumengúlle vierbláttrig, weidftadelig, mit getei orei=

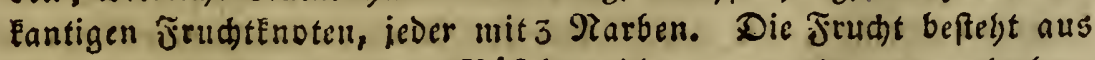
owei eimfamigen, Dreiedtigen giúfiden, Die ganz yon Der nummelyr bar: ten, golgiger slumengúlle unịglofien find.

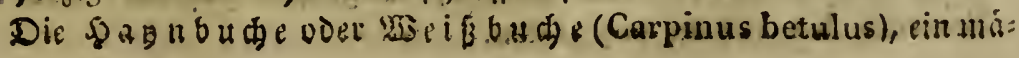


figer $\mathfrak{B a u m}$ mit eifómnigen, gezałgnten Blattern. Blumen in Siáts= đen, männlide gróber, unter Sduppen 10-20.Staubfáben; meib. lide mit groferen, gefpaltenen Sduppen, und unter jeder 2 Frudit: noten mit gabeliger, rotber Sarbe. Frudt eitle gefurdte Stuf, von Der großsen, Dreilappigen Sduppe leid)t eingebullt.

Die $\mathfrak{B}$ ir e (Betula) ift baumartig, mit dumnen, fegr fdlanken

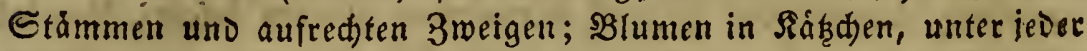
Eduppe Der mánnliden mebrere Elcinere Sduppden mit eitrer 3 willa lingsanthere; meiblide Siáçden borjig, unter jeder Sduppe orei

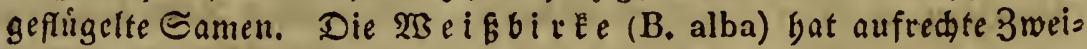

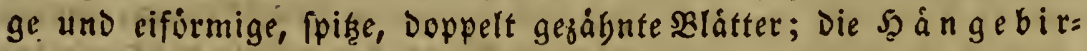
Ee (B. pendula) bat binne am Ende Gerabbangende 3 weige, mebr oreifeitige Blátter, und längere, zugefpikete Schuppen Der weiblidipu Siázhen.

Die (Erle oder E I Ter (Alnus glutinosa) Gat gang Die fiennzcia den Der Birke, aber unter Den Sduppen Der mánnlidhen Siảazchen fteben viev vierlappige Sduppden, jeve mit vier Staubfáden; meib: lide Sduppen mit zwei Frudtenoten, woraus eine jweifäherige, in iebem Jad einfamige Faplel. Der Danm liebt feudfte Standorte, Daber in sbrúden, hat einen graden Stamm und faft feilformige, unten in Den 2dhfcln Der Rippen baarige, jung flebrige Blátter.

3. Jam. Na o elbolzer oder 3 apfen baume (Coniterae). ebenfallz grobesciume, mit gradem, bobem Stamm, und aliermeift quirlformig geftellten 3 weigen. Blótter rmolid, (4)mal, nabelfors

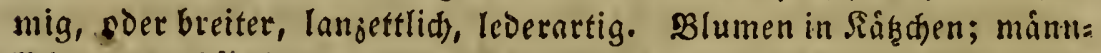
lidbe unter bäutigen, trodenen, weiblide unter.bolzigen Schuppen, weld) Gernad Den bartew, Eegel = oder Eugelformigen Zapfen bildert. Dabin:

Dic Öattung Pinus, Eenntlid an Den grofenen, Eegelfirmigen 3apfen, Deren ङauppen Dick und aufgeroorfen find, und an Den in einen Búndel verwadjfenen Staubfáden. Blätter febr fdamal, lang, siun, fpis, baufenmeis geftellt. Dabin: Die Riefer oder Fobre (P. silvestris), mit ie zwei ftraffen unterbalb gerwilbten Sliftern uno eingeluen $3 a p f e n$, Deren Sduppen rantenformig abges ftutzt find. Lleberall als $\mathfrak{W a l D b a u m}$, befonders im Siorden; liefert כau = uno Zrentbolz, Ther, Terpentlinisl, Eolophonium und Ped. - Die Pi nie (P. pinea) ebenfalls mit je. zrei şíttern, die iung baarig find, und fegr grofen $3 a p f e n$, Deren Schuppen am Ende abgerundet find und ftaré vortreten. Ciid = Europa; die Samen werden gegeffell, Die 3apfen von Den 2lten zu Sachantenftaben bes

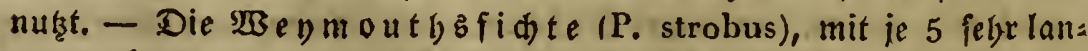

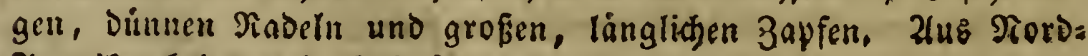
Imeriea, bei uแs in Suftgårten. 
Die Battung Abies bat lingere, geftrectetere, gleidmánfiger Didé Bapfen, mit Dinneren, glatten Sdauppen. Die Blátter jerftrent, olgne beffimmte Dronung, ober fegr didgt und zaglreich in sBifdeln, allermeift etwas breiter. Samen reifen foneller. Dabin: Die $\mathcal{S}_{e}$ : Der (A. cedrus), Brátter fraff, fpiłs, buifoelmeis; Bapfen elliptifo, Sduppen angeoriaft und abgefruţt. Grofer Daum, Der ein unges beures 2llter erreight und befonders auf Dem Ribanon wádff. Sgol woblricteno, zu Bleifedern. - Der $\mathbb{a} a \operatorname{rdg} e n b a$ u m (A. larix), mit jäbrlid abfallenden, ftumpfen, búfdelwois geftellten Blättern uno

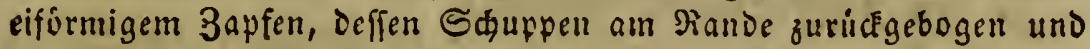
zerrifien find. Eud: Europla, bei uns einjeln in Zupftanzungen. Sadz

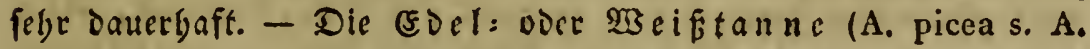

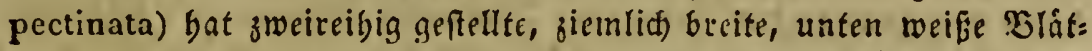
ter, uno aufrediftebende 3apfen mit ftumpfen, angedrickten Salup: pent. Megr fúdlid, bei unz nur einzeln angepflanzt. - Die $\mathfrak{R}$ ot th: zanne (A. excelsa. Pinus abies Linn.), mit viereçigen, fpişen, zweireibig berfitreut ftebenden Bláttern; Зapfen grof́, jángeno, mit

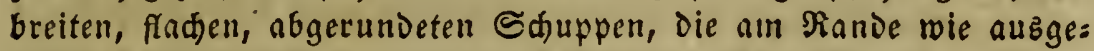
nagt erfdeinen. Befonders im nüroliden Europa, Sdweden, Finn= lano; liefert bie bojdften und beften Maftbáume.

\section{3weiundzivanzigfte Silaffe. Dioecia.}

§. 161. Die গুflanjen biefer NArnfe haben mit benen ber vorkergebenden grofe 2(er)nlicheit, aber unterfheiten fich

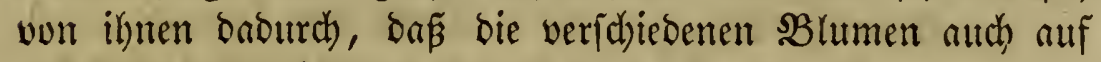
verichiedenen Stimmen ftelen, fo nelymlich, ba nur mannliche, bie anbere nur weibliche triggt. Sinne theilte biefe Slaffe ebenfalls nach der 3abl ber Stmubgefäfe in Sord= nungen. $\mathcal{D a}$ bie meiften mit in bie Framilien ber vorigen Slaffe geboren, fo weroen wir fie benen anreihen.

\section{3ut Den Utticeen gehort:}

Der $\mathfrak{a} \mathfrak{n} f$ (Cannabis sativa), eine einjifrige, fraubenartige

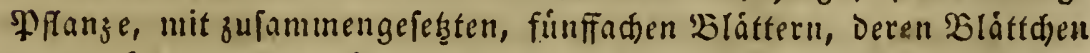

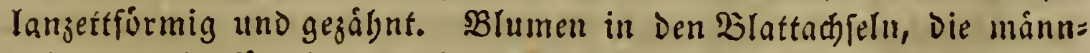
liduen traubenförmig mit fúnfbláttriget, griner $\mathfrak{B}$ lumenfülte und 5 Étaubfáden; weiblidge geuringt, unter Eduppen ein einfacher Jrudt: Enoten mit 2 Eolbigen Marben. Jrudjt ein ginisdeen. Heberall an= gebauet, anb Dem Stamm bereitet man Den saanf, Der ju Dieifen, Bindfaden, Tauen yerarbeitet wird; Camen geben Del. 
Der 5 op fen (Humulus lupulus), eine rankende, vieljąrige Stau: De, mit grofien, fürflappigen, gezábnten, gegeniberftegendell Bláttern uno adjelftindigen Blumen. Dläunlide in Trauben; Blumenbúlle fünfblátrig, grün; finf Staubgefäfe. WBeiblidbe Slumen bilden ei= nen 3 apfen, Defien ङduppen weid) und blattartig find; unter ieder Eduppe ein einfader Fruchtenoten mit gabeliger Siarbe. Salte ein stífden. TBild in $\mathfrak{B}$ áloern und B̈ebúfden, wird angebauet uno zum Marizen Des bieres Genutgt.

3ิ ben 3apfenbaumen geboren:

Der Ta u s b a m (Taxus baccata), ein máfiger baum mit Didht georángten 3weigen uno zerftreut ftebenden, fdmalen, linien: förmigen, zagefpikgten Dlättern. Mińnnlide Dlumen mit vierlappis gem Feld, 4 Slumenblattern und einem traubenformigen Etaubge:

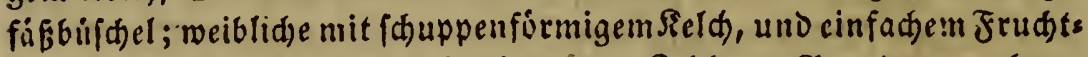

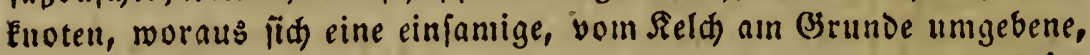
rotbe, fleifhige Beere bilbet. Sülid, bei uns anjepflanjt in biar:

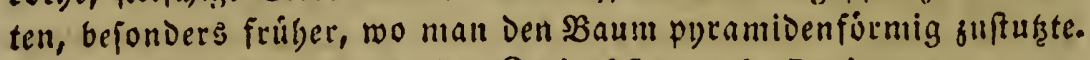

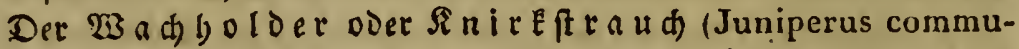
nis), bei uns ein Eleiner Straud mit niedergeirudten 2leften, und

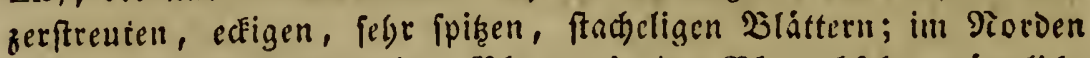
Europa's mege baumartig. Blumen in Den Blattadfell, mannlide zapfenfirmig, Die Eugelfömigen Staubbeutel am Diande Der Sdup: pen; weiblide ebenjo, Die unteren Sduppen leer, jwifhan Den bei= Den oberiten ein Fruchtelleten mit 3. গarben, Der fich fu einer 1-3= famigen Beere allzbildet.

\section{Figenthumliche Familien diefer Slaffe find folgende:}

1. Jam. Wre i o e $\mathfrak{b} \mathfrak{a} u$ me (Salicineae). Báume mit einfachen, abivedjfelnden 'Blattern; adjfelftandigen, kák̨d)enfórmigen Blitben, Deren Saillen blogie, blattartige Sduppen fins. Die Fridgte find viel[amige, langlide, ein = oder gmeifáderige אapleln mit vielen, Elei= nen, von feibenartigen Şaarell umgebenen Samen. Dafin:

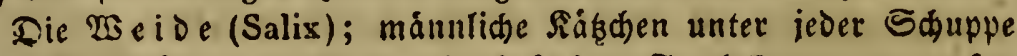
1-5 Staubfaden; weiblide mit einfadem Frudtenoten worauf 2 Tarben, Sapfel eiformig, Samen an Den Slappen befeptigt. Ilns ter Den vielen 3 rten Diefer Ojattung fino befonders bafufig; Die weif W3. (S. alba), mit lanzettfórmigen, bugefpişten, gefägten, beiderfeitz feidenbaarigen $\mathfrak{b l a ̈ t t e r n ~ u n d ~ g m e i t b e i l i g e n ~ \Re a r b e n . ~ H e b e r a l l ~ g e s ~}$ mein an Wegen, Durfern zc. - Die fi o.r b we i De (S. vininalis), mit febr langen, linear lanzettformigen, falt ganzrandigen bláttern, Die auf ber unteren Seite feidenbaarig fino. Die fiág̨henfduppen

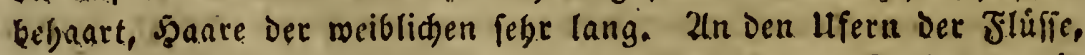

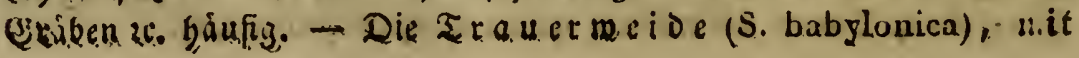


ferbe langen, bugefpikten, glatten biăttern uno Gerabgángenden 3weigell. Güblid, bel uns angepflanzt, befonders an Grábern.

Die pappel (Populus) bat alle Sicnnjeiden Der Meide, abee

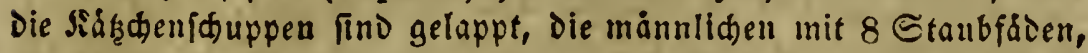
Die beiben Marben bismcilen zmeilappig. Maan Eennt bei unz mels= rere 2 rten, o. B. Die it a ll en ifda $\mathfrak{e}$. (P. dilatata), mit dreiecfigen, fpitzen, gezäbuten, beiberfeets glatten bláttern, Die breiter fino als lang. 3rweige aufredt, Daber Der ganze baum lang geftredit pyra= midenformig erfiteint. Stammt aus Dem nóroliden Jtalien, bei uns angepflanjt zu alteen; Die meiften biefigen Bäume fino mánnlide. Die 3itterpappel oder (Espe (P. tremula), mit rullden, fpigge=

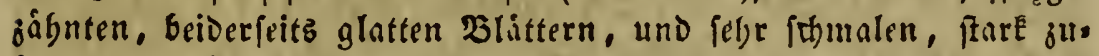
fammengedridten Stattftielen. Ebenfalls ein grofier Saum mit

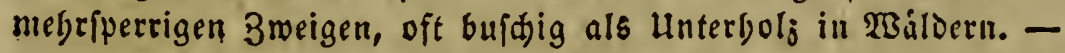

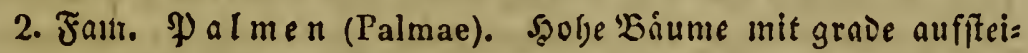
gendẹn, raubem oder getáfeltem, unverziveigtem Etamm, weldjer an feiner Epise melgrere grofie, langgeftielte, jufammengefetste, theils

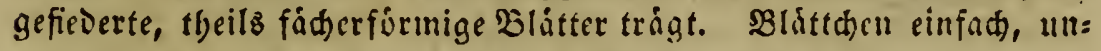
getbrilt. Silumen in Siolben, Mitspen oder Trauben mit fechsbláttrigem Derigonium in 2 gieiben, feds Etaubgefífen uno allermeipt irei

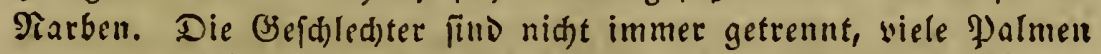
baben Die mánnliden uno meibliden Blumen auf Demfolben $\subseteq$ tamm, gegoren alfo in bie vorige filafíe, nod andere tragen die verfalicoe: nen $\mathfrak{B}$ lumen fogar in einer und Derfelben Traube (a norogy uifder Saul), menige endlid, abor arade Die midtigften uno beEannfeften,

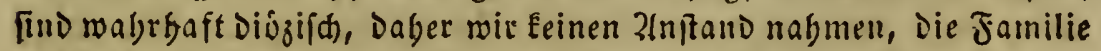

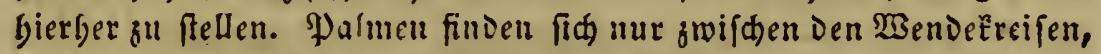
wadfen gemelniglid in Der Tiálje Dez. Tisafferz, Daber aur Etrande oder an Flufufern, und bilden Eeine Bálder, fondern fteben einjeln, büdftenz baufenmeis, am liebften zwifden Dem $\mathfrak{Q a n b}_{\text {ofl }}$ Der Trepen. 2tmerifa belgerbergt Die meiften. Widtige palmen fino:

Die Dattelpal me (Phoenix dact;lifera), Diózifh, blîtlen in Trauben grwifhen Dẹn blattipielen, Slátter gefievert, vieliodjig; Etengel ftadellug, Blättden Der gánge nadh gefaltet; Jrudte eine langgeftrefte, culinorifde, einfamige Єteinfrud, bie eqbar und woblfdmedend iff. 2(rabien und Tiord =2lfrika; Der gewibullidffe Frudtbauin jener Giegend, und für Die ármere Silaffe ein unentbels:

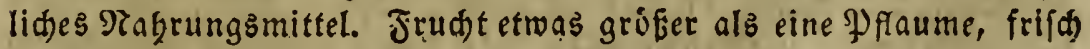
gelbroth, faftig. Der $\mathfrak{B a u m}$ wiro úber 200 Jabre alt, trigt im fedbsten Safre zuetift Frúdte und ift Dann wobl 12'. bod, ein alter. gegen 60 .

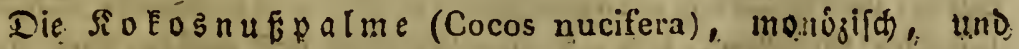

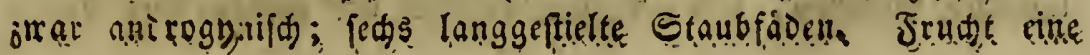




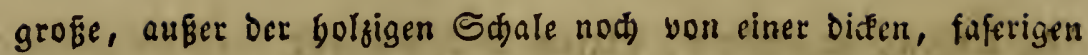

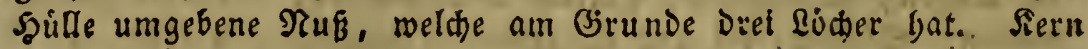
bobl, inmendig yol Mildfaft. (Ein bober Baum, mit vielen (gegen 20), langen, gefiederten Bláttern und vielen Sirifien, Deren jede 1' im LängşDurdmeffer gat. Ueberall zwifhen Den Tropen angepfrangt, vorjuglid auf ien Snfeln ocr Súdfee, und fur Die Beroobner Der: felben Daß midhtigfte Rabrungs mittel.

Die Delpalme (Elais guinecusis), ebenfalls monizifd, Slu= men in Folben, mit Doppeltem $6=$ blätrigem sperigonium; mánn= lide mit 6 verwadfenen Staubfaisen, weiblide mit 3 Siarben. Frudjt cine faftige Steinfrudbt yoll oben zugefpizter Form, Die in igrer

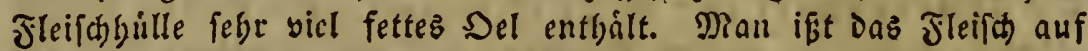
DroD geftriden wie butter, prefit Das Sel aus, uno benutzt es befonderz fur Bercitung von Eeife. Der Baum wiro gegen $30^{\prime}$ bod, ift uberall mit Den kurgen, am sande ftadeliden Stielen Der abgefallenen släter bedectet, uno trágt an ber Epiz̧e nur wenige, aber lebr lange, gefiederte, vieliodige SBlätter. Guinea, yon Da uad) $\mathfrak{s c f t}$ s Jubien verpflanzt.

\section{Dreiunozmanzigfte Rlaffe. Polygamia.}

§. 162. In biefer Slaffe follen, nach $\mathfrak{L}$ inne, alle bie= jenigen \$flanjen fteben, welche neben mannlichen ober weib= lichen Blumen noch Switterblitthen auf bemfelben Strmm tragen. (Fr theilte fie bann nach ber, 2(njabl ber Staubge: fâpe, wie die friberen Slnffen, in Srónungen. Da inders biefer Sharneter vielen 2 banderungen in ben verfhiedenen bierker gerechneten Spflanzengattungen unterliegt, fo baben viele paatere Botanifer biefe Slaffe ganz eingejogen uno die SJflm= zen nach ber 3abl ber Strubfíben in eine ber erften oreizelin Sinffen gebracht, andere bagegen haben nur wenige Spflanjen bier fteben laffen. Unter ben von $\mathbb{L}$ inne aufgefubrten cin= beimijchen finb folgenoe bemerkenswerth.

Die 21 bor ugattung (Acer) enthált baumartige Pfanzen mit rinfarben abcr gelappten sidttern, uno traubenformig geftellen, gi= yfclfändigen Dilumen, Die aus einem fünflappigen Feld), einer fünf: blattrigen Sironc, 8 Staubgefáfen, uno cinem einfaden, gefpaltenen Griffel befteben. Frudt ein Doppeltes, grofes, cinfeitig gefligeltes

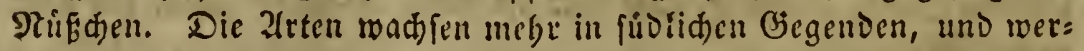
Den bei uns in Hnlagen angepflanzt; z. $\mathfrak{B}$. A. platanoides, mit fimf: lappigen Slattetu, Dcren Eappen unglcid tief gezábut uno unter= 
Galb weiflich grủn gefárbt find; Die jüngeren haarig. Blumen

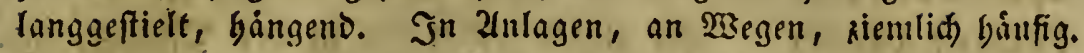
Wianche Zlten, mie Der 3 u de er a born ( $\Lambda$. saccharinum), entbal= ten einen fífen 3uckerfaft, Den man bemuşt.

Die (E) f (Fraxinus escelsior), ebenfalts ein bober פaum, mit einfadigefiederten 'Bláttern, Deren Blâtthen lanjettformig und gefágt, find. SBlumen in Trauben, iede Blittle bei biefer 2lat ofne Sield) uni firone, bei anderen. 2trten mit vierlappigem Seld und vierbláttriger Firone; ztwei lange Staubgefáfie, FrndthtEnoten mit zmei giarber. Frudft eine einfamige Jlingelfrudtht, mit aufredtem, gra= Dem, aut Ende fojief ausgerundetem Jlügel. SBei uns in Zlnlagen,

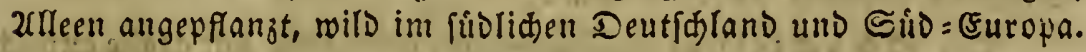
Dis Minde entbált einen bitteren Etoff.

Die Meldeng a ttung (Atriples) enthalt Erauturtige, aller= meif einiäbrige Dflanjen, mit gegeniberftebenten oder abivedfeln= ien sláttern, uno, fperrigen; in Den Blattadfeln fregenden 3weigen. STlumen ebenfalls in Den Blattadfern, Die 3roitterblitten mit finf: Tapviger Blumengúlle, 5 Staubfáden und cinem Jrudtenoten mit gabeliger Tiarbe; Die meibliden mit zmeien, fduppenformigen, oft

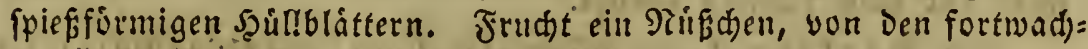
fenoèn Scullablattern umgeben, Segre gemein ift A. patulum, mit gegeniberffereenden, oreicçig lanzettformigen, faft fpiefartigen, ge= zógntent Biátern und weit abftchenden 3meigen. Şúllblátter auf

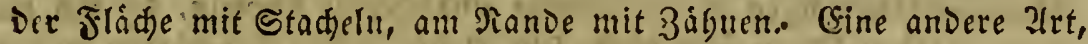
Die Ga artenmelde (A. hortense), mit Dreieckigen, gejáfnten, gleid)=

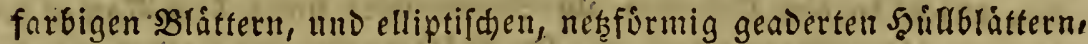
wito in B̈arten angebautet uno afs Bsemúfe gegefien.

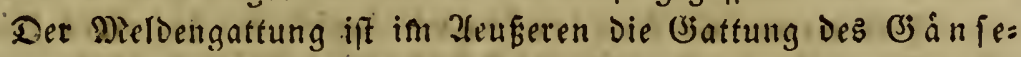

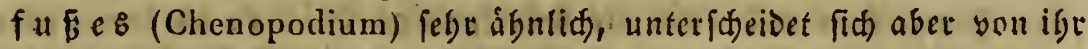
Durd) ftets zmittrige situmen, uno Den mit Der Frudte fid nidyt ver= suvernoen, füflappigen Sield. Sei \& in ne ftegt fie Daber in ber fiunfte $\mathfrak{n}$ Si f f f e:

Bierunojmanjigfe Siralle. Cryptogamia.

\$. 163. Der Eharafter biefer Yeğten Nlaffe bes $\mathfrak{L}$ inne $i=$ (d) en Syftemes befteht in bem Mangel aller beutlichen $\mathfrak{B l u t}=$ men unt ber in ifnen vorkandenen Srgane. Prichts befto we= niger bilben alle Samen, bie fich aber baburd yon ben Sa=

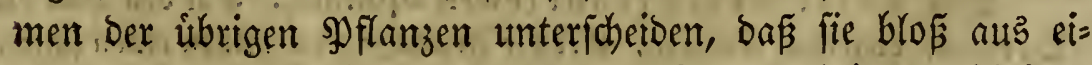
nem einfachen $B$ Kảzchen beftehen, wáhreno bei ben itbrigen (Fimeis, Seim und Samenlappen fich) untericheiben laffen. Weit arjo diefen Pflanzen ber Samenlappen felylt, beipen fie 
auch 2lotylebonen, uno ihre Samen ridtiger Seims Érner (spori): Sie liegen felten frei in ber Sflanze, mei= ftens innerbalb gróṕerer Sáfé (sporangia), ober fórmlicher Rapjeln. Rach Rinne jerfällt bie Slafle in 6 Dronungen:

1. Jam. Jarrengrater (Filices). Pfanzen mit Deutlidgen, zum Theil gegliederten, róbrigen, allermeift gefiedertert oder fieber: fpaltigen Blättern, und Sieimérurnern in Siapfelli, welde bauferweis bei einander ftelfen, und in Der Diegel an Der unteren FTádte Des

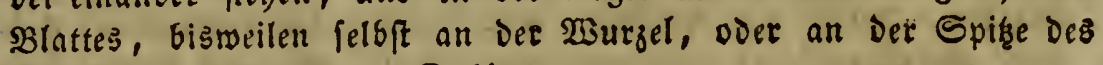
Etengels befeftigt fint. Dabin:

Der 5 a d telf al $m$ (Equisetum palustre), mit grade aufred)= tem, Goblem, gegliedertem Stengel und quirfformig geftellten, Dinn= nen, gegliederten, róbrlgen, wie Der Stengel gefurditten, fünfectigen

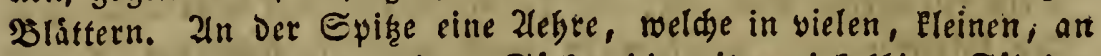
geffielten Sdeibden befeffigten Sácken Die mit zroi kolbigen Fádaen

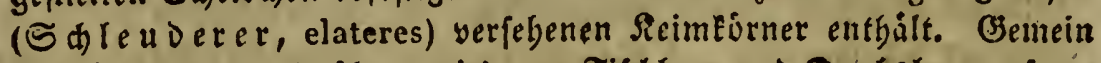
in Eimpfen uno (3räben, wird von Tildlern und Dredgslern gefams melt uno bum goliten benuţt.

Der $\mathfrak{Z} \mathfrak{a} \mathfrak{r}$ la pp (Lycopodium clavatum), eine Eleine Pflange mit

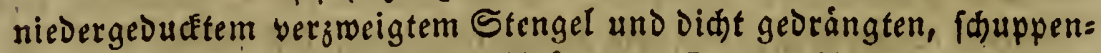
formigen, Eleinen lanjettliden Bláttern. In Den 2ld fẹln Der leşten

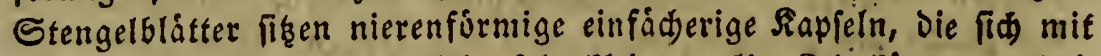
einer Silappe iffnen uno viele, fegr Eleine, gelbe SieimËurnet entgal: ten, welde in ben Sfficinen als Streupulver unter beni samen Des

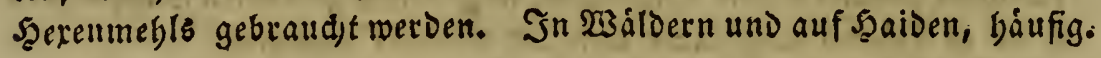

Das gemeine Jarreneraut (Aspidium) bat einen Eriedens

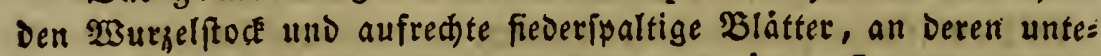
$\mathfrak{r e r}$ Seite Eleine runve mit einem Silige umgurtete Sapfeln baufen: weis zerfiteut fiegen. Seder Siapferbaufen (sorus) yon einer binnent \$aaut (Sø)leier, indusium) bedeckt. A. filix mas bat cinen runben Sdleier, 2mal gefiedertes Eaub, Deffen Fiederlappen lärglid, aber ftumpf, und am siande mit ftumpfen 3ábnen verfegen find. Jrudft= baufen neben Der TRitterrippe, Diefe uno Der $\mathfrak{B l a t t f f i e l}$ mit braunen, troćnen Spreubláttern. - A. filix femina bat länglidje Salleier, fpis Dreifad) geságnte Fiederlappen und lánglide Frudthaufen, seide fegre gemein in unferen Báldern.

Das 2lorerfarrenEraut (Pteris aquilina). Shat beri baa des Dorigen, Dieferben Sapfeln, aber Die Jrudthaufen bilden einen fdima= Pen, am Mande fortlaufenden Strid, mit binnem, nadh Jnnen offe= nem Shleier. Das Dlatt ift boppelt gefiedert, und de Eappen find, beforbors am untern Tgeile, in länglide ftumpfe 3igne getbeilt. Dev 


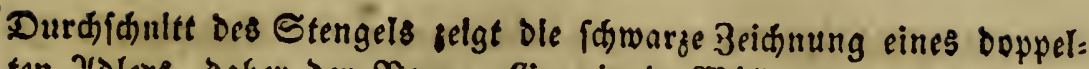
ten 2lolers, Dnber Der Nante. Bemein in æaáloern.

2. Fam. $\mathscr{E} a$ u $b$ m 0 ofe (Musci trondosi). Sileine Pflanzen mif Dinnen, versmeigten Etengeln, moran kleine, fdimale, zugefpişte,

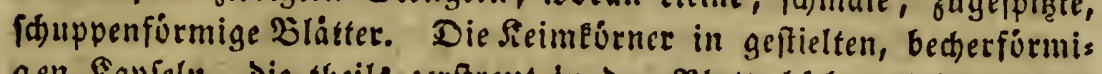
gen. Sappern, Die theils, zerftreut in Den Blattadfern, theils an Der Episze der Stengel fteben. Seve gapfel yon cinem Dectel verfकlof: fen, und allermeift am Diande mit 4, 8, 16, 32 ooer 64 3ágnen, fos wohl in einfader, als aud Doppelter Pieibe, verfegen. Die Rapfel bevecft, fo lange fie gefdloffen ift, eine bäutige, Dúnne glübze. Man Eenut fegre viele 21 rten, $8 . \mathfrak{Z}$.

Das Torfmoos (Sphagnum latifolium). Rapfelnuinoung

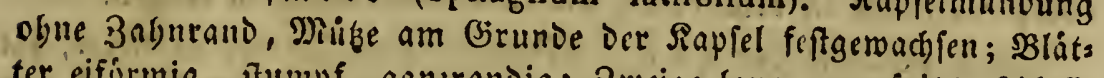
ter eifórmig, fumpf, ganzrandig; 3weige lang, bugépilst, follafi. Sn Eimpfin uno Torfmooren gemein.

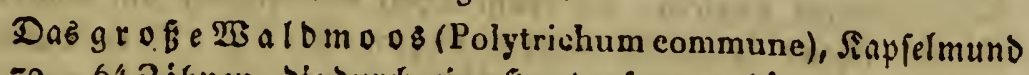
mit $52-643 \dot{a} \not n e n$, bie Durd eine Şaut bufammenjä́ngen. Stengel aufred)t, ungetbeilt, Biătter. biemlidi abifthend, am Diande uno, auf

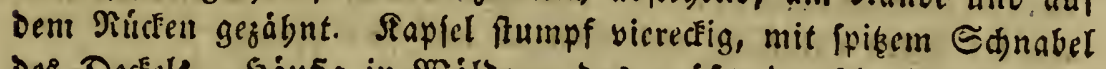

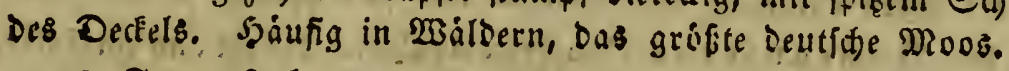

3. Fall.. Ee berm o ofe (Musci hepatici). Eie baben Die Sienn: zeidjen ber Borigen, aber bie Sapfel beftegt aus mebreren (2 ober 4) Silappen und bat Eeinen Deçel. Das \&aub ift Dímner, breiter; bib:

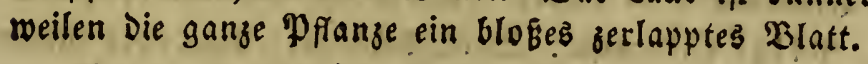

Die bierber geborigen battungen fint unmidtig und eben nifft felgr: Gáufig, Daber mir Feine namentlid anfübren E⿱㇒nuten.

4. Fam. Fle d te $\mathfrak{n}$ (Lichenes). Dlefe prlangen haben gar lei= nen Etengel mebr, fonbern beftegen blof alls einem fladen, aim

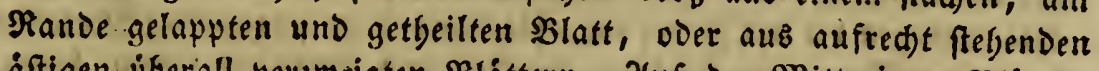

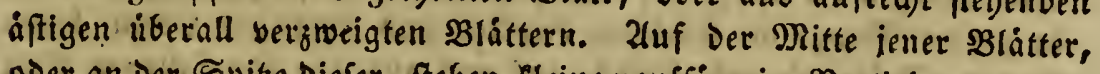
oder an Der @pilze Diefer, fteben Aleine napfförmige Bertiefungen ober

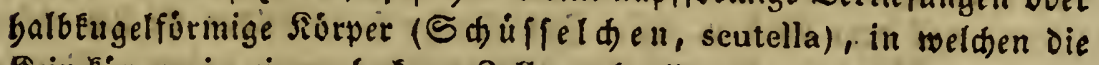
Sieimformer in einem lodferen Bellgewebe liegen. Die Jiedten wadss fen theirs an Baumftimmen, theils auf nadten Jelfen, felten auf Der ErDe, und finden fid befonders in Ealten Niegionen, Daber bet uns in Bebirgsoggenden. Mande enthaltent nabrjafte Stuffe fúr Renfden und Thjiere, $\mathfrak{z}$. $\mathfrak{b}$.

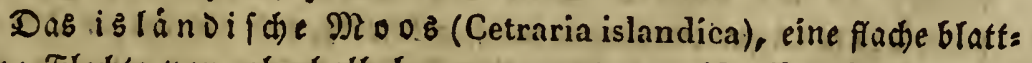
artige Fledte yon oberbalb brauner, unten weifigelber sarbe, bei welder die. Sdúffelden auf Der Ditte ftegen. Sie wádift befonders

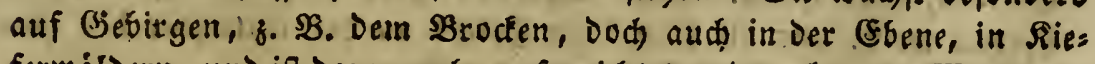
ferwáldern, und ift Dann megr aufgeridtet unb. zerlappt, 2Ran wens 
Det Das ierảndifde Doos wegen feinex nabrgaften Beftandrbeile gez gen Drufteranlgeiten mit Erfolg an.

Das Renntbiermoos (Coenomyce rangiferina) ifte eine aufs redte, vielfadh getbeilte bijfhelformige Fledte, mit balbEugeligen, gefárbten Schiffelden an Enve. Die Flechte jelbft hat cine weif: lid graue Jarbe und braune Sdiffelden; fie findet fid uberall auf Sacioen und in fiefermaloungen an DeE Eroe. Die Renntyiere tábs ren fid befonders von biefer und verwanden 24rten.

5. Tam. 2lgen (Algae). Wlatts poer robrenformige, ver: zweigte ober einfache $\mathfrak{B a f f e r g e r w a d j e - v o n ~ g r u h e r , ~ b r a u n e r ~ o v e r ~ r o t j e r ~}$ Farbe, welde ibre Sieimérner theile in ben 3ellen Der Blatter over Rógucn, theils in befonderen, blafenformigen Fapleln tragen.

Die Ojattung Der $\mathfrak{z}$ a $n g e$ (Fucus) findet fich nur im Meere, bat cinen verzweigten, mit leverartigen Blättern befef̧ten Etenget und blajenformige Siapleln, Die zum Theil ummlttelbar am Rande' Der Blatter fiţen; ;o f. $\mathfrak{B}$. beim $\mathfrak{D l}$ a fen $=\mathfrak{T}$ ang (F. vesiculosus), welder an Den nordoeutichen Sfiften bánfig vorkommt, uno aus langen, jiemliab breiten, lanjettformigen, bráunliden Dláttern beftebt. Znoere Zirten entfalten viel Shleim und find efbar.

Dic $\mathfrak{B}$ a d c onferve (Conferva rivularis) befteht aus einfaden, Dunnen, graden, febr langen Fäben, die gegliedert find. Fedes Gilied Dreimal fo lang aỉs breit, inwendig Feimérner entbaltent. Farbe Dunkelgrún. Flockenmeis in Elaren. Báden; an Steillen befeftigt:

6. Fam. Pilze (Mycetes): Didé, fleifahige, jellige, ober bunne, fadenformige, niemaís grin, wobl aber fdwarz, blau, votg, gelb odec braun gefárbte Siorper; Deren feimlorner thcils frei, thcils in bi= fonderen $Є$ hlauden eingefoloffen, Durh die gange Maffe, oder nuf Det Sberflade in cincr bejonderen ङ bicht (hymenium) abgrtagertfino. Sie entfteben bei Der Berfesung thierifder oder pflanglider Diaterien in grofer Menge an feudten, DunElen und fdattigen Drten, befon= Ders in Frubjabr uno \$gerbft, auf uno aus ben abgefallenen sblit: tern, auf Dem . Rift u. Ogl. Su Sibaffer felbft biroen fie fid nidf, nue an Fiorpern, Die mit Der \&uft in Beruigrung ftẹels. gliande Pifge fino effor, andere Dagegen fino giftig uno bianft gefábrlich.

Einige, wie bie. Sđ wa a m me (Fungi), baben einen Stiel utid

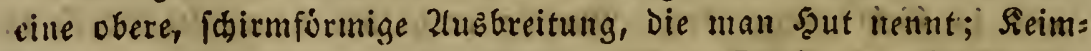
Eirner an Der unteren Seite Des Şutb. Dabin Der Jliegen=

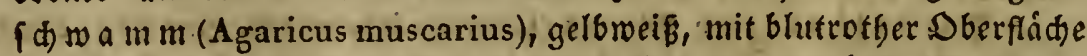
Des Şuts; Die untere in ftraglige Falten gelegt. Şâufig in Siefer= wálsern, ‘fber giffig. Der Cbampignon (A. campestris) ift ifgerall meif, uru bie ftrabligen Jalten an Der Unterfeite Des Şuts find violetbraun. 2luf Biebreiden; ęfar... 


\section{Bierundzranzigfte Slaffe. Cryptogamia. \$pilje. 161}

Der Fe uer fdr a $\mathrm{mm}$ (Boletus fomentarius) Jat Eritien Stiel,

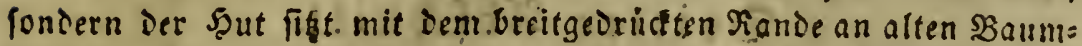
ftammen, befonders an 2tpfelbaumen, feft. Unterfeite bez 5 Juts feiu Durdischerf; Jarbe gelbbraun. Wird bur Bereitung Des Feuer. 何rammes betrubst.

Invere beftegen ats Eugêfformigen Daflen, in berew innerem

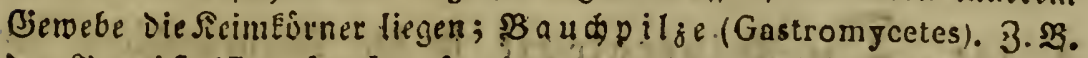
Der Dovift (Lycoberdon hovista), von Der Briffe pines Şúfner: ries utuo oruber, mit zugefpitgter 23 Brgelfeite; Jarbo mildweiß. 2luf Siebweiven, platst reif obeh in Der Ditte auf und láft die vielen, braunen Reimliorner mie Etaub berausfprúben. - Zud die çbare T: iffel (Tuber obtestum) bat biefe Form uno \&bnjiden \$au. Sie findet fid tief in Der Erice an olten \$Bummurieln.

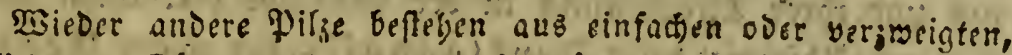

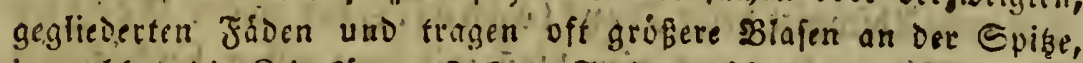
in rocthen die Reimesrner fteden; Jabenpilge. (Hyphomicetes), b. B. Der $\subseteq$ dilm mel (Mucor) alf eingemadten Eadien.

Tod andere enifid befiegen ans einem bloben Pulver vieler unenolid Eleiner fielmkirner, weldes fid unter Der Dberbaut leben= Der Planjen, die aber Daourn, menigftens an biefer Etelle, getid: tet meroen, bildet; 5 taubpllze (Coniomycetes). Diefer 2 rt ift

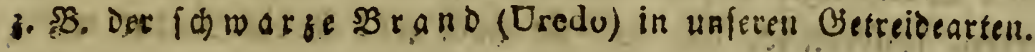




\section{Dritter 26fGnitt. \\ $\mathfrak{D} \mathfrak{i} \mathfrak{n} \in \mathfrak{r} \mathfrak{a} \mathfrak{l} \mathfrak{D} \mathfrak{g} \mathfrak{i}$.}

§. 164. Die Mineralogie ift bie Raturgefdidte ber anorganifdén (\$.2.) Raturfórper ober Mineralien.

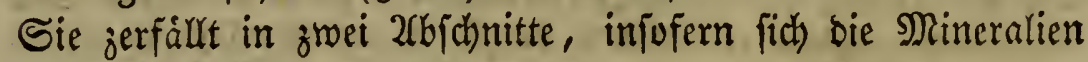
theils nach ifren Hebereinftimmungen und Serjobjedenheiten im

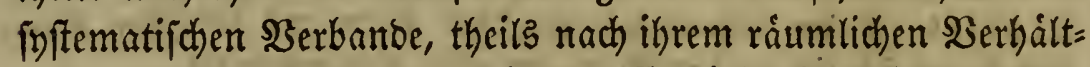

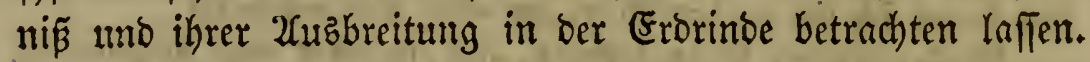

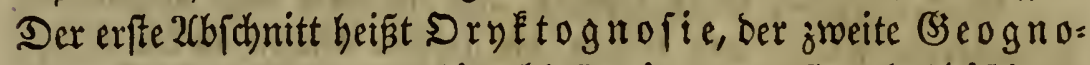

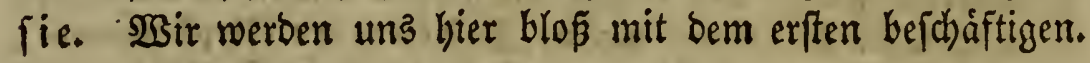

\section{Divetognofie.}

§. 165. Da bie anorganifhen Ilaturférper Éeiner bejon= beren $\mathfrak{B e r F}$ zeuge, behufb ibreb Entftehens uno Beftehens, be= burfen, fondern fo, wie wir fie noch jekt in ber Patur wahrs nelymen, auz ber Şand bez Sdjopfers herborgingen, fo fón=

- nen in einer vorláufigen Schildecung nur bie Şaupteigenichaf= ten mit ifren Berfobiebentheiten betract)tet werben.

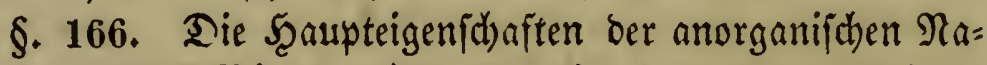
turéorper, ober Mineralien, fino biefelben aller naturticten Siorper, uno bezieben fith auf:

a) Die (se ft alt berjelben, ober bie áußeren Utmrifie, un= ter weldten fie in ber Ratur ericheinen.

b) Die $3 \mathfrak{u}\lceil a m m e n j e\{u n g$, ober bas (B)efúge ihres snneren, bas fich oft fcton an ber áußeren sberfláche, immer aber auf ber $\mathfrak{B r u c h f l i c h}$ e, verráth.

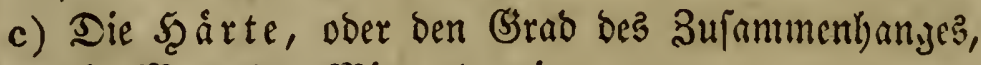
welchen bie Marfe bes Minerals jeigt.

d) Die Schwere, ober bie Siraft, mit welcher es auf feine Unterlage brưdét.

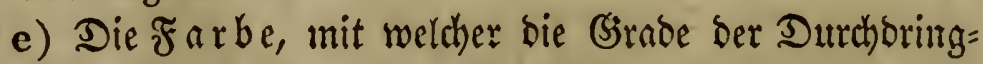

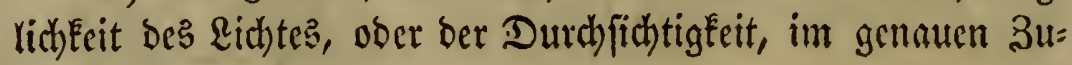
fammentyange ftelen. 
f) Die $\mathfrak{R} \mathbf{a} f t e$, weldbe bie Mineralien noch fonft befi= ken, z. B. magnetif(h) ober eleftrifhe. Diefe (Eigenichaften beisen phyjik alifde.

g) Die Beft andtheile, aus weldhen bie Mineralien jufammengejest find. Dieje unterjutht bie Ehemie ober Scheibekunft, baher man foldhe (Eigenfthaften aud mit bem Namen ber demifd en belegt.

§. 16\%. 2ure bie genannten Shaupteigenichaften ber Mi= neralient mit inren verjobiebenen $2 \mathfrak{b}$ ftufungen im 3 uammen=

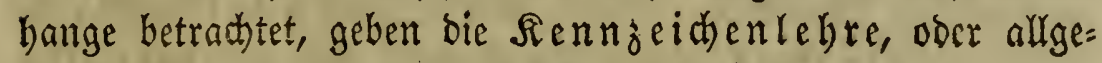
meine snineralogie, mit weldher wir beginnen.

\section{Siennzeidenlebre.}

\section{A. Son ber (sieftalt.}

\$. 168. Die (Seftalt ber Mineralien ift theils beftimmt, theils unbeft im $\mathrm{mt}$.

Die beftimmte (Bjeftalt, b. b. biejenige, weld)e bei al= len Inoivibuen eines Minerals fich gleid) bleibt, heift audb bie Eryftallinifde. Stryftallinifhe Sórper, ober. STys it alle, fino alle (ymmetrif won Flachen, weldhe in Janten

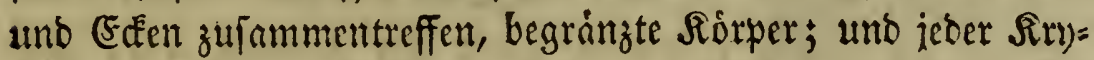
ftall cines Minerals ift ein eben fo wahres Sttbivibuum, wie bie eimzernen spflanjen und ghicre, auz welchen bie 2 rtten in Der BotaniE uno Soologie beftehen.

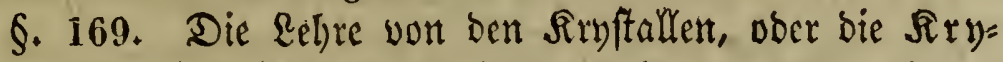
ftallographie, ift ber wichtigfte sheil ber infematijhen Mineralogie, weil wir ohne Senntni范 berfelben bie Mineralien nicht unterfheiben fonnen, baher hier einige allgemeine (5igen= finaften ber Siryftalle anjufiugren find.

Seder Siryftall wirb von $\mathfrak{F l}$ achen, allermeift ebenen, mitunter gewólbten, begrángt. $\mathfrak{B S O}_{0}$ bieje Fläd)en aneinan= Der ftoß̌en, entfelyen bie Sinten, und mo Ranten zufam= mentreffen, bilden fid) (5) df en. Einte grabe sinie, welche zmei gegeniberftelgende Edén, Santen ober Fláchen verbindet, heiṕt 2ud $\mathfrak{e}_{\text {. }}$ 
'Rach dex 2lnzahl und verháltniß̄mápigen Srópe ber in ben

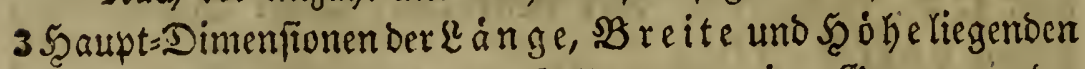
xhien laffen fich alle siryftalle unter vier (Siruppen oder Srftente bringen.

1. Das icfferale Syftem hat orei gleiche Xabjen, baber audh gleidbachfige genannt, welche fid) rechtwintig burchichnetion.

2. Sas pyramioale Syftem hat ebenfalls orei fids rechtwintlig fhneibenbe 2 hjen, von welchen z wei gleich, bie oritte $g r$ ó Bex ober Eleiner ift; es mirb auch bas vierglis orige Syftem genamt.

3. S)as prismatifche Syftem; bie brei fich rect)ts wintlich jomeibenben Xoblen haben alfe verfhiedene länge, bas ber babein= uno einadifige ober ungleid a dh fige Syftem.

4. Das rhomboebrifde Syftem bat vier 2(d)fen, brei gleiche liegen in einer (5bene und faneiden fich unter SBinfeln von $60^{\circ}$, die britte grofere ober Fleinere fteht fent= recht auf Dem Surchidnittspunfte; beift auch Das ore $i=$ uno einadige Syftem.

Bon ben 2(d)fen heist die ungleiche Scauptach fe, und bie figur, weldie entffet, wenn man cincn. Sdynitt in ber (Ebene ber anberen Xajen ourd den Neryftall legt, beipt

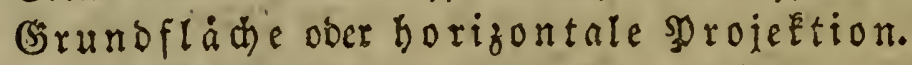

Denft man fich die Enopunfte ber 2(d)ien cimes Syftemes Durch Sinien verbunben, fo entftebt Der Ssrunderyftall, aus wel= chem fid alle anderen ableiten laffen; und zwar theils burch)

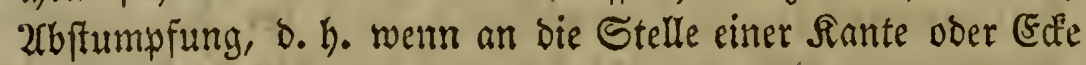

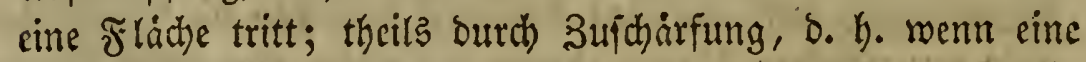

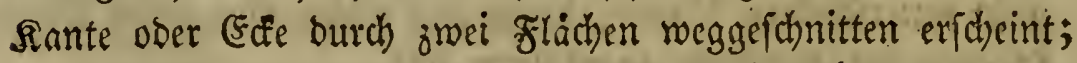
theils burch 3ujpikung, wenn baffelbe burch brei ober mebrele Flaben bei ber (F)e geidjiebt; theils endlich, wenn einzelne Flas chen verfhminben, andere Dafur machfen. Trifft bies bic balbe anjahl, jo heiät ber neue Sryftall cin halbirter oder bo mie. orifder, ber uripringliche im (siegenfak homoebrifa.

Die Sirnftalle meroen nad) Der 2(nzahl ber Fladben be nannt. Die Eleinfte 2unzabl ift vier, ein foldher stryftall 


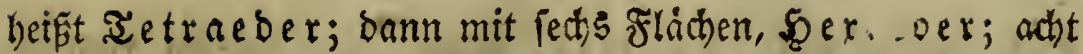
Flächen, DEtaeder; zwolf Fláchen, DobeEaeder; zronzig Flächen, Skof aeber; vierunbzwanjig flád)en, Jevofitetra

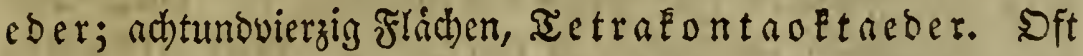
haben verfhiebene Sirgftalle gleiche Fladienzabl, bann unter fdeibet man fie nach ber form ber Fläd)en. So giebt es Iriangularbobekaeder, b. h. 3wólflichner, deren Flás d)en Dreiecte find; Rhomboibaldodefaeder, diefelben

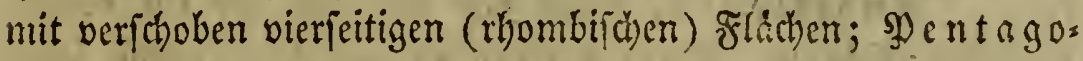

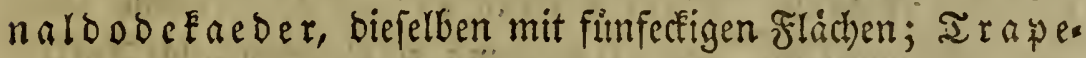
zoeber, roenn bie flichen vieredig aber unregelmårig fino. Dolyeber beist jeber firnftall mit brei gleid)en 2echfen. Dyramibe heist ein von Dreiecten begrinzter Aityftall, bei weldhem fid alle gleichen Spitsen in ben (Enopinten ber

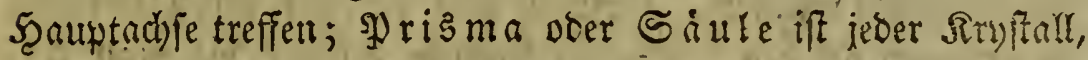
beffen Santen ber Scauptachje parallet lanfen; I afel beipst er, renn bie SJauptadbje fehe Furz uno am (Ente ourch eine breite Fläche abgeftumpft iff.

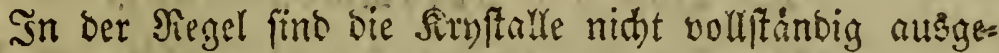

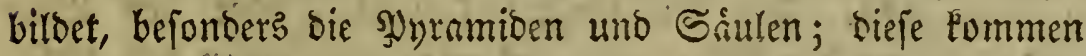
meiftens in "Sserbinoung por, fo baf eine Snramibe am Ente ber Saitle entffelt, willyeno der firyftall mit bem anderen

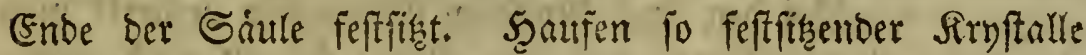
nennt man eine D ruje.

§. 170. 3ur unbeftimmten (Geftalt rechnet man alle nicht Errfftallinijhen Formen; Dergleichen fino bie folgenden.

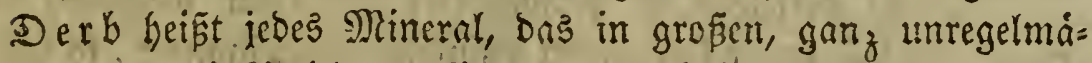
figen, ia millfürtichen, ectigen, oft zufáltig entfantenen Stü.

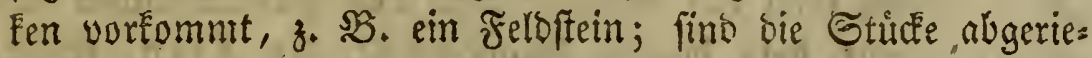

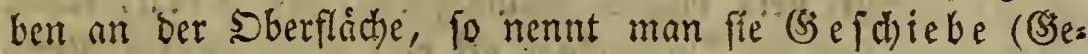
rolle). (Fingelprengt, ein Mineral, bas als Eleinere Studfe ober אiorner, bie in anderen Foffitien liegen, vorkommt. Si $\mathfrak{i}=$ nig, menn bieje Sticke frei find, nicht in einem sindemittel

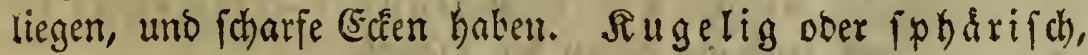
weun bie frorm Eugelnunb ift. Rierenformig, wenn bns S) 
flache form hat. Rnollig, wenn unregelmápig runolich unt mit (Erbabenheiten auf ber Sberfliche. Iropffe infor mig ober ftalaftifch, wenn bas Mineral wie burch 2 fuf $=$

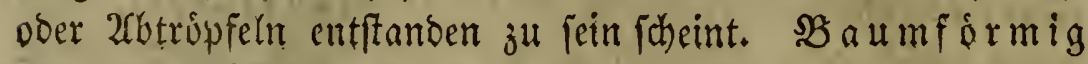
ober bendritifch, wenn es fich wie ein 3 weig mit Eleinen Blittern, z. BB. wie ein Moos, verbreitet. (S) eftrict, wenn feine nabelformige Fiorper ein regelmásigez Minfohenwerk bil= ben. - Ungeflogen, wenn das Mineral als ein binner

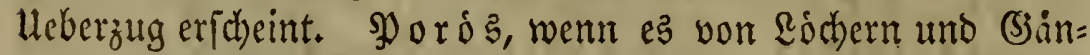

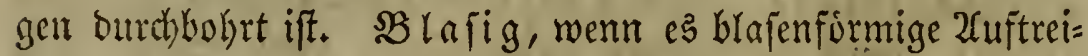
bungen bilbet, ober runbe, bohle Raume zerftreut in feinem

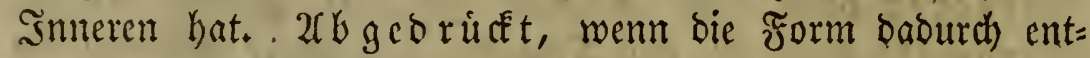

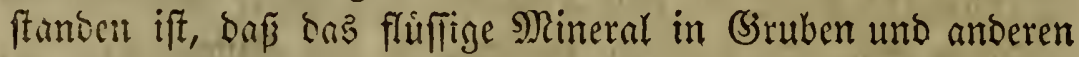

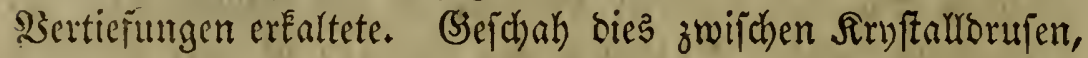
io entfteken oft den Siryftalfen áhnliche Sidrper, bie man $\mathfrak{X} f=$ tereryft alle zu nennen pflegt.

\section{B. Son ber 3 afammenfekng.}

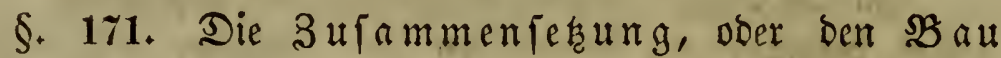
bes smneren ber Mineralien, erkennen wir, went mir biejel=

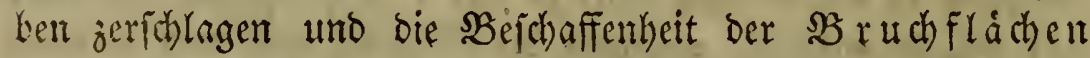
betrachten. Bei ben Eryftallinifden Mineralien bemerft man bei biefer Unterjud)ung, baß̧ ihre Maffe uberall nach unver= inderlich) grabflachig parallelen sheilungsebenen zeriprengbar

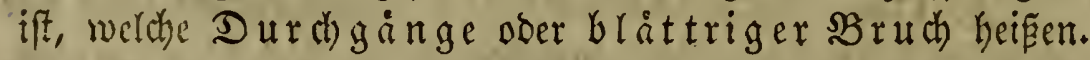
Die 2(njahl Derfelben ift veribiteden; find ihrer mehrere, fo

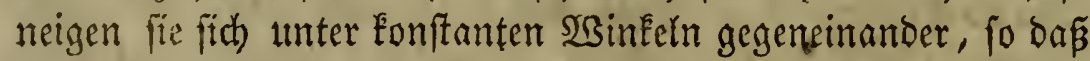
man, wenn man bas Mineral nach ben \$arallelflichen Der= Felken trennt, ftets biejelbe (Srundgeftalt, ben Rern, erhält. liebrigens fimb bie Durchgånge oft von verichiebenem \$3erth, injofern fich bas Mineral nach bem einen bann leichter thei= len lipist, als nach ben anderen. Derienige, in welchem bie

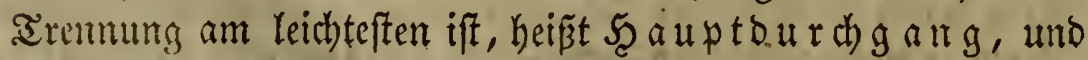
gel)t meiftens jenfrecht gegen bie Scauptachje.

Bei den unEryftallinifchen, ia auch zum aheil bei Eryftalli=

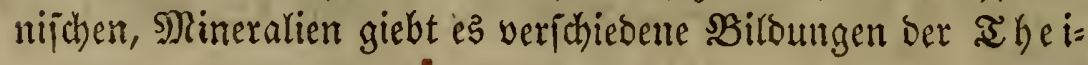




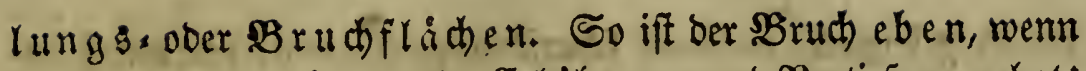
bie $\mathfrak{B r u c h}$ fliche feine großen (Frhỏhungen und \$ertiefungen hat; uneben, wenn regelmápige (Erbjhumgen und Sertiefungen ba

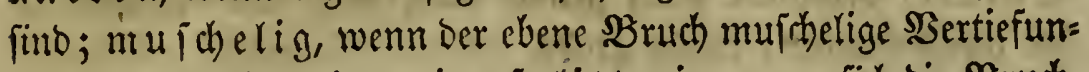

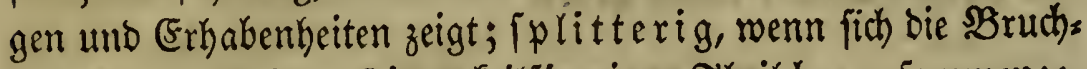
fláche als alls vielen feinen feilförmigen aheildten zulammenge= fefst jeigt; hafig, mit noch fleineren von ber SBruchflache lob= geriflenen aheilen, bie eine umgebogene Spibe baben.

Bei vielen Mineralien bemerlt man außer ben genann= ten 2(rten ber 3ujammenfefiung nod eime britte, welche man mit bem Ramen ber 2 b fonberung bezeidhnet, und barun= ter eite urfwringliche fdo on vorhandene \$rennung in beftimmt geformte Stude verfegt. Nidjts befto weniger hängen biefe Eticke genau zufammen und lafien fid) nur gewaltiam tren= nen. Die 2lbjonberung ift nun theils fryftallinifch, wenn bie gulammengefingten \$artifelchen fleine Fryfforle find; theils

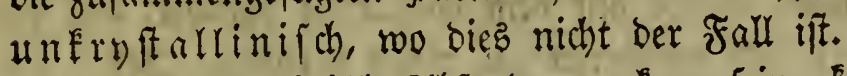

Die fryftallinifche 2(bjonderung Eann fein: Fó rnig, wenn bie 2(bienberungsfticle ziemlich gleithe Dimenfionen baben, alfo nethr weniger Eugelformig ober polyebrifa fino; for a $a=$ lig, wenn die 2lbfonoerumgsftucte in ben Dimenfionen ber Lánge uno sisreite bebeuteno, ber Diffe aber unbebeuteno, alfo platten = ober tafelformig, find; ftảngelig, wenn bie 2rbionderungsftude bie Dimenitonen ber Länge vorwalteno cutwicke!t zeigen, aljo prismatijh), theils mit, theils on ne spy= remibenflichen am (Ende, gebilbet find. (Geken diefe sprismen wie von einem Mittelpunkte aub, fo nennt man bie $26 b$ jon = berung ftrahlig (Strahlfiȩ); im Uebrigen laufen bie Flei= nen Siryftalle parallel oder bunt burch cinander (2frjeniffieb). Die unfryftallinifhe 2rbjonderung zeigt bieperben brei Szautformen, bod immer nit bem wejentlichen Unterichicbe tes Minngets wabrer Sirnffallifation an ben eimgetnen sheilen. ß3ei Der É linjenformig, oattelformig, edig ober erbig, wenn bie Sgartifclchen fehr flein, ftaubig find, und fich leicht ablo= fen; bei ber folsanligen, ober fohieferigen, find ces flache, oft 
etwas gebogene, meiftens foharffantige \$ुlatken, bei ber ft à n. geligen fino fie bald grabe, fantig uno abmen (o. BB. beim

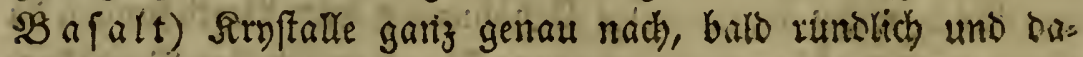
bei theils Erumm, theils grade.

\section{Non Dè Şárte:}

8. 172. Die (Eigentid)aften ber Mineralien, melche fich auf bie Cobåfion beziehen, pflegt nan gemeiniglid) nit bem 2usorud Der Scarte=(Figenjd)aften zu belegen. SBerner un= terichico füf Urten: bart, was am Stahle frunten gicbt; balbhart, was vom Neffer Einoride ainimmt; weidj, was mit Dem Meffer leicht getheilt wirb; felt r weich, was bem Srud des fingernagels nadgiebt; und jerreivlidf, ivas bei bem blopen sisifhen mit bem finger fich abreibt. In neueter Zeit bat Nobs eine genaute Skala ber Sgerte= grabe aufgeftellt, melche auf Dem Sirunda a berubet, baj ber

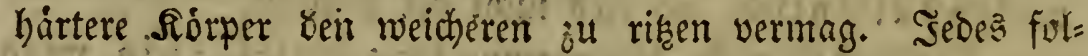

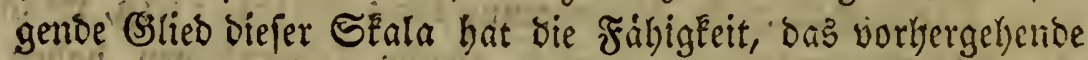

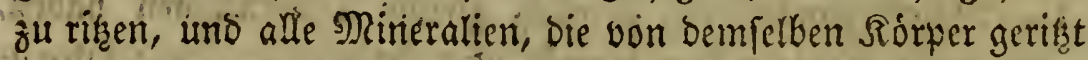
werben, haben nit bem borkergebenden gleiche Şerte, ment

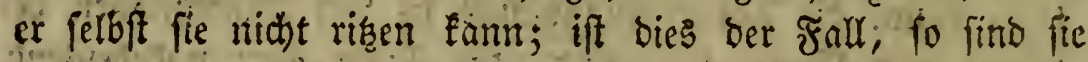
weicher. Die Esala ift folgende: 1) Ialf, 2) (šnps, 3)

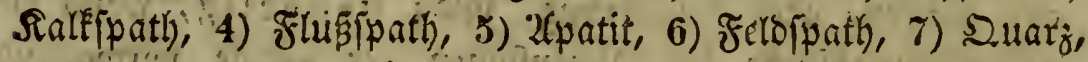
8) Sopas, 9) Saphir, 10) Diamant. Der Diamant, wel= chen fein anberer Rorper, als nur fein eigentes sjulver, an= greift, ift Der härtefte bon allen. Ser אurrge wegen bezeidynet

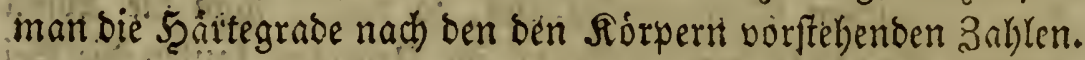

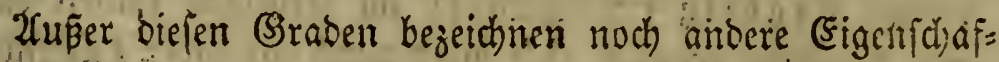
ten bie Sohafionigrabe. WBir nennen als jo!che bie folgenden: Spróbe heift ciin Mineral, bas teine Riegung vertrigt, fon=

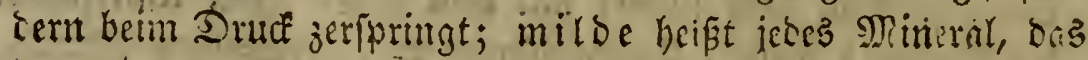
fich mit bem Neffer juncioen lafit; geidmeioig ift jes 5

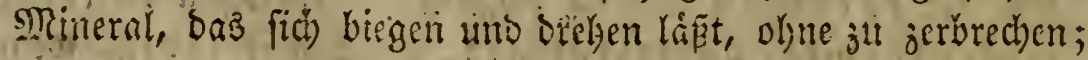
Debnbar, cin Mineral, bas fich in Dimme Drathe jieljell ind in feine sylatten fdugen läß̈t; jable, wenn die Debu= bartit for grop ift; biegfam betet eit sprineral, wab fid) 
biegen läß̈t und in ber erhaltenen form verbleibt; elaftif d) bagegen, wenn ez nach ber Biegung bie alte form wicber annimmt.

Siele Mineralian laffen fich burd) bald Gartere bald wei= chere Siorper jerreiben; bas \$Jutuer, in weiches fie bann fith auflofen, befommit ben. Miamen Strich; andere baben bie

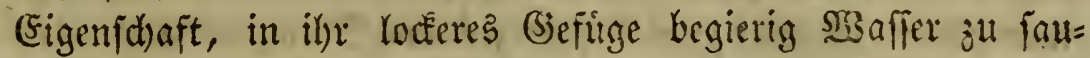
gen, Darauf bertibet bas 2 nhingen an ber 3 unge; noch andere baben eine fo cigenthumliche (Silitte, dan; fie fich wie Fett anfublen, uno Dezgrab fettig genumt weroen.

\section{Mon bar Schwere.}

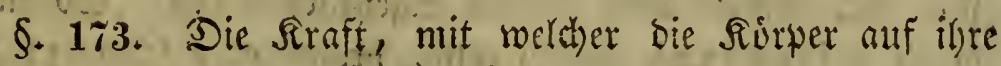

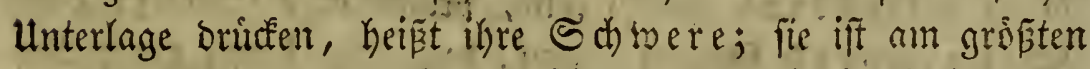
bei ben Mineralien. Man keftinmt fie auf dic $2 s e i f e, ~ b a \tilde{\beta}$ man bie Schwere eines Jorrpers ats Einfeit annimmt, uno barnach die Sabwere oer hibrigat bered)net. Dâs auf biepe

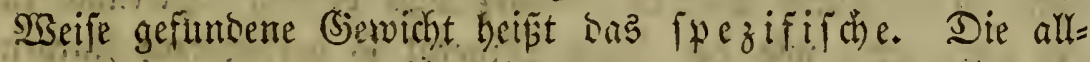
gemein angenommene (sinheit if bas Şafler, beffen (jewidht

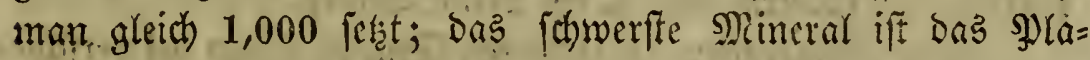

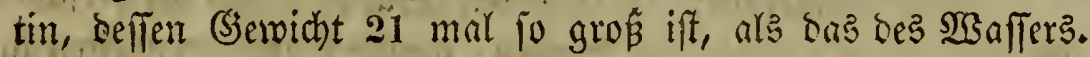
Die Methode, biefes (Sicwicht jut beftimmen, leh)rt bie \$yhylit.

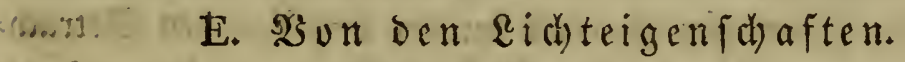

§. 174. Die Farben Der Mlineralien geboren theils bem Mitreral an jide an, uno haben in ber uriprunglicten Miichung befletben ifren (strund, theils rillen fie von frem= ben Stoffen her, bie nicht mit jum sisejen des. Minerals ge= Guven. Erfferes ift ber Fall bei ben metallifhen Mineralien, Yohteres bei ben Eroen uno Steinen.

Die Farben an fich jind veridjieden 1) nach bem (jo $r$ abe, und man unterjheibet in biejer Şinficht t ounfer, wenn bie Farbe ins Schwarje fallt; hod), wenn bie Farbe ganl rein iff; rididt, wenn fie ins şseise fállt, uno bla

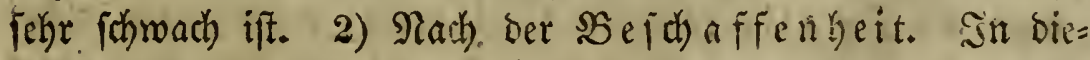
fer Scinficht untericheidet main 8 Szaltptfarben mit ilyren ver=

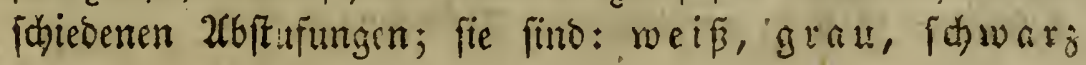


blau, grùn, gelb, roth, braun; einige berfelben fino reine, wie blau, gelb, roth, weis und fobwarz, andere ge= mif cht te, wie grau, grin und braun.

Die uripringlichen farben ber Metalle finb, als unab=

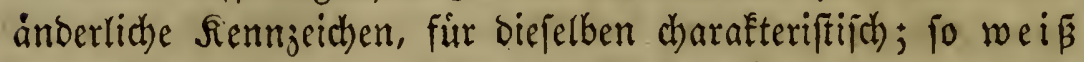
am Silber, SMlatin und Sinn, grau am SBlei und gebirge= nem Eifen, f(b) warz an Eifenerzen und Manganerzen; brau fommt bei Metallen nid)t vor; gelb am (5old und Meefling, roth am reinen Supfer, braun bei vielen Eijenerzen.

Die zufálligen Farben ber Froen uno Steine fino gróßs= tentheils burch beigemijchte metallijche Stoffe entftanden; rein find alle Eroen unto Steine eigentlich meip. So entfeltht

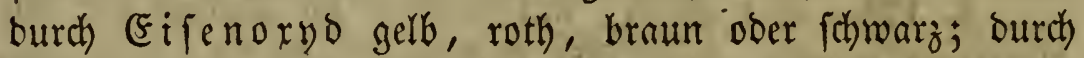
Manganoryb fchwarz, grau, roth ober blau; burch $\mathcal{S} u=$ pferoxyd blau und gruin; burd) Sobaltoxyd roth; burch (Ehromoryde grim, gelb und roth); burd) Sthwefel gelb und roth; Durd folle fdrwarz und braun. Sciernady ift

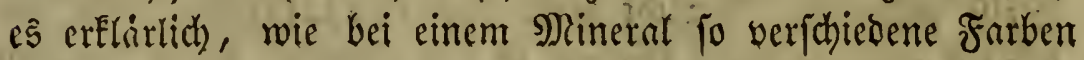
vorfommen Fomen, wie z. $\mathfrak{B}$. beim Suarz.

Siele Minernlien Der letzteren (Sruppe zeigen bei verifchie= bener 2 (nfidyt verjabedene Farben, welche \$eridbiedenkeiten in ber Brechung ober Surriefwerfung bes \&id)tes ihren (3rund baben. Entitehen biefe Farben bei ber veridhiebenen Betrach)=

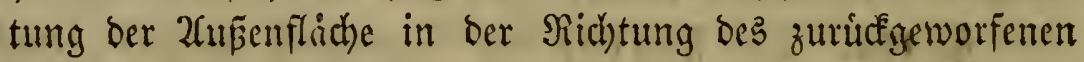
Richtes, fo bezeichnet man die Seränerlids)eeit mit bem $\mathfrak{N a}=$ men bes Farbenfpielez, wenn viele Farben jugleidh, oder ber Farbenwandung, wenn eingelne aber verjchicbene Farben nach cinander erid)eimen. (Entfelyen bagegen die ver= fchiebenen Farben bein burchgebenben Richtftrahl, fo giebt biez

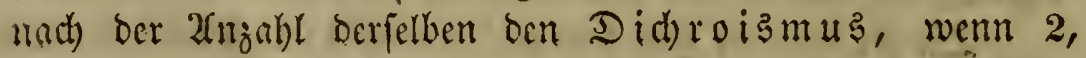
noer ben $\mathfrak{I}$ rithroismus, wenn 3 Farben fichtbar werden. פloch andere Mineraliest, bie wenig ober gar fein $\left.\Omega_{i d}\right) t$ burd)= la len; fpielen in Farben auf ber Dberfliche; biez bejeichnet man als $\$$ palifiren ober Srifiren; bei lekterem erfdei= nen alle Farben bes ఇegenbogens, bei erfterem mur cinige, bejonders blau in veridhiedenen 2lbftufungen. 
Die eigenthumliche Stâle, mit weldher Mineratien bas

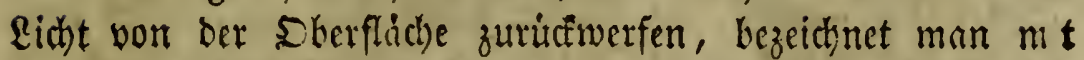

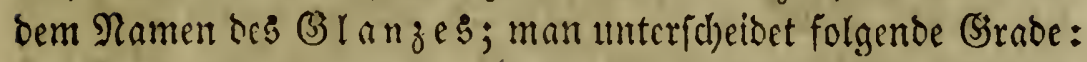
ftar glingeno, wenn ber (Silatz in betridyetlicher Entfer= nung fichtbar ift; gl\&ngeno, wenn in einiger Entfernung fichtbar; weniger glàzent, nur ganz in ber Rahe bemere bar; id immerns, wenn undeutlich glángend; matt, wenn

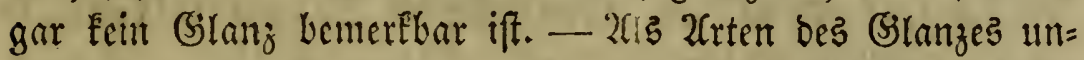
tericheibet man: Fettglanz, Sllazglanz, Demantglanj,

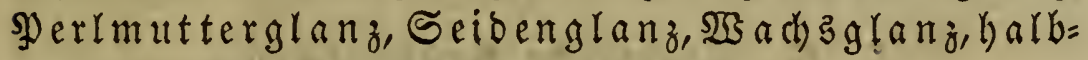
metallifder (s)lanz und Detallglang.

Die (3rabe Der Durchficthtigfeit, ober bes Berntogens ber

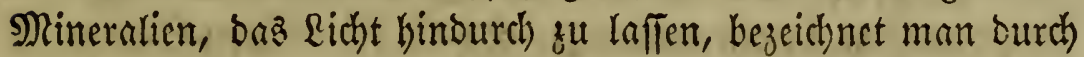
5 2aftufungen: 1) burdjichtig, wenn bas eicht vollem = men unb ungetrubt hitnourd geht; 2) halbourdh fidhtig, wenn bie (jegenftanbe hinter bem folfil getribt etfd)einen;

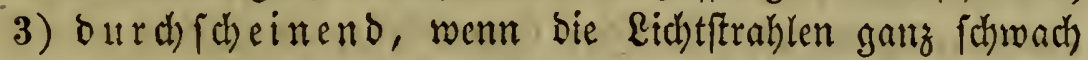
burchbringen; 4) an ben Sianten burchjcheinend; 5) $\mathfrak{u} n=$ burd $j i d$ tig, wenn Eein Ridht binourch bringt.

Zulle Sirnftalle ber brei leţten Syfteme zeigen linter fich bie (Begenftánoe boppelt, buch mit verfd)iebener Deutlicheit, eine (Figenichaft, welche man bie boppelte Strahlenbre= d) ung nennt. Sie prismatifden Siryftalle haben zwei $2($ b) $=$ fen ber boppelten Strablenbred)ung, b. h. zwei Nichtungen, in welden ber betrad)tete (siegenftano einfach erfd)eint; bie pyramibalen utho thomboedrijhen bagegen nur eine. 2(nde= ve Mineralien fangen sid)t cin uno leudyten baber eine Beit lang im Fimftern, plosplorezciren.

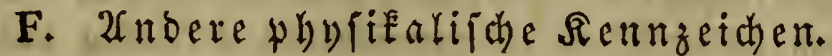

§. 175. 2fußer ben genannten, alfen Mineralien zu= fonmenten, (Figenfdhaften giebt es nodh einige Rennzeid)en, welche von untergeoroneter 2 Sidjtigkes find, und meiftens nur bei einigen Stoffen vorkommen. So nelymen alle Mineralien bie 23 ârme auf, aber fie laffen biefelbe nidbt in gleid)en (3rabe wieber fabren, leitea bie Wárme alfo verjchieben. 


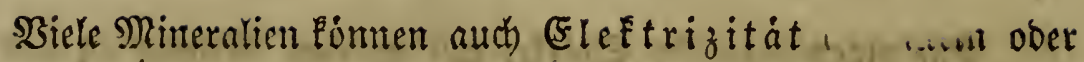
elektrif ch werben, bie meiften ourch) Reibung, einige surd Den blopen Druat, z. B. Salefpath, wicher andere burch (Er= warmen (thermoclettrifche), j., BB. Eurmalin, Borajit 1.. a. Ensfich giebt eż nod) einige Mineralien, an weldhen wir bie magnetifhe Sirritt bevbad)ten, uno die bezhalb magnetif(h) heipen. Es fint befonterz Eifenerze, weldhe biefe Eigenlithaft befiten, namentlich der Wi a gneteifenftein ober natiurliche Magnet.

\section{G. Ehemifde Senngeithen.}

§. 176. Die meiften Mincralien fino aus mekreren (Grundftoffen zufammengejest, obwobl fie umferen Sinnen als

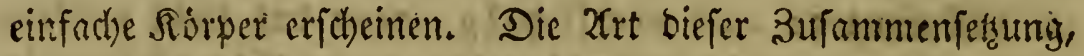
fo wie bie ङtoffe, aus welchen fie zufammengefegrt fino, lehrt bie Elyemie, baher wir bie Sennjeidyen, welche von ber Sufammenfeksung aus einfachen Grunoftoffen bergeleitet werden, che entif che nennen.

Die einfachen (Stundftoffe der Mineralien find: bic Mle= talle, die Metallotoe, Der Schmefel, ber \$ghosphor, bas Ehlor,

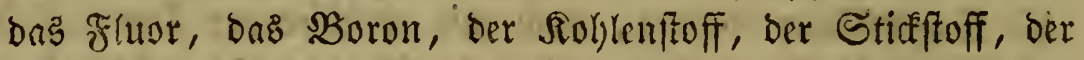
M̧afferftoff umb ber Sauerftoff. Son biefen Stoffen finden fich. nur bie Metalle, Der Schwefel uno ber Soldenftoff rein ober geoiegen; alle úbrigent gehen beftentig mit einanber, voer mit ben brei fhon genannten Stoffen, serbinoungen eist. So fino zterft die Metallotoe, welche rein ganz bas 2unjehent ber Metrlle haben, fich aber eben butrd ihre grope' Reigung, mit bem Sautrftoff Şerbinoungen einzugelen, unb burch ihr geringes fpesifiifhes (siewicht, was beftändig unter 5,0 bleibt,

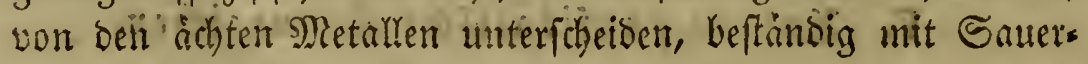
ftoff yerbumben oder oxybirt. Die sictalloibe, welche in Der Serbinoung mit Sauerfoff allcin vortommen, nennt man (Erben; die, anderen, weld)e atser dem Gauerftoff intmer

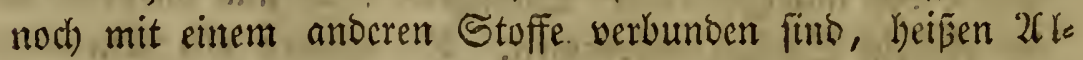
Ealien. 2llealien find: Sali, Ratron, Rithium uno 2rmmo=

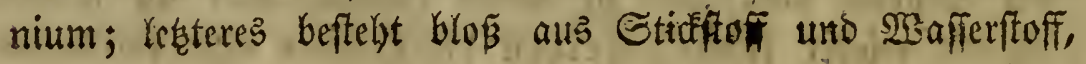


und fann nidit metallifh. bargeftellt wereen. Erben giebi

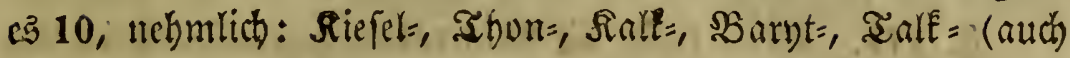

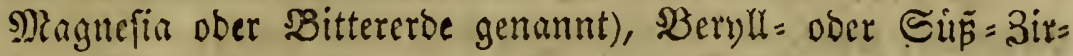

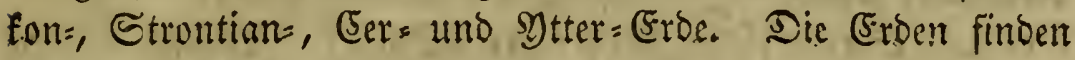
fich ubrigens ebenfalls theils unter fich, theils mit anderen Stoffen verbunden. Dieje anderen Etoffe fint sorzugsweife Säuren, b. b. Berbindungen von Sauerfor oder Misafies= ftoff nut anderen meiftens fichun orybirten Rörpern, bejonbers

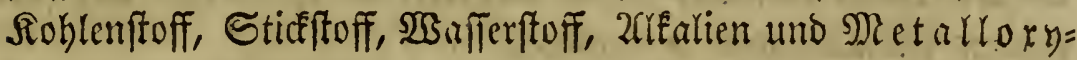
ben, b. h. Berbinoungen won Metall uno Sauertitof. 2(us ber Berbinoung oer Saure mit irgeno einem ichon orvpir= ten Rioxper entfeher bann neue Sürper, weld)e ben Ramen Salze filbren, und bie weder ben Sharafter ber Siume, als Des cinen Beftandtheiles, noch ben bes anderen Beftandthei= les, ober ber $\mathfrak{B}$ afis, wie man ily in folchen Serbindungen zu nennen pflegt, an fich tragen. Nach ber \$erfdeecenbeit

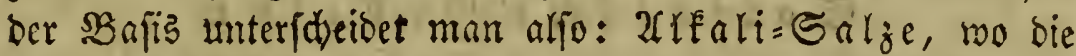
Bafis ein 2lenli ift; Froen=Salje, wo bie Bafis eine Erbe ift, und Metall=Salze, wo bie Bafis cin Metall= oxyb ift. - Endlid verbinden fidh bie Stoffe nod) gerne mit ভdymefel, am liebften bie Metalle; baraus entffelyen me=

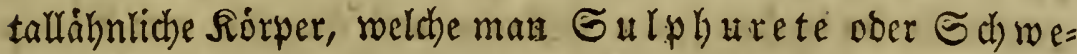
felmetarle (Riefe Blenden, Şlanze) zu nennen pflegt. 2(ud) mit dem Sauterftoff verbinbet fich ber Schwefel zut Schweferfáure, eine ber an meiften verbreiteten Sauren

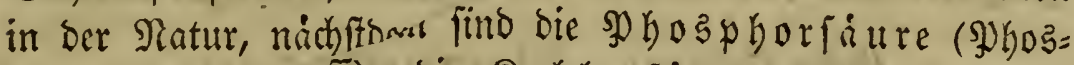
whor mit Saumioff), die Sohlenfáure (Johlenftoff und

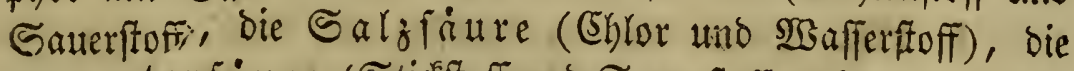

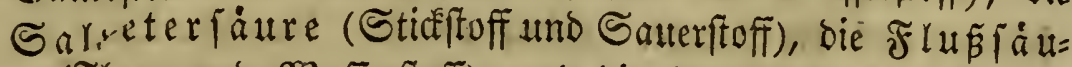
* (Fluor uno $\mathfrak{B a f l e r f t o f f ) , ~ u n o ~ b i e ~} \mathfrak{B} \cup$ raxiáure (Boron uno Sauterftofi) bie gemolgnlidyften. Metallifhe Sauren

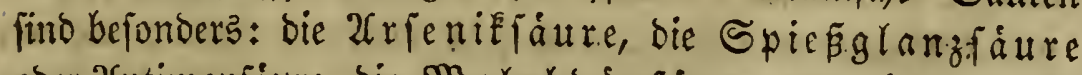

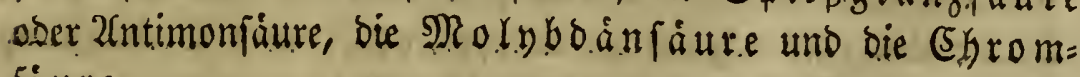
fơtre. 


\section{Syltem ber Mineralien.}

§. 177. Man theilt bie Mineralien gemeiniglich in vier (Sruppen, weld)e folgentermä̧en beftimmt werben.

1. Snlinifhe (befteine (Salze). Es fino Serbin=

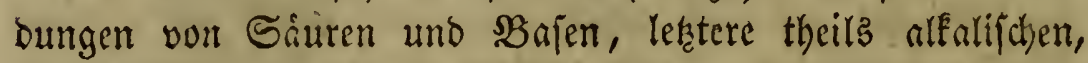
theilb eroigen, theils metallijeden uriprungs.

2. Eroige (S)efteine (Steine). Beine Erben obne Sadure, theils einfache, theils melyrere, in ber Siegel mit fäts benden Metallornden verbunden.

3. Metallifdye (sefteine (Metalle ober Erze). Me= talle allein, ober in Serbinoung mit Sauerftoff ober Edhrefel.

4. Brenglidbe (S)efteine (Brenze). Schwefel und Soklenftoff, theits rein, theils mit anderen Subjtanzen ber: mifht.

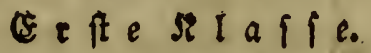

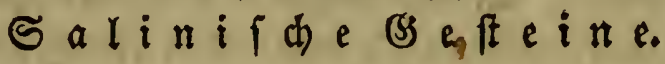

§. 178. Die meiften berfelben find burchfichtig, anbere nidts; viele zeigen bunte Farben, andere find farbenlos. Das ipez. (Semidt) wechfelt von 1,2-7,i. Die Sparte liegt zroi= fhen 1 und 6. 2Ulke find gefauerte Mineralien, meldhe vor= zugswweife im kryjtallifirten suftante auf Gjängen unb $\mathbb{E}$ agern vorfommen, in einzelnen Fällen aber auch als berbe (sebirgs:=

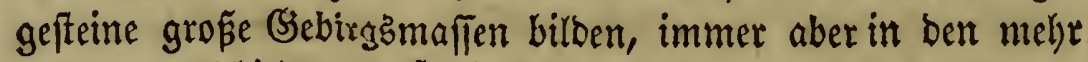
jüngeren Sdjichten unjerez E. EEorpers angetroffen werben.,

\section{Erfte Sromung. Figentliat) Salze.}

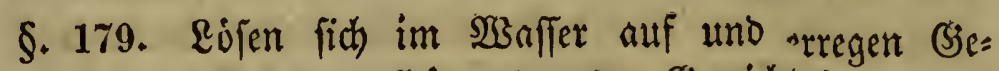
fidmade auf ber Sunge. Şäte $1-2$. (Sieroid)t $1, x-3,7$. Meiftens Eryftallifirt, ober fơnnen bods als Jiryftalle aus: inn

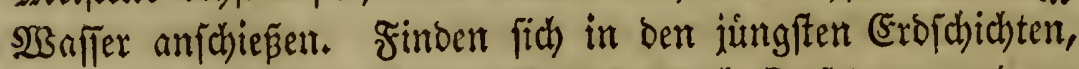

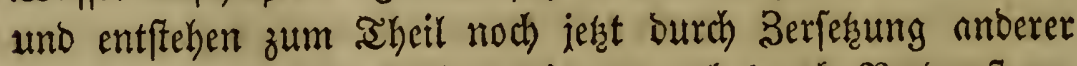
Mitneralien ober organifcher Siorper, auch burd \$erbunftung Des Suellwaffers, bas fie aufgeloft enthålt.

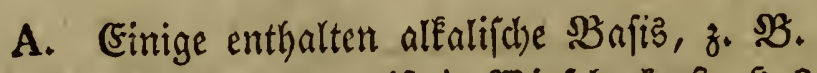

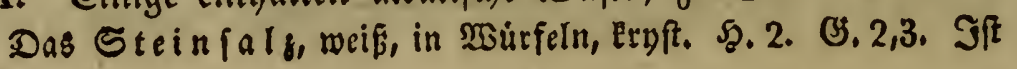


Erfte Rlaffe. Ealinifae Gefteine. Eals= Steine. 17 s

faljiaures Natron, findet fiф lagermeis im iungeren Jlögebirge, yon Tyon uno Bopps begleitet, 8. B. bei 2Bilikta; auch im Reere und in Suellen aufgelopt.

Der $S$ alpeter, weiß, wafferbell, Exyftallifirt in unregelmáfie gen fedsfeitigen Saulen. Sft folpeterfaures fiali uno finoet fid in

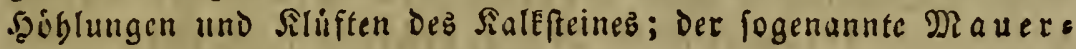
falpeter ift falpeterfaurer Siale.

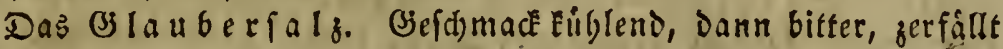

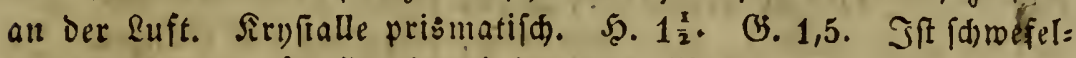
faures Satron, lift fid idwad in Ealten $3 a$ fift auf; findet fid ne= ben Steinfalk uno in Salzquellen.

B. 2noere enthalten croige $\mathfrak{B}$ afiż, z. $\mathfrak{B}$.

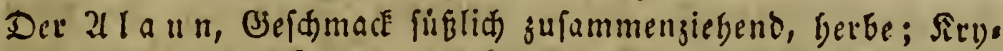

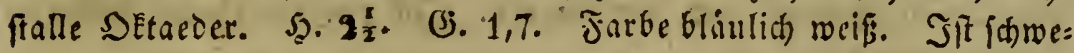
felfaure Tlonerde mit fdrefelfaurem solt. Jindet fid im Flofge= birge mit Tyon als 2llaunfhiefer (3. $\mathfrak{B}$. bei Jreienmalde).

C. Nodh andere enthalten metallifae $\mathfrak{B a j t 3}$ (Nitriole).

Eifenvitri ol, siryfalle baarformig, prismatild, Jarbe grü.

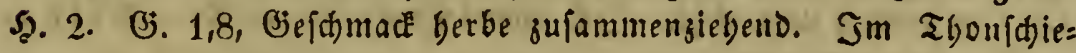
fer und Cifentgon, entfteht bei ber 3erfeţung bon હanefelfies.

siup fervitriol. Siryftalle tafelformig; Farbe blau. \$. $2 \frac{7}{\text {. }}$ B̈. 2,2. (Entftelst ebento aus Siupfertes.

3 inevitriol. Fryftalle undeutlid, zerfallen an Der suft,

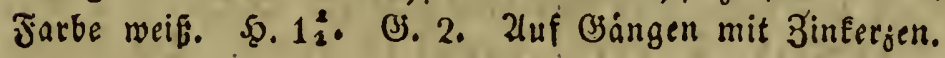

3weite Stonung. Salz=Steine.

§. 180. óden fich-gar nicht (nur ber (5ipps etwas) im Şaffer auf, find burchfichtig bis burchicheinend, feltener un= ourchfichtig, meiftens weís, bisweilen bunt; beutlich fryftalli= firt. Scárte 1-6. (Semid)t 2,5-4,5. BBefteben aus (Froen

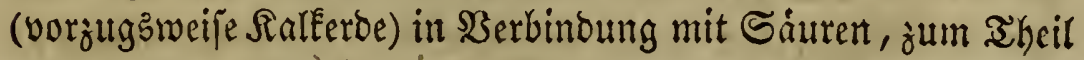
mit beigemengten färbenben Metallornden; manthe finben fich Derb in grofien $\mathfrak{L} a g e r n$ als ganje (3ebirg jormationen.

\section{A. Salferbe haltige.}

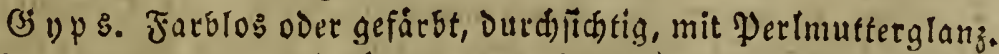

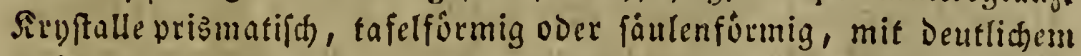

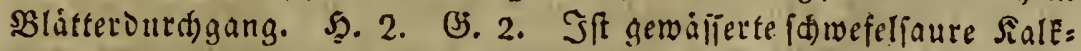
erde. Findet îं als Biebirgsgeftein int jüngeren Jróggebirge und in vielen 2tbánderungen, ftrablig, faferig, Diaft, erdig; Dergleiden find Jafergyps, Jea ueneis und al labafter. 
Salefp ath; Ourdiafeinend bis burtilidrig, weip ober bunt.

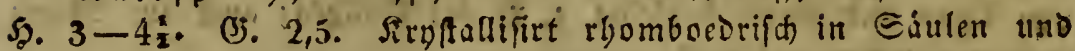
Doppclpyramiden, fpiłen fedbleitigen oder fladen orelfeitigen; befon: Der's Derb it gropen Daffen als Ëesirgsgeftein. Sit Eoblenfaure Siale: erde. Rbánderungen viele. Sireide, Narmor, Eefonftein, SalEftein, bituminofer, Mergelfdiefer, Mergelu. igl:

J゙ Durdafidtig, farblos oder blau, griu, roth. 5.. 4. Bj. 3,3.- Tin= Det fidh auch blittrig, Didt und erdig, befonters auf bsangen. Sft fugfaure Sialerde.

2Cp a tit; Ervplallifiet in gleidffeitigen fed)sfeitigen Sáulen, obet Derb; undurdylidtig. biz Durdfidstig; Jarbe blau, roth, gelb, braun; S. 5,$0 ;$ (3. 3,0-3,3. Brud inufdelig, Fettglang. Sn alten Bies birgen eingefprengt und auf lagern. Sit phosphorfaure fialeerde, Pgoeploreuscirt.

B. Strontian = uno baryterbige.

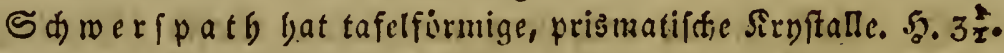
3. 4,5. Farbe weißs, graulid. Findet fich ftrablig, fórnig, ftenger lig abgefondert uts Didt. , Sit finwefelfaure Baryterde. Bolog: nefer $\mathfrak{R} e$ ud titein.

C. $\mathfrak{Z}$ fon $=$, Eale $=$ und Fiefelerbige.

Sind unmidtig und finden feine befondere 2nnenoung; viele

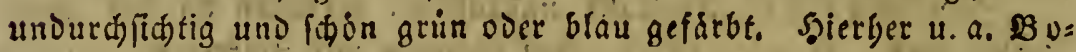
ragit, Datolitg, Túrts, sajulitg u. Dgl. Dle Jarben meiftells metallifden urfprangs.

\section{Dritte Sromung: Salz= Metalle.}

§. 181. Qojen fich nicht im $\mathfrak{B a f f e r}$ auf, find unourch= ficktig ooce an ben Sinten burch fheineno, uno weiß oder bunt=

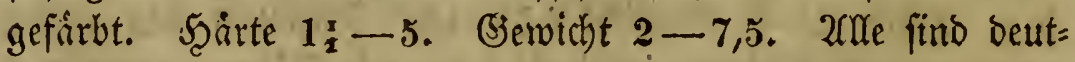
lich Eryftallifirt, boch meift prismatifh. Gie beftehen aus ge= fäterten Metalloxyden uno finben fich auf (3ängen, feltener in Eagern bez álteren Frögrgebirges.

A. B leibaltige. Jatbe weif, getb, grin und roth, $\mathfrak{s}$. $2 x-4$. B. $6,0-7,5$. SBrtd) mufderlig, Bjlas: bis Demantglan, Deutlide Sirnftalle, Pribmen oder Tafern. Jinden fid auf Bángent in Befellfanuft yon Bleierzen, uno befteren aub Bleiorvo mit ver= fobiedenen Sifuren; weí, Eoblenjauer, bellgrün pgosphorjauer, roth dromfauer, getb molyboanfauer u. f. w.

B. $\mathcal{B}$ u p fergalt ige. Farbe Duntelbrau oder grin. $59.3^{\frac{x}{x}}-5$. 
Erfte Slaffe: Salinifate Gefteine. Salz: Steine.

(3. 3,6-4,3. Etrid gefárbt, ater beller; B̈lanz matt; Deutlidjè Siryftalle, over frrablig abgefontert in sinollen und Nieren. 2luf

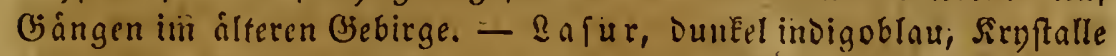

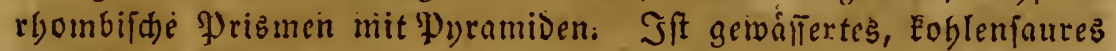
Siupfer: - Mial a dit; fpangrün, faferig; in Siteren; i if ftárfer gewáffertes Evoflenfautes Siupfer.

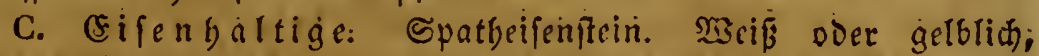
braun meroeno an Der Ruff. Siryftalle Nigomboeder. 5. 4. \$. 3,9. Sfte kohlenfaures Cifenorind.

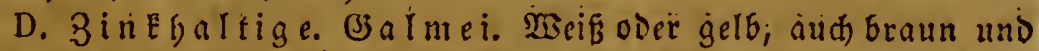
gruiti. Sg. 4,5. (5: $3,6-4,6$. Siruffalle rgombocorifक. Sft Eob: lenfaures 3inkorno; fanil jt yor Dem Sithrohr ju (Email. Jindet

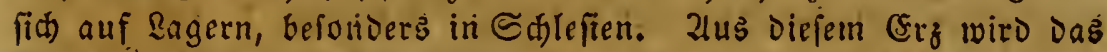
meifte 3 in exetall gemoninen.

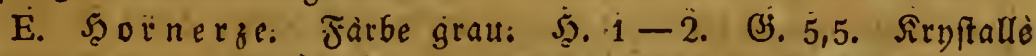
ziemlid Dentlid, oftaedrifa. Şierger Das far faure Sueckffiber; beibe felteit.

\section{3we the flafít.

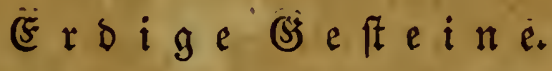

§. 182. Sie baben viele áußere Jennzeichen ber vori: gen, alle Şârtegrabe $(1-10)$; aber vérhâltnißßmás̄ig ein ge=

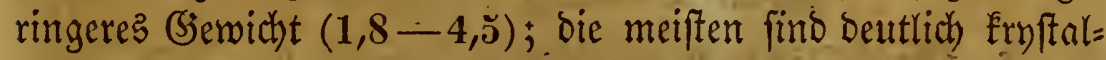
lifirt, warferkell ooer bunt gefórbt in allen (Graben' ber Durct)= fichtigêteit, viele, bejonoers undurchfichtige, erfecheinen Eerb als

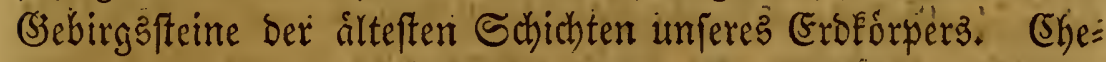
milch) beftehen allè aus Eroen, theils rein; theits mit anderen ober Metalloryden verbunder.

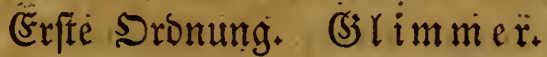

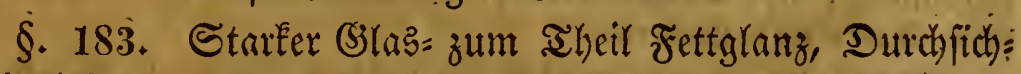
tigkeit bei oft bufferen Farben, untergeoronete Sirnftallifation, aber beutliche Slatterburchginge, eine Scârte von $1-4 \frac{x}{2}$ uno (jervicht von 1,8-4,3 jeichnen bie. (Siruppe aus. "Sn ihnen berrichen $\mathfrak{X} a l^{2}=$ und ahonerbe mit Metalloryben vor.

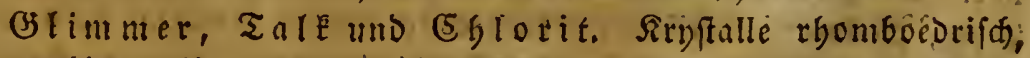

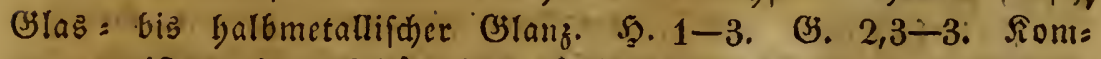
men meiffens in tafelfórnigen firiffallen mit farế entwickeltem

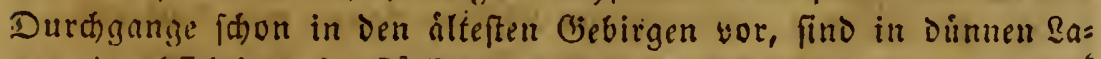

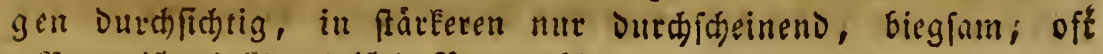
\$Bแm 


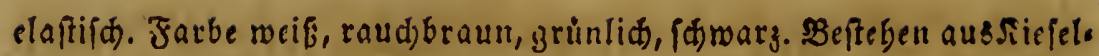
Tbon = und Talferde mit etwas Eifenoryo. Man Eennt vicle Jors men. 3. B. E ry ft allifitter T a IE, woyon ber blattrige Tale und Der Talefdiefir abanocrungen find, gemein bieglam, febr fettig anjufüblen, ourdhideinend; ex entbolt keine Thonerde. Der bs lims in er oder \&epicolitb) Dagegen entbält keine TalkerDe, ift elaftifd bieg. fam uno findet fich mur felten Eryftallifirt in grofen ourdhfidtigen Dlatten, Niariengla 8. (5glorit, gemein bieglam, unourchlid): tig, fettig; Jarbe grin; Perlmutterglanz; weidjer als Bilimmer. 2Ils Bementigtbeil und auf Bingen, feltener eroig oDer fdieferig (Shloritjdirfer). - 2lbinderungen Des (jlimmerz, die jùm Theil als Bebirgżgefteine auftreten, find: Der Tbonfdiefer, Tafelfdiefer,

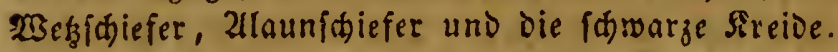

Urgillitbe oder Thone. (E: fino unÉryftallinifde Maffen ogne Spur yon Etruftur; Brud erdig und didt, geringife Şárte: grabe. (3) 0,6-2,8. Unourdithtig, in allen Jarben; theils mager, theils fettig anzufiblen. Defteben ebenfalls aus siefers, Thons und EalEerde. Shierber eine Rienge Foffilien. 3. 15. Der al u min it, oder Die reine Thonerde. Die $\mathfrak{P}$ or zell a nerde, Der Tripel, Der Silebfuicfer. - Der Jeuertbon, Die $\mathfrak{B}$ a dé - Der-gencine Tyon, Die Ge!berde, Der Dollls, Die Bergfeife, BBalfererde, Brin: erde, Deerfda a m, Der Specffein oder Die fpanifde Jreide u. a. m.

\section{Sweite Sronung. Spathe.}

§. 184. Dichte, unourchjict)tige (jefteine von Fett=, (Slnלss ober \$Jerlmutterglang. SBunte bod) meift bunfle Farben. 5.

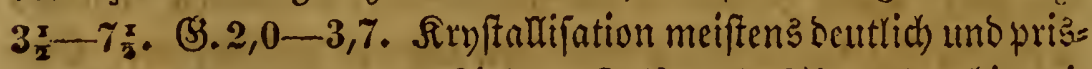

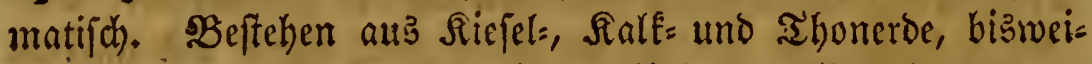

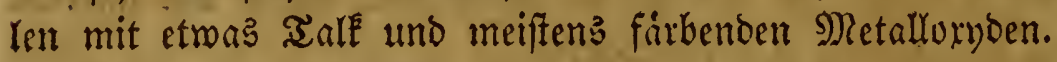

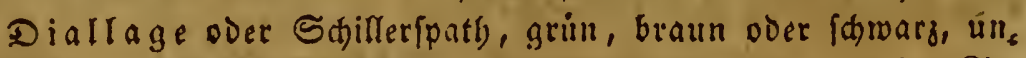

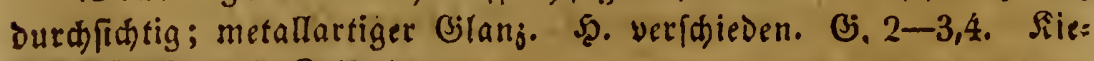
fel=, Tale: und Salferde.

3colitbe, Jarbe mift weif, undurchfidtig. S. $3 \frac{1}{2}-6$. B.

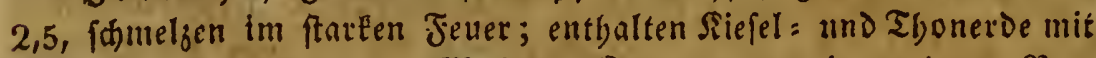

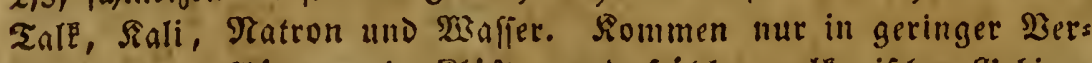

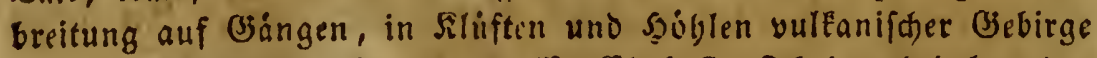
yor. EEs gicbt viele Formen, 3. B. CGabalit, Jathyophthalm ooer albin, Mefotyp, Etilbit, Blätterzeolitb, Reujit, 2nalzim u. a. m.

Felofpatye. Sgelle, zum Zgeil faillernde Jarben, meiptens

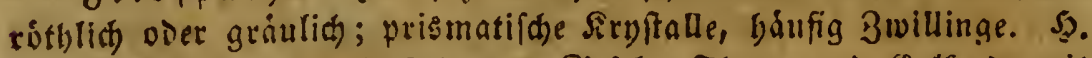
5-6. O. 2,0-2,8. Befteht ans Siefels, Zbone uno Sfalecrde mit allealien, und finder fid in grofer $\mathfrak{z e r b r e i t u n g , ~ b e f o n d e r s ~ i n ~ D e n ~}$ 
alteren ভdidten, 3. B. Im Granit, Bneus, yorpgoren u. v. a. Es giebt viele Jormen, Die jur Giruppe des Feldipatgs gebiren, $2 . \mathfrak{b}$. 2loular, Epapolitb, Pefalitb, Epooumen, Rephelin, Drebnit u. a.m.

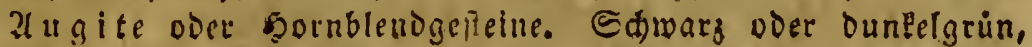

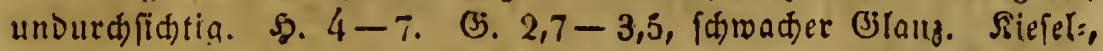
Salk:, Tyc: = uno Talêtoe. 2118 Bemengtbeil dlterer Gebirge,

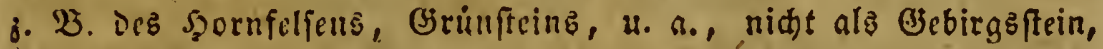
immer elngefprengt in Eleineren Partien. X'banderungen zablreid,

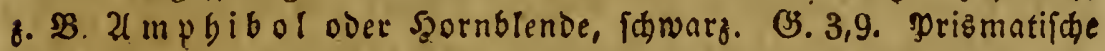
Sryftalle. - 2uzit, grin ober fhmarz, Fivffalle yierfeitige Dris= men mir Pyrauise. (5. 3,5. - P ift azit oder Epioot, bellgruin, an Den Santell sum Theil Durchideineno, geringere \$2.; beide fitmels bell vorm Bitgrebr. - 2l s beft, foferig, biegiall, weid, meifgrun bis ins Grune. - Serpenti $n$, Derb, faft erdig, febr meid, grủn.

Dritte Sronumg. Edelfteine. Bsemmen.

§. 185. Selle, burchfiktige, Leutlich fryftallirte (S)

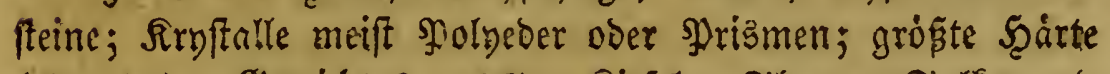

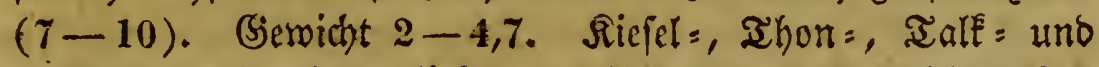
Rallerde, mit vielen färbenden Metalloxyden; meiftens fernz ftallifirt. 2fuf - (Jiangen in álteren Giebirgen, oder als Giee fchicbe, bismeilen berb als grope (siebirgşgefteine, boch fommen fo nur Soltarz uno Iupas nor.

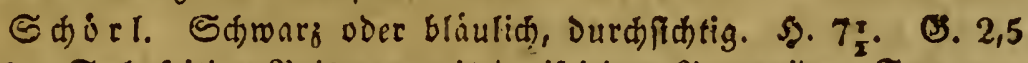
$-3,5$. Eedsfeitige Wrismen mit oreifeitigrr Pyramide. Turma=

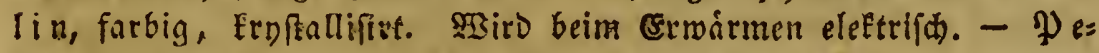
lip un, blau mit Didroiftifutem Jarbenmedfel, leidfter als voriger, felten Eryftallifirt, meift als Befdiebe. - Égryfolith, Drivin,

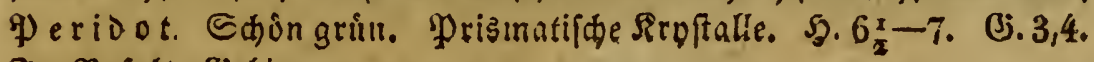
In Bafalt : Bebirgen.

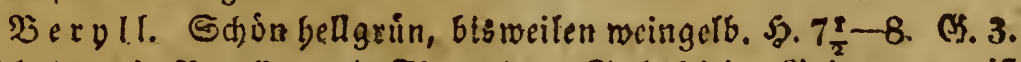

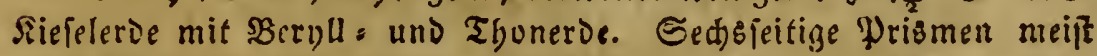
ogne Pyramiden - Jlidien. - E U Elas, DunEler grún, Poramiden

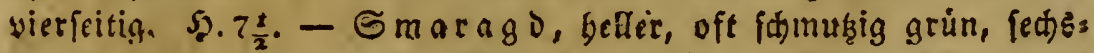
feitige Pyramiden. S2. 8. Befonders fain in Sildamerika.

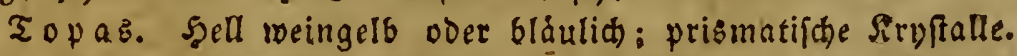
57. 8. 35. 3,5. ?trs Bebirgsgeftein ain Ednedenfels in Sadjen, fonft auf Bängen in Siliften, befonders fajón in Barafilien.

Q $u a \mathfrak{r}$. Wafferbell und in allen Jarben, ledbefeitige \$rismen

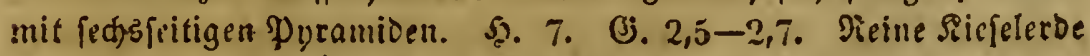

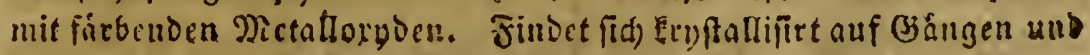

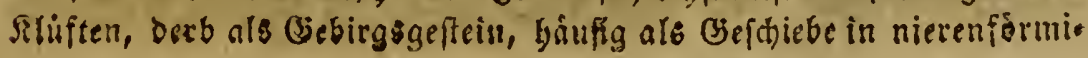


gen Stücen. 2(bänderungen find: a) freftallifirte: wafferbell Berg:

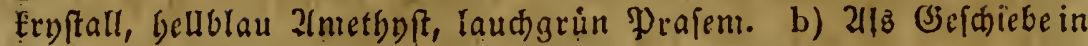
Sinollen: grau Feuerftein, roth Earneol, grin (5)rofopras, in med)= felnoen, grau, braun und weisen ङtreifen Suyx; balb Durdfithtig,

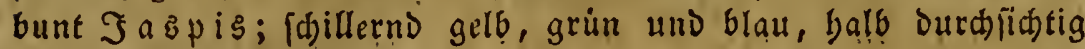
Spal u. Dgl. $\mathrm{m}$.

(ङ) ranate. (j)

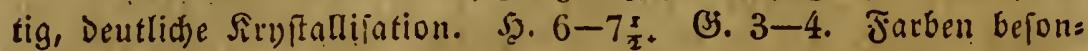
Ders Eaneel= oded blutroth. Fiefel:, Thon s und Sạkerde. Findet fid) eingefprengt in alteten Bjebirgen, aud) in vulennifhen, befonders Bafalt, uno als Geidjitbe. 3. B. Sta u r ol tit b, braun, ungleids= frosfcitige Drismen, oft Ereuzmeiz. - Defuy i an ober Sookras, fdmutsig grun oder braun, pyramidale firyftalle. - Sgelvi u, fdmus

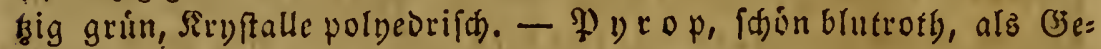

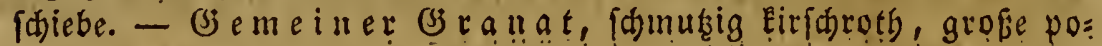
lyeorifue Sirvftalle.

3irfon, rotb ober scaun, Durdfidtig, firyfalle vierfeitige

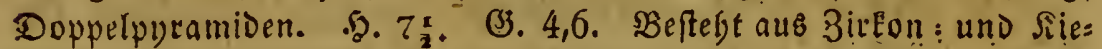

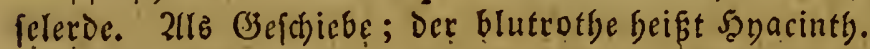

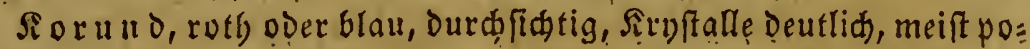

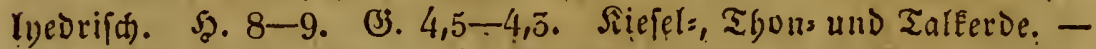

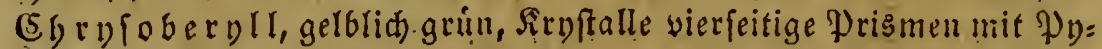

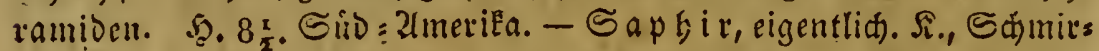

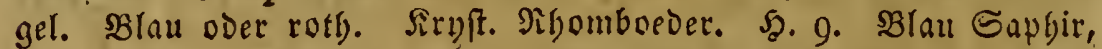
rotb Rubin. - Spinell, weinroth, (d)warz odẹ fdmuţig grú. Sirvit. DEtacder. SS 9. Sevlout.

Dem a nt oder Diamant, rob graubraun bis mafjerbell, ge: fdliffen mafierbell mit lebhaftem Jarbenfpiel und Demantglanz.

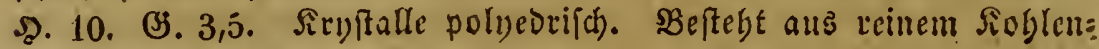

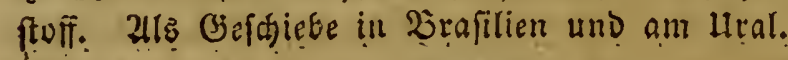

\section{$D \mathfrak{i} t \mathfrak{t}$ e $\mathfrak{i} \mathfrak{l} \mathfrak{a}\{\mathfrak{f}$ e.

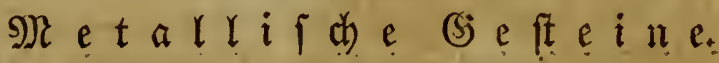

§. 186. Der eigentbumliche (SIanz, die lebbafte Får= bung werbunben mit lnourchfichtigéteit, geringerer Scárte, aber bedeutender Schwere, unterfcheiden diefe (siruppe. athe find im Feuer famelzbar, und lafien fich) baburch, felbit menn fie mit anderen Stoffen verbunben find, rein (gebiegen) bar= ftellen; doch fommen mur wenige, meiftenక̦ bie eolen s)letalle, gcoiegen vor. Wule finden fid) auf (siangen uno lagern in alteren (s)birgen, ober auch eingeiprengt in jungere (5rofichich); 


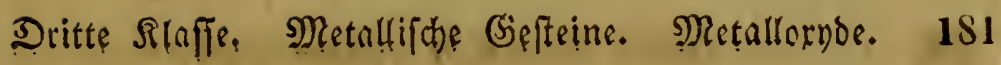

ten. Man fennt gegenwairtig 29 verichiconene Metalle, die prybint, gefchmẹelt ober rẹin vorfommen.

\section{Erfte Oronumg. Detarl $=$ S ryde.}

§. 187. Sie haben meiftens cinen fchmachen Metall= glanlo, bunt gefórbten Strich), bie grófte Scarte (bis 7) uno bas geringfte (jetwicht $(3,4-7,4)$. 2ale befteben aus Metall mit Sauterftoff verbunben.

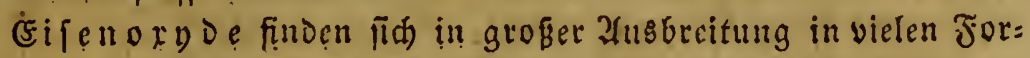
mationen Der Eroe; fie baben eilie braune oder fdwarge Jarbe, ei=-

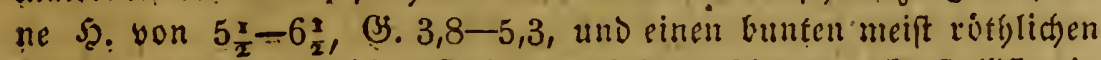
Etrid: Nagneteifenfein, tief braunfdwars, kryfrallifit in

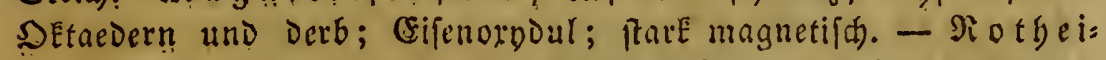

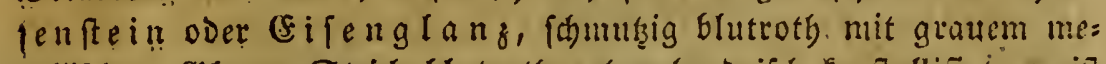

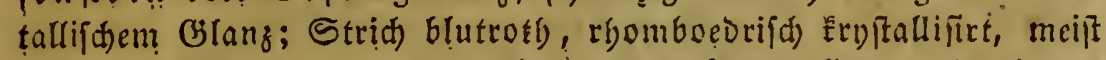
ftraglid abgefondert in nierenfórmigen Stücken; Cifenorno mit an=

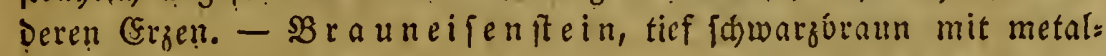

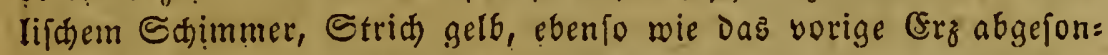
Dert; Eifenornd mit Waffier. - Eifen findet fid) gediegen nur in Den Meteorfteinen.

IR anganoxybe, fdrarz oser braun, Strid ebent fo, zum Tbeil glanglos, faft erdig; $5.4-4,8.52 .2 \frac{1}{2}-5 \frac{x}{2}$. -5 d) warzer

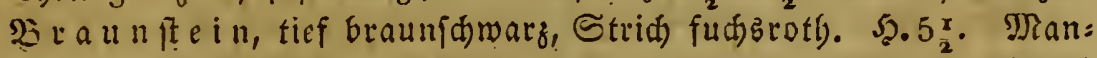

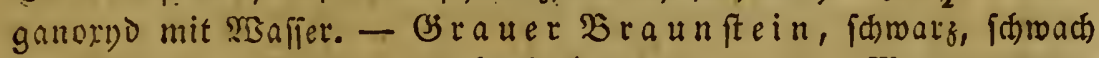

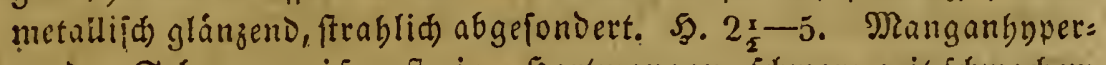
ordo. Sdwarzeifenftein, Sartmangan, fitwarz mit fdwadfem Metalglanz, ebenjo abgefondert mie die S3origen. CEifenoryo mit Manganoryd. Das granganmetall, weldes Dent Eifen ábuclt, Eommt, wegen feiner grofen? Serwandtf gen yor.

3innftein, famarg, Strid graulid, Siryftalle quatratiféce

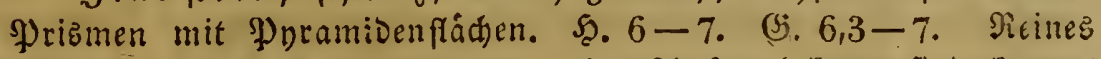
3innornd. Dos Binmetall, Deffen (Eigenfdhaften bekannt fint, Eommt in Der Siatur am meiffen in Diefem orndirtên 3 uftande yor.

\section{3weite Sronung. Metallénige.}

§. 188.. Sie haben Deuttichen Metrilglanz uno lichte metallifhe Farben. Sirnftalle nid)t fehr Deutlich, meiftents

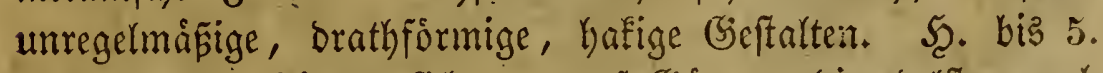
(5). 5,7-21. Finden fich nur auf (3ingen, bie coelften aud

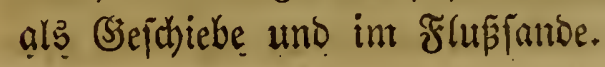


Spiefgla ns, 2lutimon; undeutide rfomboedide sirypalle,

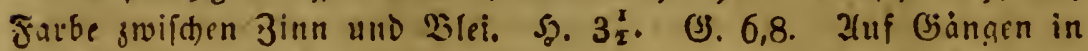
Jranercid), aber felfen; ift fludtig im Jente; miro zur Compointion Des Retternuctalls verivenict; fintet lich auch gefthefelt.

T2 is an utb, undeutliche, tetraedrifhe Siryptalle, Jarbe zinnmeiff,

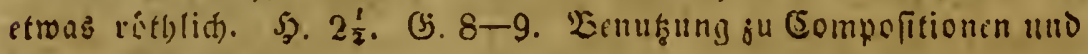
in Der Jiedicln.

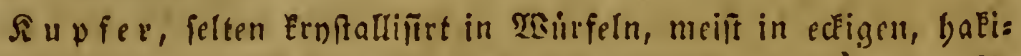
gen, plattenartigen Maffen; Farbe bell braunrotb; $5.2 \frac{1}{2}-3$, (3.

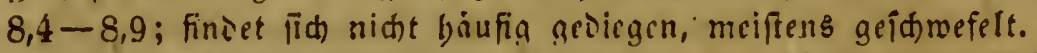

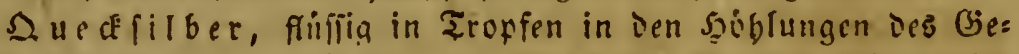
fitins, gefriert bei $-31^{\circ}$ Reaum., flibermeif; (\$) 15,0 ; flidtig-im

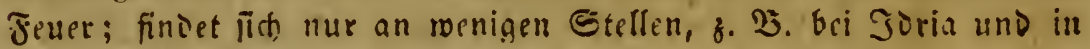
Deru; wiro ;ur Epiegelberfitung uno zum 2ergoloen binukzt.

Gilber fintet lith expitallifirt in Detaebern over in unregel= mafig geformten, gepriciten, baar = oopr nabelfirmigen, aud bakis gen giafien auf (siangen in älteren biebirgen; Farbe fdmukgig grall, gereinigt weisis; 5. $2_{2}^{2}-3$, (5) 10,0 bis 10,5 .

(jo to findet fids nur gesiegen in firnern, Dritgen oder Eleie nen Piatten, feltener in oftacorifden firyftulfen, auf Bingen, over

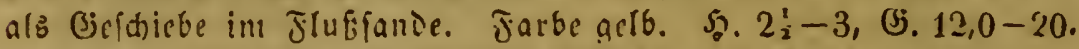

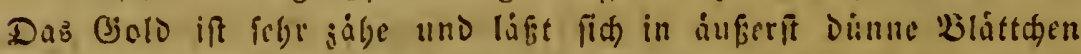
fhlagen, Dann fálmmert Das sidht grin bindurd).

P l a i in, unregelmifig geformt, in Siornern von grauer Jarbe,

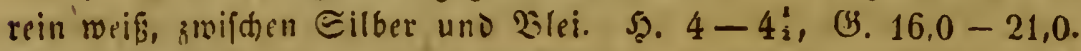
çbcufall bánimerbar und fdmeip̧ar, mie bas (sifen. Choamerifa und ain liral.

Dritte Sromung. Schrefelmetalle.

§. 180. 2fuch in Diefer (siruppe iff ber Mctallglanz uno

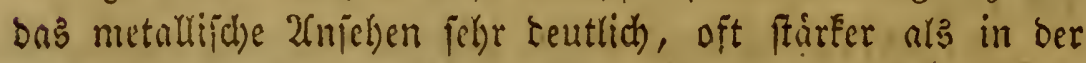

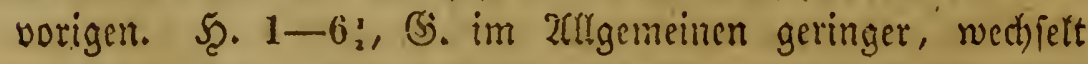
won $3,5-3,2$. Ifle beftên aus Mnetall mit Echwefel in verfa)icuenen (siraben gemengt. Die Meiften fino bentlich Ernftallifirt, s]lanctye frablich abgejonbert in Sugetn uno snolren. Sic finbeir fich auf (s)ingen in alteren uno cin= gepprengt in jingeren Formationen. In ber s) Retallurgie

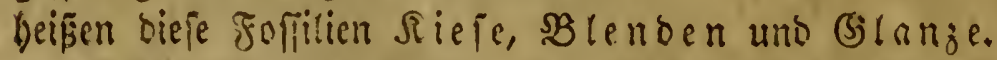

S di re felcifen, Edwefeleies. Mrefingaglb, an ier Buft braun anlaufend, frifó mit frarkem 9ietallglanz. Sirgifalle polycorifa), ober

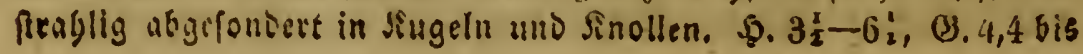




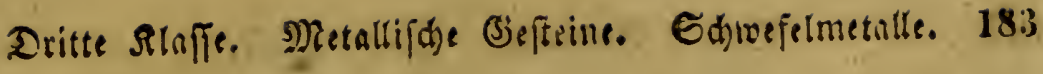

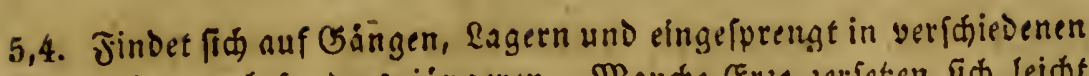
Formationen, befonders jüngeren. Mandje (Erze zerfeten fid leidts an Der Ruft, und bilien Dann Eifinvitriol. Sit Eifen mit Edmefel.

S d) wefele upfer. Sirypallifation untergeoronct, meiftens in Derben Etidfen. Farbe wedfelnd jwifhen idmarg, braun, gelb, rotb;

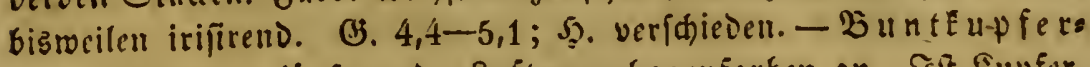
er b, rotbbiaun, lauft an Der Ruft regenbogenfarben an. Sit Supfer,

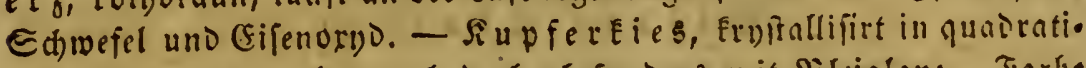
fhen Tetraedern, aber aud Derb, befonders mit Dreiglang. Jarbe fain gelb, beller als Eifenties. Sft Edmefileupier mit Edircifs

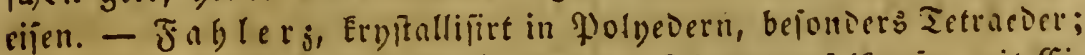
ftablgrau gefäbt mit unebenem Brud). Sit SdmefelEupler mit (⿶): fen, ?r reniê uno Epię̧glanj.

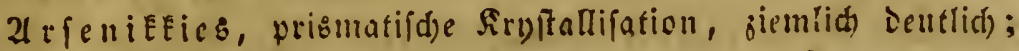
Jarbe frifh filbermeif, an ier Luft grau merient. S.5-6, (j). 5,7-7,4. Deptegt aus Edwifeleifen und ItreniE. - Das 2trienif= metall bat eine weifgraue Jatbe, eia 35 . yon 5,8 , eille 5 . bonl 3,5 , iff fluditig, uno riedt Dabei wie Sinoblaud; ; es findet fich in ier Siatur audh geviegen, aber meiftentweier an Sauerftof wier an Edweifil ge: bunden. Wit erferem bilset es die fibr giftige weife arfenige Eiure; man gebrautht $t$ 's ou (E)แpolttionen uno in ier Micticin.

fabalteies (Epeiftubalt), Siryftalle oftnesrifh, and ftalal's tifde Formen, Jarbe jinmweif, grau angelaufen, Bruch uncten. Sg. 5,5. (5. 6,5. Zuf Singen, bejtebt aus Siobalt, ?trienie uni) etwas Edwefeleifen. - Das fiobultmetall findet jid meif mit 2lrfenie ver: bunden, bat eine bleigraus ins gitithlide gebenie Jarbe, grobtirnigun

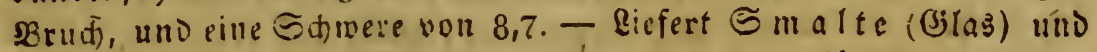
3affer (Drvo), erftere bla u, lesegterer gelb gefárbt.

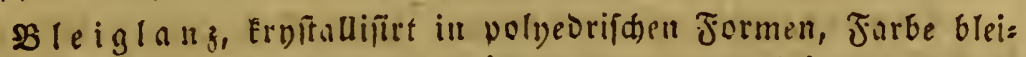

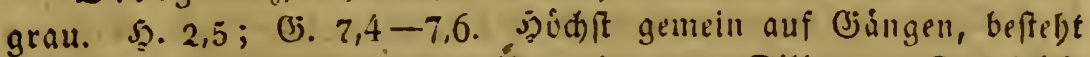
aus Edrefel uno slei, bismeilen mit etwas Eilber. - Das Blei= metall finoet fich in Det Oatur Eaum rein, fondern yorjugeroife in Diefem gefdrwefelten 3uftande.

Spief́glang, prismatifde firufralle, meiff frublig abgcion: Dert, Farbe DunEel bleigrau. So. $1 \frac{1}{2}-2 ;$; $4,2-5,8$. Sit Efiet:

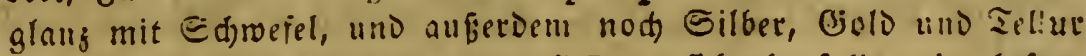
enthaltend. Das Epiefiglanjmetull finiet fid ebenfalls rein, befon: Ders aber geidhefelt und oridoirt; es ift jaber als Blei, abcr lidids ter; man braudet es ju Compofitioncil.

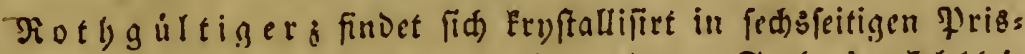
men mit Pyramiden, oder ftroblid) abgefontert. Farbe Bunfel blei: grau, gegen das fidet gebalten blutroth Durdideinend, Strid rotb. 


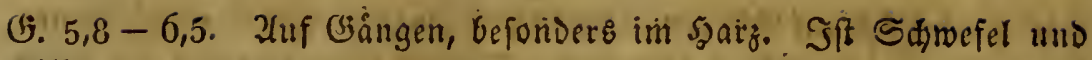
silber.

3 innober. Sivnftalle undeuflid, meift ftrablig abgefondert;

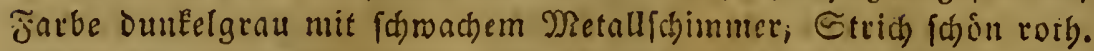

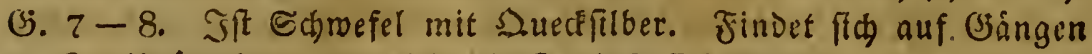
im 3meibrickidgen und roird als Farbeftoff Benuşt:

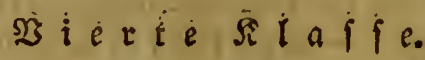

Brenzliche Gefteine. Brènze.

§. 190. Fett= ober Silasglanj, unseutliche Sryftallifation;

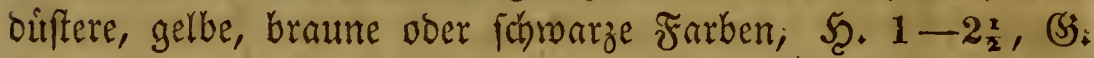
1,0-3,6, und bie leidste Serbrennlich Eeit im Feuer mit ei= genthimlichem (Siputh charalterifiren biefe (s)uppe. Einigè find elementar, wie ber Schmefel; andere befteben auts Soh)

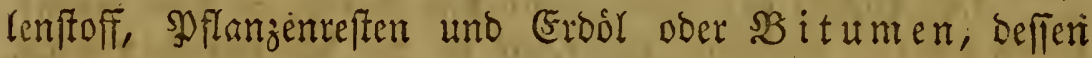
eigentlumlichen (Sieruch manche beftiser. Sie finden fich nuf

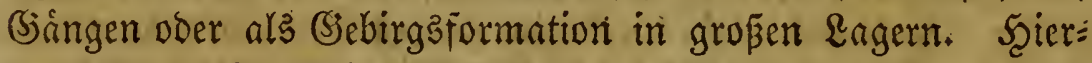
ber nur wenige Mineralier.

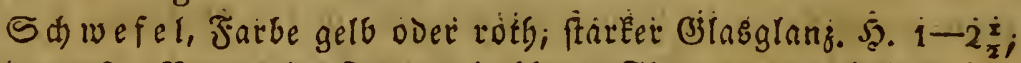
(3. $1,9-3,6$. SBennt im Jeuer mit blauer Jlamme uno riecht Dabet:

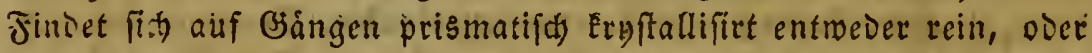
mit 2 trfenil verbunder als Sperment in Der zreiten, und als Realgar ober Sandara in Der erften Sdynefelmifhung.

(ErDil, (ErDped); 2liphalt, bat eine braume Farbe, ift frif fig, leidfer als Wafjer und riç)t ftarE; quillt aut Der Croe, befon= Der's in Palaftini am todten Jieer:

foblen, fómarí ober brauri, Glasglanz oder erdig, nicht

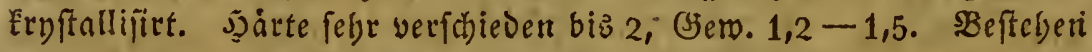

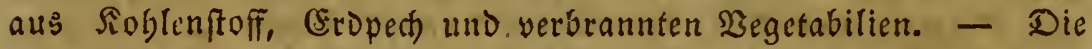
StcinEoble ift fdrarg; glangent; nie erdig, feft, und fincet fid

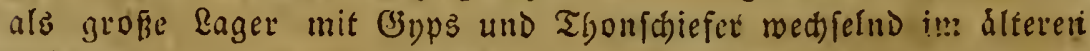
Florgebirge. - Die $\mathfrak{B r}$ a un E o g le ift braun, glanjloz, erdig, bat oft nod) Solbifruktur; und findef fid mit Sand uno Mergel nbwed)=

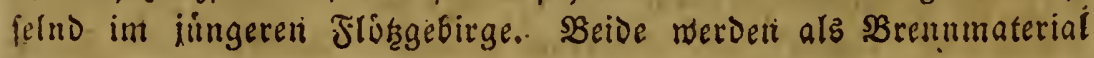
be แuşt. 


\section{$\mathfrak{A} \mathfrak{n} \mathfrak{h} \mathfrak{a} \mathfrak{n} \mathfrak{g}$}

\section{Ueberfidt ber erwåhnten Gewådfe nach Dem naturlichen Syftem.}

\section{Dicotyledoneae.}

(3) beffegt, Deren BBăatter artifulirt fint uno neśfórmige Mippen haben. Shbre Bghumen zeigen einen Feldh uno meifens auch eine Srone; bie Samen einen beutlititen Seim, mit zwei ober mehr Samenlappen. San ben Sorganen berrfdtt bie Rahl fünf ober vier.

\section{Erffe Ritalfe. Polypetalae.}

Die şlumenfrone ift ftets borhanden und befteht nus mebreren $\mathfrak{B l u m e n b l a ̊ t t e r n . ~}$

1. Dronumg. Thalamopetalae. Die Sironenblätter

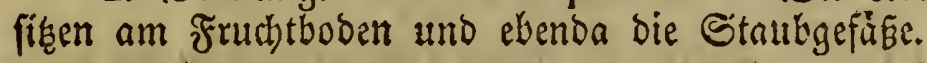

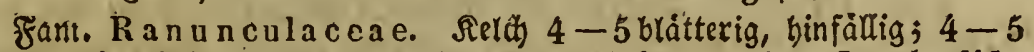

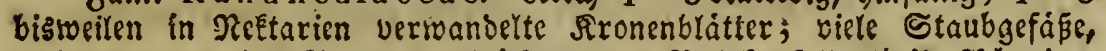

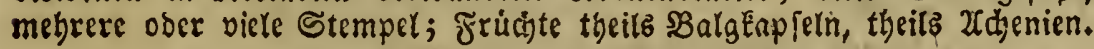

Batt. Delphinium 138, Aqquilegia 139, Helleborus, Caltha 139,

Ranunculus 139, Adonis, Clematis, Thalictrum, Anemone.

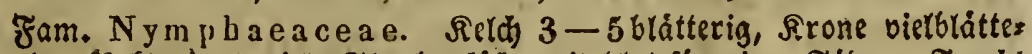

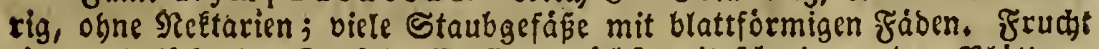

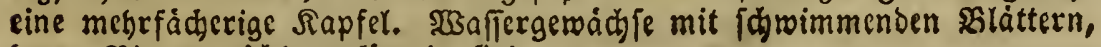
beren Rippen nicht neegformig find.

Batt. Nympliaea 138, Nenuphar.

Fam. P a p a ve r a c e a e. Ficth 2 brätterig, Ginfállig; Sirone 4 bláte

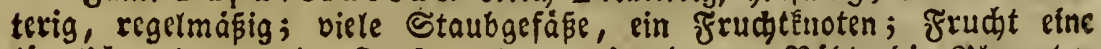

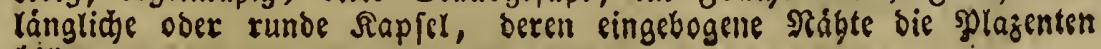
bilben.

Surmeiffer’s Grundrif D, Naturgesch. 
(3)att. Papaver 138, Chelidonium, Glaucium.

Fam. F u maria ceae. Feld 2 blảtterig, binfällig; frene 4 blảts terig, unregelmákig; frud)t ber Borigen, oft 1 = bis 2 famig; 6 monas belpbifde Śtaubgefábe.

(Satt. Fumaria 143, Corydalis.

Zam. Cruciferae (Tetradynamia). S. Seite 141.

Zam. Sterculiaceae. Zutrermeift Báume; Reld und Frone 5 blåtterig, bie $\mathfrak{B l a ́ t t e r ~ a m ~ S r r u n b e ~ v e r w a d j e n , ~ e b e n f o ~ m i t ~ b e n ~ S t a u b s ~}$ gefáß̨en; biefe monabctphif h), einige obne, bie anberen mit 2 fádjeriger Znntbera; Frudht eine mebrfáderige, vielfamige Siapfel.

Shatt. Theobroma 145.

Fam. Malvaceac. Fráuter ober Stauben; Felch Doppelt, ber innere roie bie frone 5 blátterig, bie slátter unter fich uno mit ben Etaubgefápen virwadjen; biefe fệr zablrcid), mit 1 fácheriger 2(nthera;

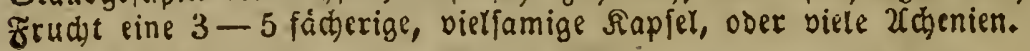

(3att. Maiva, Lavatera, Althaea 143, Gossypium 143, Sida.

Fam. A u rantiacea e. Báume mit Yeberartigen, urúfígen Bláts

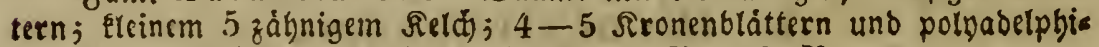

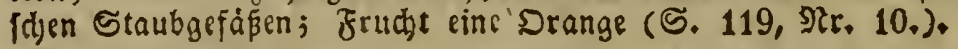

Batt. Citrus 146, Limonia.

fram. H y pericene. Stauben ober Stráucher mit gegeniberftec

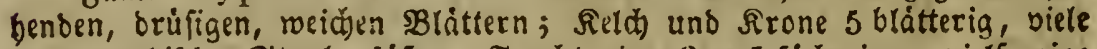
polnabelphifde Étaubgefåpe; Frudbt eine $3-5$ fådberige, vielfamige sapfel.

Batt. Hypericum 146.

Fam. Hippocastaneae. Såume mit gegenúberftefenben, ges

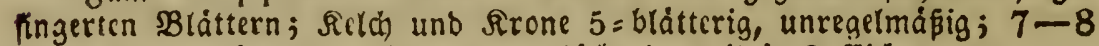

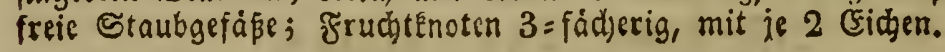

(3att. Aesculus 132.

Fam. A cerineae. Bảume mit gegenuberftelsenben gelappten

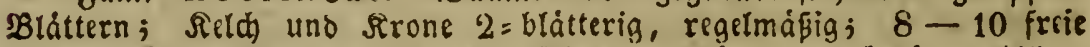
Etrubgefäpe; Frud)t aus 2 verwadjfenen, geflügelten 2 dhenien gebildet.

(5att. Acer 156.

Fam. Li nea e. Firáuter mit einfachen sBláttern; Field̆ und Frone

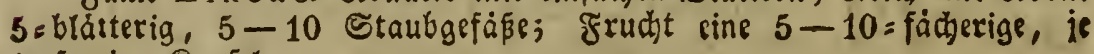
$1=$ famige Rapiel.

(3att. Linum 130, Radiola.

Fam. Geranieae. Friuter ober Stauben mit gelappten Brads

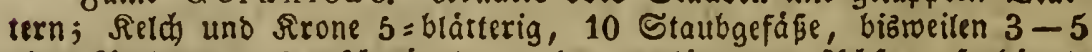

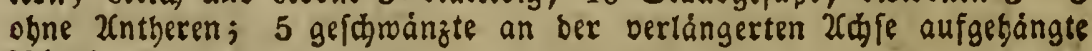
X®enien.

(Batt. Frodium 142, Geranium 142, Pelargonium.

Fam. Resedeae. Irảuter ober Stauben mit fieberfpaltigen Bláts

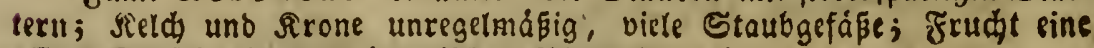
offene Sapfer mit 3 peripberifonen slazenten.

Batt. Reseda 136.

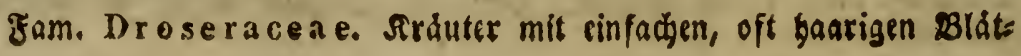


tern; Reld und Rrone regetmåpig, 5 =blätterig; 5-10 Staubgefảpe; Frudt eine Rapfel mit $3-4$ periplerifchen झु!agenten.

(S) 5 att. Parnassia 130, Drosera, Dionaea.

Fam. Jonideae. Fráuter; Reld und firone $5=$ blatterig, unres gelmáfig; 5 Staubgefáfé; Frudt eine 3 = flappige Sapfel mit şolağens ten an ben Slappen ober im (S) runbe.

(satt. Viola (Beildyen).

Fam. Caryo phyllea e. Siråuter ober Stalloen mit gegenúbers

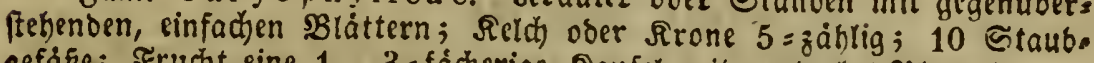
Befảpe; Frucht eine $1-3=$ fáchcrige fapfel mit zentraler Plazenta.

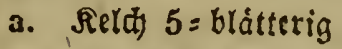

(3)att. Stellaria 134, Arenaria, Spergula, Cerastium.

b. Risth rỏbrig, $5=$ zábnig.

Batt. Silene, Lychnis 135; Saponaria, Dianthus 134.

2. Dronung. Calycopetalae. Die fronenblätter uno

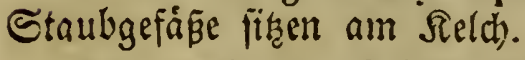

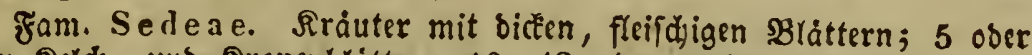

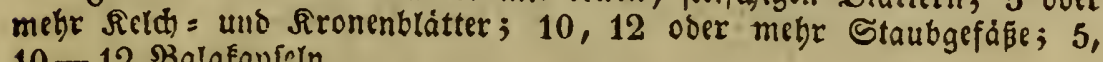
10- 12 Balg Éapietn.

(Satt. Sedum 135, Sempervivum.

Berwanbte gamilien find bie Cacteae, Grossularieae, Philadelpheae, saxifrageae.

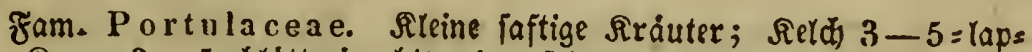
pig; Arone 3-5 =blátterig, bisweilen feglenb; $3,5,10$ ङtaubgefäßs; Fruchet sine mebrámige Rap fel.

Montia 126, Portuiaca.

Fam. Rosaceae. Wflanzen alle: Formen; Seld verfhieben,

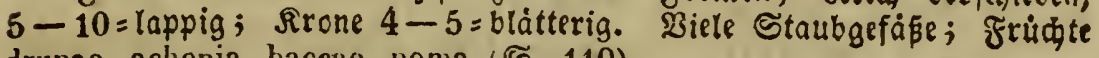
drupae, achenia, baccae, ponia (S. 119).

A. Biełe cingetne frúd)te alts ciner Blume.

a. Frudte ein famig; Ucthenien ober $\mathfrak{B e c t e n}$.

$\alpha$. Field boppelt, $8-10=$ lappig.

(Satt. Tormentilla, Potentilla, Geum, Fragaria 137.

$\beta$. Fieldy einfach, 5 =lappig.

Batt. Rubus 137, Rosa 137, Agrimonia 135.

b. Frúchte me hr r famig, Balgéapfetn.

Batt. Spiraea.

B. Eine einz̧ige, 1-mełráa derige Frudjt.

Batt. Pyrus 137, Prunus 136, Anggdalus 137.

Fam. M y r t a ce a e. Baumartige \$flanzen mit sinfactien oft rebers

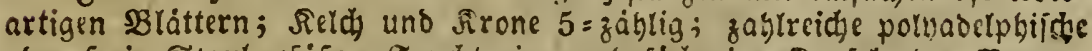

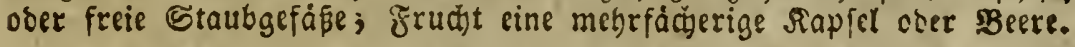

Batt. Myrtus, Caryophis llus, Punica, Melalenca, Metrosideros.

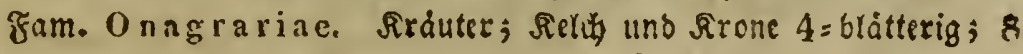




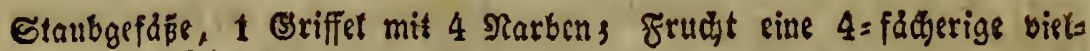
famige Rapfel.

(satt. Oenothera 132, Epilobium.

Fam. Salicariae. Âráuter mit gegenuberftehenoen Bgláttern; Fetch uno Arone 4-6=blätterig, 4-10 Staubgefápe; Frtucht eine meift 2 = fädjerige $\mathfrak{S a p f e l ~ m i t ~ z ̇ n t r a l e r ~ s ̧ l a z ́ c n t a . ~}$

Batt. Lythrum 135, Peplis.

Fam. Le gum in os a e. Srite 144.

Bermanste Familien find bie Terebinthaceae, Rhamneae, Hederaceae, Lorantheae.

Fam. Umbelliferae. Seite 129.

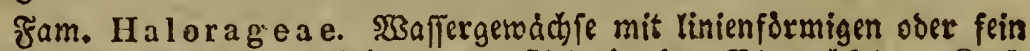

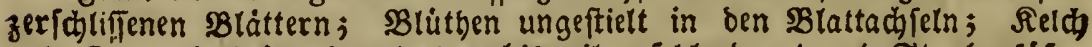
und Srone $4=$ blätterig, leğtere biorveilen feblend; 1-4 Staubgefáfe; Frudt ein 2(d)enium.

Batt. Hippuris 123, Ceratophyllum, Trapa.

Sweite Silaffe. Monopetalae.

Die róbrige, poer trichter = uno glockenfórnige Blumen= frone befteht nur aus einem Shlatt, uno ift am Rande in Eappen getheilt.

3. Sromung. Calyeanthap. Die SBlumenkrone jitge am Relch, Diefer iff mehe weniger mit Dem Fruchtenoten ver: wachjer.

Şierhner bis nidit errábnten familien Cucurbitaceae, Cam panulaceae, Lobeliacea u. $a$.

Fam. C o mposita e. Blumen bidit georängt auf gemeinfamem

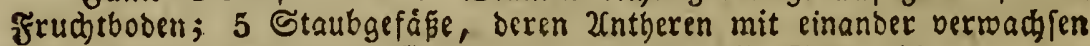
fino; Zrucht tine Siaryopfis. - Syngenesia. S. Seite 146.

Fam. Dipsaceae. Brumen bidjt georảngt auf gemeinjamem

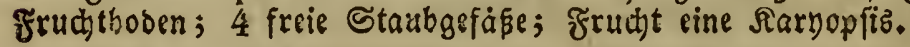

(Satt. Dipsacus 126, Scabiosa, Asterocephalus.

Berwande Familien find bie Globularinea und Valerianeae.

Fam. Caprifoliea e. Stáuter ober Strảuder mit gegenưbcr=

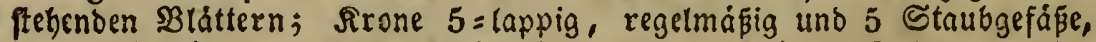
ober rachenförmig uno 4 Staubgefäße; ; Frud)t nidht aufferingend, eine Beere oder Tapjel mit wenigen Samen.

(Satt. Linnaea, Sambucus 130, Vibsrnum, Caprifolium, Lonicera.

Fam. Cinch o neae. Báume mit gegenưberfirehenbent Blăttern

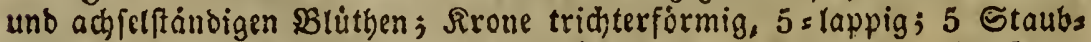

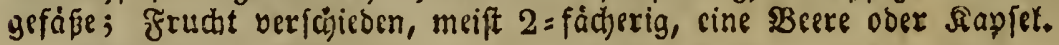

Batt, Coffea 128, Cinchona, Cepplaëlis.

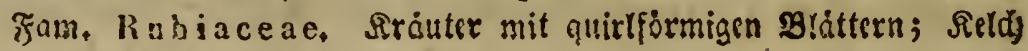


und Frone $4=$ lappig, 4 Staubgefábe; Frucht aus 2 verwahfenen ฝิa= ryopien ober Beeren gebilbet.

att. Rubia 126, Asperula, Galium, Scherardia.

4. Sronung. Thalamanthae. Dis Blumentrone fitst

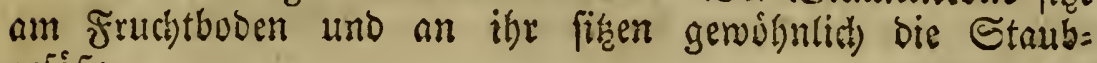
gefáfie.

Fam. Ericeae. Stråucher; Relh uno Rrone 4-5=lappig, 8-10 Etaubgefả̉é; Zrucht $3-5=$ fádjerig, vielfamig, cine Rapfel obcr 28 cere.

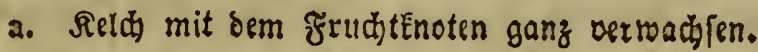

(Satt. Vaccinium 133.

b. Sith frei.

Gatt. Calluna 133, Frica, Ledum 134, Psrola.

Fam. Ole a ca e. SBáume ober Stráuther mit gegenúberftehenden

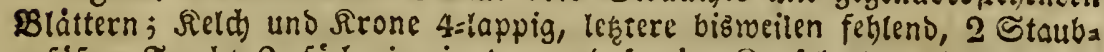

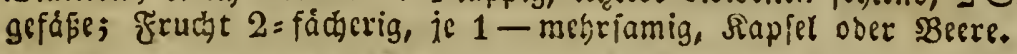

(Gatt. Ligustrum 123, Syringa 123, Fraxinus 157, Olea, Jasminum u. a.

Spier ftehen audh bie nidht erwágnten Farnitien: Ap ocyneae (s. Contortae) und A sclepiadeae.

ram. G e ntia neae. Fráuter mit gegenúberftehcnoen sgláttern; Reld und Frone 5 =lappig; 5 Staubgefápe; Frudt eine $2=$ Elappige, vielfamige Rapiel.

(S)att. Gentiana, Erythraea, Menyanthes 128.

Fam. L a biata e. S. 140.

Fam. Asperifoliae. S. 127.

Fam. Convolvulaceae. S. 128.

Fam. Solaneae. ธ. 128.

Fam. Personatae. S. 140.

Dabin auch sie Batt. Veronica S. 124.

fam. Primulaceae. S. 127.

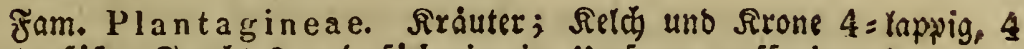
Staubgefäße; ₹rucht $2-4=$ fảderig, im umfange aufipringend.

(Satt. Plantago 126, Littorella.

\section{Dritte flaffe. Apetalae.}

Die BlumenErone fehlt, ber Reldh umbulltt allein bie Staubgefáke und Den Stempel, uno ift balo fronenartig ges fårbt, bald grún.

5. Sromung. Monoclineae. Strubgefäpe uno Stems pel in Derfelben SBlume.

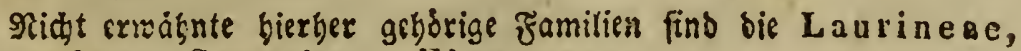
Thymeleae, Santaleae, Eleagneae. 
Fam. A sarineae. Siráuter mit abroed felnben Sláttern; fetch rỏbrig, gefårbt, lappig; 6-10 Staubgefápe; Frud̆t 3-6=fả berig, je vielfamig.

(Satt. Aristolochia 150, Asarum.

Fam. Polyg o neac:. Sirater mit Blatticheiben am Branbe ber Blåtter; Reldł eronenartig, 4-5:lappig; 6-10 Gtaubgefápe; Frucht ein XChenium.

Batt. Polygonum 133, Rheum, Runex.

Fam. C b e n o podl ie a e. Fráuter; Feld ungefärbt, 1-5 Staubs gefäße; Zrucht ein vom ftehengeblicbenen Rielche umbülltes 2 chenium.

Batt. Chenopodium 157, Atriplex 157,'Spiracia, Beta, Salsola, Salicornia.

23erwandte Familien find die Scleran theae, Paronychieac, Amarantaceae.

6. STonung. Diclineae. Staubgefáfe uno Stempel in verichiedenen $B$ luthen.

Fam. Enphorbiaceae. Seld megrlappig, Staubgefápe in uns beftimmter $3 a b l ; 2-3$ verwadjene Stempel; frúchte $2-3=$ fádberig, ie 1-2 = famig, elaftifch auffpringend.

(5att. Euphorbia 136, Merrurialis, Ricinus, Phyllanthus, Buxus. Fam. Urtice a e. ऽ. 149.

Dafin nod Cannabis uno Humulus 152, fo wie bie nidyt erwähns ten (Sattungen Parietaria, Ficus, Antiaris, Artocarpus u. a.

ram. Salicineae. S. 154 .

Fam. A mentaceae. S. 151.

Fam. Coniferae. S. 152.

Dabin nod bie biozifichen (satt. Taxus, Juniperus 154.

\section{Monocotyledoneae.}

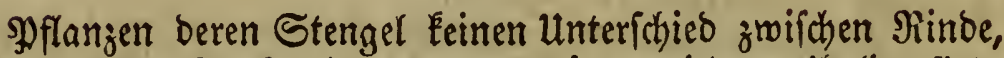
Scolgring und Mtark zeigt, Deren Blätter nicht artifulirt fino, gewodgnlidy einfache ftrablige ober parallele ftreifige Rippen haben; Den Bhlumen feblt die Srone und ber Same hat nur einen Samenlappen. In ihren Srganen herrfdht bả 3ak)= lengefer orei.

\section{Sierte Rlafie. Perigoniatae.}

Die 6 Seldbblatter ftehen in 2 Sreifen, uns find theils alle, theits nur bie bes inneren Freifes Eronenartìg gefárbt.

7. Sronung. Corovariac. 2ule 6 Sherigonblátter finto gefárbt. 
A. Gynandrae. Bon ben 3-6 Staubgefápen fino

1-2 vertummert; frudtenoten unterftánoig.

Spicrber bie Familien Mus aceae, S citumineae.

Fam. Orchideae $\mathfrak{S} 149$.

3. Eipigneae. 3)ie $3-6$ Staubgefápé fino alle fructbar; fruchtenoten untcritándig.

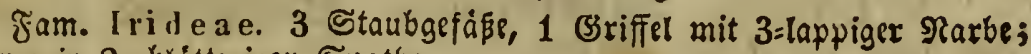
Blume in 2 =blátteriger Epatba.

(Satt. Iris 124, Giadiolus, Crocus.

Zam. A maryllidea e 6 Staubgefäße, ઉriffel mit 3 = Enotiger Narbe; Blume in $1=$ blátteriger Epatha.

Satt. Galanthus, Leucoiun, Narcissus; Amaryllis.

C. Hypogyneae. Dis 4-6= Staubaefábe find alle frudibar und fián an ben gerigonbláttern; $\mathscr{F} r u d t$. enoten oberfíadig.

Zam. Li liaceàe. Frucht eine $3=$ Elappige, vielfamige Rapfer; Eamen mit weider, fdrwammiger Ieffa; Etine Spatha; 3roiebels getrádbie.

હatt. Fritillaria, Lilium 131, Tulipa 131.

Fam. A sph od el a e. Frudte eine runblidye Fapfer ober Beere, $3=$ fádjerig; bie Gamen flein mit harter, fábrarzer Ieffa.

a. 3̦tiebelgerwáchfe ohne Stamm.

હatt. Allium 131, Ornithogalum. Hyacinthus 131.

b. Feine 3rniebel, aber ein Stamm.

Batt. Aloë, Asparagus 131.

Fam. S mil a c a e. \$perigon glockerfobrmig, niditt tief getheilt, biss

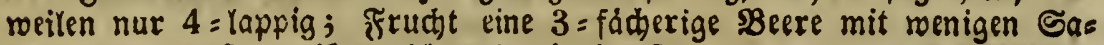
men, beren Zefta weí, wovich und hautig if.

ßsatt. Majanthemum, Convallaria, Polygonatum, Ruscus, Smilax.

Fam. Ml e la nthi a ceae. Frutht befteht at: 3 mit der Raht ans einanber grtoadjenen Batgeapfern; Samen zablreich, mit bäutiger Ieffa.

Batt. Colchicum 132, Veratrum.

Fam. J u n ca gi i ne a e (ङatt. Scheuzeria, Triglochin).

8. Dronung. Tripetaloideae. Nur bie 3 ober 4 \$e: rigonblätter bes inneren Sireifes gefårbt, aud gróßer uno mebr fronenartig; bie bes àußeren Fireifes fino grin.

A. Catogyneae. Frudtenoten unterftanoig. Fam. Broineliaceae, Hydrocharideae.

B. Hypselogyneae. Frudtenoten oberifandig. Fam. P a ride a e. Spcrigon $6-8=$ blátterig; frudtet eine 3-4 fád̆erige Beere; Samen mit f́broarzer Iefta.

Gatt. Paris 133.

Fam: B nt to meae. Sperigon 6 = brátterig, 9 ober melgr Staubges

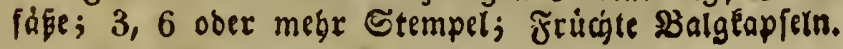

(Batt. Butomus. ఠ. 134. 
Finfte Sinfle. Bracteatae.

Das Sherigonum ift gruin ober wenigftens nidyt fdyon ge: färbt; es befteit of nod) nus 6 , báufig nur nus 3,2 oder 1 SBlatt, Doer fehlt ganz, und sie SBraftea vertritt feine Stelle. Fruchttenoten oberftänoig.

9. Sronung. Spadicineae: SBluthenftano ein einfa= d)er ober veráftelter Folber.

f̧am. Palmae S. 155.

Fam. Aroideac, Typhoideae, Potamophilae.

Zam. Lemnaceae S. 124.

10. Sronung. Glumaceae. Bhithenftand eine 2fefre oder Sisłe.

A. Mit mehrjamigen frúctern. Ple ospe rmae.

gram. Juncea e, Restiaceae.

B. Mit einfamigen rrübten, Mo nosperma e

Zam. C y peraceac.

Fram. Gramineae. E. 124 .

Dabin audi Oryza S. 131.

\section{Acotyledoneae.}

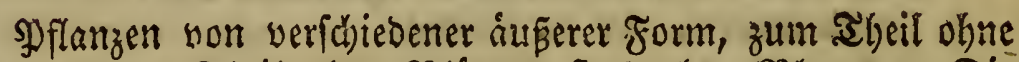
Strmm, jum İheil obne SBlátter, ftets ofhe B̉lumen. Die Samen haben Eeineit Seim, alio audh Eeine Samenlappen, fonbern beftebeat bloz aus einem SBlízchen.

Sechste Slaffe. Cryptogamicae. (Siebe Seite 157)

11. Sronung. Filices.' S. 158.

12. Sronung. Musci.

Fram. Musci frondosi $\mathfrak{S} 159$.

Fam. Musci hepatici S. 159.

13. Sronung. Lichenes. S. 159.

14. Soronung. Algae. S. 160.

15. Sronung. Mycetes. S. 160.

\section{Nerbefferungen.}

Ceite 25 3eile 3 v. o. lieg Cricetus ftntt Gricetus

- 75 - 1 v. D. 1. $2-4$ ff. $2-9$

- $98-10$ v. u. i. vielen fi. 4 .

$-152=22$ v. u. l. Coniferae ff. Coniterac

$=159=3$ v. o. $\mathrm{l}$. frondosi ft. trondosi 


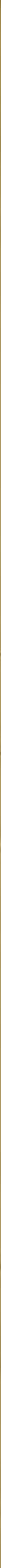


